\title{
HEWLETT-PACKARD जם
}

APRIL 1990

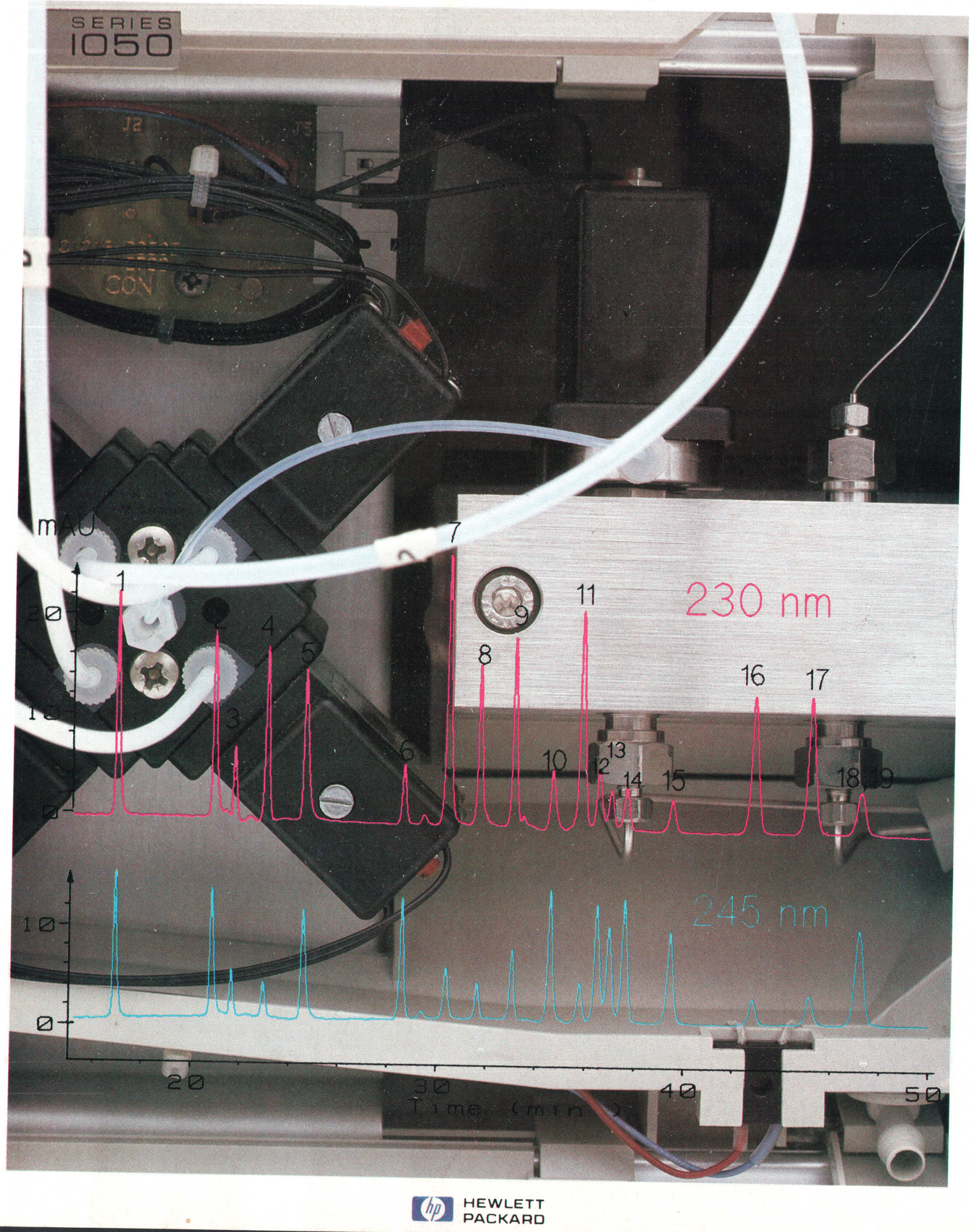




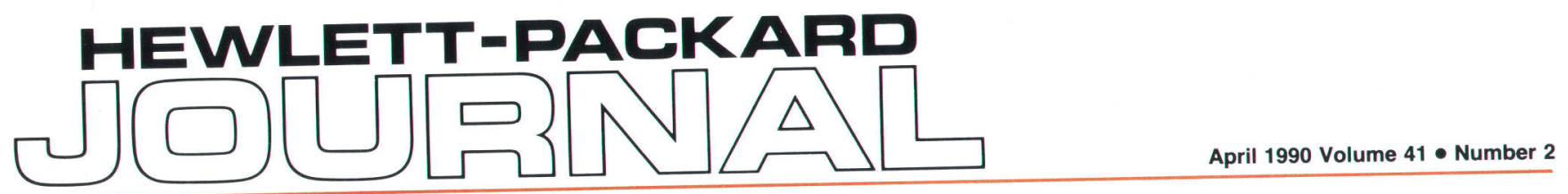

\section{Articles}

8

A New Modular High-Performance Liquid Chromatograph, by Herbert Wiederoder

7 An Introduction to Liquid Chromatography

9 Industrial Design and Ergonomics

Quality Engineering for a Liquid Chromatography System, by Helge Schrenker and Wolfgang Wilde

14

Design for Manufacturing

A Compact, Programmable Sample Injector and Autosampler for Liquid Chromatography, by Wolfgang Kretz and Gerhard Ple

Flexible, Precise Solvent Delivery for Liquid Chromatography, by Fred Strohmeier and Klaus Witt

30 Pump Control Chip

A New Generation of LC Absorbance Detectors, by Axel Wiese, Konrad Teitz, Volker Brombacher, Günter Höschele, and Hubert Kuderer

Firmware Development for a Modular Liquid Chromatography System, by Christian Büttner, Fromut Fritze, and Gerhard Ple

51 HP OpenView Network Management, by Anthony S. Ridolfo 54 HP OpenView Network Management Architecture, by Keith S. Klemba, Mark L. Hoerth,

Editor, Richard P. Dolan • Associate Editor, Charles L. Leath - Assistant Editor, Gene M. Sadoff - Art Director, Photographer, Arvid A. Danielson Support Sol 
HP OpenView Windows: A User Interface for Network Management Solutions, by Catherine J. Smith, Arthur J. Kulakow, and Kathleen L. Gannon

HP OpenView BridgeManager: Network Management for HP LAN Bridges, by Andrew S. Fraley and Tamra I. Perez

HP OpenView Data Line Monitor, by Michael S. Hurst

Network Management for the HP $\mathbf{3 0 0 0}$ Datacom and Terminal Controller, by Serge $Y$. Amar and Michele A. Prieur

85 Developing a Distributed Network Management Application Using HP OpenView Windows, by Atul R. Garg and Lisa M. Cole

\section{Departments}

$\begin{aligned} 4 & \text { In this Issue } \\ 5 & \text { Cover } \\ 5 & \text { What's Ahead } \\ 92 & \text { Authors }\end{aligned}$

The Hewlett-Packard Journal is published bimonthly by the Hewlett-Packard Company to recognize technical contributions made by Hewlett-Packard (HP) personnel. While the information found in this publication is believed to be accurate, the Hewlett-Packard Company makes no warranties, express or implied, as to the accuracy or reliability of such information. The Hewlett-Packard Company disclaims all warranties of merchantability and fitness for a particular purpose and all obligations and liabilities for damages, including but not limited to indirect, special, or consequential damages, attorney's and expert's fees, and court costs, arising out of or in connection with this publication.

Subscriptions: The Hewlett-Packard Journal is distributed free of charge to HP research, design, and manufacturing engineering personnel, as well as to qualified non-HP individuals, libraries, and educational institutions. Please address subscription or change of address requests on printed letterhead (or include a business card) to the HP address on the back cover that is closest to you. When submitting a change of address, please include your zip or postal code and a copy of your old label.

Submissions: Although articles in the Hewlett-Packard Journal are primarily authored by HP employees, articles from non-HP authors dealing with HP-related research or solutions to technical problems made possible by using HP equipment are also considered for publication. Please contact the Editor before submitting such articles. Also, the Hewlett-Packard Journal encourages technical discussions of the topics presented in recent articles and may publish letters expected to be of interest to readers. Letters should be brief, and are subject to editing by HP.

Copyright (c) 1990 Hewlett-Packard Company. All rights reserved. Permission to copy without fee all or part of this publication is hereby granted provided that 1) the copies are not made, used, displayed, or distributed for commercial advantage; 2) the Hewlett-Packard Company copyright notice and the title of the publication and date appear on the copies; and 3) a notice stating that the copying is by permission of the Hewlett-Packard Company appears on the copies. Otherwise, no portion of this publication may be produced or transmitted in any form or by any means, electronic or mechanical, including photocopying, recording, or by any information storage retrieval system without written permission of the Hewlett-Packard Company.

Please address inquiries, submissions, and requests to: Editor, Hewlett-Packard Journal, 3200 Hillview Avenue, Palo Alto, CA 94304, U.S.A 


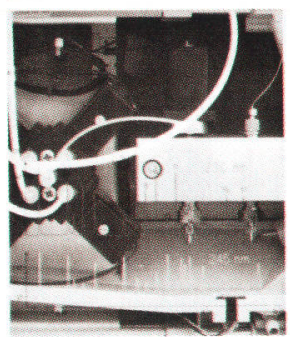

To determine the composition of a complex mixture such as a biological sample or seepage from a toxic waste dump, chemists often use a combination of gas or liquid chromatography and mass spectrometry. The chromatograph separates the compounds that make up the mixture (see page 7 for an explanation) and the mass spectrometer gives information on the masses and structures of the constituent molecules. For many samples, especially those that are too nonvolatile or thermally unstable to be changed to a gas without decomposing, liquid chromatography is the best method of separaSeries LC modules, described on pages 6 to 50 of this issue. As R\&D section manager Herbert Wiederoder explains in his introduction on page 6, the design of a high-performance LC system requires contributions from materials science, mechanics, chemical physics, optics, electronics, and software. The problems include the handling of liquids having a wide range of solvent properties at widely varying flow rates and pressures, control of mechanical components such as pumps, motors, and valves, detection of chemical substances, and data handling. The HP 1050 Series extends and refines HP's LC technology, emphasizing a common architecture and a standard design for all modules. A clever feature of the cabinet design is a built-in leak drainage system. When the modules are stacked, leaks drain from higher to lower modules without special adapters. There are three types of HP 1050 modules: solvent delivery system (pump module), autosampler, and detector. The pump (page 24) is a simple and reliable series-dual-piston design with compensation for liquid properties and major physical side effects built into the pump control system. The autosampler (page 17) can automatically inject samples in a programmed sequence selecting from either the standard 21-sample tray or the optional 100-sample tray. Two types of HP 1050 detector modules are available (page 36). Both are absorbance detectors, which measure the light absorbed by the sample as a function of wavelength, producing a spectrum that is characteristic for a particular substance. The forward optics detector passes a single wavelength of light at a time through the sample, while the reverse optics detector uses white light, breaks the light into separate wavelengths after it goes through the sample, and detects all wavelengths at once using a photodiode array. The HP 1050 Series firmware design is described in the article on page 44. In keeping with the standard-design philosophy, about $60 \%$ of each module's firmware is the same in all modules. To ensure that the HP 1050 Series modules met their quality objectives, a formal program was designed; it's discussed in the article on page 11.

In the 1980s, computer technology became pervasive in companies large and small. The 1990s will be the decade of the network, as companies interconnect their mainframe computers, personal computers, engineering workstations, and manufacturing systems. Already, complex networks of computers and data communications equipment are common, usually containing equipment from many different manufacturers. These networks challenge their managers to find ways of monitoring and troubleshooting them, controlling the individual parts, measuring performance, and accounting for the use of network resources. The HP OpenView family is a set of hardware and software products designed to address these issues in the management of open, standards-based, multivendor networks. "Standards-based" is a key concept, for it is the networking standards being 
developed by the computer industry and international organizations like the ISO that make multivendor networks both possible and manageable. The HP OpenView family currently includes HP OpenView Windows (see page 60), which provides a consistent, friendly user interface and an integrated environment for managing networks, the HP OpenView BridgeManager (page 66) for managing LAN bridges, the HP OpenView Data Line Monitor (page 71) for monitoring analog data lines, and the HP OpenView DTC manager (page 76) for configuring, diagnosing, and controlling datacom and terminal controllers. The HP OpenView story begins with an overview on page 51. The HP OpenView network management architecture, solidly based on international and de facto industry standards, is discussed in the article on page 54 . The article on page 85 describes a pair of network management tools that were developed to test and refine the HP OpenView concept. These tools are used internally at HP but aren't available as products.

\section{A Word about Cards}

With the December 1989 issue, we sent a card to our U.S. readers, asking them to confirm that they wanted to be on our mailing list. Because a last-minute change of plans wasn't properly communicated to our U.S. printer, most readers received the wrong card, which implied that they had recently become subscribers. We'd like to apologize to the hundreds of long-time readers who were offended or confused by this error. We'd also like to thank the many readers who returned their cards with kind comments.

R.P. Dolan

Editor

\section{Cover}

Behind the front door of the HP 1050 Series liquid chromatograph quaternary pump module is the four-way proportioning valve and the dual-piston pump. The overlay is a pair of chromatograms made using an absorbance detector at two different wavelengths.

\section{What's Ahead}

In our next issue, we'll have articles on the design and capabilities of the HP SoftBench integrated software development environment, on the HP OSF/Motif window manager and the HP OSF/Motif widget library, on the HP particle beam interface for combining liquid chromatography with mass spectrometry, and on HP's membrane probe technology for testing integrated circuit wafers. 


\title{
A New Modular High-Performance Liquid Chromatograph
}

\author{
The HP 1050 Series of modules refines and extends HP's \\ LC technology, emphasizing a common architecture and \\ a standard design for all modules.
}

\author{
by Herbert Wiederoder
}

\section{IGH-PERFORMANCE LIQUID CHROMATOGRA-}

PHY (HPLC), as a separation technique for nonvolatile substances in mixtures, has a wide range of applications in the industrial world today. Over the last decade, instrumentation for liquid chromatography has evolved towards higher flexibility, greater ease of use, and higher reliability.

Designing an HPLC system requires a high degree of interaction between various disciplines, including mechanics, materials science, chemical physics, optics, electronics, firmware, and software. Problems that must be solved include the handling of liquids having a wide range of solvent properties at flow rates from $1 \mu \mathrm{l} / \mathrm{min}$ to $10,000 \mu \mathrm{l} / \mathrm{min}$ and pressures up to 400 bar, control of mechanical components, detection of chemical substances, and data handling.

How these problems were solved to provide a flexible, easy to use, easy to handle, and highly reliable HPLC system for HP's latest-generation liquid chromatograph will be described in this and the following articles. The design of the new system, called the HP 1050 Series Liquid Chromatography Modules, takes a new modular approach, starting from the technology of the HP 1090 LC family. ${ }^{1}$

\section{HP 1050 Architecture}

An HP 1050 Series module is an independent instrument with specific user, hydraulic, and communication interfaces. Each module has its own power supply and enclosure. The combination of the modules provides the functions necessary to do HPLC. The information flow and solvent flow in a typical chromatograph are shown in Fig. 1.

The HP 1050 architecture breaks down the modules according to the functional blocks shown in Fig. 1. Fig. 2 shows the HP 1050 Series modules. The separation takes place in the column, which is a tube filled with porous material. The typical column supported by the HP 1050 has an internal diameter of $4.6 \mathrm{~mm}$ and a length between 100 and $250 \mathrm{~mm}$. The column is placed in the solvent preparation module, which is part of the solvent delivery system. Two versions of solvent delivery modules are available: an isocratic pump version and a quaternary pump version. If the quaternary pump is chosen, the solvent preparation option is required. It is typically placed above the pump and serves several functions, as described in the article on page 24 .

The sample (sample volume typically between 0.1 and $100 \mu \mathrm{l}$ ) is introduced either manually by a valve or automatically by a separate module called the automatic liquid sampler (ALS). Samples are typically stored in 2-ml vials. Up to 119 vials can be automatically processed, as described in the article on page 17.

The detector is also a separate module. Two versions of absorbance detectors are available: a variable wavelength detector (VWD) and a multiple wavelength detector (MWD).

\section{Standard Module Design}

Before the individual modules went into the detailed design phase, a common internal and external architecture for all the modules was developed, and standards were established for all modules to follow. Standardization was a crucial issue. It provides several advantages, including:

- Provides a family look for the customer

- Gives the customer the freedom to arrange the modules to optimize the use of bench space

- Lowers the cost of the individual modules

- Allows new production processes for medium-volume products

- Increases R\&D productivity and efficiency

- Makes design for manufacturing easier

- Defines clear interfaces between the functional modules for a liquid chromatograph.

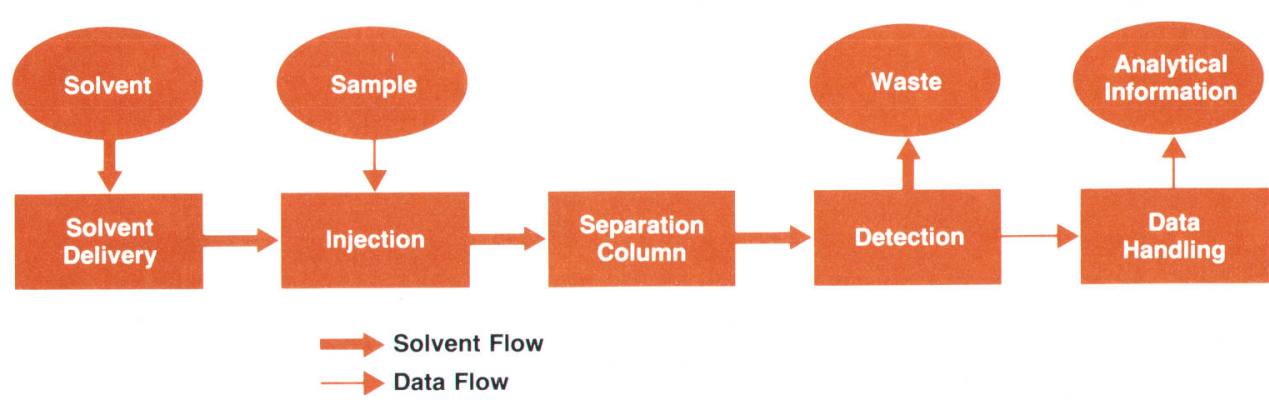

Fig. 1. Functional diagram for high-performance liquid chromatography (HPLC). 


\section{An Introduction to Liquid Chromatography}

At the turn of the century the Russian botanist Tsvett was investigating the composition of the dyes in green plant leaves. He made petroleum ether extracts of freshly dried leaves and injected a small amount of such an extract on top of powdered calcium carbonate, which was contained in a vertically placed glass tube or column. When he flushed the column with a mixture of petroleum ether and alcohol, the dyes moved down through the column with different velocities and finally formed a series of green and yellow zones (see Fig. 1). He let the column run dry and pushed the calcium carbonate out of it. He divided this into pieces containing the different zones, and dissolved the dyes (which are green chlorophyls and yellow carotenoids) from the adsorbent (the calcium carbonate) with an appropriate solvent.

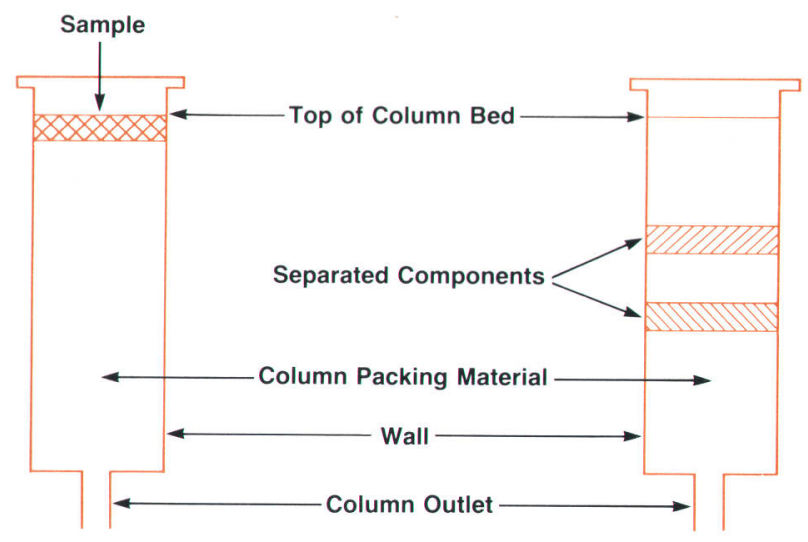

Start: Sample Introduced at Top of Column

End: Components Separated

Fig. 1 Principle of chromatography.

In this way Tsvett physically retrieved the individual components from a mixture. He called this separation technique chro- matography, not because "tsvett" is Russian for color (Greek "chromos" = color), and not because the zones in his first experiments were visible as colored rings (he immediately recognized that the technique is suitable for both colored and uncolored compounds), but because the mixture of dyes was separated obeying some physical law, like the colors in a rainbow. He drew this parallel because the dyes were always separated in the same order when he used the same composition of the flushing solvent.

In all chromatographic techniques two phases are involved: (1) a stationary phase, which is the column packing material, the calcium carbonate in Tsvett's experiments, and (2) a mobile phase, the eluent, which is the liquid that is flushed through the column bed, the petroleum ether/alcohol mixture in Tsvett's experiments. The components in the sample mixture are separated because their interaction with the stationary phase is different. Because this interaction mechanism can be influenced in many ways, both by changing the stationary phase material and by changing the mobile phase composition, chromatography has become one of the most powerful and most important chemical analysis techniques.

In the last decades, liquid chromatographic techniques have improved dramatically. It is now very unusual for the column packing material to be removed after each experiment. Instead, the column is flushed until all compounds of the sample mixture have left the column outlet. The stationary phase can then be used again; this is necessary for routine analysis.

The compounds that leave the column outlet are now usually detected on-line. Many powerful detection techniques have been developed, of which the UV/visible absorption detector is the most widely used. Other detector types are fluorescence, electrochemical, radiometric, conductance, refractive index, polarimetric, and mass spectrometric. This wide choice of detectors includes types that offer universal detection (e.g., refractive index) or types that detect very specific functional groups with increased sensitivity (e.g., fluorescence). This is helpful in identifying compounds.

(continued on next page)

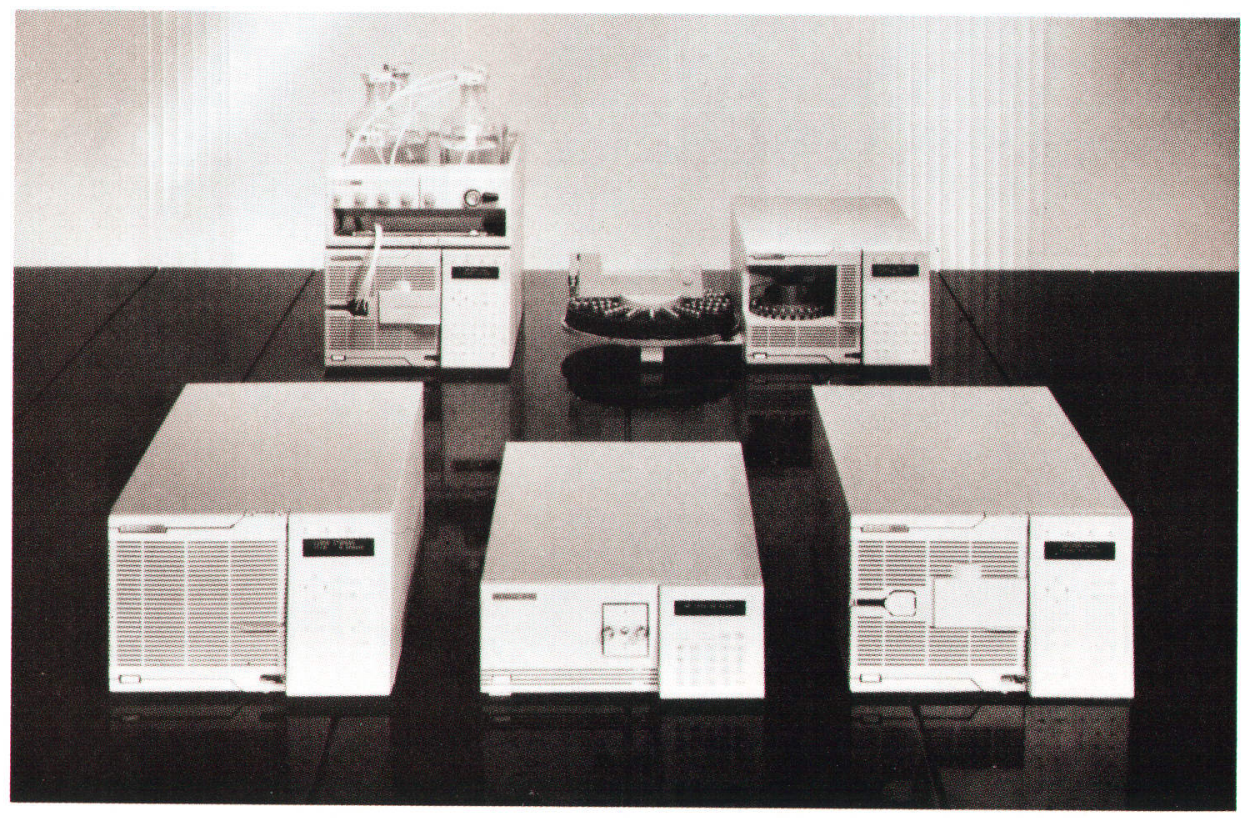

Fig. 2. The HP 1050 Series Liquid Chromatography Modules. 
The gravitational force Tsvett was using to elute the compounds for his separation does not always give very reproducible results. Deviations in column resistivity (caused, for example, by changes in the column bed or by temperature effects) lead to changes in the flow rate of the eluent. This means that the same compound may elute from the column at varying times. Modern pumping systems can supply liquids with hardly any pulsation and with very high accuracy, enabling compounds to elute from the column at very reproducible times (relative standard deviation $<0.5 \%$ ). This retention time is one of the most important parameters in the identification of compounds. Even more flexibility (and complexity) in the separation process can be achieved by changing the solvent composition during the analysis. For this purpose highly sophisticated programmable-gradient pumps are available.

Once the hydrodynamics of the separation were understood and written down in some fundamental equations, it was clear that the proper choice of column packing material can improve the separation considerably. The smaller the diameter of the packing material particles, the better the separation efficiency. The practical limit is near 1 to $2 \mu \mathrm{m}$, with the most common particle diameter nowadays being $5 \mu \mathrm{m}$.

The particle diameter should be constant within narrow tolerances: the more uniform, the better. With these small particle diameters, with column inner diameters of 2 to $5 \mathrm{~mm}$, with common lengths of 10 to $25 \mathrm{~cm}$, and with eluent flow rates of 0.2 to $2.5 \mathrm{ml} / \mathrm{min}$, the pressure drop can be several hundred bar. Therefore, glass is not suitable anymore for the column tube material. Now, tubes of stainless steel are common. As a consequence the pumping systems have to be able to deliver pressures up to approximately 400 bar. In addition, the particles must have

As a result of standardization in the hardware and firmware, the number of parts has been significantly reduced compared to the HP 1090. The HP 1050 has about $60 \%$ fewer part numbers than the HP 1090. About $60 \%$ of the firmware code in each module is the same in all modules.

The external design for all the modules conforms to HP corporate product guidelines. All modules have the same footprint (325 mm wide by $560 \mathrm{~mm}$ deep) and user interface style.

Ease of access to electrical and hydraulic interfaces was an important design goal. This resulted in a design in which all electrical connections are made from the rear and all hydraulic connections are made from the front. Because the instruments are in contact with a wide range of solvent properties, for safety reasons a special leakage interface was developed to handle the solvent in case something leaks inside a module.

The internal architecture was driven by several design goals, including design for manufacturability, standard internal structure, easy accessibility to routine maintenance parts, and user interaction from the front.

Design for manufacturability was an important issue for the enclosure. The enclosure is based on a chassis that holds all the mechanical, electrical, and cooling assemblies and the front panel. The cover, fixed with two screws, completes the enclosure.

Fig. 3 shows the internal structure of all of the modules. The back part contains up to six standard-sized printed circuit boards including a standard power supply. All of the boards have their own rear panels and are fixed in a high mechanical strength. Therefore, most column packing materials have a silica gel core. This silica gel is usually chemically modified and stabilized by attaching less-polar groups to it. This makes the separations from column to column more reproducible, and makes fine tuning of the stationary phase to different separation systems possible.

All chromatographic techniques use the fact that the detector signal is proportional to (preferably linear with) the concentration of the compound that is being detected. This means that the amount injected onto the column must be supplied with very high precision and reproducibility. Either fixed-volume (manual) injectors are used, or variable-volume (automatic) injectors. In the latter, the injection system is combined with a sample exchanger, which allows automatic analysis of a large number of samples.

Some samples are not thermally stable. For such samples it may be necessary to cool the sample to approximately $4^{\circ} \mathrm{C}$. Cooled autosamplers are now available.

The separation kinetics usually are temperature sensitive. Therefore, thermostatic control of the column temperature is necessary for the highest reproducibility of the retention times and the peak shapes.

All of these contributions have made liquid chromatography a very powerful and reproducible technique, so much so that the modern version is often referred to as high-performance liquid chromatography, or HPLC

Henry J. van Nieuwkerk

Chemist

Waldbronn Division

flexible card cage. The card cage isolates the electronic section from the mechanical and cooling section and makes the cable connections to the mechanical parts. The mechanical sections are arranged to provide access to the routine maintenance parts from the front. The cooling system is designed to channel the air flow from the front to the back. This makes it possible to stack the modules without sac-

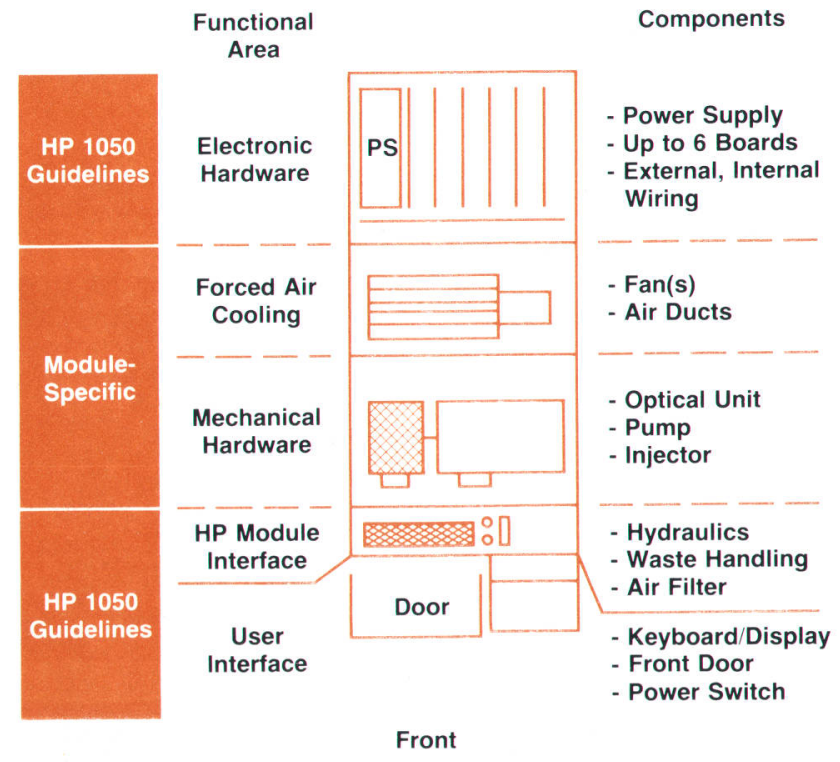

Fig. 3. Internal module structure. 


\section{Industrial Design and Ergonomics}

"Those who want to be successful in international markets need well-designed products. However, design is more than just that, it is part of the product's quality, it communicates the company's basic values, and therefore it may be an important criterion in the purchasing decision for high-tech products."1 One effect of changing social values is that users are increasingly more selective in their demands on the human interfaces of products. They require much more than just good styling. The challenge for the industrial design of the HP 1050 Series Liquid Chromatography System was to keep pace with this trend.

Customer visits played a major part in the industrial design investigation. By observing and interviewing users, we were able to determine our own instruments' weak points as well as those of competitive machines: the instruments were loud, maintenance was complicated, access to the inside of the instrument was difficult, the instrument required large amounts of bench space, compatibility with other instruments was low, the user interface was easily misunderstood. Combining this list of customer needs with the technical requirements of other engineers in the team, the industrial designers were able to develop several alternatives for the user interface.

We chose the HP guidelines for computer products as the basis for the industrial design of the HP 1050. This makes the HP 1050 stackable like HP desktop computer products. Stackability, however, required that the internal components be reorganized, since usually instruments of this sort are repaired or modified from the top, by removing the top of the case. The solution was to split the internal components into two separate areas: the electronics in the back in a card cage, accessible from the rear, and the mechanical components at the front, accessible by opening the front door.

To design a simple and common user interface, we aimed to standardize as much as possible, which of course required considerable effort in coordinating our activities. A cardboard model showed external dimensions and aided in estimating the amount of space required on the lab bench.

The realization of the concept had to meet not just the functional demands of the instruments. Many improvements to the front panel had to be made, since they were not covered by the guidelines. A few examples are given here.

The front panel consists of seven plastic moldings, six of which are common to all modules. The door is the only piece that differs from module to module. The keyboard is the only piece fixed by screws - two in all. All the others snap together. The door can be swung open to obtain access to the parts requiring maintenance or modification during operation. The keyboard assemblies for all the 205-mm-tall modules are differentiated by individual silkscreens bearing the module-specific key functions only. The base of the keyboard is intended to act as a handle during transport and as a guide for the capillaries - the liquid connections from one module to the next. A similar treatment in the metal work at the rear means that the instrument can be lifted by one person, unaided.

A difficult problem was posed by leaks that may occur in any liquid chromatograph. Most instruments leave the solution of this problem to the customer. The HP 1050, on the other hand, offers a contribution to the safety and the organization of the instrument by enabling the operator to install capillaries in gutter-like channels, which collect any leaks and direct them to a waste pipe. The drainage system (Fig. 1) consists of a leak basin, a leak sensor, and vertical channels running inside the front panel of each module. A channel in one module directs liquid to the

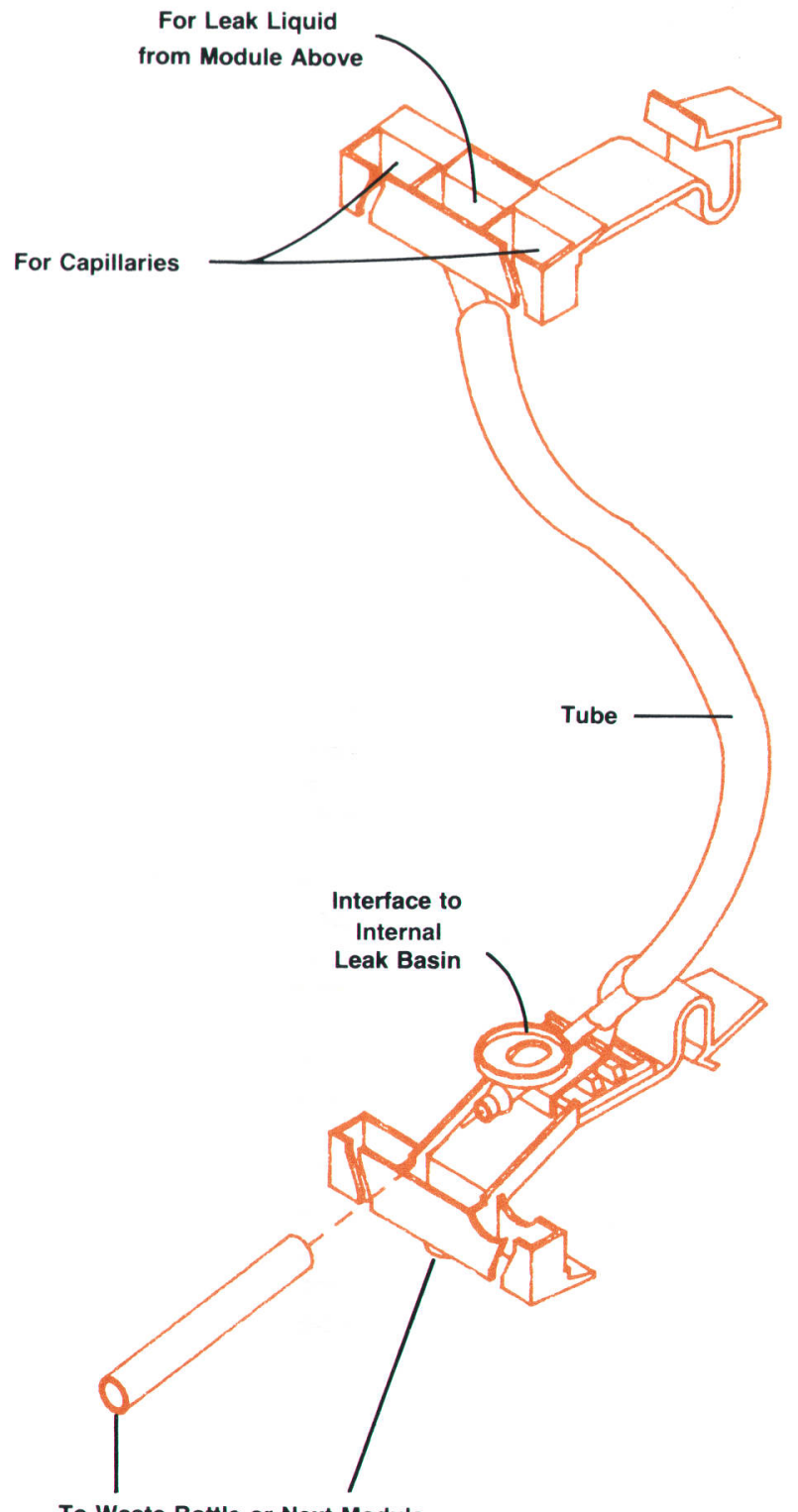

To Waste Bottle or Next Module

Fig. 1. Leak drainage system.

channel in the next lower module without the need for special adaptors, so that only the lowest module in the stack needs to be connected to an external waste pipe. Any of the modules can be placed at the bottom of the stack. These leak interfaces are easy to remove for cleaning, which may need to be done more frequently when using liquids with high concentrations of salts, which may crystallize out.

\section{Ergonomics}

A user interface can only be considered well-designed when it can be operated without error, quickly, and without personal danger. Two examples are worthy of mention with respect to the HP 1050. The opening in the side of the autoinjector module allows the injector to be loaded via robotics, for example the autosampler, but primarily this opening is for loading the auto- 


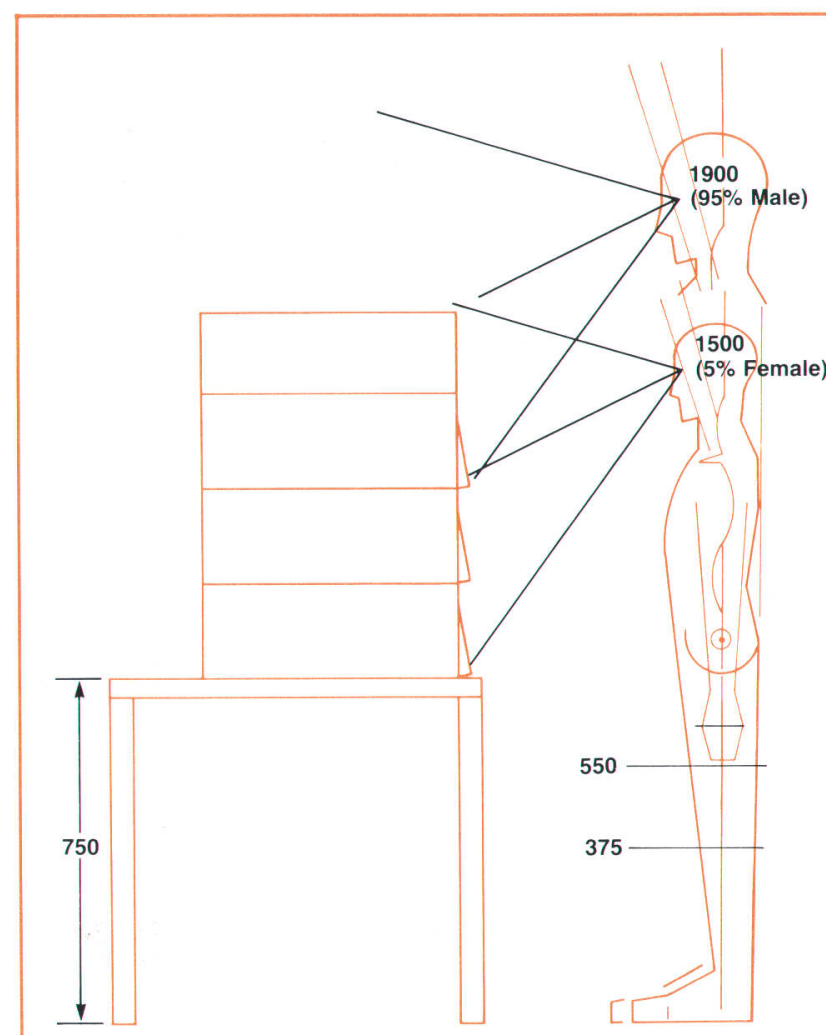

Fig. 2. Viewing angles for the keyboard. Heights are in millimeters above the floor.

injector with the sample tray by hand. The tray is designed such that it always fits right the first time. It is keyed such that there is automatically only one way to lower it onto the spindle-in the zero starting point for subsequent rotation during sampling. This avoids incorrect mounting and prevents injuries. The wide opening in the autosampler and the tray's grip-fast handle avoid handling errors such as slippage or impacts while placing the tray in such a confined space.
The second example is the keyboard. We had to find the best compromise among several parameters. Every module can be installed at the top or at the bottom of the stack, can be installed on a high or a low bench, and might be operated by a tall (95\%) or a short $(5 \%)$ person, both of whom should be able to read the display. Different mounting angles were simulated in several tests of the keyboard and display (Fig. 2), as were different types of displays. The best combination was found to be a vertical vacuum fluorescence display with a blue/green filter and a touchsensitive membrane switch keyboard inclined at 10 degrees from the vertical. We decided to avoid using grey areas around the keys since we wished to give the impression that the keyboard would be simple to use (there are 35 keys in the confined space of each keyboard) and to reduce the likelihood of erroneous input.

\section{Conclusion}

The HP 1050 LC system requires little bench space since it stacks easily. All important parts are easily accessible and the user interface is easily understood, comfortable to use yet functional. The automation interface and case concept are as futureproof as we could make them, and last, but not least, the product has an attractive, up-to-date appearance. We are pleased that the HP 1050 has been awarded international design prizes.

\section{Acknowledgments}

Industrial design is only as good as its implementation. I would like to thank all managers for their support of our efforts in reaching this goal and the mechanical engineers, especially Werner Schneider who worked on the common parts of the front panel, Hans-Peter Zimmermann who worked on details in the autoinjector, Hans-Georg Haertl who designed the solvent cabinet and whose ideas formed the basis of the leak interface, and Bernhard Dehmer who made a major contribution to the concept.

\section{Reference}

1. M. Deppisch, "ENORM in FORM," Elektronik Praxis, June 1989, p. 44

Raoul Dinter

Industrial Designer

Waldbronn Analytical Division rificing the cooling. The front part of the module contains the user interface, a door for access to the mechanical parts, the hydraulic interfaces for the capillaries, and the leakage interface.

The technical details of how the individual modules use the standard components and are designed for their specific requirements are covered in the other HP 1050 articles in this issue.

\section{Acknowledgments}

The HP 1050 evolved from technologies developed for the HP 1090, which was introduced in 1983. Contributions to this complex and multidisciplinary family of instruments came from many people in our division. A great deal of support came from HP Waldbronn Division management, notably Alfred Maute, Hans-Günter Hohmann, Günter Nill, Dieter Lindenau, and Helge Schrenker. The joint effort between marketing, manufacturing, QA, and R\&D made the project fun. I would like to give special credit to the individual project leaders: Fred Strohmeier (pump), Axel Wiese (multiple wavelength detector), Gerhard Ple (automatic liquid sampler and firmware), and Konrad Teitz (variable wavelength detector).

\section{Reference}

1. Hewlett-Packard Journal, Vol. 35, no. 4, April 1984, entire issue. 


\title{
Quality Engineering for a Liquid Chromatography System
}

\author{
For the HP 1050 Series LC system, customer expectations \\ were translated into measurable quality goals, which were \\ then verified by special test methods.
}

by Helge Schrenker and Wolfgang Wilde

USTOMER SATISFACTION is the ultimate benchmark for product quality. This sounds straightforward, but it is far from a trivial task to translate the customer's voice into product quality. It requires finding out, in quantitative and measurable terms, what quality properties the majority of potential customers want, translating these customer expectations into terms, measures, and goals that are meaningful to design and manufacturing engineers, and assuring throughout the design and transfer phases that these goals will be met by the future product.

For two product generations, we have been using the same set of quality criteria. With each generation, we have refined the measures and test methods. Fig. 1 shows the key quality criteria and measures that were applied to the HP 1050 Series Liquid Chromatography System. Using some of these criteria and measures as examples, we will briefly describe how specific quality goals for the HP 1050 Series were set and verified through specially developed test methods.

\section{Setting Quality Goals}

Design goals for each of the five quality criteria listed in Fig. 1 were set using three information sources:

- A specific, well-aimed customer survey

- Inputs from senior sales and service people on their perceptions of customer expectations

\begin{tabular}{ll} 
Quality Criteria & \multicolumn{1}{c}{ Measures } \\
Performance & $\begin{array}{l}\text { Conformance to Published Specifications } \\
\text { Result Precision and Confidence } \\
\text { Consistency over Longer Operating } \\
\text { Periods }\end{array}$ \\
Reliability & $\begin{array}{l}\text { Mean Time between Failures (MTBF) } \\
\text { Useful Life of Product }\end{array}$ \\
User Friendliness & $\begin{array}{l}\text { Time for Standard Tasks, } \\
\text { Unfamiliar/Familiar Operator } \\
\text { System Installation Time }\end{array}$ \\
& $\begin{array}{l}\text { Number of Cables and Capilliary } \\
\text { Connections } \\
\text { Noise Emissions }\end{array}$ \\
Serviceability & $\begin{array}{l}\text { Repair and Maintenance Cost to the User } \\
\text { Average Downtime per Year } \\
\text { Mean Time to Repair }\end{array}$ \\
Regulations/Safety & Conformance to Relevant Standards
\end{tabular}

Fig. 1. Key quality criteria and measures applied to define and verify the quality of the HP 1050 Series LC system.
- Analysis of presently available LC instrumentation (strengths, weaknesses, potential for improvements).

The customer survey was aimed at gathering current customer expectation data on such sensitive criteria as reliability, uptime, measurement reproducibility, cost for service and maintenance, and so on. Since our resources were limited, we decided to survey only a selected, representative sample of about 100 individuals from our customer base. Our experiences with such small samples in earlier surveys were good. The advantages are low cost and the possibility of enhancing the return rate and results by a mix of mail, telephone, and personal survey methods.

We achieved excellent consistency of survey results. The responses, even to sensitive questions, were very consistent, and compared well with the perception of senior HP field and marketing people and with the results of a thorough technical analysis of a current similar product. (The technical analysis was an analysis of the failure modes of a current product assuming a complete redesign with elimination of all known failure mechanisms for which solutions seemed feasible.)

It was interesting to learn how dramatically customer expectations for the useful life of the product can change from generation to generation. Expectations for product

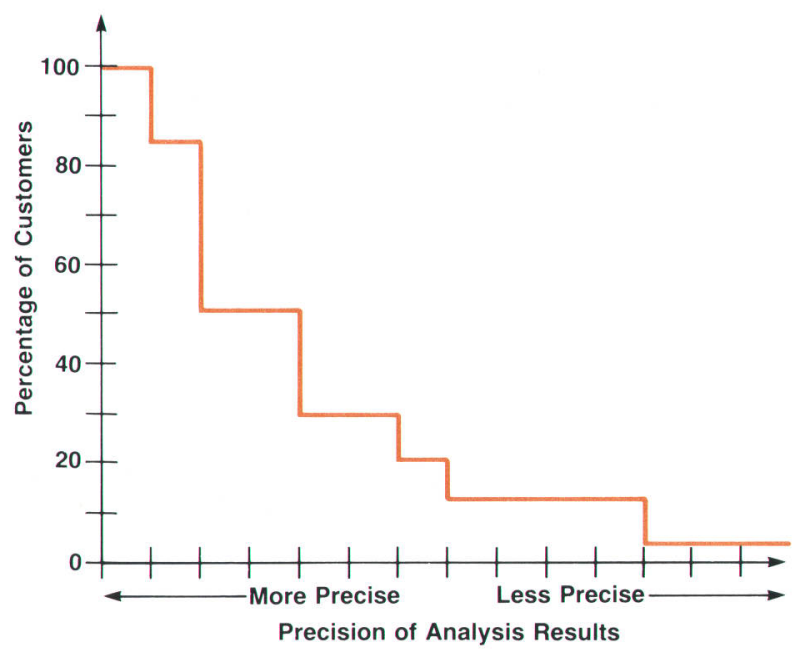

Fig. 2. Results of the customer survey on expected analysis precision, showing the cumulative percentage of customers whose applications are such that they could accept precision equal to or better than a particular level. 
lifetime were more than three times as long as the equivalent data from a survey we had conducted about nine years ago. Setting the right goal for useful life is critical for mechanical assemblies. Higher-lifetime components are often more expensive, so overdesign must be avoided. On the other hand, early wear-out means dissatisfied customers.

One important aspect of surveys is the statistical evaluation of the response data. In this survey, the responses of customers from different markets and different environments for such criteria as expected reliability, service cost, and the like were consistent enough to justify calculating the mean and confidence interval. This was not so for some other results, for example the expected analysis precision, where the responses differed widely based on the respondents' major applications. This was not surprising, since a QC analysis of a drug may require a precision level of $0.3 \%$, while in an analysis of a biological sample in a complex matrix, which requires several sample preparation steps that are all prone to statistical variation, overall expected analysis precision may be only 5 to $10 \%$. In such a situation it is meaningful to plot a cumulative distribution of the survey results as shown in Fig. 2. This plot indicates what percentage of potential users could accept a product designed for a certain performance level (assuming that users with lower performance requirements would accept the product as long as the price were acceptable). This plot is useful for cost/performance optimization.

Similarly, design goals were defined for each of the quality criteria listed in Fig. 1, resulting in a set of benchmarks for HP 1050 Series quality.

\section{Reliability Verification}

There are two main approaches to strife (stress + life) testing. The first is the test-to-fail philosophy: the stress applied to a product is increased until a failure occurs. The aim of this test type is to find design weaknesses using high stress levels. The second approach is a strife test with fixed stress conditions (constant acceleration factor*) at a more moderate stress level, which most likely finds only failure mechanisms potentially occurring under normal operating conditions. The focus is not only on finding design weaknesses but also on being able to predict reliability (annualized failure rate, or AFR). On the product and sys-

*Acceleration factor is the ratio of the annualized failure rate under stress conditions to the annualized failure rate in normal use.

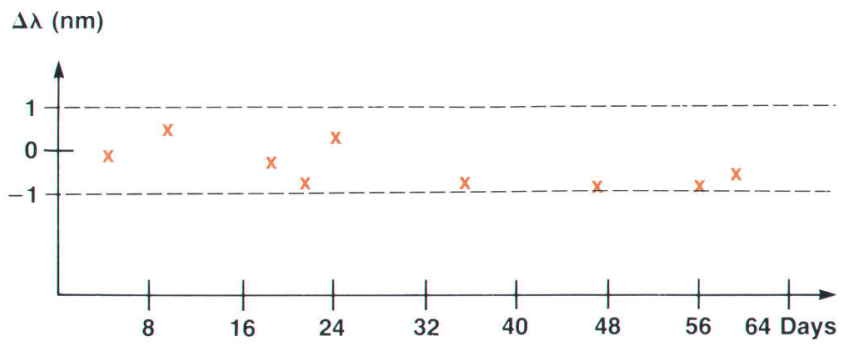

Fig. 3. Plot of the wavelength calibration accuracy of the HP 1050 Series diode array detector. The test time of 64 days corresponds to over two years of normal operation, assuming an acceleration factor of five and 3500 operating hours per year. tem levels, we do mainly the second type of strife testing.

The same product-level stress conditions are also used to check the robustness of specifications against aging. This is possible because we now have more than eight years of experience with this type of test, and we can verify by comparison with field failure results that a typical acceleration factor for a product under our stress conditions lies between 5 and 10. For example, Fig. 3 shows the wavelength calibration accuracy of the HP 1050 Series diode array detector over a simulated operating time of two years. The result is better than $\pm 1 \mathrm{~nm}$, equivalent to the design goal. Fig. 4 is a flow chart of the test procedure. The reason for the additional tests with subassemblies is that, to accelerate a special failure mechanism, specific stress conditions may be necessary and/or more data for failure analysis may be needed.

As mentioned before, we want to be able to predict reliability, that is, reach a good estimate for the annualized failure rate (AFR) during and after completing the test. There are many obstacles to achieving high confidence levels. One has the option of either testing many products in parallel or of applying high stress to a few samples to get results in a reasonably short time. Neither alternative is ideal; it is too expensive to test a large number of complete systems, and very high stress levels risk introducing unrealistic failure mechanisms. Another problem is determining the acceleration factor for a product under high stress. We decided to stay with our moderate stress level and find a good model to monitor reliability growth. We selected the Duane model. It is an advantage of such models

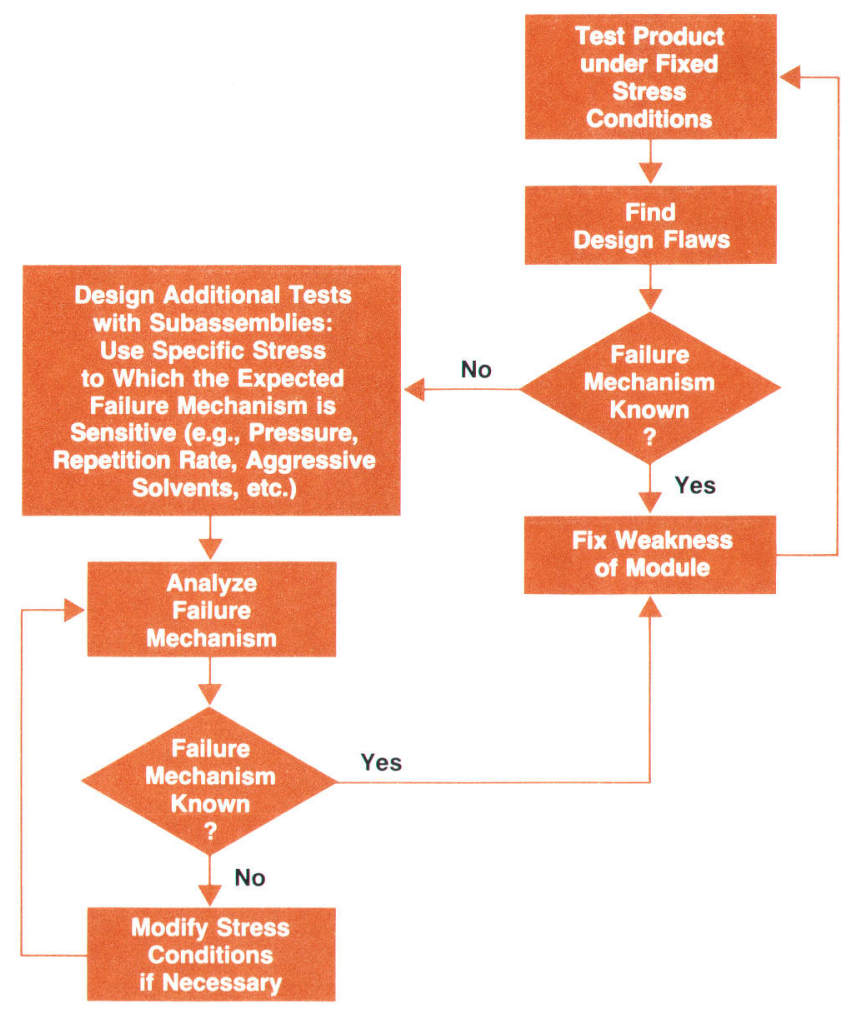

Fig. 4. Test procedure for strife testing under fixed stress conditions. For additional tests with subassemblies, stress conditions are adapted to the failure mechanism. 
that test data for a product under test is accumulated (giving more confident results) to calculate an instantaneous AFR, which successively improves. The model also indicates the effectiveness of the reliability program. ${ }^{1}$

For the HP 1050 Series modules we decided on the following constant stress parameters: temperature cycling between 5 and $70^{\circ} \mathrm{C}$, line voltage and frequency variations, power on/off cycling, and instrument-specific stress for accelerated operation (for example, repetition rates for the injector, pressure for the pump, etc.). For any failure found during the test we did an analysis of the failure mechanism and implemented the fix. The resultant improvement of the reliability of the instrument using this test-fix-test method is called reliability growth. For data evaluation we used the Duane model, which assumes that the cumulative mean time between failures $\left(\mathrm{MTBF}_{\mathrm{C}}\right)$ is proportional to $\mathrm{x}^{\alpha}$, where $\mathrm{x}$ basically is the test time and $\alpha$ is the reliability growth estimator. ${ }^{2}$ Plotting $\mathrm{MTBF}_{\mathrm{C}}$ against $\mathrm{x}$ on log-log paper leads to a straight line with the slope $\alpha$ (see Fig. 5). With these results we were able to predict an AFR at the time of release of the HP 1050 Series. Now, more than a year after the introduction of the HP 1050 Series, we have sufficient data to check this predicted AFR. Analysis shows that we are within $\pm 10 \%$ of the predicted AFR for the HP 1050 pump. These results encourage us to continue using this method.

\section{User Friendliness}

In 1986 we started to develop measures and test methods for user friendliness, to be included later in the design objectives for new product development. The first step was to assemble a group of experienced people from marketing, $\mathrm{R} \& \mathrm{D}$, sales, and service, and brainstorm on factors relevant to the user friendliness of an analytical instrument. The result was a list of about 20 factors that all seemed more or less relevant. To narrow these down to a few testable

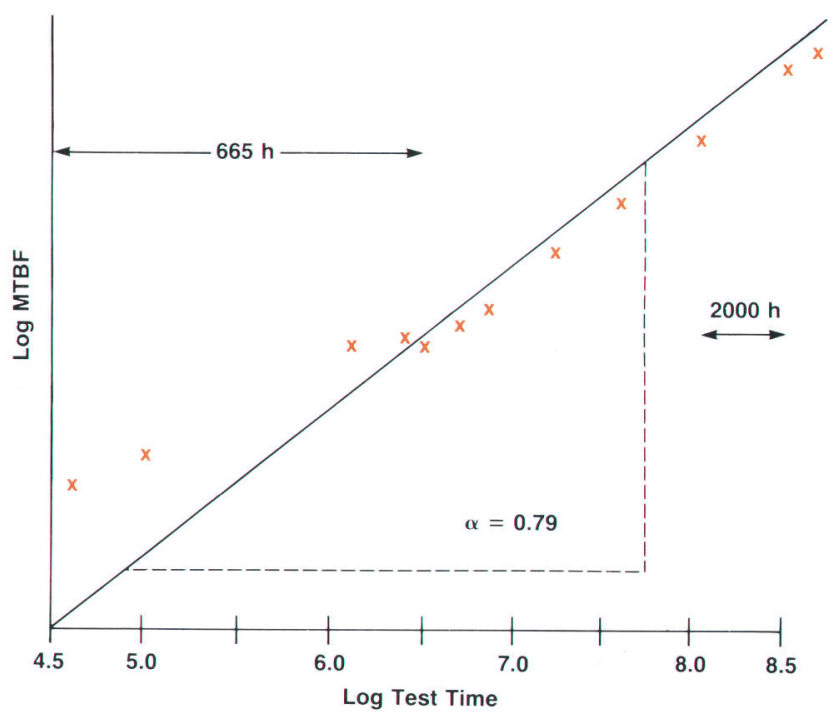

Fig. 5. Duane plot for the HP 1050 Series quaternary pump, relating cumulative mean time between failures $M T B F_{C}$ to test time.

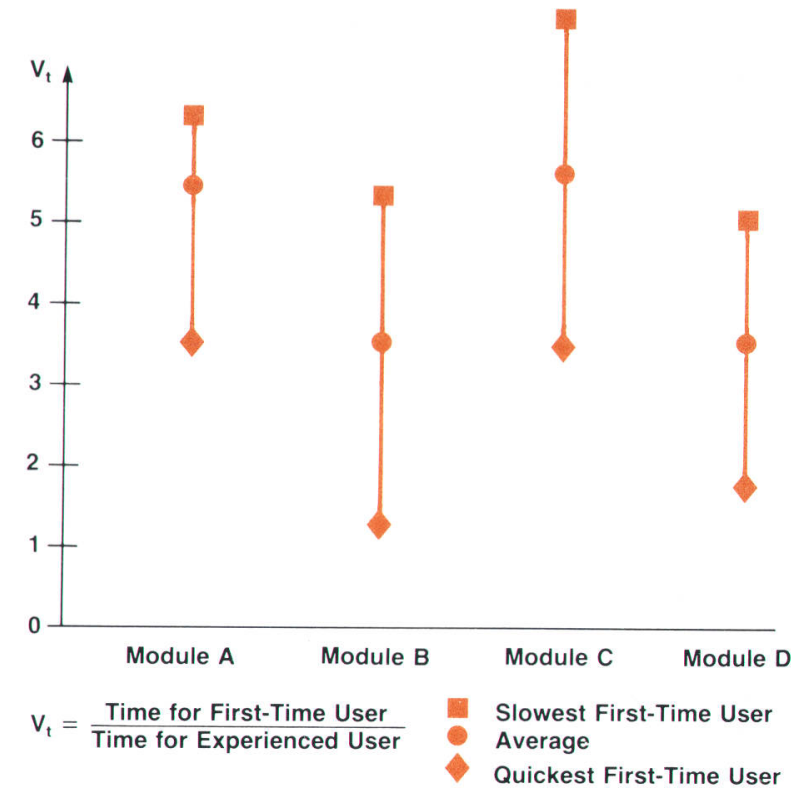

Fig. 6. Results of the pilot user friendliness test for four different test modules corresponding to four different sets of operating parameters. The measured quantity is the time for correct entry and verification of the respective parameter set.

measures that are most important for our customers, we interviewed some large industrial customers. According to chemists in analytical labs, three main factors determine their requirements for ease of operation:

- Most instrument operators are nonprofessionals

where is relatively high operator turnover

- The sample mix requires frequent changes of analysis method.

User friendliness is mainly perceived as the effort (that is, time) required by a relatively untrained operator to set

\begin{tabular}{|c|c|}
\hline Task & Measure \\
\hline Installation & $\begin{array}{l}\text { a) Number of Cable } \\
\text { and Capillary } \\
\text { Connections Divided } \\
\text { by Number of } \\
\text { Modules } \\
\text { b) Time for Installation } \\
\text { to First Test Run }\end{array}$ \\
\hline $\begin{array}{l}\text { Verification of } \\
\text { Proper Operation }\end{array}$ & $\begin{array}{l}\text { Time Required } \\
\text { for Checkout }\end{array}$ \\
\hline $\begin{array}{l}\text { Parameter Entry } \\
\text { and Operation }\end{array}$ & $\begin{array}{l}\text { a) Time for Standard } \\
\text { Tasks, Unfamiliar/ } \\
\text { Familiar Operator } \\
\text { b) \% Designations of } \\
\text { Functions Familiar } \\
\text { to Test Persons }\end{array}$ \\
\hline $\begin{array}{l}\text { User Maintenance and Service } \\
\text { (Exchange Cost of Parts) }\end{array}$ & $\begin{array}{l}\text { a) Time per } 1000 \\
\text { Operating Hours } \\
\text { b) Cost per } 1000 \\
\text { Operating hours }\end{array}$ \\
\hline
\end{tabular}

Fig. 7. HP 1050 Series user friendliness measures. 


\section{Design for Manufacturing}

The development of a new product means a challenge for manufacturing, because new processes and manufacturing methods are introduced. The inclusion of three production engineers in the HP 1050 Series development team from the beginning ensured that manufacturing experience was reflected in the design.

JIT (just-in-time) and design-for-manufacturability methods were applied consistently, and had a decisive impact on the structured design of the HP 1050 Series modules. A product-oriented production layout guarantees low production costs, increased productivity, and high flexibility. The number of assembly levels was reduced to three. Thus, assembly, material supply, and order handling could be simplified significantly. Production line personnel were trained to assemble and test the new modules in the prototype phase and were able to provide important suggestions for improvements to the development team. Special emphasis was put on the development of tools and test equipment.

\section{Automated Testing and Networking}

Increased productivity and continuous evaluation of product quality are based on automated tests. HP 9000 Series 300 Computers and HP Vectra Personal Computers are used as controllers to test the HP 1050 Series modules and assemblies during the course of the manufacturing process.

Test data is evaluated both to increase product quality and to supply timely information to control the manufacturing process by statistical methods. A comprehensive network strategy supports the computerized tests in the manufacturing area (see Fig 1).

All LC stations are connected to a central HP-UX station by an HP SRM (Shared Resource Manager) network. At the central station, all test data is collected and stored on a data base. Information is retrieved for monthly quality reports and analyses. Thus, the responsible production engineers can react to problems quickly and properly.

Another advantage of the SRM network is central test program management. This makes sure that all programs are subject to a weekly backup cycle.

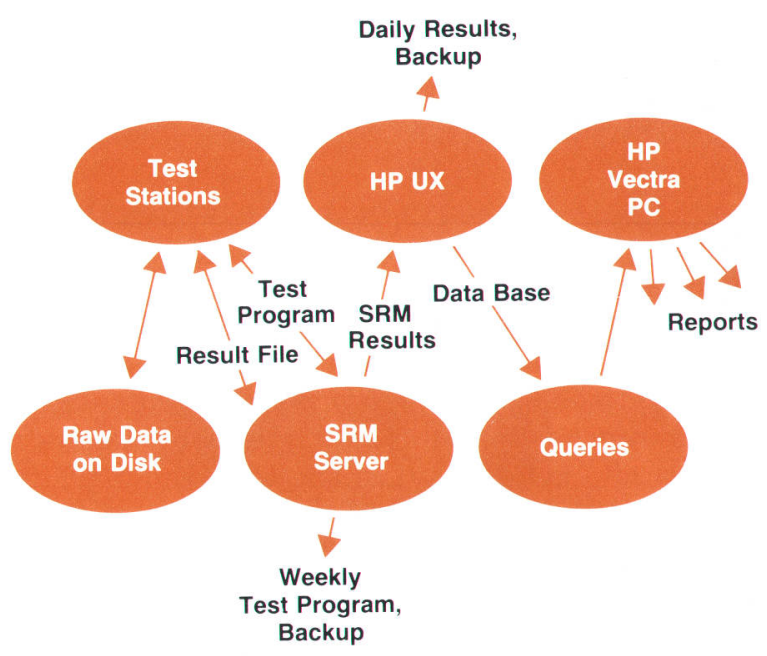

Fig. 1. HP 1050 Series production computer network.

All test stations were already working at the beginning of the production prototype phase. This feedback enabled production engineering and R\&D to receive and evaluate important information on manufacturability and testability at an early stage. This made it possible to improve the tests and increase their efficiency by continuous development.

\section{Continuous Flow Manufacturing (CFM)}

A prerequisite for continuous just-in-time (JIT) production is a material-flow-oriented line layout (Fig. 2). The material for HP 1050 production is conveyed directly from the stockroom to the line by a material elevator. Thus, long and awkward material flows are avoided and the location of the material is known at all times.

The straightforward material flow is also supported by the material list structure for the HP 1050 modules, which has only three levels at most.

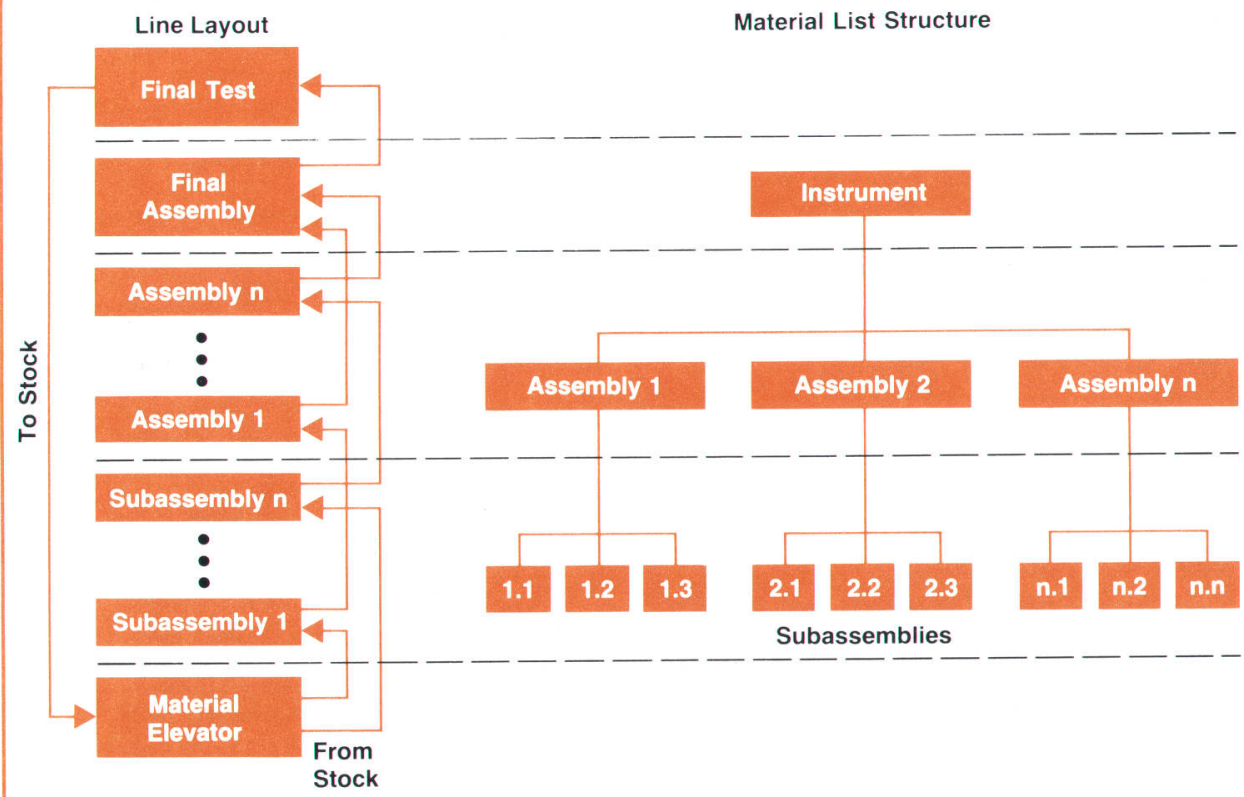

Fig. 2. The line layout corresponds to the material list structure. 


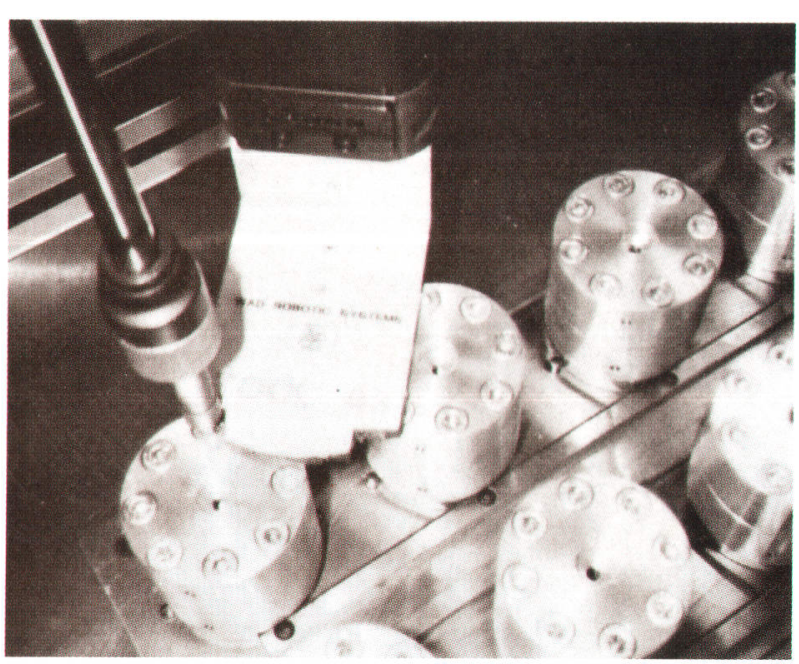

Fig. 3. Installing a damper screw.

\section{Robotics}

In the HP 1050 pumping system, the state of the membrane between the damper head and the damper body determines the performance of the high-pressure damper. To avoid placing this membrane under incorrect tension, it is necessary to tighten the eight screws of the damper in different sequences with increasing torques. The use of the high-pressure damper in both the HP 1090 and the HP 1050 led to a drastic increase in the number

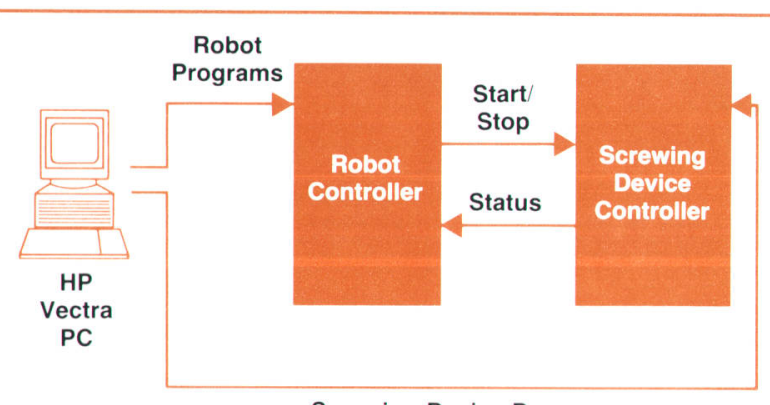

Screwing Device Programs

Fig. 4. Robot station data flow.

of units. To maintain quality, the manual screwing procedure formerly used had to be abandoned.

To ensure a stable level of quality, the tightening is now done by an electronically controlled screwing device connected to a robot (see Figs. 3 and 4). This robot is also used for preassembly of the high-pressure damper. The programs for the screwing device and the robot were written on an HP Vectra personal computer and are retrieved from the hard disk as needed. By monitoring the screwing procedure -in particular the torque, the angle of rotation, and the screw-in depth-each irregularity of the damper and screw threads can be detected.

Heiko Breckwoldt Manfred Seitz

Production Engineers Waldbronn Analytical Division up and run an analysis.

Based on this information, we developed a test method. It consists of defining standard tasks for the unit under test and measuring the time required to perform these tasks by test persons without any operating experience on the unit under test, relative to very experienced operators. A pilot test with 10 test persons was then performed on an LC workstation, to find out whether such a test is feasible and gives meaningful results, and what test procedure would work best, including:

- How much familiarization for test persons is optimal (15 minutes)

- Whether to do video monitoring or not (no)

- How to measure time (monitoring by an observer)

How to record specific operating problems.

Fig. 6 shows some results of this pilot test. Since we observed quite a wide range between the quickest and the slowest first-time users, we decided to use both the average ratio and its range as our measures. Later tests showed that the range (slowest minus quickest first-time user, relative to experienced user) may be an indicator of the product's learnability. ${ }^{*}$ In addition to these measures, the most frequent operating problems experienced by the test persons were recorded as a basis for improvements of the user interface.

The next step was to calibrate our test results against user perceptions of how user friendly the LC workstation was. Several hundred users were surveyed, with regard to the parameters tested, on how easy to use they perceived the workstation to be. The survey also contained questions that helped us define an average operator profile. This was important in selecting the right test persons. The survey results and the test results together gave us the basis for setting design goals for user friendliness of the HP 1050 Series. In addition, several other factors from our brainstorming list were included in the HP 1050 Series user friendliness measures (Fig. 7).

The test procedure was successfully used by the HP 1050 detector design team during user interface prototyping to improve the user interface. Fig. 8 indicates the significant progress in user perception that was achieved by this process.

Finally, a test of the complete system was also done with 13 test persons. Figs. 9 and 10 show some results of these tests. In most test categories, the HP 1050 system meets its design goals. We will complete the test with a user survey identical to the one answered by the test persons after finishing the test. This calibration step will provide further data to compare test results with user perceptions, and will allow us to make further refinements in test methods and goal setting.

\section{Acknowledgments}

The authors thank Helmut Roessler for doing most of the strife testing and for monitoring most of the user friendliness testing. We also thank our product marketing people for their help with selecting the customer sample for the customer survey. The data in Fig. 8 was contributed by the detector design team. 

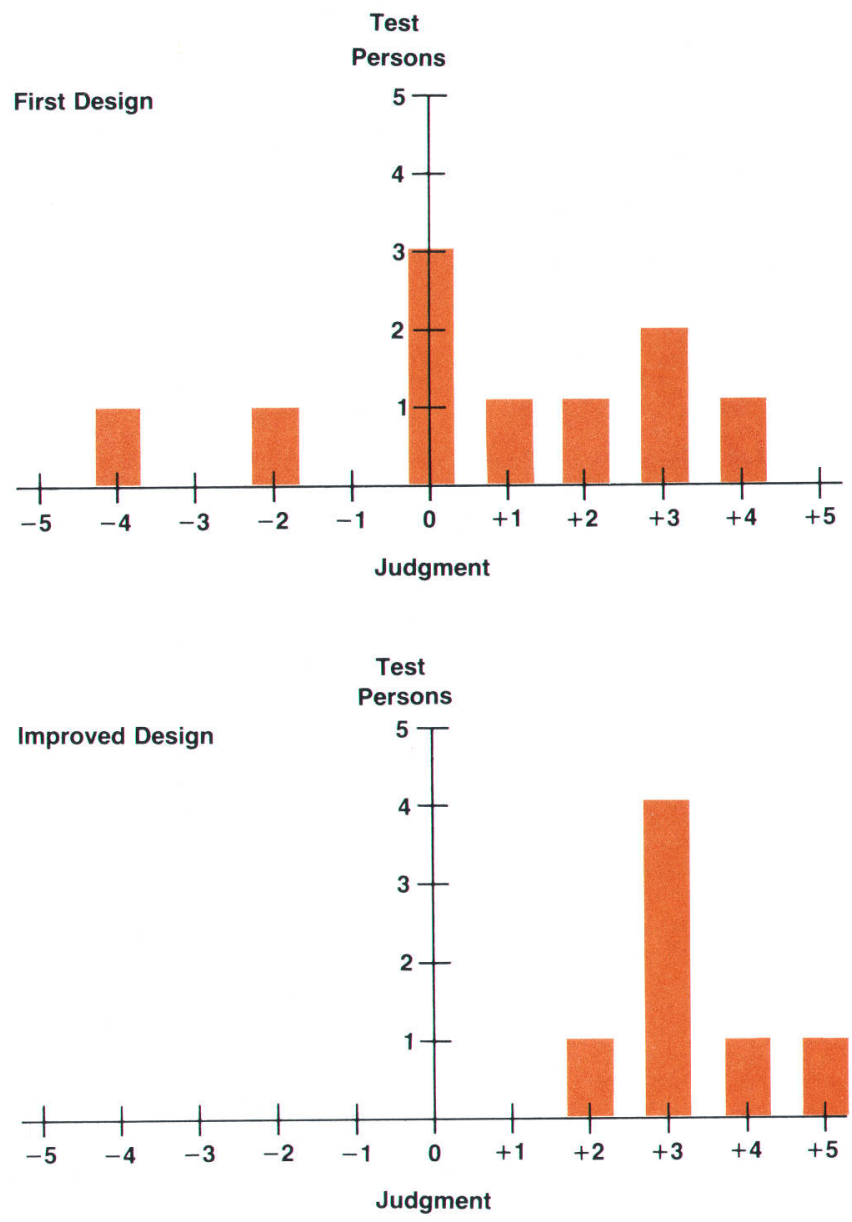

Fig. 8. Judgment of test persons on the friendliness of the dialog logic of the HP 1050 Series diode array detector before and after improvement of the user interface by prototyping.

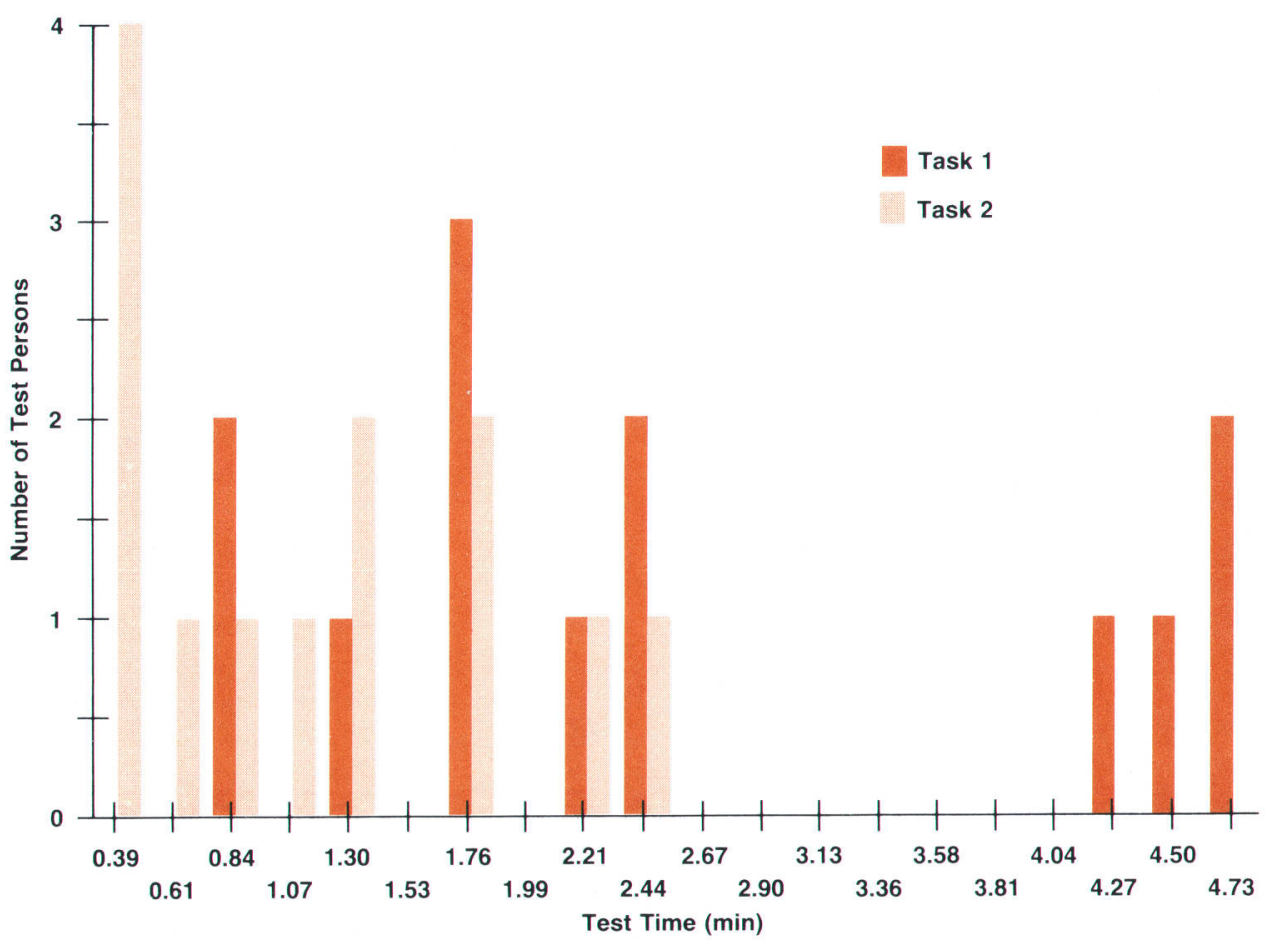

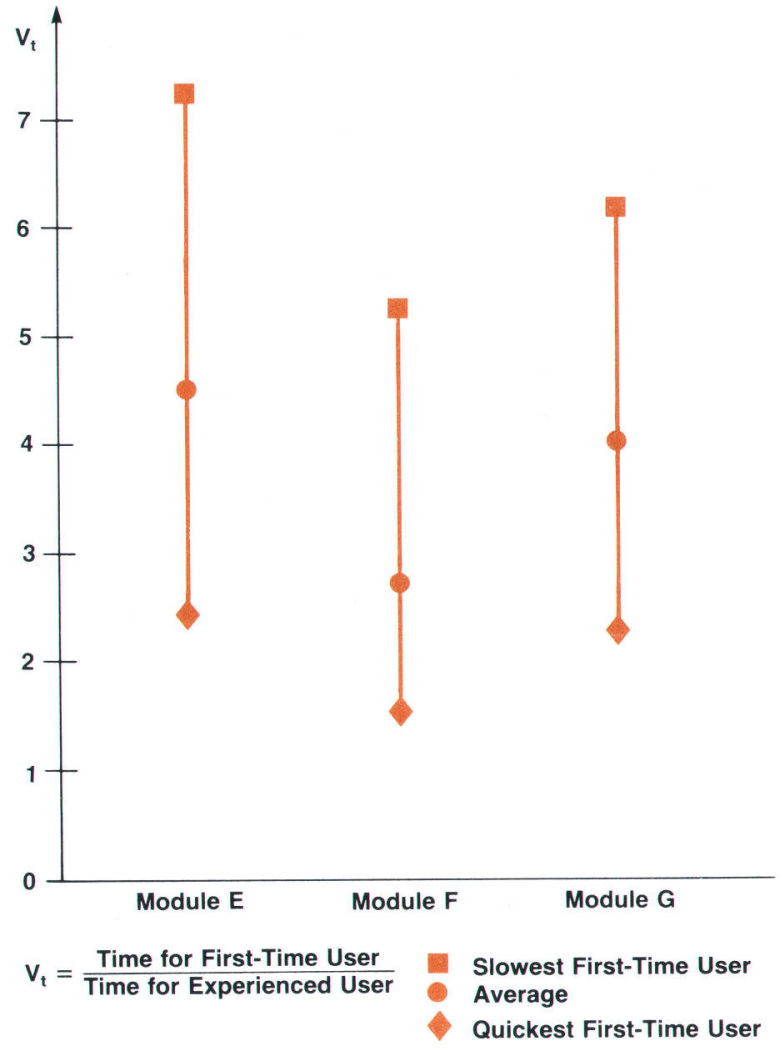

Fig. 9. Results of part two of the HP 1050 Series user friendliness test.

\section{References}

1. E.O. Codier, "Reliability Growth in Real Life," IEEE Transactions on Reliability, Vol. R-36, no. 1, April 1987.

2. P.D.T. O'Connor, Practical Reliability Engineering, Second Edition, John Wiley \& Sons, 1985.
Fig. 10. Results of part three of the HP 1050 Series user friendliness test for the pump module. The large span of times required by the test persons for task 1 is significantly reduced for task 2 , which was similar to task 1 . This is an indication of the learnability of the product. 


\title{
A Compact, Programmable Sample Injector and Autosampler for Liquid Chromatography
}

\author{
The HP 1050 Series autosampler is capable of manual or \\ automatic injection from up to 119 sample vials at injection \\ volumes up to 2000 microliters.
}

by Wolfgang Kretz and Gerhard Ple

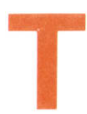

HE HP 1050 SERIES AUTOSAMPLER MODULE (Fig. 1) is a compact, stackable, fully programmable, microprocessor-controlled stand-alone unit designed to inject liquid samples automatically onto the column in a liquid chromatographic system. It performs routine analysis for quality control as well as analysis method development in research laboratories. It can either work in an HP 1050 Series LC system environment or together with HPLC modules from other instrument suppliers.

The autosampler is programmed via its front-panel keypad. A two-line display prompts the user in setting parameters and provides the required status information. The standard sample capacity is 21 sample vials, which can be randomly accessed for analysis. If required, the number of available sample locations can be expanded to 119 by adding a dedicated sample tray with a robot-like manipulator. This tray also allows temperature control for its vial positions.

The standard autosampler allows injection volume settings from $0.1 \mu \mathrm{l}$ through $100 \mu \mathrm{l}$ without the need to change any hardware. Optionally, the injection volume can be extended to $2000 \mu \mathrm{l}$ with a hardware modification.

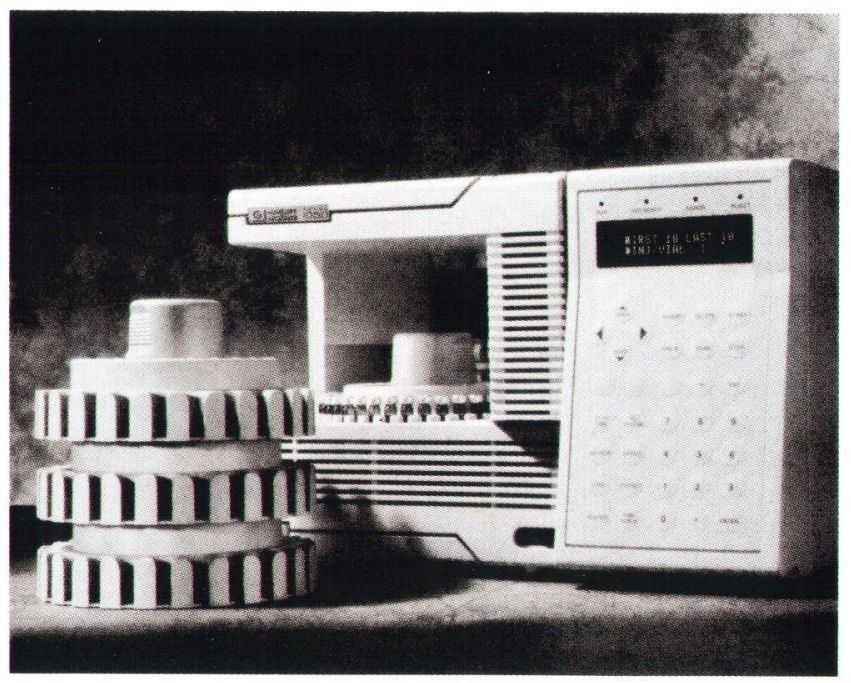

Fig. 1. HP 1050 Series injector and autosampler module. The standard tray holds 21 sample vials.

\section{Design Goals}

From the beginning the main design philosophy was to offer our customers an instrument with all major functions at a moderate price. When sample throughput or other requirements grow, the system can also grow, up to the highest degree of automation with a robotic system serving the autosampler. The main design goals were to provide:

- A stand-alone module capable of working in any HPLC system

- A simplified hydraulic path to reduce maintainance and increase reliability

- A design that conforms to HP 1050 Series common design guidelines for minimum footprint

- Upgradability to adapt to changes in customer demands

- A wide injection volume range to minimize the need to modify the instrument

Maximum use of standard components to reduce cost of ownership

- Access for a robotic vial handler

a Fast random access to minimize the time required to carry out an injection

No extra wash mode, thereby simplifying operation

- A maximally safe design with built-in leak drainage and leak detector.

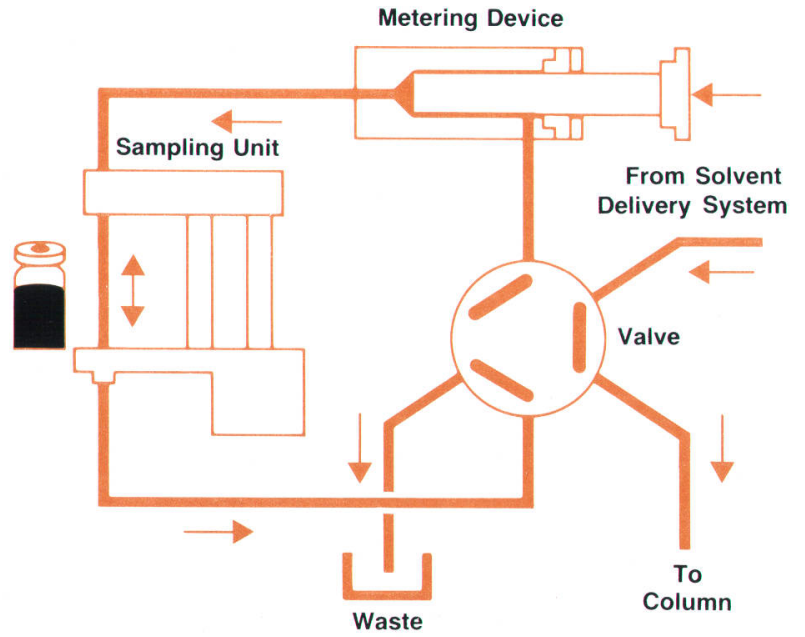

Fig. 2. Prepare-to-inject condition. 


\section{Mainframe}

The autosampler follows the general design philosophy established for the HP 1050 Series. However, unlike the other modules, the autosampler has a large opening at the left front corner. This is to make the operating area easily accessible to the user, so that vials and trays can be inserted and removed very conveniently and simply. There is also access for the optional 100-vial tray and for a robotic system to manipulate the vials and trays.

All HP 1050 modules are required to have random stackability, so special attention was paid to the mechanical stability of the autosampler module. As in the other modules, there is strict separation of the chromatographic area and the electronic area. Manufacturability, electromagnetic compatibility, leak handling, and accessibility for repair were also important considerations throughout the entire development cycle.

\section{Theory of Operation}

The hydraulic path of the standard autosampler consists of three main assemblies: the six-port valve, the metering device, and the sampling unit. The six-port valve switches the solvent path to go through or bypass the other elements. The sampling unit acts as the interface to the vial. It transports the selected vial to the needle position, where the needle can move into the vial. The metering device then draws the programmed amount of sample into the system.

A complete injection cycle starting with an initialized system works as follows. The first step is the prepare-to-inject step (see Fig. 2). The six-port valve is switched to the bypass position so that the pump delivers flow directly onto the column and the remaining injector elements-metering device, sample loop, needle, and seat capillary-are bypassed. The liquid volume in this area, which is under a high system pressure of up to 400 bar before switching, can expand through the waste outlet of the valve. The metering device plunger is moved to its front (home) position, displacing a small portion of solvent to waste.

The next step is the load-sample step (see Fig. 3). In the sampling unit the needle is lifted and the sample tray is rotated until the selected vial is directly under the needle. The needle is lowered into the sample liquid in the vial to allow the metering device to draw up the desired volume by moving its plunger back a certain distance. The needle

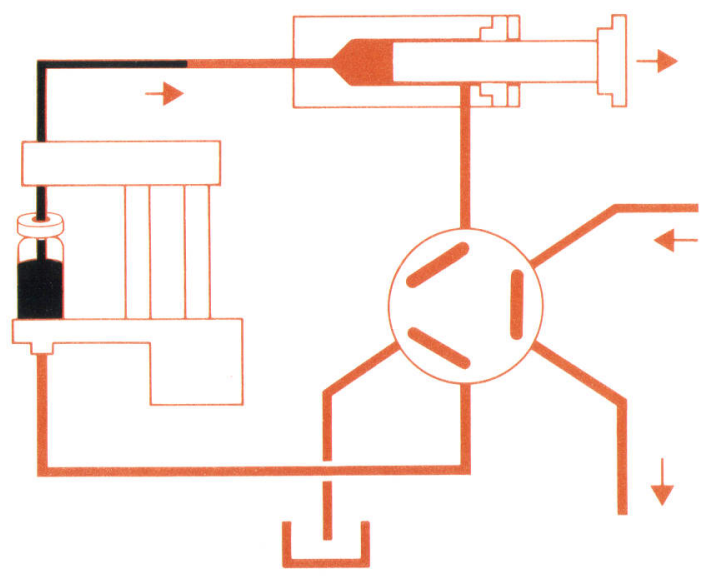

Fig. 3. Load-sample condition.

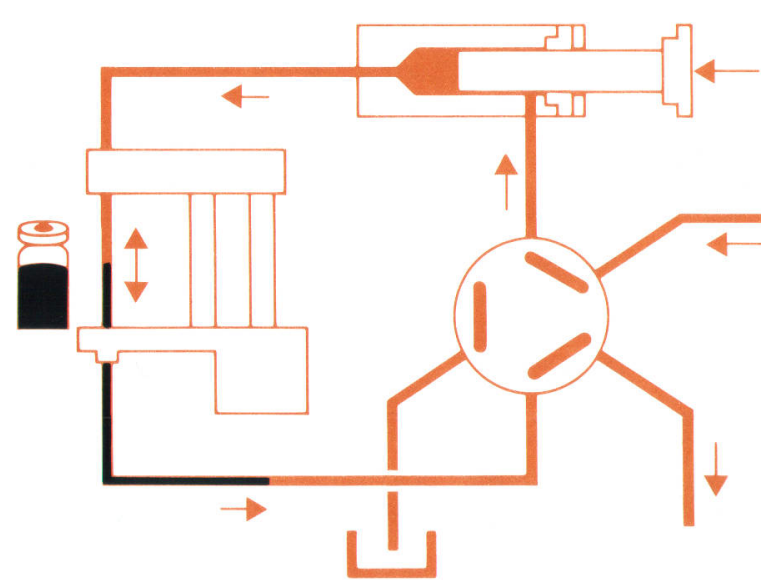

Fig. 4. Inject-and-run condition.

is then raised again, the tray is moved to its home position, and the needle is moved onto the seat to close the sample loop.

The final step is the inject-and-run step (see Fig. 4). The six-port valve is switched to its mainpass position, which directs the flow back through the loop, which now contains the sample volume. The solvent stream transports the sample onto the column, and separation begins. This is the beginning of the analysis. In this hardware condition, which is also the starting condition for the next injection, all major performance-influencing hardware is flushed by the solvent flow. Therefore, no additional flushing hardware and procedures are required.

\section{Extended Injection Volume}

The standard instrument allows injection volumes up to $100 \mu \mathrm{l}$, which is the metering device volume. In some cases, larger volumes are required. This can be achieved by repeatedly drawing and storing partial volumes in the capillary connecting the seat to the valve. This mode of operation is called the multiple-draw mode. Before this can be done, the capillary must be extended with a larger one that can hold the largest volume to be injected (see Fig. 5).

In the multiple-draw mode the load-sample step is mod-

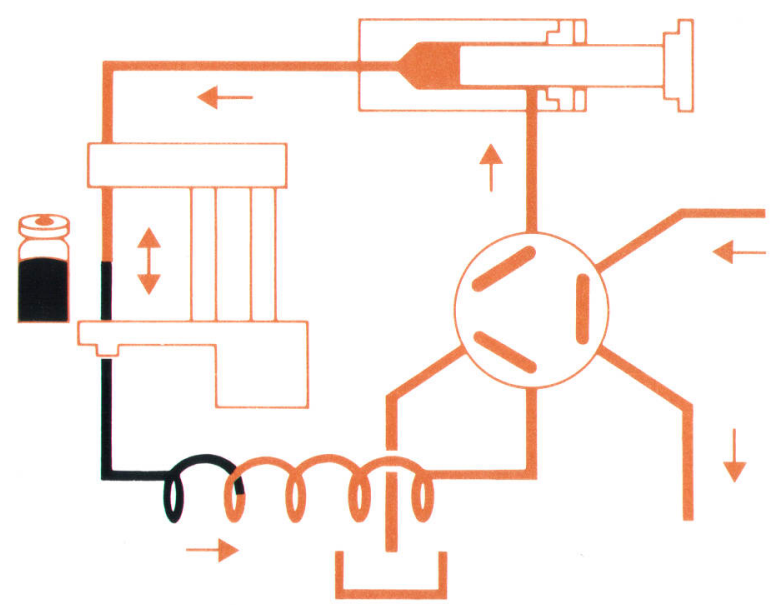

Fig. 5. Loading a partial sample into an extended seat capillary. 
ified. After drawing up and reseating the needle, the plunger is moved to the front position to place the $100-\mu \mathrm{l}$ sample volume, or "plug," in the seat capillary. Then more sample is drawn from the vial and another 100- $\mu$ l sample plug is placed in the capillary. This is repeated for the required number of $100-\mu \mathrm{l}$ partial volumes. The last partial volume remains in the standard loop and the conventional inject-and-run step follows.

\section{Sampling Unit}

The sampling unit (Fig. 6) has two major functions. First, it opens and closes the sample loop by means of a movable needle and a fixed seat, which form a seal that can withstand high pressure (up to 400 bar or 5800 psi). Second, it stores sample vials and moves the desired vial into the inject position.

The stainless-steel needle, fixed in an arm, is moved up and down by a stepper-motor-driven leadscrew and guided by bushings on a shaft. The lower needle position is determined by a multifunctional optical sensor and a spring which is loaded when the needle moves onto the seat. This spring applies the required force to ensure a leakproof seal of the needle on its seat with zero motor current. The same optical sensor is also used to determine the upper needle position to ensure enough clearance for vials to be moved underneath. In addition, there is a missing-vial sensor, which is actuated only if a vial is present when the needle moves down to the draw position.

The sample vials are held in an internal tray with 21 positions. The tray is manufactured as a precision plastic molded part. It can be removed very easily from the instrument to be loaded with sample vials. It can also be used as a sample container to be stored in a refrigerator. Several trays can be stacked easily and used to store vials on the lab bench. The driving mechanism for the sample tray is a stepper motor with a quadrature encoder for a position feedback sensor. The encoder resolution is 2000 counts per revolution, which corresponds to an angle of 0.18 degree. This allows very precise and reproducible rotation of the tray to the programmed vial location starting from the home position. Correct vial positioning eliminates the possibility of needle damage resulting from mispositioning caused by accident or user interaction during rotation. In addition, the absolute positioning ensures that the correct vial is selected. The home position and presence of the tray are checked by a Hall-effect sensor which is actuated by a small

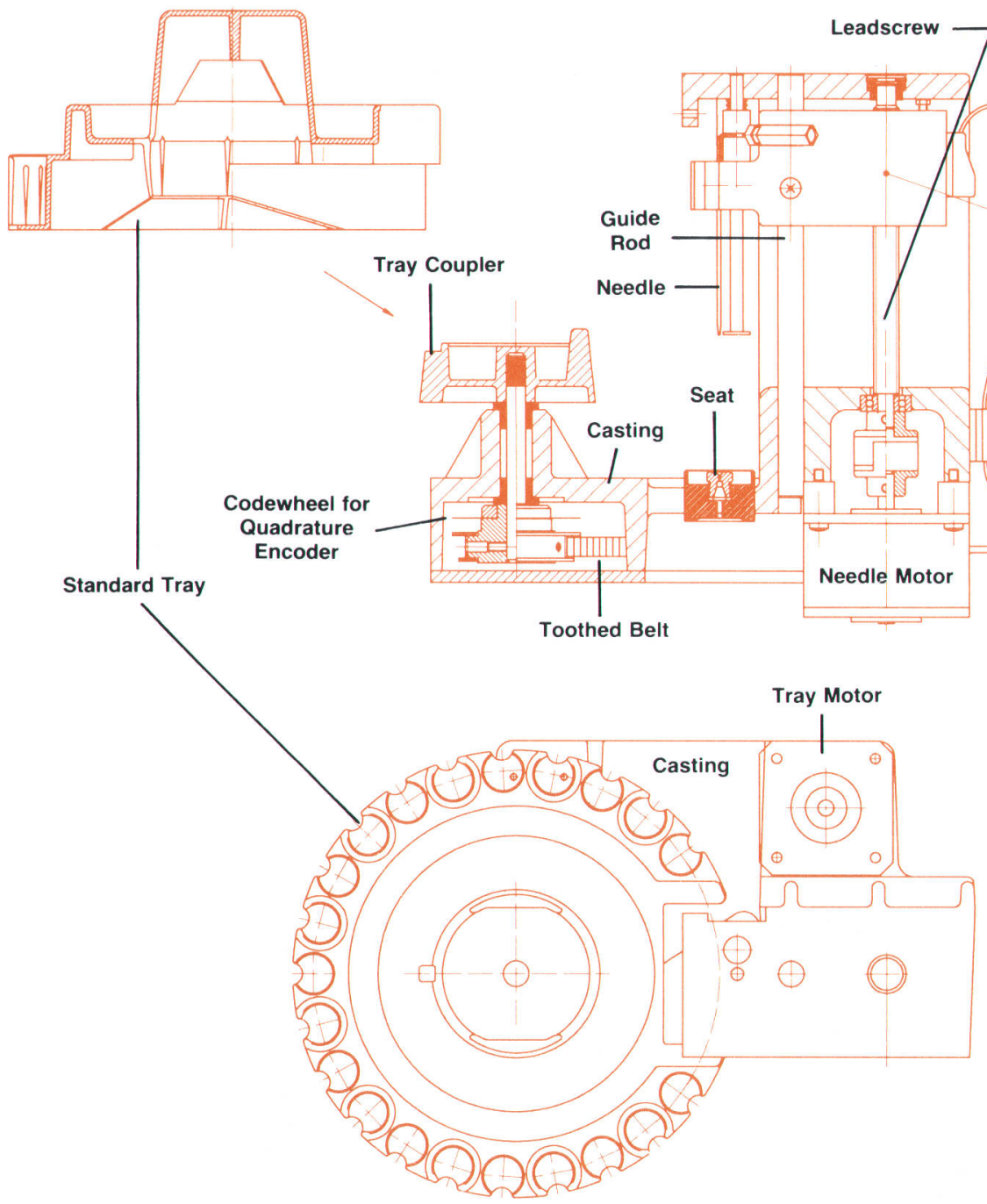

Fig. 6. Cutaway view of the sampling unit. 
magnet fixed in the tray. By evaluating the polarity of the sensor signal, two types of trays can be distinguished. All functional parts are integrated onto a machined aluminum permanent-mold casting.

\section{Metering Device}

The metering device shown in Fig. 2 is a key element that strongly contributes to the precision of an autoinjector system. It must meter exactly the desired volume to be injected. The metering device consists of the analytical head assembly and the metering drive (Fig. 7). The analytical head assembly connects with the sampling needle and the solvent delivery system. It contains a plunger to draw up the required amount of sample and subsequently inject it onto the LC column. The metering drive drives the plunger.

To draw up a sample, the metering drive displaces the plunger in the head a certain distance. The metering drive is a high-precision linear actuator with a stepper motor driving a leadscrew via pulleys and a toothed belt. It is integrated in a carrier made of sheet-metal parts and aluminum permanent-mold castings. A vane mounted on the guiding nut of the leadscrew interrupts an optical sensor in the farthest stroke position and defines the home position.

Since the analytical head assembly has to withstand the high system pressure of up to 400 bar, its design is very similar to the solvent pump head. The sapphire plunger and its seal are the same parts used in the HP 1050 solvent pumps. When drawing up sample, the plunger is forced by a spring to follow the leadscrew exactly without backlash. The leadscrew and the plunger are decoupled by a ball, which transfers force. This design compensates for mechanical tolerances and increases the lifetime of the plunger seal. The overall resolution is $7 \mathrm{nl}$ displacement of the plunger with one step of the motor.

\section{Electronic Design}

In addition to the electronics found in all HP 1050 Series modules-power supply, microprocessor board, keyboard, and display-the autosampler contains module-specific electronics to support the injector hardware. Fig. 8 shows the autosampler electronic block diagram. The central motherboard, which interconnects all of the boards in the card cage, also has connectors for the electronic components located in the hardware area.

The standard configuration has two additional boards. The first board controls the metering drive and the six-port switching valve, while the second board interfaces to the sampling unit. The first board also carries the injector-specific firmware on a small piggyback board. When the optional 100-vial tray is installed, a third board is added. The module-specific boards contain the stepper motor drivers and control logic to run the motors at speeds in the 1000steps/second range while also monitoring and responding to the limit sensors. This design reduces the real-time hardware-servicing requirements on the microprocessor and frees it from a lot of simple, periodic work.

\section{0-Vial Tray Integration}

The 100-vial sample tray (Fig. 9) was first introduced to increase the sample throughput of the HP 7673A Gas Chromatograph injector. This tray was developed by HP's Avondale Division. Because of its modularity, we found the tray

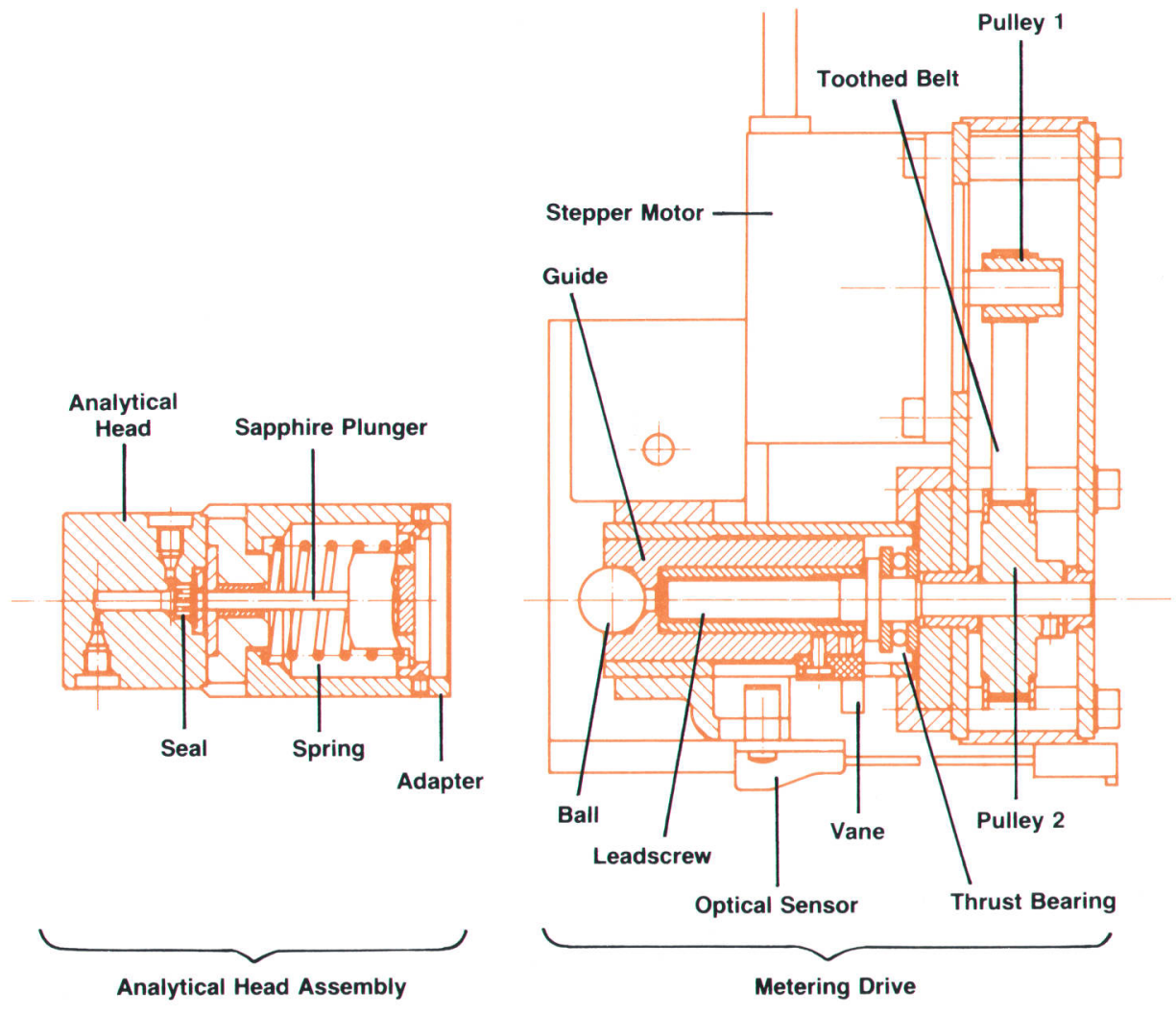

Fig. 7. Cutaway view of the metering device. 


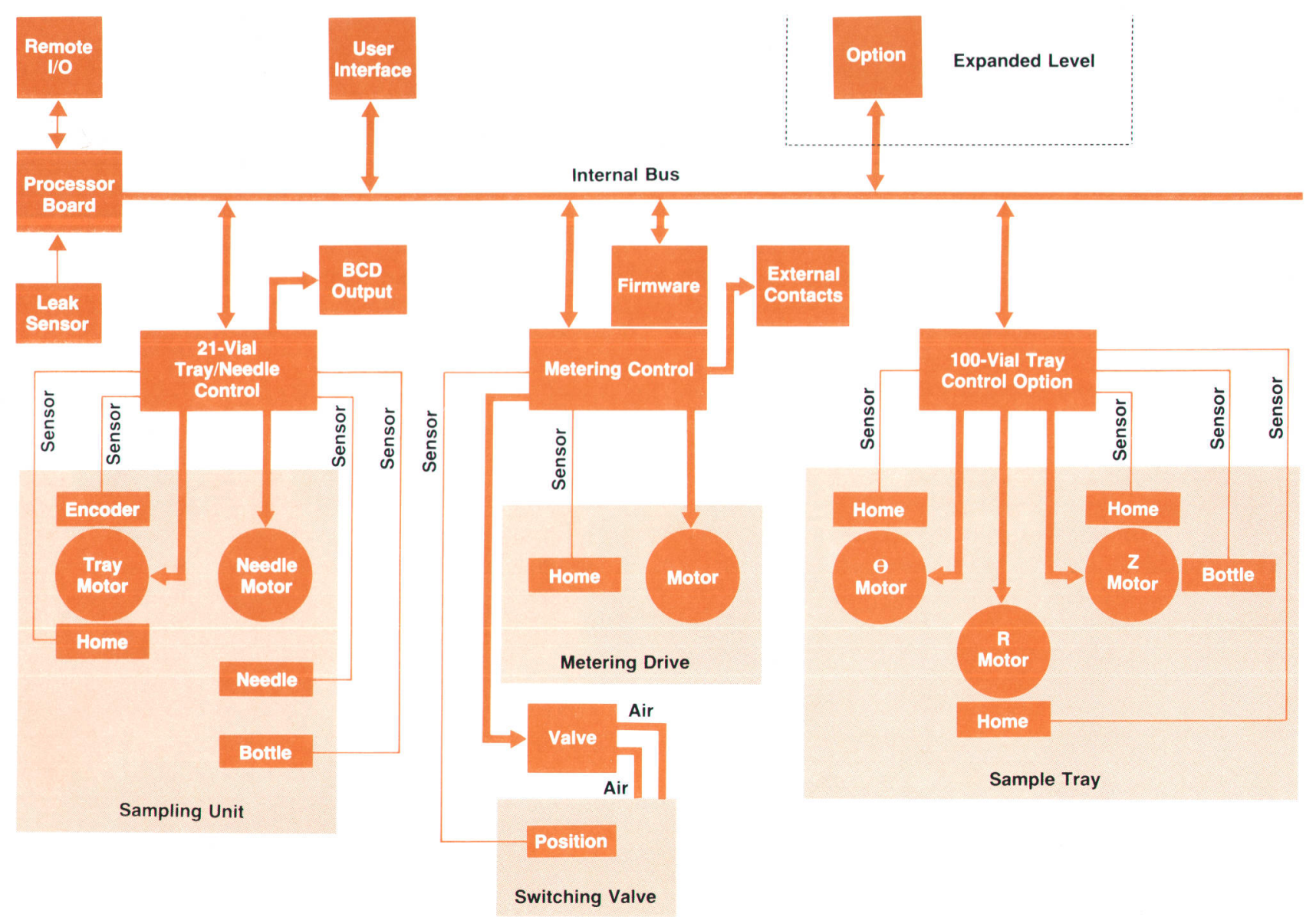

Fig. 8. Autosampler electronic block diagram.

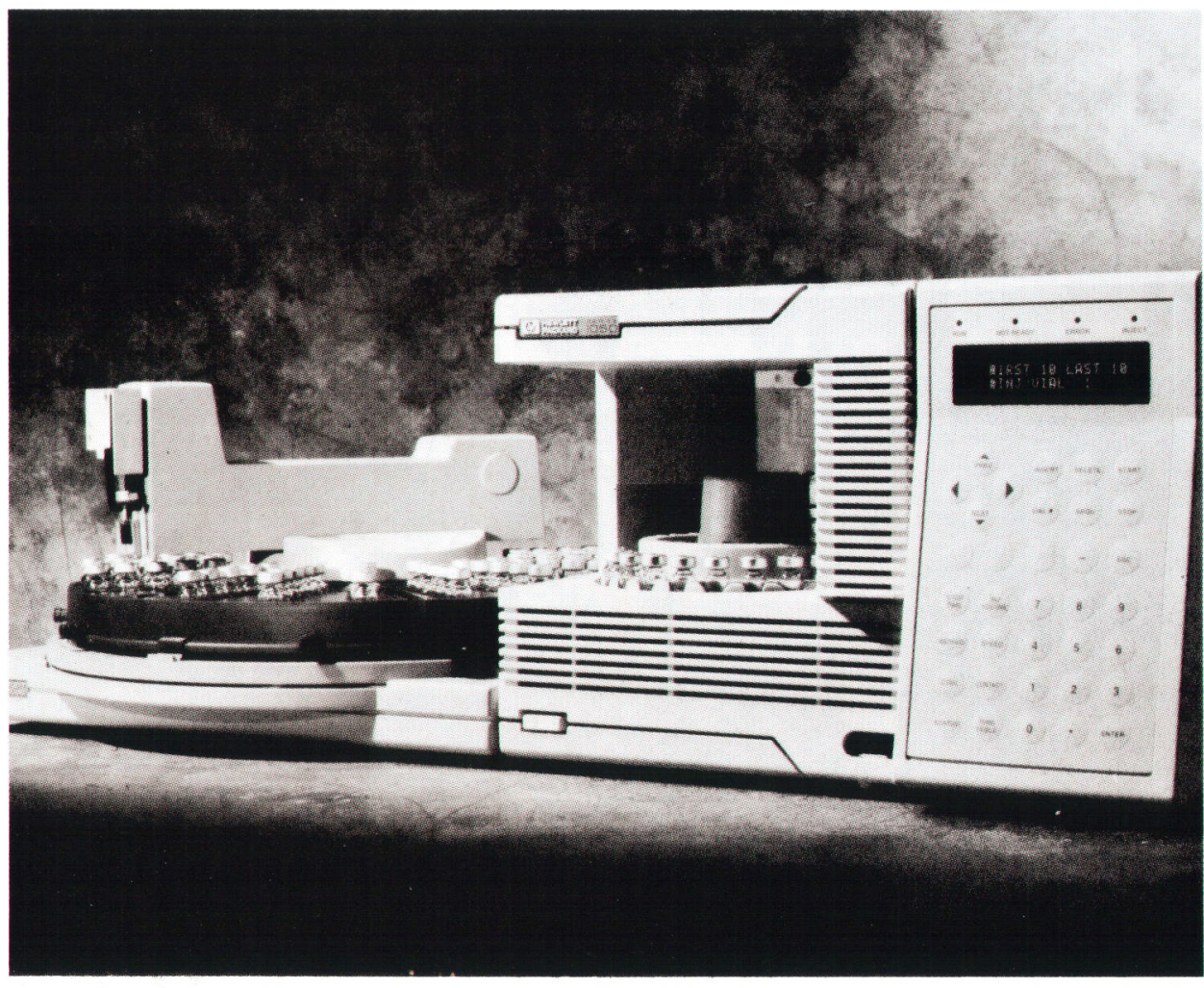

Fig. 9. Injector with 100-vial tray. 
to be an ideal way to increase the sample capacity of the HP 1050 injector and provide temperature control of the vials. The opportunity to leverage an existing, available product instead of duplicating the design effort was an additional benefit.

The tray offers storage, transportation, and temperature control of 100 sample vials. The vials are placed in four removable quadrants. A circulating bath can be connected to fill and drain adapters at each quadrant. The transport mechanism is a small manipulator consisting of an arm and a gripper operating in cylindrical coordinates. The manipulator is mounted on top of the drive mechanism located in the center of the tray base. To transport a vial, the arm moves radially and turns around the tray axis to place the gripper close to the selected vial position. The gripper moves vertically down and the vial is engaged at its neck. After it is raised, the vial can be placed anywhere within the arm's accessible area.

The 100-vial tray is adapted to the HP 1050 autosampler by a compound design (Fig. 10). Sheet-metal parts used for the support ensure proper positioning and the required stability. A polyurethane foam part between the two sheetmetal parts spans the distance and adapts the tray's appearance to the HP 1050 industrial design. This support assembly is attached to the autosampler base with only four

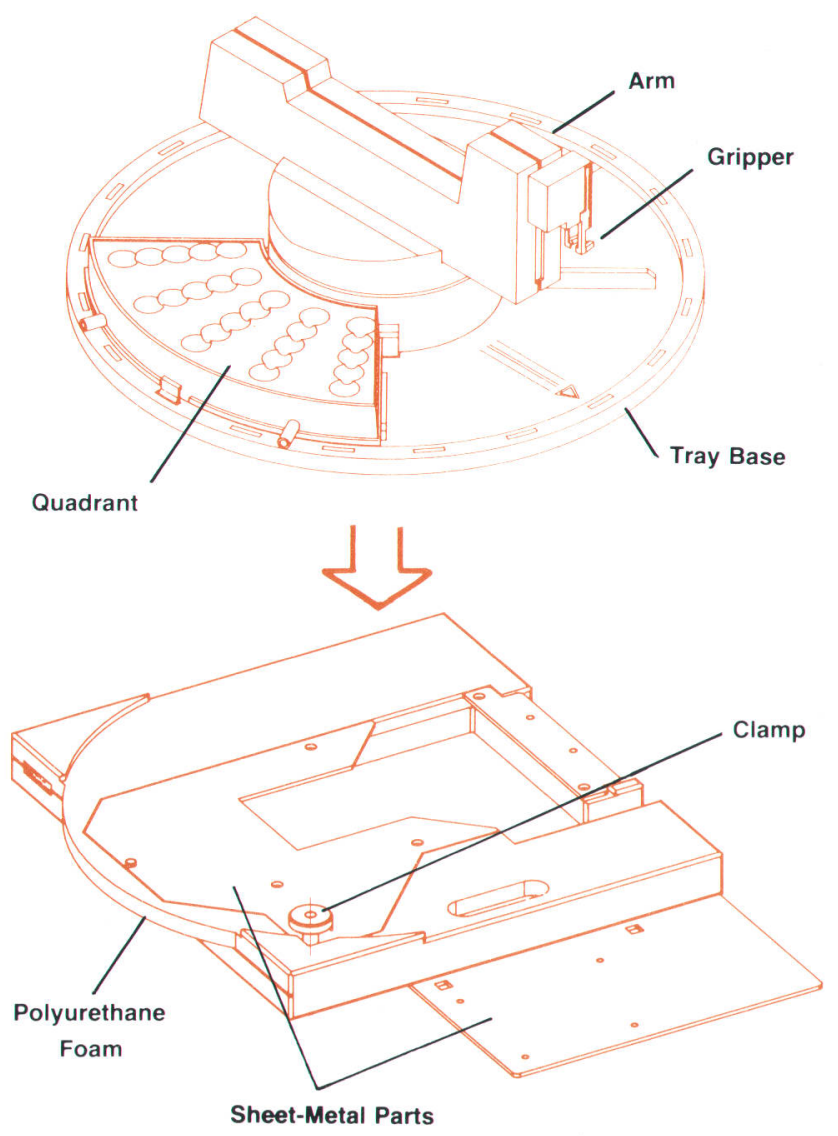

screws. The tray slides into the bracket to its defined position and is fixed with a spring-loaded clamp. The tray is controlled by an additonal printed circuit board in the cardcage.

If the 100-vial tray is installed, the user can specify not only internal vials but also vials within the external tray area. In the internal tray, a transfer position is defined for vial exchange. For a sample injection from an external vial the arm picks up that vial and places it into the transfer position of the internal tray. Then a standard injection is done using the vial in the transfer position. After injection, the vial can be returned to the external tray or kept for additional injections.

\section{Automation Capabilites}

The major use of an automatic injector with autosampler is to permit an LC system to run unattended, doing a series of analyses in a predefined way, say overnight or during the weekend. The HP 1050 autosampler offers two modes of automation. One mode, which is very easy to set up, can be used to analyze a set of samples under preset conditions. The user only has to specify the first vial, the last vial, and the number of injections per vial. Once started, the system analyzes the specified range automatically. There is no chance of changing analysis parameters, but this mode is very useful for doing a few injections rapidly.

A more sophisticated way of automating an LC system is to use the full sequence capability of the autosampler. This mode allows programming of parameter changes and periodic insertion of calibration injections. There are more parameters to be set up, but the versatility is high. The overall analysis sequence is built up by chaining parameter sets. A set always works with the same analytical conditions. Chaining of up to nine sets is possible, and sets can be disabled to prevent their execution. A set contains the following parameters:

$\begin{array}{ll}\text { seti } & \text { on/off } \\ \text { waittime } & \mathrm{xmin} \\ \text { first vial } & \mathrm{xxx} \\ \text { last vial } & \mathrm{yyy} \\ \text { \#of inj } & \mathrm{zz} \\ \text { inj meth } & \mathrm{m} \\ \text { overlap } & \text { on/time } \\ \text { calib } & \text { off/multi/alter } \\ \text { first calv } & \mathrm{ccc} \\ \text { last calv } & \text { ddd } \\ \text { \# of inj } & \text { ee } \\ \text { after } & \text { ff vials/runs }\end{array}$

Fig. 11 shows an example of a set that includes periodic recalibrations out of calibration vials (calv) and results in the following series of injections (ci stands for calibration vial $i$ and si stands for sample vial $i$. The calibration mode is set to multi.):

start-c1-c1-c2-c2-c3-c3-s4-s5-s6-s7-s8-s9-c1-c1-c2-c2-c3-c3-s10-s11s12-s13-s14-s15-c1-c1-c2-c2-c3-c3-s16-s17-s18-s19-s20-s21-c1-c1c2-c2-c3-c3-finished

Fig. 10. HP 1050 Series support for the 100-vial tray. 
FIRST CALV 1

LAST CALV 3

INJ/CALVIAL 2

AFTER 6 VIALS

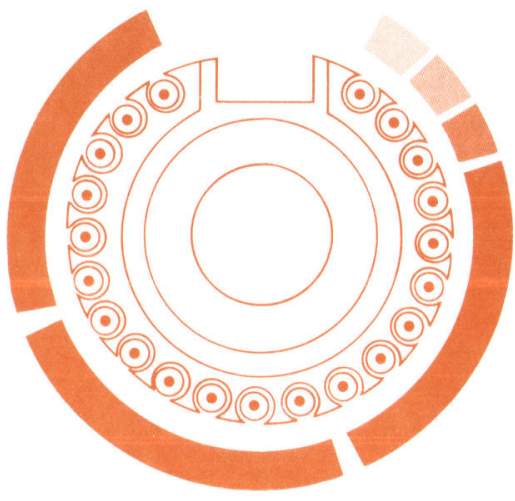

Fig. 11. Typical setup of sequence and vials for unattended analysis.

\section{Acknowledgments}

The HP 1050 Series autosampler is a very versatile and powerful LC module that contributes much to free the chemist in the lab from boring standard work. The completion of the project was only possible with the enthusiasm and the steady work of many people, in particular the R\&D team. Many thanks to Hans-Peter Zimmermann who worked on the sampling unit, plastic parts, and integration of the external tray, to Karl-Heinz Kraemer who worked on the entire electronic hardware, to Herbert Anderer who programmed the automation features, to Fritz Bek who worked on plastic and sheet-metal parts and programmed the user dialog tables, and to Michael Leiber who programmed the 100-vial tray control. Very helpful in getting the 100-vial tray integrated was the support of the people at the HP Avondale Division. We would especially like to thank John Poole, Ernie Zerenner, and Paul Welsh for their time spent in discussions and their inputs. 


\title{
Flexible, Precise Solvent Delivery for Liquid Chromatography
}

\author{
The HP 1050 Series LC pump merges reliable, known \\ technology with powerful control capabilities that \\ compensate for solvent properties and physical side \\ effects. A custom IC implements the motor and pump \\ control functions.
}

\section{by Fred Strohmeier and Klaus Witt}

IKE ALL HP 1050 SERIES MODULES, the pump module (Fig. 1) is a microprocessor-controlled standalone unit with full programming capabilities. It is compact in size and stackable with other HP 1050 Series modules to form a chromatographic system.

The module can be used for analysis method development in research laboratories and for routine analysis in quality control. It can work either in HP's system environment or together with instruments from other manufacturers.

Parameter setting and programming are done using the functional keyboard on the front panel. A two-line display prompts the setting of parameters and provides feedback by displaying parameter values.

The instrument is available with two different levels of complexity to meet the needs of customers' applications. At the entry level is the isocratic version, which can only pump one liquid from a bottle. The more flexible quaternary version is able to blend liquid from up to four different bottles in a selectable and time-programmable mixture.

The main design goals for the pump were moderate cost and flow performance that does not limit the chromatographic applications. Because the range of chromatographic

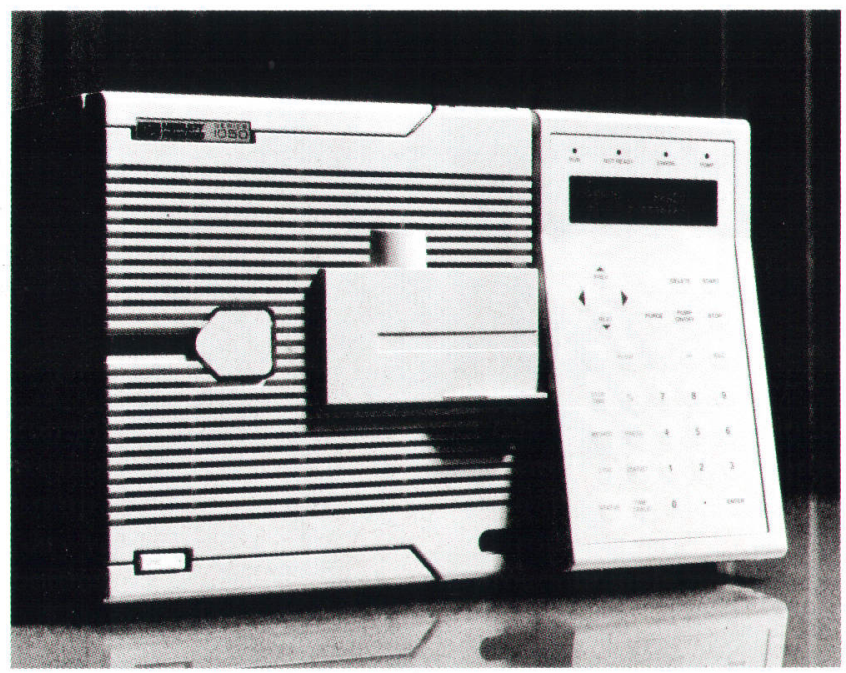

Fig. 1. Isocratic HP 1050 Series LC pump module. applications is wide, the flow rate of the liquid delivered to the column is adjustable over a wide dynamic range. The HP 1050 pump is designed for a factor of 10,000 between the lowest and the highest flow rates. It delivers up to $10 \mathrm{ml} / \mathrm{min}$ in $1-\mu \mathrm{l} / \mathrm{min}$ increments.

\section{Pump Design Options}

Although the flow rate of an LC solvent delivery pump should be adjustable, it is very important that once selected, the flow rate be kept constant. If the flow rate through the separation column fluctuates, variations in the retention times and the areas of the chromatographic peaks will occur. Since the peak areas are representative of the concentrations of the separated sample components, fluctuations in the flow rate would impair the accuracy and the reproducibility of quantitative measurements. The various detector types show different sensitivities to flow rate variation. Commonly used detectors in liquid chromatography are absorption detectors, fluorescence detectors, electrochemical detectors, and refractive index detectors.

Some pumping systems, such as reciprocating pumps with single pistons, have inherent flow rate variations because the piston delivers only during a portion of the pump

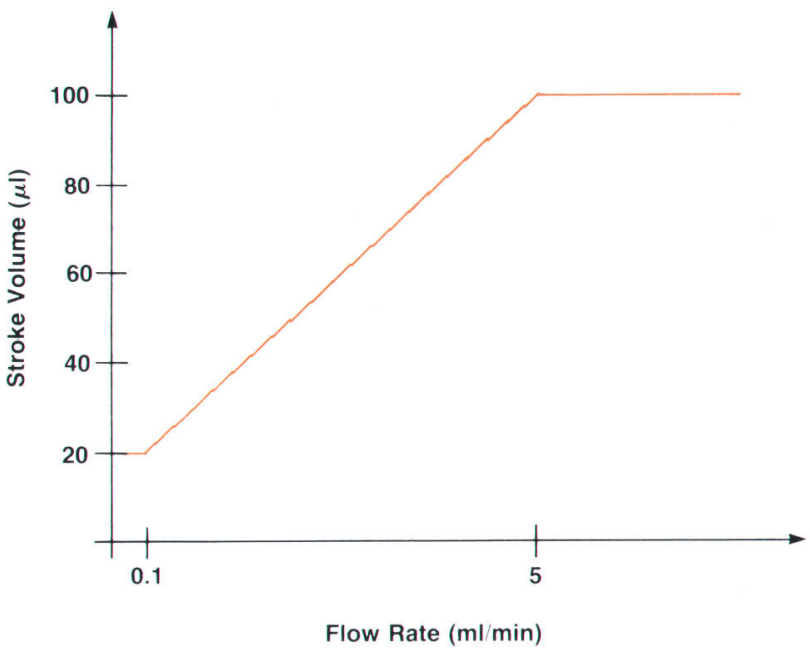

Fig. 2. Stroke volume as a function of flow rate, showing the variable stroke capability of the HP 1050 pump. 
cycle. Hydraulic capacitance is used downstream from the pump to filter out most of the $100 \%$ pump ripple. To reduce the pump pulsation, it is possible to use a dual-piston pump having two interconnected pump heads, each with a reciprocating piston. The pistons are driven by a cam and a camshaft with a predetermined phase difference so that one piston is delivering while the other is drawing, and the resulting flow rate is comparatively smooth. This type of pump is used in the HP 1050 pump module.

Pumps using techniques other than reciprocating pistons (e.g., peristaltic, centrifugal, or gear pumps) are seldom used in high-performance liquid chromatography because the flow rate is also expected to be constant despite pressure variations of up to 400 bar, which is about 5800 psi.

At the high pressures encountered in high-performance liquid chromatography, compressibility of the solvents becomes noticeable, resulting in an additional source of flow pulsations. During each compression cycle of the pump, the piston has to move a certain distance to compress the liquid to its final delivery pressure before delivery of liquid starts. As a consequence, pulsations in the outflow occur at the pumping frequency. These pulsating flow variations are particularly disturbing to reproducibility at low flow rates because the percent magnitude of the pulsations remains substantially constant over a wide range of flow rates while the amplitudes of the peaks in the chromatogram become smaller when the flow rate is reduced, particularly when smaller separation columns are used.

The pulsations caused by the compressibility of the liquid are most often reduced by using specially designed cams that are contoured to produce the same amount of outflow of pressurized liquid at all points in each revolution of the camshaft except for a short interval at the beginning of the expulsion stroke of a piston, where the piston moves with a higher speed. This precompression phase and the resulting positive outflow pulse compensate for the compressibility of the liquid. The effectiveness of the precompression phase is dependent on a variety of parameters, including volume at the top center position of the piston, stroke volume, pressure in the pump, compressibility of the liquid, stiffness of the pumping system, and closing performance of the valves. Since not all of these parameters can be precisely determined and especially since the liquid is in general unknown, a small residual pulsation in the outflow of the pump is to be expected.

Dual-piston pumps can be classified into two types: serial and parallel. In serial pumps the first and second piston chambers are connected in series. While the first piston is delivering liquid at twice the nominal flow rate, the second piston is refilled by drawing up some of the liquid from the first piston at the nominal flow rate. The net outflow, therefore, is just the set flow rate. During the intake phase of the first piston, the second piston delivers liquid to the system at the nominal flow rate and the first piston draws in liquid from the bottle at twice the nominal flow rate. Thus the second piston, running at half the speed of the first, always delivers the nominal outflow. This type of pump only needs one inlet valve and one outlet valve because the second piston never pumps. It is only needed for damping the pump ripple. This type of pump is actually a single-piston pump with an active damping piston.

In parallel pumps the two piston chambers are connected in parallel. Each piston chamber has two valves, with the inlet valves both connected to the liquid bottle and both outlets connected to the remainder of the system. The two pistons are operated 180 degrees out of phase, but at the same speed, so that one piston is always delivering liquid while the other is drawing to refill the pump chamber. This type of pump needs four valves, but the inlet flow is constant over the full pump cycle.

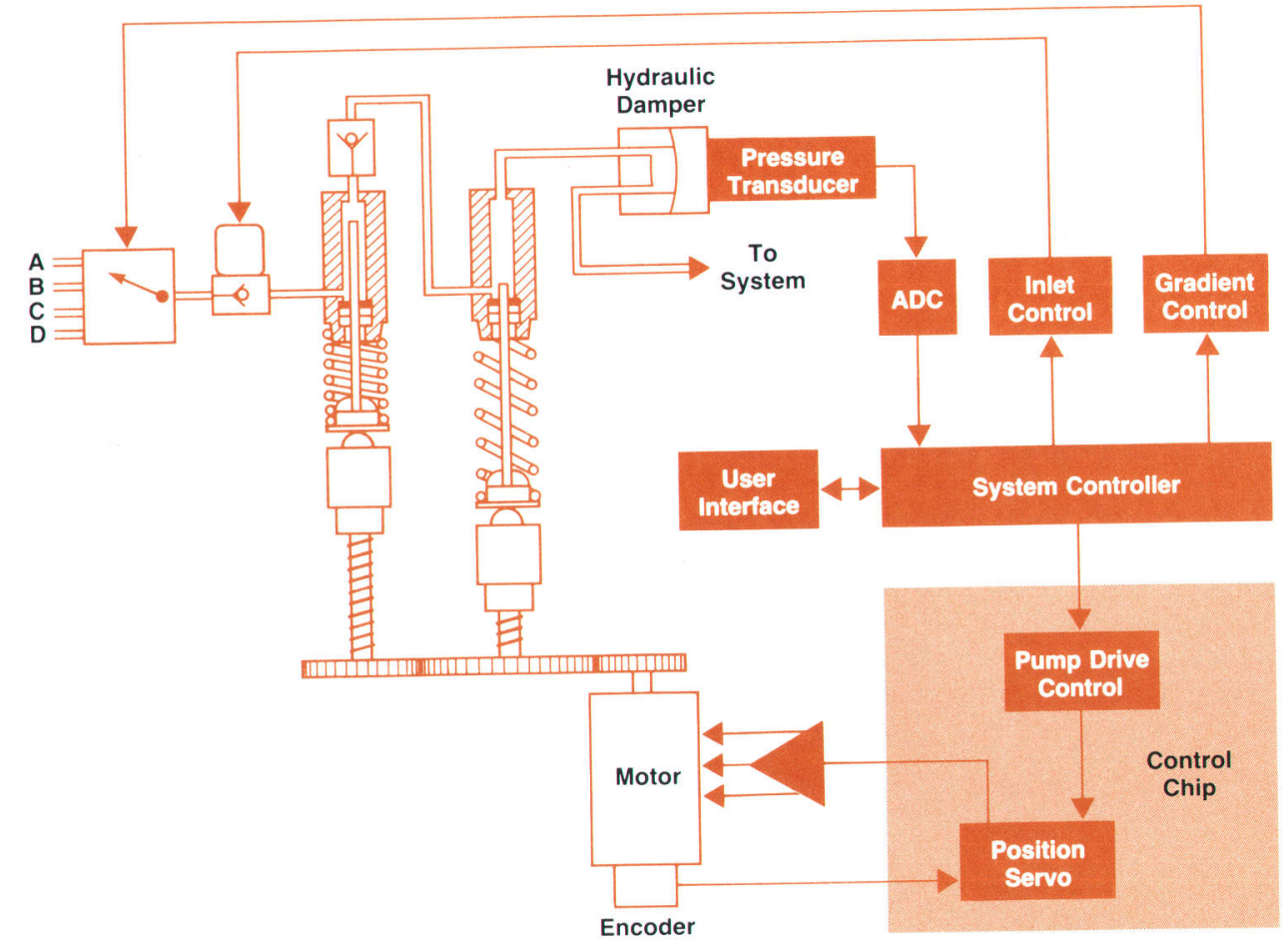

Fig. 3. Functional block diagram of the HP 1050 pump module. 
The HP 1050 pump design is a serial type because it requires only half as many valves and therefore has lower cost and higher reliability.

\section{Design Philosophy}

There is a difference in design philosophy between the earlier HP 1090 pump design ${ }^{1}$ and the current HP 1050 pump design. For the HP 1090, the approach was to use whatever hardware was needed to make the pump's performance independent of liquid properties and physical side effects. For the HP 1050, the approach is to use very simple and reliable hardware and to compensate for liquid properties and major physical side effects in other ways, based on knowledge and understanding of liquid properties and characterization of side effects.

The HP 1090 pump design splits the performance-determining elements into a metering pump, which meters a precise flow rate against low pressure, and a booster pump, which pumps the metered flow against the high system pressure. The HP 1050 pump consists only of two pistons and two valves driven by a servo motor by means of a gear and ball screw spindles. The two pistons both meter the flow and generate high pressure. Because high pressure has a big impact on metering quality, compensation is required to deliver a precise flow rate independent of solvent properties. For compensation purposes the user is required to enter a parameter for solvent compressibility.

\section{Integration Effects}

For quantitative chromatographic analysis, an integrator is used. An integrator is a reporting device that integrates the detector signal over time. Integration starts when a peak is detected and stops when the end of that peak is found. The integration result can be influenced by the pump's flow performance in several ways. The major influence is the flow rate itself. Because the detector signal is integrated over time, the same peak area may represent different component amounts. The correct relation is found by comparison with standards. However, while this can compensate for the average value of the flow rate, the pump's flow ripple will still be reflected in the reproducibility of the area results. If the detector signal is flow or pressure sensitive, this will add to the flow variation. If the pump ripple is found on the detector's baseline, the beginning and end of a peak may be misinterpreted and again the peak area will change. Integration inherently suppresses all frequencies that are high relative to the peak width well enough not to disturb the results. Therefore, a high pump ripple frequency is desirable. However, this causes the valve closing performance to have a greater influence on flow rate stability. With a fixed stroke and a wide flow rate ranging from $50 \mu \mathrm{l} / \mathrm{min}$ to $10 \mathrm{ml} / \mathrm{min}$, the pump frequency will vary by the same factor-200. A special variable-stroke function of the HP 1050 pump reduces this ripple frequency range by a factor of five. The stroke is $100 \mu \mathrm{l}$ at the highest flow rate and only $20 \mu \mathrm{l}$ at the lower end (Fig. 2). This increases the ripple frequency, especially at the lower flow rates, and thus decreases the influence of ripple on quantitative results.

Variations in the composition of the solvent mixture very strongly influence chromatographic results. The variable stroke volume leads to smaller liquid packages that can be better mixed in a given delay volume. No extra mixing is required.

\section{Pump Design}

The HP 1050 pump is a dual-piston syringe type pump with an active inlet valve and a passive outlet valve (see Fig. 3). The two pistons are connected in series. The primary piston defines the flow and the secondary piston is used for damping purposes only. Therefore, the second piston needs no valves.

Both pistons are driven by ball screw spindles. These are connected to a gear system that drives the spindles 180 degrees out of phase and the second piston at half the speed of the primary piston. This requires a drive motor that is able to reverse rotation within a very short time. The digital servo system has a high bandwidth of about $200 \mathrm{~Hz}$ and the motor shaft can be positioned with a resolution as low as one quarter of a degree. The result is a very linear drive for the piston displacement with the high reliability of a ball bearing.

The liquid is drawn in through the opened active inlet valve into the primary pump head when the primary piston is moved down. In the isocratic version the liquid comes directly from the solvent reservoir, while in the optional

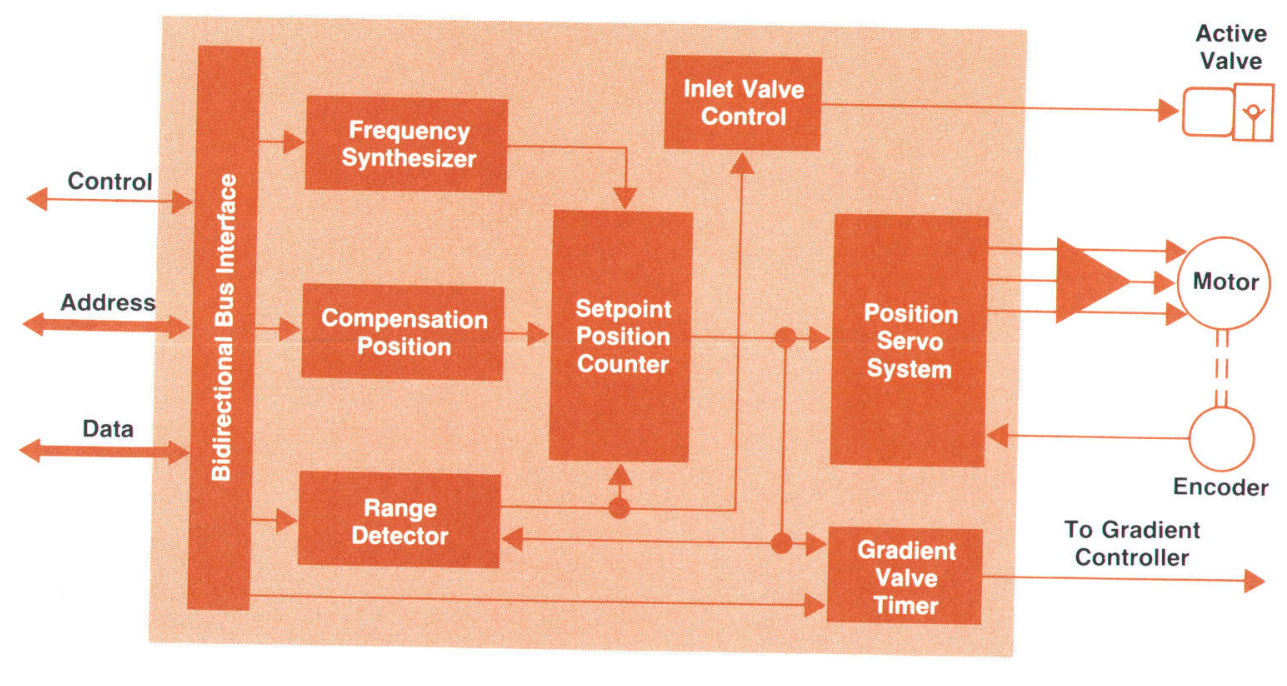

Fig. 4. Simplified block diagram of the pump control chip. 
quaternary version the multichannel gradient valve (MCGV) selects one of four solvent reservoirs at a time. When the primary piston reaches the end of the selected stroke, the direction of movement is reversed, the active inlet valve is closed, and the liquid is pressed through the outlet valve towards the secondary pump head. There are no valves at the secondary pump head because it is for damping purposes only. On the way to the chromatographic system the liquid passes the hydraulic damper, which has the additional function of measuring the system pressure.

The primary piston runs at a displacement speed that is twice the flow rate because it only draws in liquid during half of the pump cycle and delivers liquid during the other half of that cycle. While the primary piston is delivering twice the flow rate, the secondary piston, running backwards, draws off half of that volume. Thus the remaining outflow is the nominal flow rate. During the intake phase of the primary piston, the secondary piston displaces liquid, keeping up the flow rate until the first piston chamber is filled again.

The flow rate quality of such a pump relies on the switching characteristics of the valves. Therefore, an active inlet valve was chosen. A special bipolar drive allows this valve to operate in milliseconds. The pump control chip switches the valve with a delay as low as one microsecond.

For the quaternary pump, in which two or more solvents can be mixed in varying proportions to form gradients, the specially designed flowstream through the pump head allows very precise gradient formation because the flow direction of the proportioned solvent volumes does not change on the way to the system. The solvent reaches the pump chamber near the piston seal and leaves at the top of the pump head. Very good mixing of multiple solvents is achieved by the double active mixing characteristics of the two pistons in series. During the intake phase the solvent flows through the ring gap around the first piston. This forms turbulence at the top of the piston that mixes the solvent in the right order, the old composition at the top and the new composition at the bottom. This order results in smooth transitions during programmed gradients. During the delivery phase the first piston delivers the premixed liquid in the proper order while the second piston does the mixing. The ring gap and turbulence behave in the same way as for the first piston, but the main mixing mechanism is that the outflow half of the liquid passes the

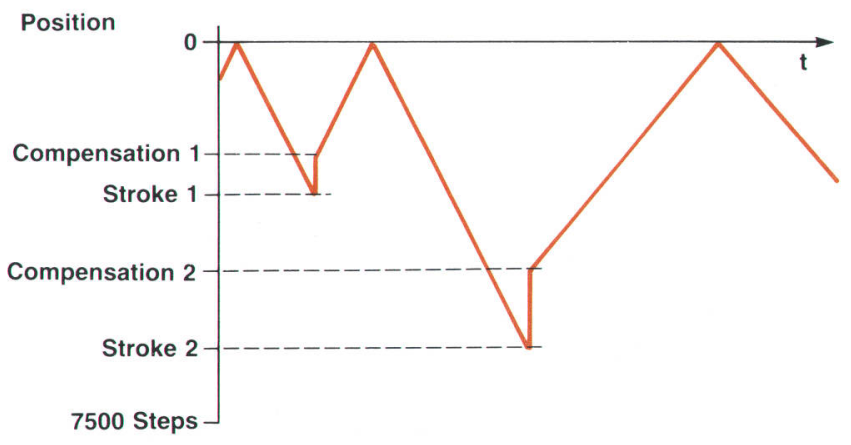

Fig. 5. Typical plot of motor position as a function of time. half that is withdrawn by the second piston. This works almost like integrating a noise signal over exactly one period. Because this mixing is inherently synchronized with the proportioning of the solvent volumes, no additional mixing device is needed to reach a composition stability of $\pm 0.25 \%$, even though the composition is formed by low-pressure proportioning.

\section{Drive Motor Position Control}

The HP 1050 pump is driven by a position-controlled motor. The motor, encoder, and digital servo technique are almost the same as in the HP 1090 pump, ${ }^{1}$ but the pump drive control is different. Early prototypes showed that it is essential for the flow performance and especially for the gradient composition that the motor control system have fast response and fine time resolution. Following the design philosophy, the influence of solvent properties is compensated by control means. Where the HP 1090 design only required a constant step frequency, the HP 1050 also needs a precise upper dead volume and a variable but reproducible stroke volume. The increased flow range of up to 10 $\mathrm{ml} / \mathrm{min}$ adds a factor of two in dynamic range, so the control response is required to be faster than $40 \mu \mathrm{s}$.

The low pressure gradient requires that the solvent selection valve be controlled so that the volumes of the various portions of solvent are reproducible. Because of the compensation, the flow rate is multiplied by correction factors. Therefore, with fixed valve timing a given portion's volume will change. With position dependent valve switching, the portion's volume is independent of the speed of the motor. 25 -nl reproducibility in volume requires $80-\mu$ s reaction time in position. The valves are not switched that fast (switching time is 1 to $2 \mathrm{~ms}$ ); however, the reproducibility of the valve switching points is better than $100 \mu \mathrm{s}$.

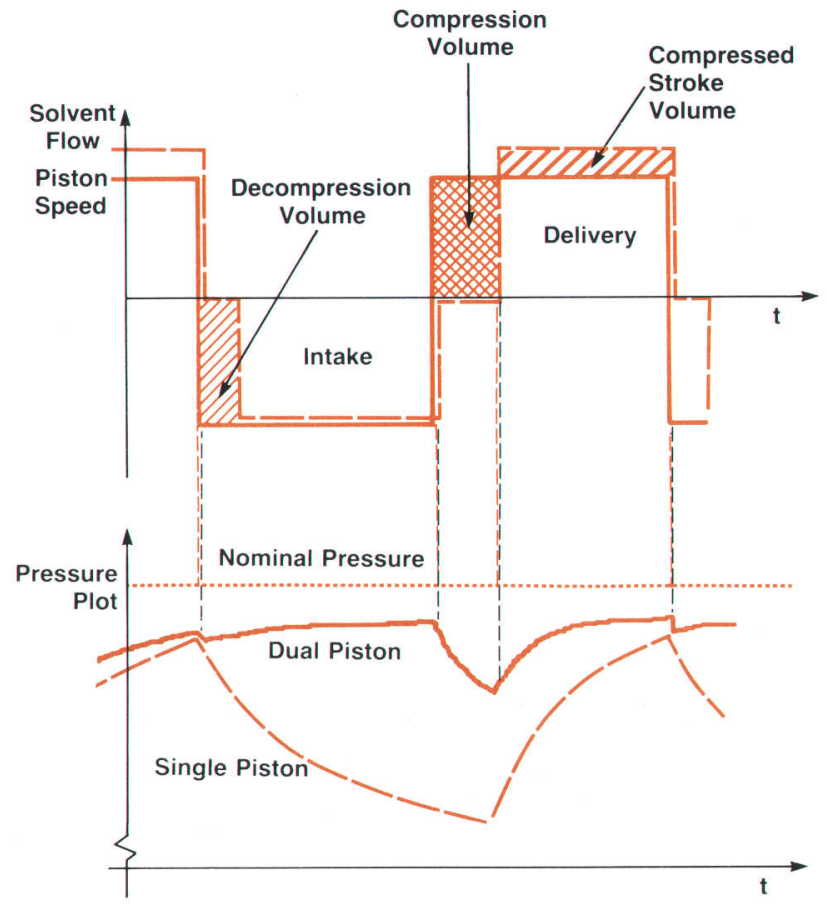

Fig. 6. Influence of solvent compressibility and elasticity. 
The pump control system performs the following functions:

- Servo position control for the motor

- Step frequency generation (15-bit and 1-Hz resolution)

a Range detection and direction control at the ends of the stroke

- Position jump function to a selectable compensation position at the beginning of the compression phase

- Synchronized control of the active inlet valve

- Position dependent generation of valve timing signals

- Bidirectional bus interface to the microprocessor.

All these functions are implemented in a single application-specific integrated circuit (ASIC, see box, page 30). All functions influencing flow reproducibility specifications are incorporated. The ASIC can control the pump with a constant set of parameters independent of the microprocessor. Fig. 4 is a simplified block diagram of the ASIC control chip.

The set of parameters required by the control chip consists of the motor speed, the distance between the zero position and the stroke end, the compensation position to which the position is set when the compression phase starts, and up to three different position marks for the gradient control. Fig. 5 shows a typical plot of motor position as a function of time.

Parameter changes input through the user interface or by run programming become active with the next pump cycle. The microprocessor does not influence the stability of flow, and therefore it is free to handle the user interface, the parameter set, and the compensation calculations that are required to make the outflow independent of the back pressure. New sets of parameters are loaded to the chip in synchronism with the pump cycle. When the pump starts

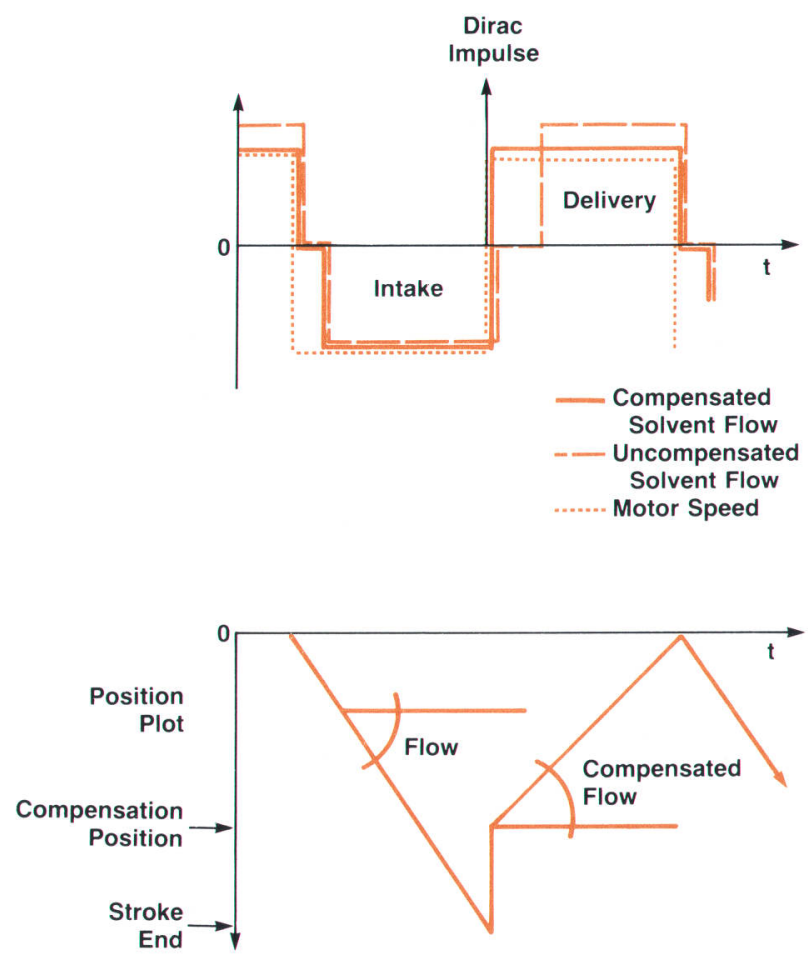

Fig. 7. Ripple compensation with compression jump. the intake cycle, the new step frequency, stroke length, and compensation position are written to the chip. When the pump starts delivery, the position marks for gradient formation are updated. This ensures that the new data is available well ahead of the time when it is needed.

\section{Solvent Properties and Physical Effects}

With regard to flow accuracy, the dual-piston serial type pump can be considered a single-piston pump with active damping (by the second piston, which has no valves). The second piston only influences the short-term flow stability. The two major effects are ripple and roll-off.

As in single-piston pumps, the internal pump head volume has to be compressed to system pressure before any liquid is delivered to the system. This compression volume (see Fig. 6) can be calculated as the sum of the elasticity of the pump head and the total volume in the pump chamber multiplied by the solvent compressibility, multiplied by the system pressure. During the time that the piston needs to travel this distance, the first-order damping characteristic of the system reduces the pressure, which changes the flow rate. This flow variance synchronized to the piston's movement is known as the flow ripple. The second piston can help reduce the ripple, as shown in Fig. 6. However, some ripple remains, its magnitude determined by the compression volume and the response of the first-order damping characteristic of the hydraulic system.

Roll-off is the reduction of outflow dependent on system back pressure. After the compression phase and during the remainder of the delivery phase the pump delivers com-

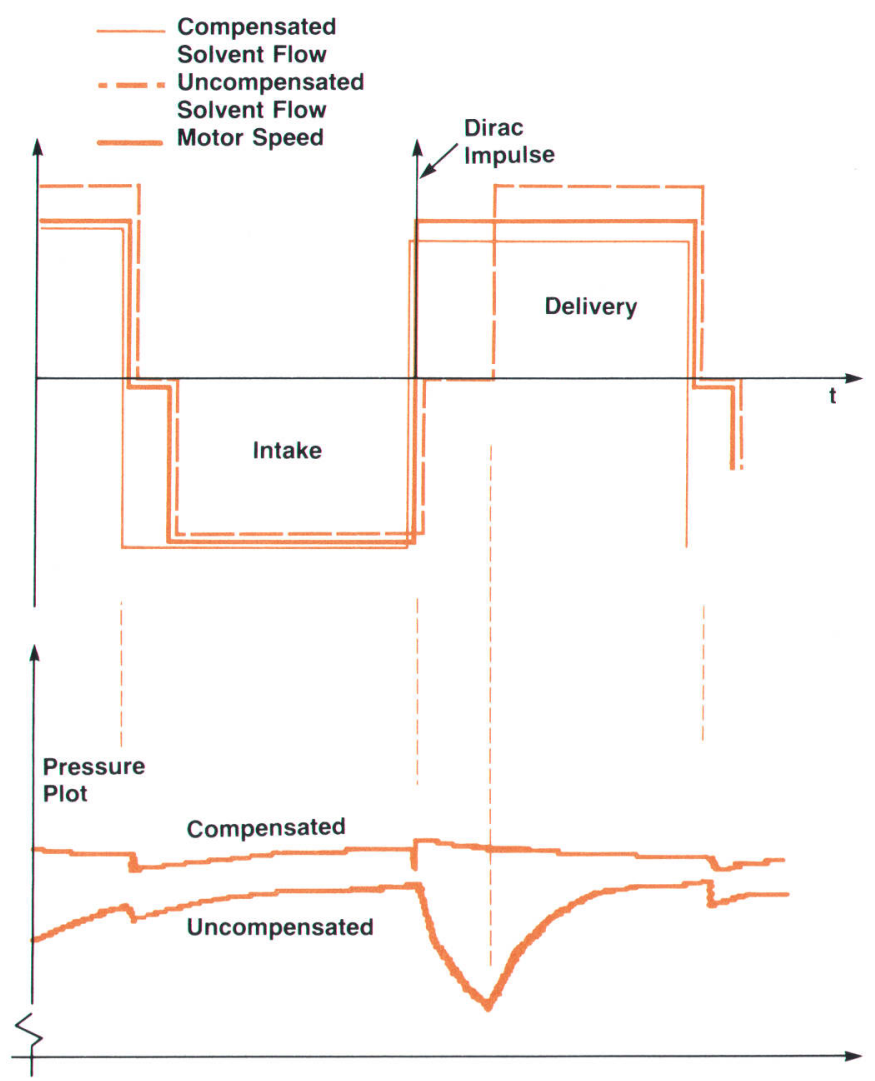

Fig. 8. Influence of ripple compensation on the pressure plot. 
pressed liquid, which expands on its way through the column. Because of the expansion, the flow delivered is higher than the volume displacement of the piston (see the compressed stroke volume in Fig. 6). At the end of the stroke the piston changes direction and the intake phase starts. Because of the pump head's dead volume, the liquid is decompressed, which means that its volume expands, and the elasticity of the pump chamber is released. During this time no new liquid is drawn in. Liquid that is not drawn in cannot be delivered. Therefore, in the long term the outflow is reduced by

$$
\mathrm{r}=\left(\mathrm{V}_{\mathrm{ot}} \kappa+\text { elasticity }\right) \times \text { pressure } \times \text { pump frequency. }
$$

where $V_{\text {ot }}$ is the dead volume of the pump and $\kappa$ is the solvent compressibility. The liquid part of this effect varies with the solvent used while the elasticity is only dependent on the mechanical construction.

To reduce the magnitude of this effect the pump's internal dead volume must be minimized. In the HP 1050 pump design, this volume is as low as $40 \mu \mathrm{l}$. This is achieved using a special absolute referencing method (explained later). Using isopropanol as a liquid, the mean compressibility is $100 \times 10^{-6}$ /bar and the decompression volume is only $4 \mathrm{nl} / \mathrm{bar}$. With such a low solvent effect, the physical elasticity is no longer negligible. Because of the plastic material used for sealing, the elasticity has a value of about $8 \mathrm{nl} / \mathrm{bar}$.

To compensate for these two negative effects, the pumpspecific ASIC chip supports a special function. Instead of moving the piston back and forth with constant speed, a Dirac impulse function having an area equal to the compression volume is added to the speed function (see Fig. 7). Theoretically, this causes a position jump, a controlled
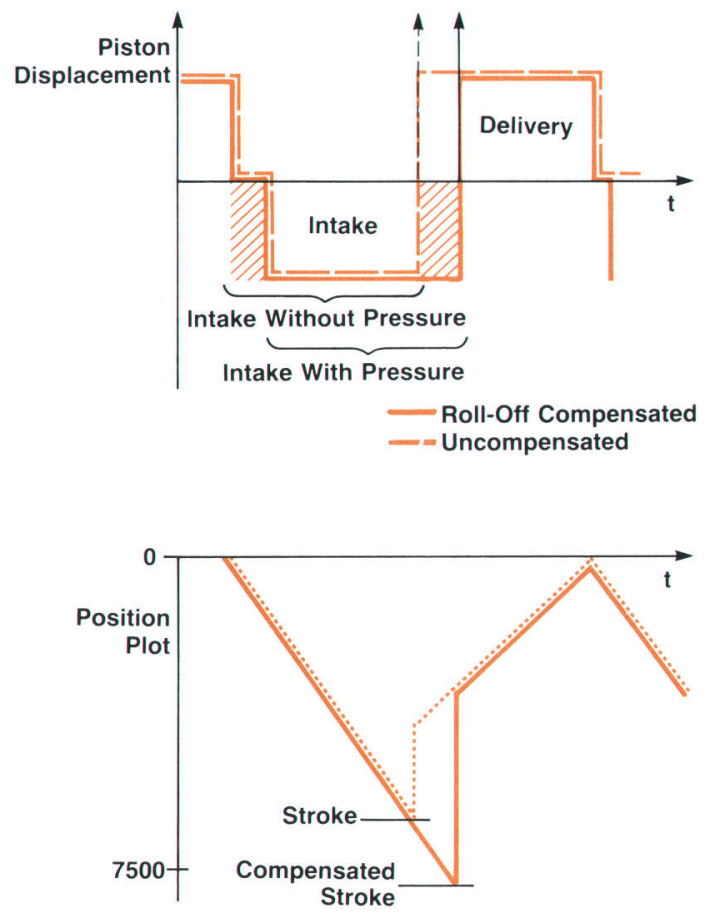

Fig. 9. Influence of lengthened stroke on intake volume. movement within zero time. In operation, the mechanics need only 40 milliseconds maximum to perform this action.

The distance between the stroke end and the compensation position is calculated by the microprocessor every pump cycle. The servo system causes the motor to perform this jump using its maximum power. The resolution is 13.5 $\mathrm{nl}$, which allows precise compensation for any stroke, pressure, and solvent.

The function just described compensates for compression volume. To compensate for compressed stroke volume, a new running speed is set. The new speed is the original speed reduced by the compression factor, so that the flow rate of the decompressed liquid at the detector remains constant, independent of system pressure (see Fig. 8). This compensated flow is set during the intake phase and is activated when the next delivery phase starts (after performing the compensation jump). These two chip functions reduce the pump's ripple to a very narrow and sharp pressure peak that is small and fast enough to have almost no influence on the chromatographic results.

To compensate for roll-off, a new function of the HP 1050 pump_-variable stroke volume-is used. The roll-off problem is that, because of the decompression phase, the pump cannot draw in as much liquid as the stroke length would indicate. The intake stroke is effectively shortened, and therefore, to compensate, the stroke has to be lengthened by the same amount. The variable stroke feature makes it easy to set the pump control chip to a stroke value that is the sum of the nominal stroke plus the decompression volume (see Fig. 9). During the time it takes to draw in this added volume, the second piston keeps the flow constant. When the ripple compensation jump is executed, the decompression volume is compressed again to the sys-

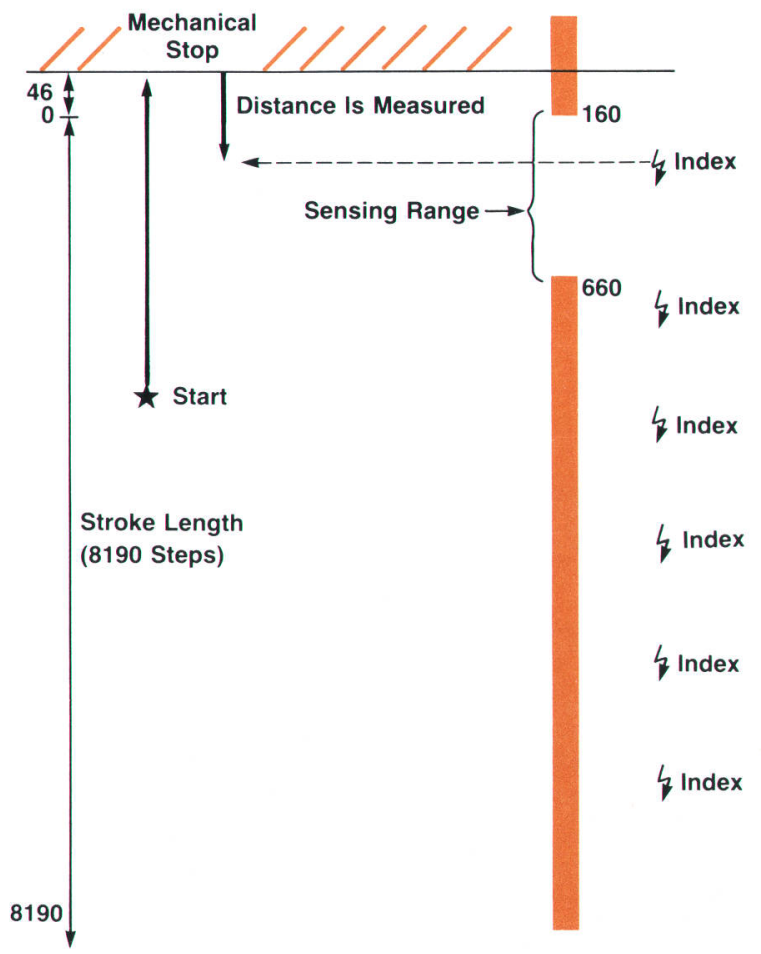

Fig. 10. Determination of an absolute reference position. 


\section{Pump Control Chip}

The design, mechanical construction, and compensation functions of the HP 1050 pump module require a high-performance motor and control system with high resolution, dynamic range, and power.

Dynamic performance can be divided into two parts. One is the ability to accelerate and decelerate, which is mainly determined by the torque and internal inertia of the motor. The other is the useful output torque as a function of frequency. Dc motors have an advantage in this area, while steppers, especially without feedback, are weak here. Steppers tend to show resonances at various frequencies, and reversing the current in their inductive windings with a limited voltage takes time. The HP 1050 pump would require a stepper motor running at a $125-\mathrm{kHz}$ step frequency with more than 1000 steps per revolution. Even with no load and a long rampup time, this is difficult to achieve. Standard dc motors are not allowed in analytical laboratory instrumentation because of sparks and the risk of explosion. Stepper motors generally have good resolution or dynamic range but not both, and are difficult to use with variable loads.

To meet the requirements it was decided to use a position control servo system of a type used in many HP applications including robotics, plotters, and the metering pump in the HP 1090 HPLC system, predecessor of the HP 1050 Series. ${ }^{1}$ For the high-dynamic-range requirements, it was found that a variablereluctance (VR) motor, which is driven with control circuitry like a brushless dc motor, provides the best performance.

The HP 1050 pump design requires high power to pump liquid against high pressure. The running speed is selectable from 0 to $10 \mathrm{ml} / \mathrm{min}$ with a resolution of $1 \mu \mathrm{l} / \mathrm{min}$. This operating dynamic range is increased by the compensation calculations, so the internal resolution is $0.4 \mu \mathrm{l} / \mathrm{min}$ for a $1-\mathrm{Hz}$ stepping frequency. This implies a maximum stepping frequency under a 200-bar load of $25 \mathrm{kHz}$, or $12.5 \mathrm{kHz}$ with a 400 -bar load. The VR motor meets these requirements. It also has enough power to accelerate the pump from zero speed and jump 1000 steps within $40 \mathrm{~ms}$.

The servo system is all digital. This makes it easy to integrate into an ASIC (application-specific integrated circuit) chip, which saves cost and increases reliability. There were servo chips available, including the position control chip designed for the HP 1090 metering pump, but these would have needed companion microprocessors running with at least a $1-\mathrm{kHz}$ interrupt rate to achieve the pumping performance. Therefore, it was decided to design a new standard-cell chip that incorporates all the required control functions to operate the pump hardware. An external microprocessor is only needed for parameter setting and the compensation calculations with a timing requirement of $200 \mathrm{~ms}$. These can be done by the system's main processor and no extra chip is required.

\section{Chip Design}

Our experience with standard cell design was very helpful. We worked together with HP's Cupertino Integrated Circuits Division, and the first design was functional. No changes were needed. When we started the chip design, HP technology had just changed from $\mathrm{CMOS}-\mathrm{H}$ to $\mathrm{CMOS} 40$, and the design station from HP EGS to HP EDS. Since all our experience was on the EGS system and the old HP 1090 chip design was to be a third of the new HP 1050 chip, it was decided to design for CMOS-H on EGS and convert the design to CMOS40.

Fig 1 shows a functional block diagram of the pump control chip. The heart of the chip is a 14-bit position servo system that is designed to drive a special VR motor developed by HP Laboratories in 1984 and manufactured by Warner. The built-in commutator is fixed for a three-phase motor and an encoder that has a factor-of-60 higher step count than the motor steps (for example, a 3-phase VR motor with 24 steps/rev and a 360-slit encoder with 1440 steps/rev).

The setpoint position counter can be accessed directly via the interface bus by writing to it through the compensation position register in position control mode. The motor will perform a jump to the written position.

The chip contains circuitry that makes the servo frequency

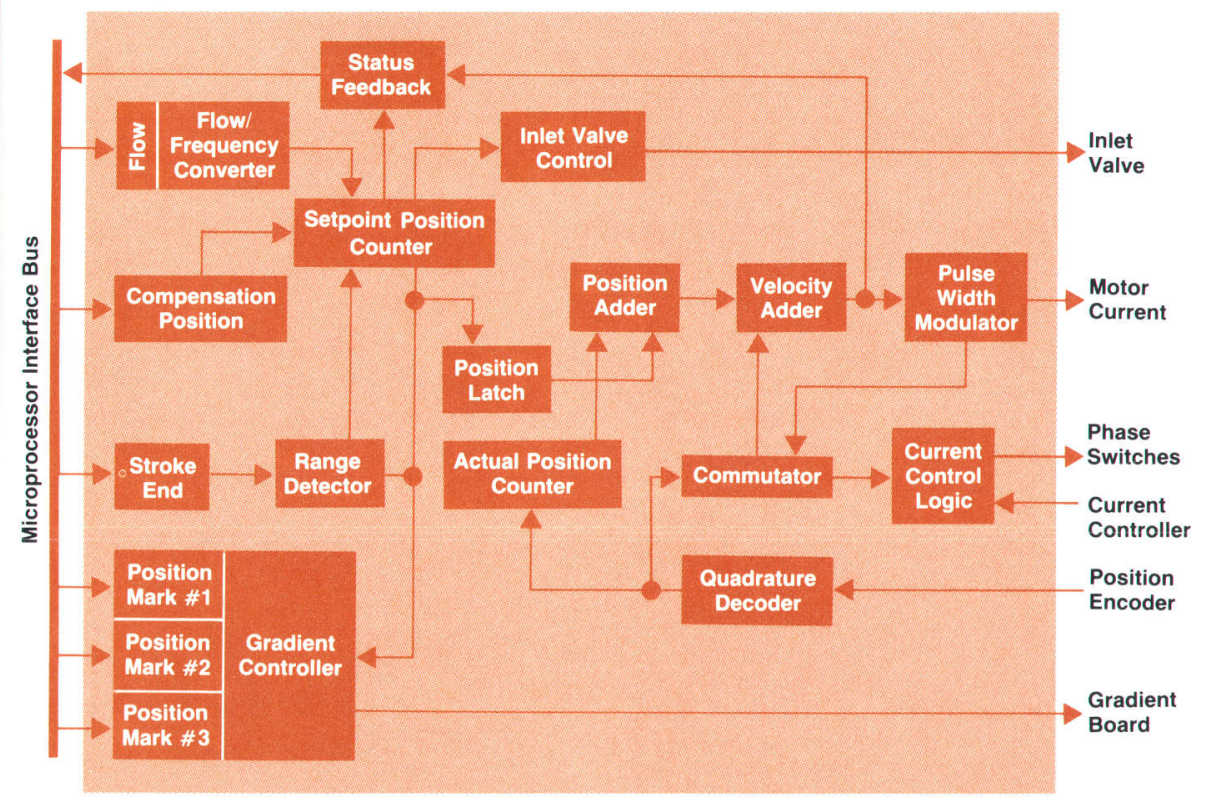

Fig. 1. Functional block diagram of the HP 1050 pump control chip. 
controllable, so the motor speed can be the controlled parameter rather than position. This is the main mode of operation when driving a pump. The motor speed is settable in a wide 15-bit range with $1-\mathrm{Hz}$ resolution. A control signal applied to one of the chip's pins causes the frequency range to be scaled up by a factor of 16. The motor direction is another control bit, so the running speed is selectable over a full 16 -bit range $( \pm 32,767$ counts).

Two position markers can be set with 14-bit range. One is the stroke end and the other is the compensation position. When the motor's setpoint position reaches the stroke end, the motor direction is reversed. At the zero position, the direction is again reversed, giving the back-and-forth pumping action. When the compensation position is set to a number smaller than the stroke end, the motor will jump to that position before it starts moving backwards. This function is used for flow compensation.

Three more registers allow the setting of position marks for the motor. When a mark is reached the chip sends out a pulse.
These pulses are used in the pump to switch the gradient mixing valves synchronously with the pump's sucking action.

Some control bits round out the functions of the chip so that it can be used for universal motion control, specialized for the particular motor used for the pump. The chip includes all functions needed to drive the HP 1050 pump. Once the parameters are set, the chip will ensure stable flow for days or years without any further microprocessor action. Thus the pumping performance is totally independent of processor load or interrupt priority

\section{Reference}

1. W. Geiger and H. Vollmer, "A New Solvent Delivery System," Hewlett-Packard Journal, Vol. 35, no. 4, April 1984, pp. 13-20

Fred Strohmeier Klaus Witt

Development Engineers Waldbronn Analytical Division tem pressure. The intake volume, therefore, is exactly the nominal. The pump frequency remains constant-there is only a slight phase shift of the intake phase. Since flow rate is volume times frequency, it is also exactly the nominal, and there is no roll-off.

\section{Absolute Referencing}

During normal operation the drive system of the pump works in an absolute position control mode. For this mode, it is necessary to know at least one absolute position reference. Former designs used light switches or magnetic sensors for the absolute feedback. For the HP 1050 pump, price and reliability considerations led to a new solution.

The absolute position reference has a direct influence on the primary piston's dead volume, which leads to flow disturbances like the roll-off and ripple explained earlier. The HP 1050 pump is designed to compensate for these disturbances, but it is required that the dead volume be known, constant, and as low as possible. The tolerances of regular position sensors would require complex adjustments, because the primary piston's top dead center is designed to be only $77 \mu \mathrm{m}$ away from the top of the pump head. A reference signal is available from the motor position encoder, but it occurs every revolution, 6 or 7 times during the stroke (see INDEX signal in Fig. 10). Because it occurs more than once during the stroke, this index signal cannot be used directly. It is necessary to find the first occurrence. Therefore, a special algorithm is required for absolute referencing.

For this algorithm, special functions are implemented in the motor controller chip. The motor current can be read, the encoder index signal is visible to the microprocessor, and in a certain position range the setpoint position counter can be loaded with a predefined offset value. During assembly, the pump mechanical gear system is adjusted so that the first encoder index signal occurs within about 160 to 660 encoder steps away from top dead center. To find the absolute reference position, the primary piston is moved slowly towards the pump head while the microprocessor monitors the motor current. When the motor current, an 8-bit digital word, increases in value, the piston is at top dead center. The piston is then pulled back and the micro- processor measures the exact distance moved until the first index pulse occurs. This distance is used to calculate the numeric value to which the setpoint position counter must be loaded each time the piston reaches the index pulse that occurs inside the sensing range (see Fig. 10). Subsequently, the servo always reverses direction when the setpoint counter reaches a count of zero. Thus, for example, when the first index pulse is found 246 steps away from the top, the setpoint counter is loaded with 200 to ensure

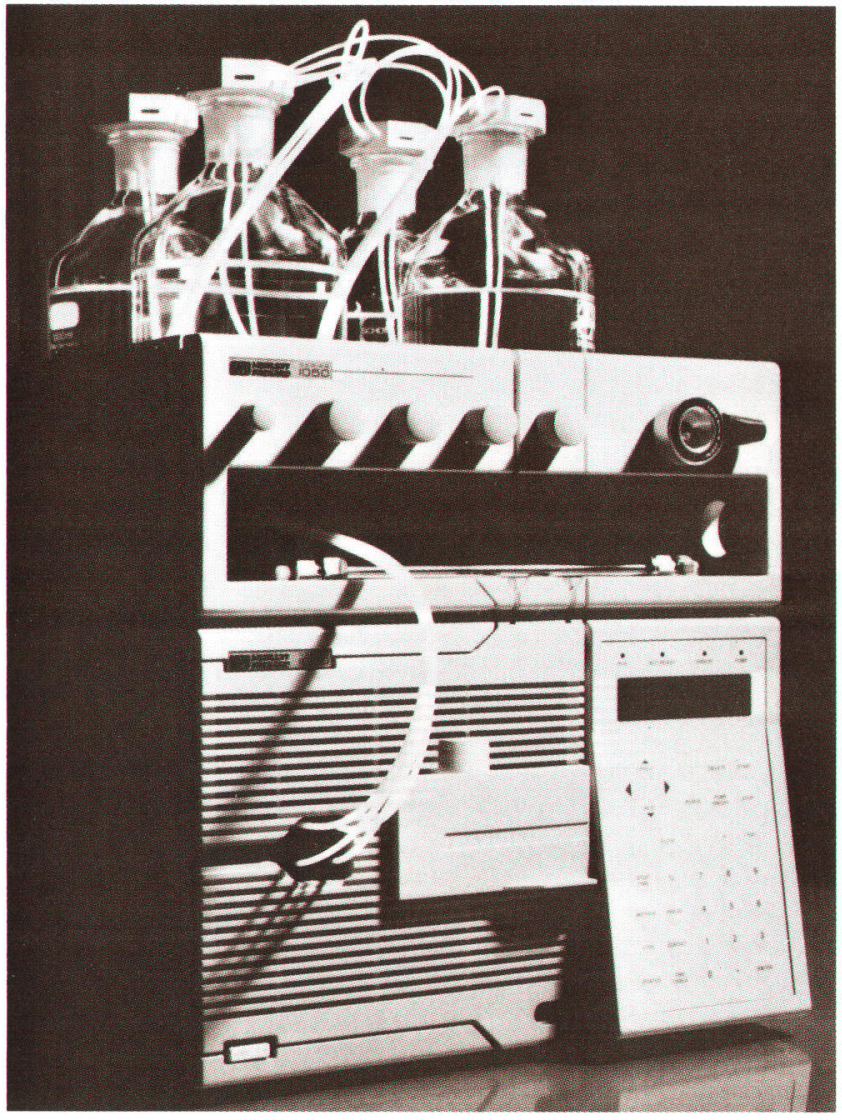

Fig. 11. The quaternary HP 1050 pump module can mix up to four solvents to form gradients. 
that the piston reverses direction exactly 46 steps before top dead center. The referencing algorithm is accurate within four steps, which is $53 \mathrm{nl}$ in dead volume or 6.7 $\mu \mathrm{m}$ in piston position.

When the pump head is changed to one with different tolerances, it is not necessary to readjust the position sensor. The referencing algorithm aligns itself in a wide range of $0.8 \mathrm{~mm}$.

This initialization (computation of the numeric value) is performed whenever the pump motor is started. The position referencing (loading of the numeric value into the setpoint counter) is performed every time the piston approaches the top. Therefore, disturbances on the encoder lines are not accumulated, and long-term operation is not a problem.

\section{Gradient Formation}

Most chromatographic analysis requires solvent blending. At least during method optimization, it is useful to have a free choice of solvent composition. Some applications require the solvent composition to be modified during the analysis. This mode is called gradient programming.

The HP 1050 pump design is a low-pressure gradient system, which means that the solvent mixture is formed ahead of the pumping apparatus. The mixture is formed by sequential proportioning of the different liquids during the intake phase of the pump. Because some liquid combinations as a mixture can solute less gas than the separate liquids, the gas content has to be lowered below the saturation limit. This is done by purging the solvents with helium. It has been found that helium is able to replace the gas in a liquid such that the final amount of helium in the liquid is much lower than the original amount of gas. Some detection systems do not allow oxygen in the mobile phase but helium is well accepted. The HP 1050 quaternary pump (Fig. 11) is shipped with a solvent preparation cabinet that

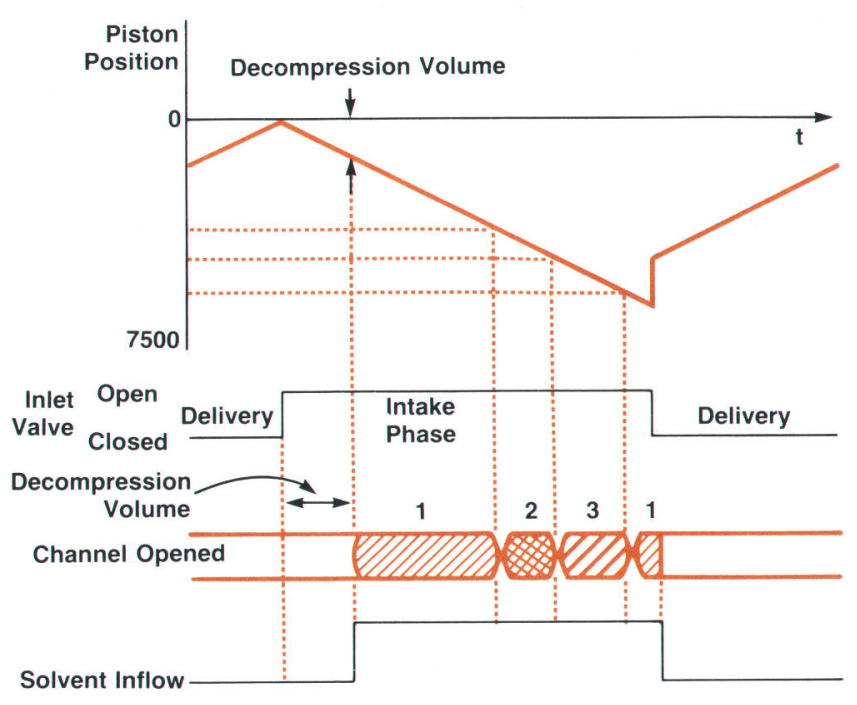

Fig. 13. Position dependent solvent proportioning.

includes solvent bottles, bottle caps with solvent filters, and a set of helium flow control valves. As an option, the solvent cabinet can have a manual injection valve, and there is a column compartment that holds the analytical column and a leak tray to collect leaking solvent.

A dual-piston serial type pump draws liquid during only half of the pump cycle. This increases the switching requirements for the proportioning valve. The number of different portions also affects these requirements because, as the number of portions increases, the probability that some of the percentages are low also increases. At a flow rate of $10 \mathrm{ml} / \mathrm{min}$ and a stroke of $100 \mu \mathrm{l}$, the switch-on time for one channel is as low as three milliseconds per percent in composition. To achieve the specified $\pm 0.25 \%$ absolute composition precision, the valve is required to
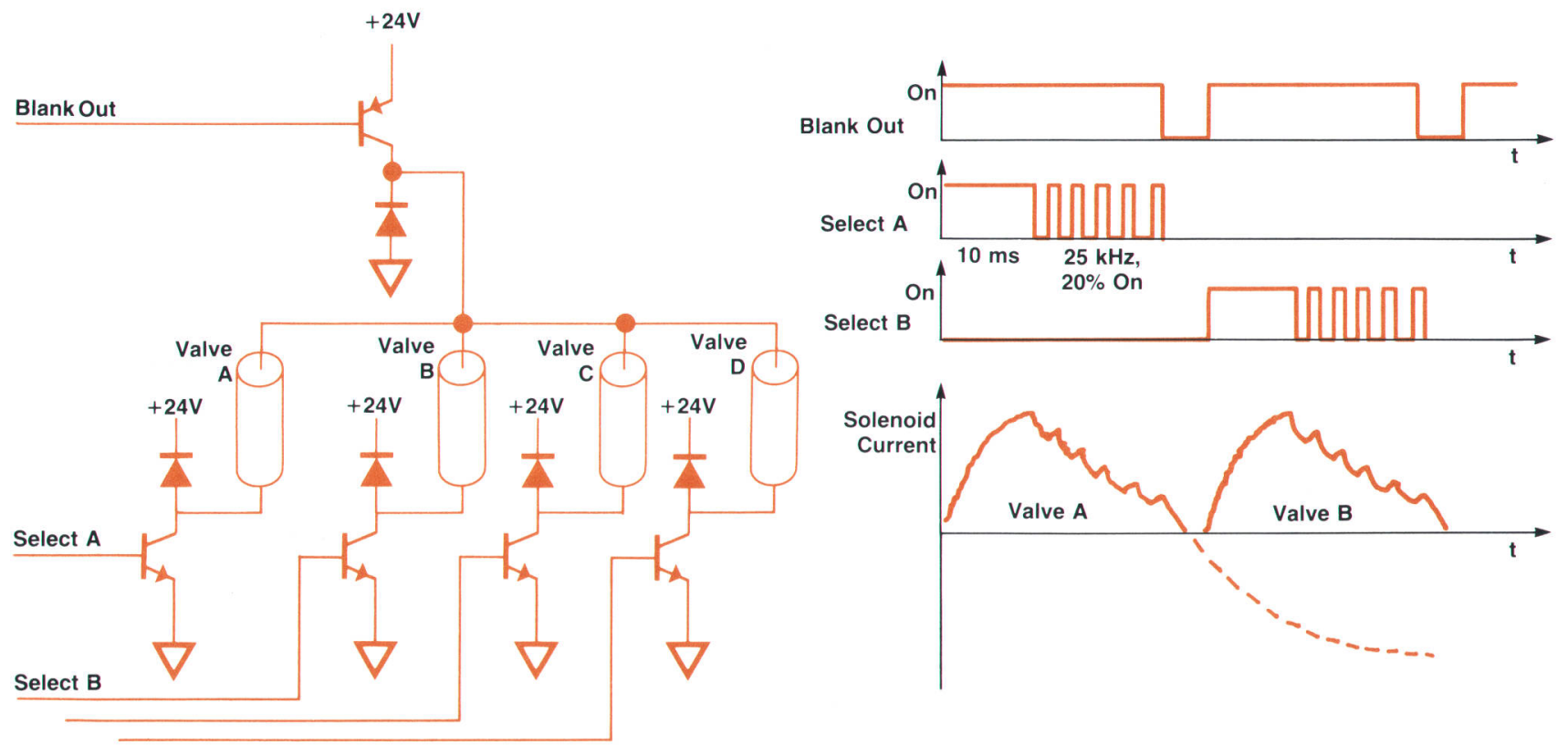

Fig. 12. Schematic diagram and waveforms for the inlet valve of the quaternary pump. The waveforms indicate transistor states, not voltage levels. 
switch within $1 \mathrm{~ms}$.

To achieve such fast switching with magnetic solenoids, the overdrive technique is used. For on times up to $10 \mathrm{~ms}$, the valve is driven by a voltage five times nominal. For longer on times, the voltage supply is chopped after $10 \mathrm{~ms}$ at a rate of $25 \mathrm{kHz}$ and the duty cycle is $20 \%$. When a changeover from one channel to the next occurs, at first all channels are switched off to avoid crossflow between the different solvent containers. This "all channels off" situation allows all four solenoid drivers to be combined so that only five power transistors are needed (see Fig. 12).

If there is a small solvent proportion right at the beginning of an intake phase or right at the end, there is a chance that the proportion will be influenced by the switching of the inlet valve or by the decompression phase, which prevents the drawing of solvent for a certain time. This effect can be large enough that no solvent will be drawn for that portion, thereby spoiling the composition accuracy. To prevent this, a function called the primary channel is implemented. The primary channel is the one that is opened at the beginning of the intake stroke, and therefore is the one that is influenced by the pressure dependent decompression phase. It is selected by an automatic function or by the user. This primary portion is split, and is drawn in partly at the beginning and partly at the end of the intake stroke (see Fig. 13). Up to two smaller portions can follow in sequence between the two parts of the primary portion. Because the same channel is opened both times the inlet valve is switched, the influence on composition is automatically compensated. Any change in the decompression volume because of changing pressure reduces or enlarges the first channel's portion. By the compensation calculations described earlier, the stroke is adapted to the decompression volume, and therefore the last channel's portion is enlarged or reduced correspondingly. Since the first channel and the last are the same, the effects compensate. The same occurs when the valves have a delay between the electrical signal and the mechanical switching action. This changes the phase between the proportioning and the pump cycle, but all portions remain the same. The user is expected either to use the automatic function or to decide which channel is primary (first).

One channel is always open, even when the pump is in the delivery phase. This ensures that the pressure between the gradient valve and the inlet valve does not rise even when the inlet valve has backflow because of dirt or a malfunction.

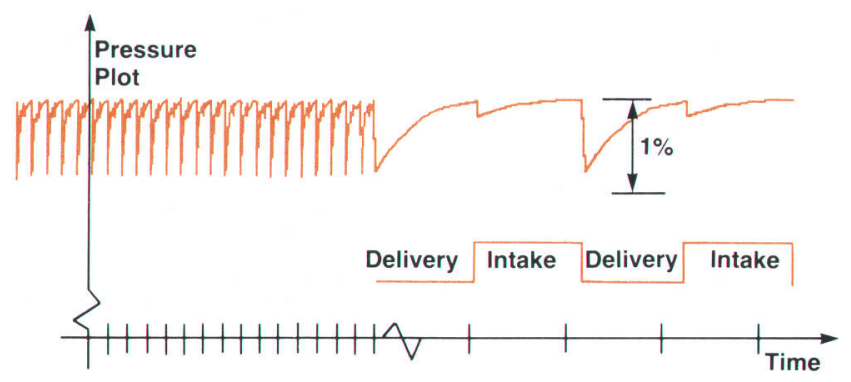

Fig. 14. A typical pressure trace and its relation to the pump cycle (right side time scale expanded).

\section{Diagnostics and Monitoring}

The HP 1050 pump is designed on the basis of a complete understanding of the effects and parameters that influence the performance of a high-pressure pump system. The system internally handles all the values that influence the performance as single and known parameters. These parameters are given as digital values to a pump control circuit that forces the precision pump mechanism to produce the expected solvent flow accurately. The simple structure of the pump mechanism and the open design of the pump control logic allow the microprocessor to compare expected events with the real situation for a variety of parameters and thus generate diagnostic information that is of real value to the user.

Faults are detected either during self-tests or when unwanted events occur. The goals are to find faulty components that need to be replaced, or to detect events that for safety reasons require that the system be switched off before it destroys others or itself. In nearly all cases, when a fault is detected the system will stop normal operation and inform the user that some action or service is needed. The operating and service manual contains suggestions for further diagnostic actions to point out the faulty element.

The system can detect 24 different error states, some of which may indicate faults. In all cases, detection of one of these states places the instrument in an error state. In some cases, a predefined error method will be called for and executed. The system will print the reason for entering the error state on the display to inform the user. The error conditions can be placed in three categories:

- Start-up errors, such as RAM test failed

- Initialization errors, such as pump reference lost

- Operating errors, such as pressure exceeds 420 bar.

There are always some effects that cause the performance of a pump system to become worse with time, eventually becoming insufficient for the application. If the instrument is able to detect these changes early enough and inform the user, the user will be able to plan for corrective action or repair. The HP 1050 pump has a number of continuous monitoring functions that watch for such effects. The results of these monitoring functions do not have a direct influence on the pump's operation. They will not cause the pump to switch off. They are placed in the internal memory "logbook" and can be accessed by the user, who can initiate further actions. There are four diagnosis levels:

= Level 0: On-line monitoring is switched off.

- Level 1: On-line monitoring results are written to the logbook and can be called for using the STATUS key. No further actions are taken by the instrument.

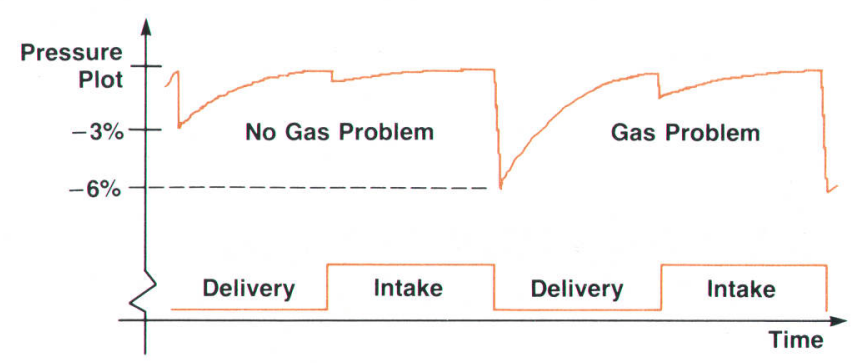

Fig. 15. Gas bubble detection using ripple information. 
- Level 2: On-line monitoring results are written to the logbook and can be called for using the STATUS key. The system goes to the not ready state. The N'READY LED is lit and the N'READY control line of the remote control bus is pulled low to prevent further injections.

- Level 3: For use only by production or service personnel, mainly to measure the available stroke to check the pump drive adjustment.

\section{On-Line Pressure Monitoring}

In normal operation, the system pressure is continuously monitored and displayed in selectable pressure units (MPa, bar, or psi). There is an analog output at the rear of the instrument for plotting the pressure on an integrator, which can be the same one that is used to monitor the detector output.

A major reason for monitoring the pressure is safety. The user can type in upper and lower limits for the pressure. The upper limit is used to protect the column against too high a pressure, while the lower limit is used to detect a dramatic leak in the system or that the solvent reservoir is empty and the pump is drawing air.

This absolute pressure monitoring does not require precise time resolution. However, short-term pressure variations are also monitored for such purposes as gas bubble detection and flow symmetry analysis. So as not to overload the microprocessor with complex calculations at a high speed, a specially designed analog-to-digital converter (ADC) is built into the pump. Called the relative ADC, it delivers a data word proportional to the relative difference of the input voltage and a reference. Absolute values are calculated from this relative data about once per second, while the relative information is available without calculation about every $10 \mathrm{~ms}$.

\section{Ripple Measurement and Gas Bubble Detection}

In a normal HPLC system, the hydraulic resistance is linear, and therefore the pressure signal is a good tool for monitoring the flow stability. However, the absolute value of the resistance depends on solvent properties and temperature, as well as variations in the flow path, so the absolute value of the pressure cannot be used. The relative information, on the other hand, is very useful for monitoring functions.

Fig. 14 shows a typical plot of HP 1050 pump pressure. Most of the short-term variation is synchronous with the pumping action. This peak-to-peak variation is the ripple.

In the HP 1050 pump, ripple compensation calls for a

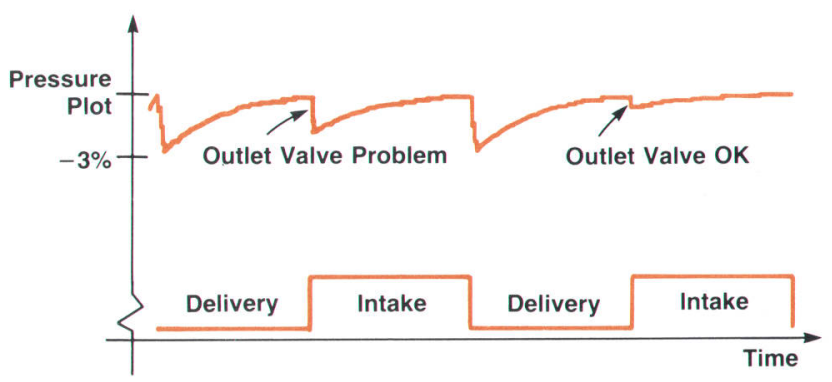

Fig. 16. Improper closing of the outlet valve leads to secondary ripple. high-speed precompression phase right after the primary piston begins to deliver. This phase is less than $35 \mathrm{~ms}$ in duration. Because this action will not always perfectly compensate for the solvent's compressibility, the system pressure after the compression phase will vary. The HP 1050 pump exhibits a positive ripple if the pressure after the compression phase is higher than before and a negative value otherwise. A peak-to-peak value normally is positive only, but the extra sign indicates whether the pump is overcompensated or undercompensated.

With a gas bubble in the primary cylinder, the compressibility of the volume will increase dramatically (see Fig. 15). Therefore, the mismatch between the ripple compensation and the actual ripple can give information about the gas content. To detect gas bubbles, the last pressure value before the pump changes to the delivery phase is stored. This value is compared with a pressure value measured about $40 \mathrm{~ms}$ after the direction change. Because all pressure values are available as percentages of the mean value, the ripple can be calculated as simply the difference between these two values. If the ripple is greater than a limit that depends on the compressibility, a gas bubble is suspected and a message is stored in the logbook.

If the bubble is an isolated phenomenon, that is, if gas is not entering the pump head continuously, then after some time the cylinder will be filled completely with solvent and a message will be entered into the logbook indicating that the gas problem has been solved.

The secondary ripple, that is, the pressure drop when the second piston starts delivery, is used to check the closing function of the outlet valve. This ripple is normally well below $1 \%$. If the valve is closed by backflowing liquid, this secondary ripple is increased, as shown in Fig. 16, and a message is generated.

\section{Flow Symmetry Analysis}

Continuous backflow through the outlet valve shows a different pressure shape and is detected using flow symmetry analysis. Flow symmetry analysis is a monitor function that derives the end value of the pressure under the assumption that each piston will deliver the same flow forever. Essentially, this is the value the pressure approaches asymptotically during the time a given piston is delivering. The values are calculated for each piston sepa-

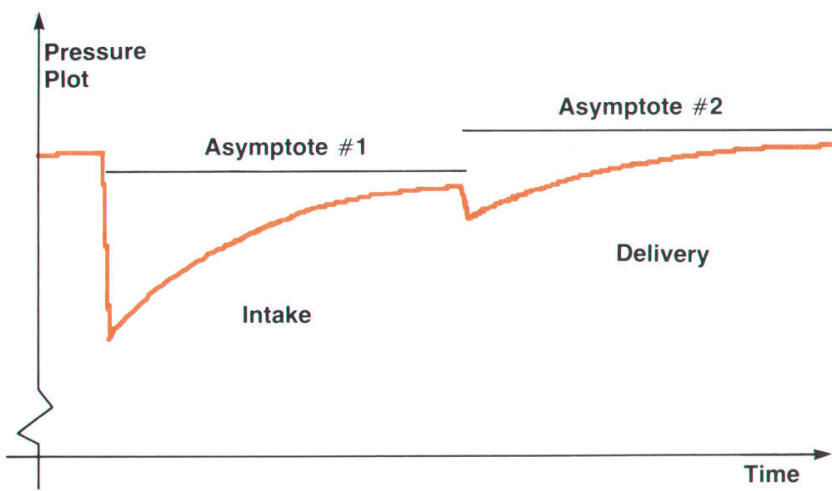

Fig. 17. An example of flow asymmetry caused by a leaky first piston 


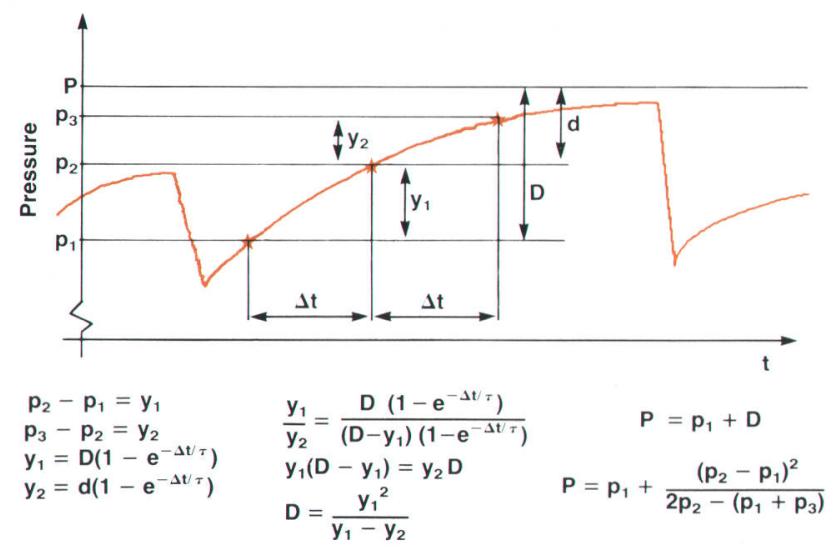

Fig. 18. Calculation of the pressure asymptote without knowing the damping function.

rately and are called asymptote \#1 and asymptote \#2. Asymptote \#1 is calculated from the pressure trace of the primary piston and asymptote \#2 from the pressure trace of the secondary piston.

An optimal pump system will show pressure differences between the two phases of the pump cycle if the mean values or the final values of each cycle are compared. This is because only the primary piston compresses the solvent to system pressure. However, the asymptotes of both phases should be identical under stable conditions. If these values differ by more than 1\% (Fig. 17), a logbook message is generated indicating either that the primary piston leaks or that there is outlet valve backflow, depending on which asymptote is greater. Trends, such as pressure slopes, are filtered out using the values from the last cycle.

For flow symmetry analysis, the microprocessor has to calculate the asymptote of an exponential function twice per pump cycle. To reduce the calculation effort, the data points are selected so that three equally spaced points are spread over each half pump cycle. This avoids the need to calculate an exponential function of an unknown firstorder damping system (see Fig. 18).

\section{Summary}

The HP 1050 pump module is a very simple but precise mechanism driven by a high-dynamic-range servo drive. The design merges reliable known technology with powerful new control capabilities. An active inlet valve, custom IC technology for the position servo, and a complex control algorithm running on an MC68008 microprocessor result in a pump that performs well, is easy to operate and maintain, and offers automation features to address a variety of applications.

\section{Acknowledgments}

Completion of this project was made possible by the motivation, knowledge, and teamwork of many people, especially the R\&D team. At this point we want to thank Manfred Berndt who worked on the pump mechanics, Hans-Georg Weissgerber who designed the valves, the instruments cabinet, and some plastic parts, Hans-Georg Haertl who worked on the solvent cabinet and the gradient valve, Manfred Koelmel who designed the custom IC and the pump-specific electronics, Klaus Weber who helped with the programming, Werner Schneider who helped with designing some plastic parts, Manfred Seitz as production engineer, and all others in the various departments whose work was helpful in finishing this project. We would also like to thank all the people in HP's Circuit Technology Group who contributed advice, motivation, and technical understanding to make the chip function on the first shot.

\section{Reference}

1. W. Geiger and H. Vollmer, "A New Solvent Delivery System," Hewlett-Packard Journal, Vol. 35, no. 4, April 1984, pp. 13-20. 


\title{
A New Generation of LC Absorbance Detectors
}

\author{
Two absorbance detectors are available for the HP 1050 \\ Series modular LC system: a high-sensitivity programmable \\ scanning detector and a high-speed, multiple wavelength \\ diode array detector.
}

by Axel Wiese, Konrad Teitz, Volker Brombacher, Günter Höschele, and Hubert Kuderer

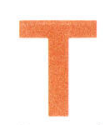
HE MOST IMPORTANT DETECTION PRINCIPLE in liquid chromatography (LC) is absorbance detection in the ultraviolet and visible wavelength ranges. Sample molecules with chromophore groups absorb light and are excited into higher electronic energy levels. The observed spectrum, a plot of light absorbed as a function of wavelength, is characteristic for any species and is therefore helpful in identifying compounds previously separated in a liquid chromatography column.

There are two types of LC absorbance detectors, each having its own special features and characteristics. The first is the forward optics detector. White light is separated by a wavelength dispersive element_-typically a gratingand one selected wavelength passes through the sample in a flow cell of an LC system. This type of detector is set to one wavelength at a time. Spectra can only be acquired by stopping the pump flow and turning the grating from one position to the next to change the wavelength in the cell. In the early 1980s, HP introduced the reverse optics detector. White light first passes through the flow cell, where it is attenuated at wavelengths characteristic of the sample molecules. It is then separated and directed by a grating towards a diode array. Each diode detects light in a very narrow wavelength range, and there are enough diodes that all wavelengths of interest are detected simultaneously. Originally used for pure research, diode array techniques are beginning to be used for routine analysis in quality control laboratories. An invention of the 1970s, the holographically fabricated concave grating, can resolve the wavelength range on a nearly linear focal curve. This kind of self-scanning photodiode-array detector can produce dozens of spectra in a second, on-line. With a microcomputer handling the resulting data stream, a speed unattainable with forward optics detectors is achieved in the measurement of separated compounds. For the HP 1050 Series Liquid Chromatographs, the forward optics technique is now driven to its highest sensitivity so far with the HP 79853A Variable Wavelength Detector (VWD), while the HP 79854A Multiple Wavelength Detector (MWD), the reverse optics solution, features a wide range of spectral capabilities.

\section{Theoretical Background}

The basic equation for absorbance spectrometry is the Lambert-Beer law:

$$
A(\lambda)=\epsilon(\lambda) C D=\log \frac{I_{o}(\lambda)}{I(\lambda)}
$$

where $A(\lambda)=$ absorbance in absorbance units (AU) as a function of wavelength

$\epsilon(\lambda)=$ extinction or molar absorption coefficient as a function of wavelength

$\mathrm{C}=$ molar solute concentration

$\mathrm{D}=$ path length $(\mathrm{mm})$

$\mathrm{I}_{\mathrm{o}}=$ incident photon flux

$\mathrm{I}=$ transmitted photon flux.

In terms of the photocurrents of the array diodes and the associated preamplifier circuitry, the above equation is:

$$
A(\lambda)=\log \frac{I_{o}}{I}=\log \frac{I_{\text {ref }}+I_{1}{ }^{\prime}+I_{2}{ }^{\prime}+I_{3}{ }^{\prime}}{I_{\text {sample }}+I_{1}+I_{2}+I_{3}}
$$

where $\mathrm{I}_{\text {ref }} \quad=$ photocurrent reference signal

$\mathrm{I}_{\text {sample }}=$ photocurrent sample signal

$\mathrm{I}_{1}{ }^{\prime}, \mathrm{I}_{1}=$ photocurrents produced by stray light

$\mathrm{I}_{2}{ }^{\prime}, \mathrm{I}_{2}=$ additional offset currents produced by the signal electronics

$\mathrm{I}_{3}{ }^{\prime}, \mathrm{I}_{3}=$ dark currents caused by the photodiodes.

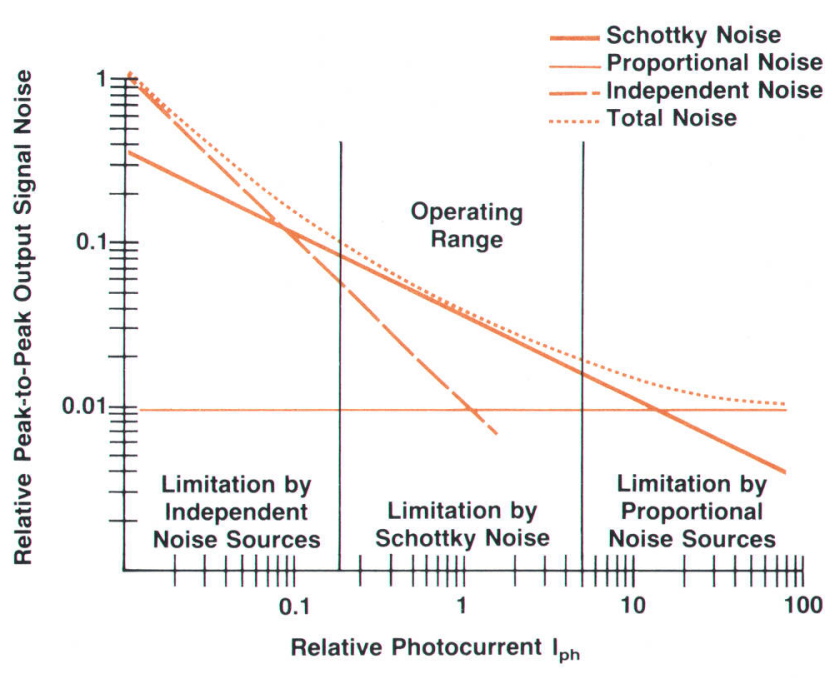

Fig. 1. Output signal noise of a diode array detector as a function of photocurrent. 
The absorbance signal can be corrected for offset and dark currents simply by measuring these currents during an analýsis. Stray light is the main factor limiting the detector's linearity. Stray light is an intrinsic property of wavelength dispersive elements, which means that gratings of the highest quality are absolutely necessary. In the HP 79854A MWD, stray light is less than 1/1000 of the sample light, resulting in a linearity error of less than $1 \%$ up to 1.5 AU.

\section{Flow Cell Trade-Offs}

According to the Lambert-Beer law, a longer path length gives a correspondingly higher signal. However, longer path lengths result in larger volumes, increasing the dispersion of the separated peaks. Reducing the volume with a long path length reduces the light throughput, corresponding to a relatively higher (absorbance) signal level, but at a higher noise level. A further constraint comes from an "Iron Rule" of chromatography, which says that no more than $10 \%$ of the original resolution must be lost in the detection system. This means that the volume of the flow cell must be:

$$
\mathrm{V}_{\text {cell }}<0.5 \frac{\mathrm{V}_{\mathrm{r}}}{\mathrm{N}}
$$

where $\mathrm{V}_{\mathrm{r}}=$ retention volume

$\mathrm{N}$ = number of theoretical plates (a column parameter).

For these reasons, all absorbance detector designs are trade-offs between path length, cell volume, and total photon flux, which is controlled by the element with the smallest light conductivity, the flow cell.

\section{Signal-to-Noise Ratio}

In addition to the path length of the flow cell and the dispersion of the eluted peak, signal-to-noise ratio is a limiting factor in detector performance.

In modeling the noise contributions in a photometric absorbance detector, three types of noise need to be considered: Schottky noise, independent noise, and proportional noise. There is also a relationship between photometric noise (noise associated with the photometric signal) and output signal noise:

$$
\underset{\text { Noise }(\mathrm{AU})}{\text { Output Signal }}=\frac{\text { Photometric Noise }}{2.3 \times(\text { Photometric Signal })}
$$

Schottky Noise. Schottky noise is always associated with direct current flow, for example in diodes and transistors. In this case the Schottky noise is associated with the photocurrent generated by the photodiodes. The rms Schottky noise current $\mathrm{I}_{\mathrm{s} \text { rms }}$ on a photometric signal $\mathrm{I}_{\mathrm{ph}}$ is:

$$
\mathrm{I}_{\mathrm{s} \text { rms }}(\text { amperes })=\sqrt{2 \mathrm{qI}_{\mathrm{ph}} \mathrm{BW}} \text {, }
$$

where $\mathrm{q}$ is the charge of the electron and BW is the electrical bandwidth. In detectors, it is more convenient to work with the peak-to-peak value of the noise, which is approximately

$$
I_{\text {s p-p }}(\text { amperes })=6 I_{s ~ r m s}=6 \sqrt{2 \mathrm{qI}_{\mathrm{ph}} \mathrm{BW}} .
$$

The response on the chromatographic baseline is:

$$
I_{\text {s p-p }}(A U)=\frac{I_{s p-p} \text { (amperes) }}{2.3 \mathrm{I}_{\mathrm{ph}} \text { (amperes) }}=\frac{6 \sqrt{2 \mathrm{qI}_{\mathrm{ph}} \mathrm{BW}}}{2.3 \mathrm{I}_{\mathrm{ph}}} .
$$

Independent Noise. This noise term is seen as a fixed amount of noise superimposed on the photometric signal. It is mainly generated by the electronics (amplifiers, analogto-digital converters, etc.) and is independent of the level of the photometric signal. It can be expressed as:

$$
\mathrm{I}_{\mathrm{i} \mathrm{p} \text {-p }}(\text { amperes })=\text { constant } \neq \mathrm{f}\left(\mathrm{I}_{\mathrm{ph}}\right) \text {. }
$$

The response on the chromatographic baseline is:

$$
I_{i ~ p-p}(A U)=\frac{I_{i p-p}(\text { amperes })}{2.3 I_{p h}(\text { amperes })}
$$

Proportional Noise. This noise term is proportional to the photometric signal. It comes mainly from lamp instabilities and gain variations in the electronics. It can be expressed as:

$$
\mathrm{I}_{\mathrm{p} \mathrm{p-p}}(\text { amperes })=\mathrm{kI}_{\mathrm{ph}}=2.3 \times 10^{-5} \mathrm{I}_{\mathrm{ph}}
$$

The response on the chromatographic baseline is:

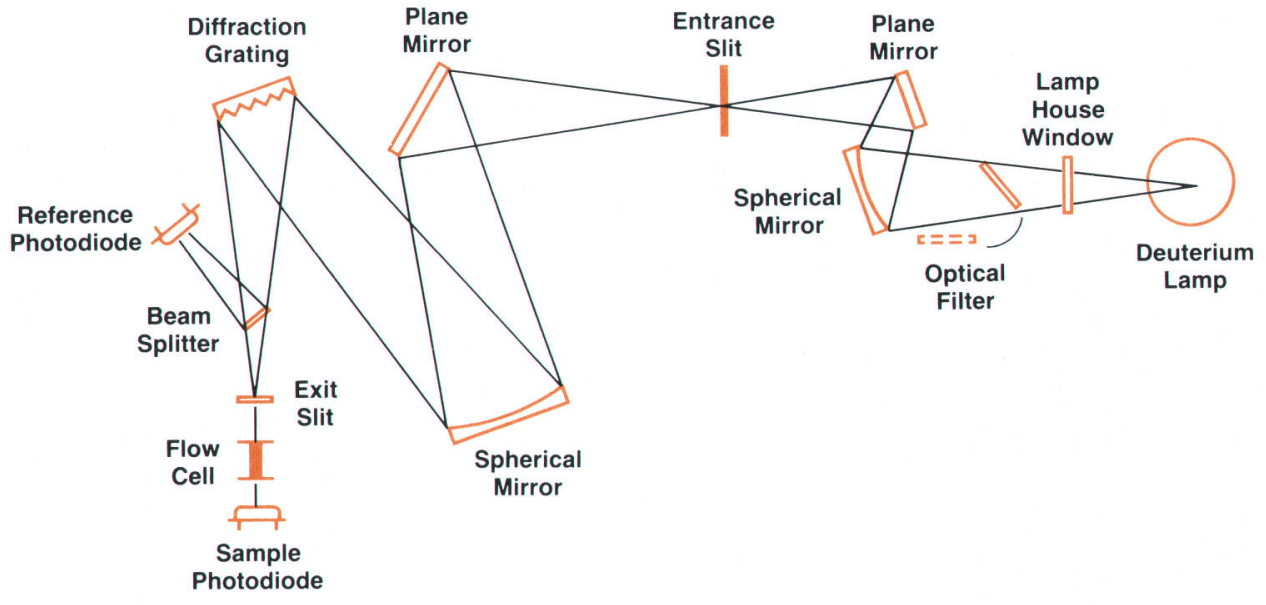

Fig. 2. Optical system of the HP 79853A Programmable Variable Wavelength Detector, a forward optics, scanning absorbance detector. 


$$
I_{p \text { p-p }}(A U)=\frac{I_{p p-p}(\text { amperes })}{2.3 I_{p h}(\text { amperes })}=\frac{\mathrm{kI}_{\mathrm{ph}}}{2.3 \mathrm{I}_{\mathrm{ph}}}=\frac{\mathrm{k}}{2.3}=10^{-5}
$$

Total Noise. The total noise on a chromatographic signal is:

$$
I_{t}(A U)=\sqrt{I_{s}^{2}+I_{i}^{2}+I_{p}^{2}} \text {. }
$$

The result of this model is shown in Fig. 1. Within the operating range of the detector, the total noise of the system approaches very closely the theoretical limit, which is the Schottky noise.

\section{Variable Wavelength Detector}

Features of the HP 79853A Variable Wavelength Detector include high sensitivity with low noise and drift, and versatility in the form of programmable wavelength, autozero capability, and interchangeable cells. This detector has a single-channel output. Time-programmable wavelength switching and stop-flow scanning make it possible to take a spectrum to find a wavelength with optimum sensitivity for a given chromatographic peak, thereby achieving maximum sensitivity.

\section{Scanning Absorbance Detector Optics}

In the optical system of this detector (Fig. 2), radiation from a deuterium lamp is focused on the entrance slit of a monochromator by means of spherical and plane mirrors. In the monochromator, a 1200-line/mm grating in a highly efficient Monk-Gillieson optical system spatially disperses the beam and focuses the selected portion of it on the exit slit, which has an 8-nm optical bandwidth. The beam passes through a flow cell which has an $8-\mu$ l volume and a $10-\mathrm{mm}$ path length, and the transmitted light is converted by a sample photodiode into an electrical signal. To compensate for intensity fluctuations of the light source, a beamsplitter in the monochromator directs part of the light to a reference photodiode. Wavelength selection is made by rotating the grating, which is driven by a stepper motor. An optical unit casting contains all items: mirrors, beamsplitter, grating assembly, and two photodiode assemblies. The deuterium lamp is located in a separate compartment attached to the casting.

Flow cell construction follows a classical steel cell concept. A steel housing contains the Suprasil windows, gaskets, screws, and other parts. The inlet capillary is coiled around the housing, forming an efficient heat exchanger. The assembly is part of a cassette that can be removed from the instrument for cell inspection. A simple sheet-metal case, which follows HP standard appearance design guidelines, contains all subassemblies: the optical unit with its cell cassette, the power supply, analog-to-digital and digital-to-analog converters, the microprocessor, and the user interface.

\section{Data Acquisition and Processing}

One of the main goals in designing a signal path is to find a solution that provides operation as near as possible to the theoretical noise limit, which is the Schottky noise of the photocurrent. This means that the electronic circuits, including the light source and the photodiodes, must add a minimal amount of noise above this limit. Other goals are to provide signal filtering matched to the chemical ap-

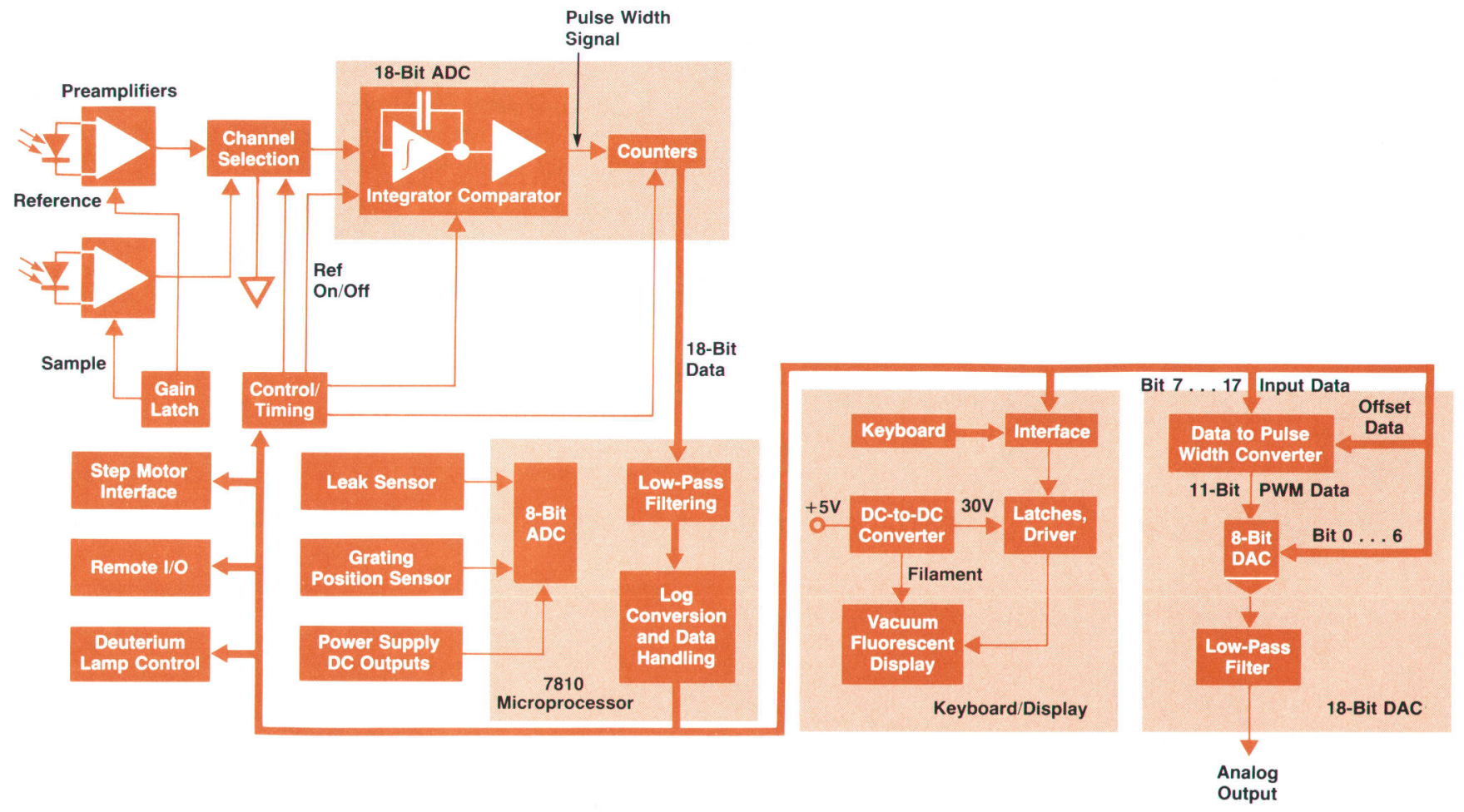

Fig. 3. Signal processing system of the HP 79853A Detector. 
plications and time tables to change parameters during an analysis. Fig. 3 shows the block diagram of the HP 79853A Detector. Light energy is converted to photocurrent by silicon pin photodiodes, which generate photocurrents as large as $10 \mathrm{nA}$. Because the light intensity varies significantly with wavelength, the preamplifier gain is increased from a normal value of 1 to 2,4 , or 8 depending on the actual photocurrent. The next stage is a channel selector connecting the analog-to-digital converter (ADC) input to the sample or reference photodiode or to analog ground for offset compensation measurement. The ADC is an integrating type with a basic conversion frequency of $1.2 \mathrm{kHz}$, generating a pulse width signal proportional to the input voltage. Twenty-four conversion cycles and some waiting time are summed, resulting in an 18-bit ADC with 25-ms conversion time. This speed is more than adequate for LC purposes, even for the fastest chromatographic applications.

As mentioned earlier, compensation using a reference photodiode provides advantages in suppressing commonmode effects. It is best to use identical parts for measuring the sample and reference photocurrents. Therefore, only the photodiodes and the first op amps are individual parts. All other parts in the signal path, including the ADC, are identical. With this design it is possible to guarantee drift values of less than $5 \times 10^{-4}$ AU per hour.

A 7810 one-chip microprocessor clocked at $12 \mathrm{MHz}$ is used for all control and data handling operations. Corresponding to the user-selected signal response time $(0.25,1$, or 4 seconds) the 18-bit ADC data is low-pass filtered and converted to absorbance data according to the equation

$$
\mathrm{A}(\lambda)=\log (\text { reference data })-\log (\text { sample data })
$$

This does not normally result in $\mathrm{A}(\lambda)=0$ for a nonabsorbing probe, as required by the Lambert-Beer law, because the solvent itself and the cell absorb some light. To compensate, a balance is performed. Just before starting an analysis the actual absorbance is measured and stored, and all future absorbance computations are corrected by this value.

The data output of the CPU consists of two different types: data to be displayed and data for the analog output signal of the detector. The display is a 16-character singleline vacuum fluorescent display with $5 \times 7$ dot matrix characters. For the analog output signal, a digital-to-analog converter (DAC) operates on the digital data.

The dynamic requirements of the DAC are determined by signal and noise considerations. The value represented by one least-significant bit must be small compared to the minimum signal level, which here is noise. The full-scale analog output corresponds to $2 \mathrm{AU}$ (by definition), and the minimum noise is $2 \times 10^{-5} \mathrm{AU}$. The result is that $1 \mathrm{LSB}$ must be less than $1 \times 10^{-5} \mathrm{AU}$ or the dynamic range must be greater than 200,000. Consequently, an 18-bit DAC is needed. To obtain this dynamic range with monotonic behavior, a two-stage DAC principle was chosen. The first stage is an 11-bit pulse width modulated DAC with 20-bit accuracy operating at a $183-\mathrm{Hz}$ repetition rate. The output of this stage enables 7 bits of a second monolithic DAC. The two DAC outputs are added, resulting in the required 18 bits. Low-pass filtering suppresses the ac content to a negligible amount

Besides signal data handling, the microprocessor has some further tasks to perform. For safety reasons, a leak detector near the cell is monitored with a built-in 8-bit ADC. In case of any malfunction, appropriate not-ready and error messages are activated. The microprocessor also generates the bit pattern for the step motor to change the wavelength, and takes care of deuterium lamp control, shutter movement for suppression of optical second-order diffraction (optical low-pass filter), and remote control input and output for synchronizing the operation of all connected LC modules.

\section{Detector Control}

Users of LC detectors are commonly chemists or persons in chemical labs. They need easy access to the most important functions with a self-explanatory display and keyboard. This is quite difficult because the space for the keyboard is limited and because access to 26 different parameters and functions must be provided. In addition, about ten special functions for service purposes are available. In the HP 79853A, the most frequently needed functions are directly accessed via corresponding keys (deuterium lamp on/off, start/stop analysis, wavelength selection, and balance). The remaining functions are accessible via a special control key plus an identification number. All control functions can be reached either in this way or by scrolling forward and backward with up and down keys. The first five control functions are those related to spectrum acquisition and spectral data output. There are also automated functions allowing tasks like finding the wavelength with maximum absorbance in a measured spectrum and switching on or off the deuterium lamp after a predefined time interval to extend the life of the lamp.

\section{Multiple Wavelength Detector}

The HP 79854A Multiple Wavelength Detector is designed for customers who need not only signal sensitivity but also spectral sensitivity and more information. This detector features simultaneous dual-wavelength monitoring and on-line peak purity checks.

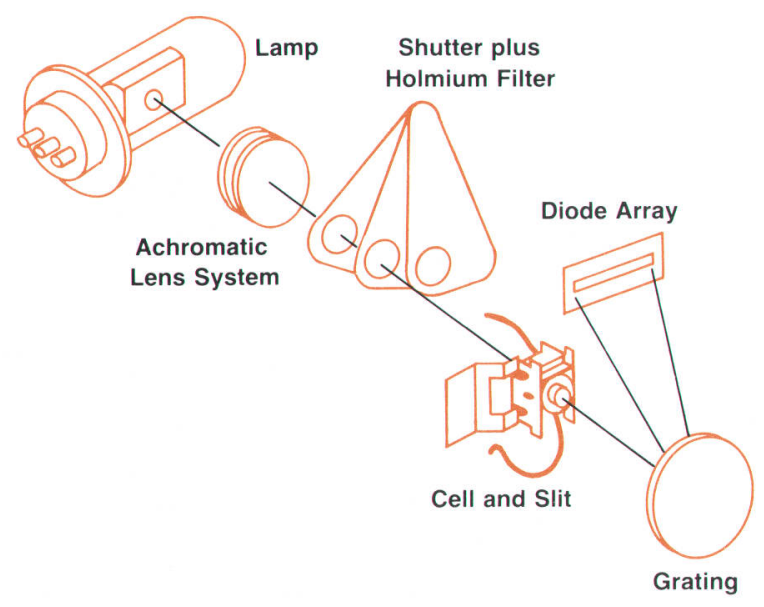

Fig. 4. Optical system of the HP 79854A Multiple Wavelength Detector, a reverse optics, diode array absorbance detector. 
The main objectives for the development team were to achieve the highest possible sensitivity in terms of noise, wander and drift, to reduce spectral noise to its theoretical minimum, to make the instrument insensitive to refractive index changes with temperature, and to implement enough functionality to make the instrument a useful tool in both research and quality assurance labs.

\section{MWD Optical System}

Fig. 4 shows the optical system of the HP 79854A. The radiation source is a deuterium discharge lamp that has a wavelength range of 190 to $600 \mathrm{~nm}$. Light is collected by an achromatic lens system and directed onto the spectrometer slit, which is $120 \mu \mathrm{m}$ wide and $635 \mu \mathrm{m}$ high. In the plane of the slit a 1:2 reduced image of the lamp aperture is formed. The slit is part of the cylindrical flow cell, which is made of quartz and has a path length of $6 \mathrm{~mm}$ and a cylindrical volume of $8 \mu \mathrm{l}$. By designing for an optimum fit between solid angle, image size, and flow cell geometry, wall effects (reflections at the cell walls and refractive index sensitivity) are avoided and a high photon flux is achieved. ${ }^{1}$

A holographic concave grating disperses the emergent radiation linearly onto a photodiode array. The array consists of a row of 211 individual silicon photodiodes with a center-to-center spacing of $61 \mu \mathrm{m} .205$ diodes are used for the $410-\mathrm{nm}$-wide wavelength range, so the dispersion factor is $2 \mathrm{~nm}$ per diode. Because of the $120-\mu \mathrm{m}$ slit width the signal bandwidth is $4 \mathrm{~nm}$, a trade-off between spectral resolution and high light throughput. ${ }^{1} \mathrm{~A}$ cut-off filter placed before the array removes second-order spectra.

The required spectral dispersion as a function of wavelength determines the groove density of the grating. Grating aberrations - spectral plane focus, astigmatic focus, and coma-have to be minimized over the spectral range of interest. Fabrication of a flat-field master grating is done via holographic recording techniques, that is, a 3D interference pattern is produced by two coherent point sources (their positions can be calculated from known imaging properties and the required dispersion). By means of an appropriate groove profile the amount of light into a desired spectral order can be controlled (70\% efficiency at $225 \mathrm{~nm}$ ). Final grating production is by replication processes.

A shutter positioned between the lens system and the flow cell can cut off the radiation for dark current compensation or interpose a holmium oxide filter into the radiation path. With its characteristic spectrum, the filter calibrates the photodiode array for a correct wavelength scale.

The casting containing all optical components is mounted on a sheet-metal base. The cell area and all capillaries are easily accessible by opening the front door. All electronics, including the power supply, ADC and DAC, data acquisition unit, and central processing unit, is located behind the optics in the rear half of the box and is easily accessible for service and upgrades.

For a good noise profile, the temperature variation in the optical setup needs to be as low as possible. Heat dissipation occurs at the lamp position $\left(100^{\circ} \mathrm{C}\right)$ and in the cell, the latter caused by the flow of solvent previously heated in the LC column oven (up to $100^{\circ} \mathrm{C}$ ). There is also dissipation over the capillary steel walls. Active cooling is done separately by two fans. The first is adjacent to the lamp compartment, and the second is behind a large heat exchanger. Both take in air from the front of the box. A small heat exchanger is attached directly to the cell for fine-tuning the temperature profile.

\section{Signal Electronics}

The self-scanning photodiode arrays are built on silicon semiconductor material and consist of a number of photocells connected to a common output (video line) by means of electronic switches. The switches of the individual photocells are controlled by a shift register so that the photocells are read sequentially according to the shift register clock signal. The common output is connected to a charge amplifier.

A schematic diagram of a self-scanning photodiode array is shown in Fig. 5. Each photocell has an associated capacitor $\mathrm{C}_{\mathrm{di}}$, which represents the junction capacitance

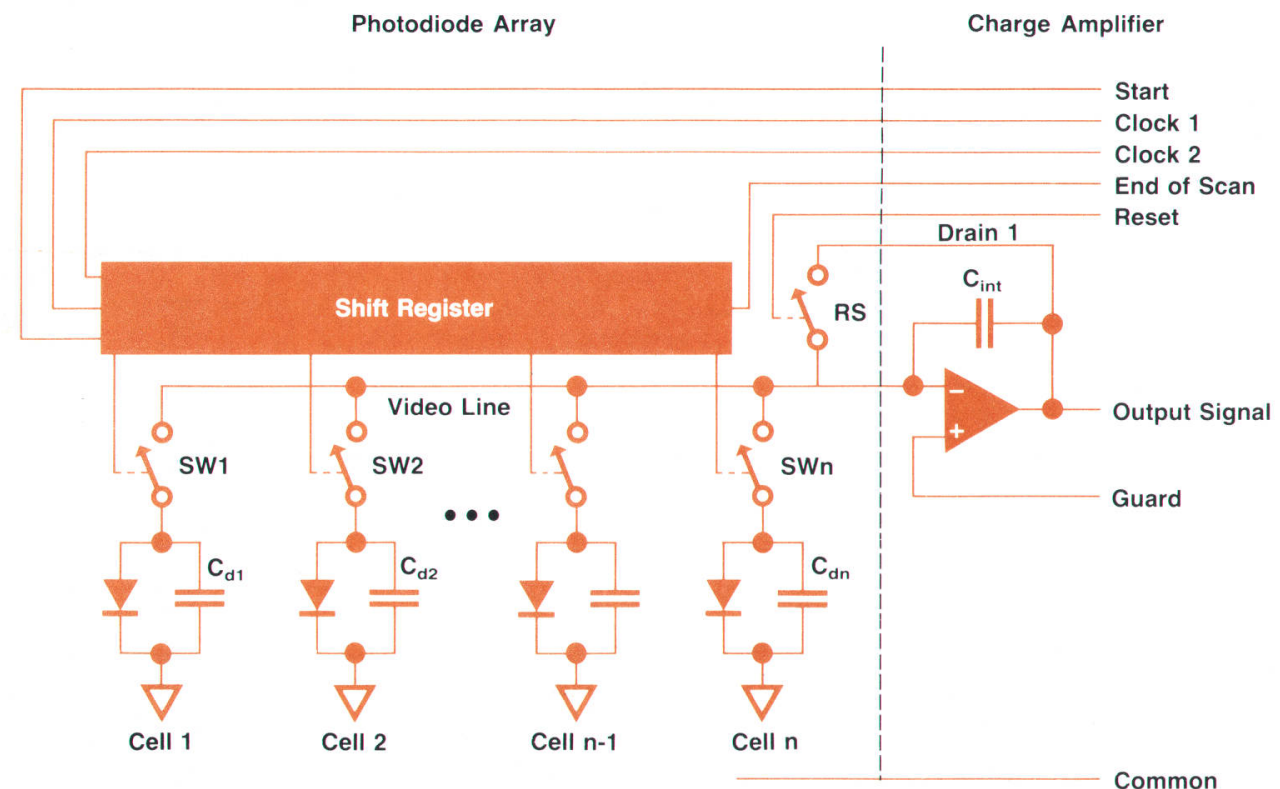

Fig. 5. Schematic diagram of a self-scanning photodiode array. 
of the photodiode or, in some cases, is a capacitor separately added on the chip. In operation, the capacitors of the photocells are initially charged to a fixed value by scanning all photocells.

When photons penetrate the photosensitive material, charge carriers are generated, which discharge the capacitors according to the number of photons within a given integration period. When a particular photocell is connected to the common output, its capacitor is recharged from the charge amplifier and the amount of charge required represents the discharge level of that cell's capacitor, which is proportional to the incident light level during that integration period. Before each charge transfer from the charge amplifier to a photocell, the reset switch RS is closed to null the charge amplifier and prepare it for the next transfer.

The charge amplifier integrates its input signal so that the voltage change at its output is proportional to the integral of the incident light level during the integration period. This signal is further processed by an amplifier, a sampleand-hold circuit, an analog-to-digital converter (ADC), and a microprocessor.

\section{Analog-to-Digital Converter}

The ADC combines the benefits of the successive approximation technique and the advantages of the dual-slope algorithm. The benefits of successive approximation are high resolution of 16 bits, or steps of $76 \mu \mathrm{V}$ over a $5 \mathrm{~V}$ input range, and a high conversion rate of 18,000 readings per second. The dual-slope algorithm provides low noise, a good temperature coefficient, excellent differential linearity, and low manufacturing costs. The ADC is only required to make relative, not absolute, voltage measurements. The dual-slope-based conversion principle is called tripleslope integration because of the three different parts of the integrator voltage waveform (Fig. 6).

A schematic drawing of the ADC is shown in Fig. 7. The input voltage coming from the photodiode array passes through a level shifter and a variable-gain stage before charging the integration capacitor. A very fast comparator detects the zero crossings of the integrator voltage (critical areas around the zero level are amplified to avoid comparator toggling) and triggers the digital timing logic, which switches the discharge currents and controls the counters. After the conversion the corrected counter content is

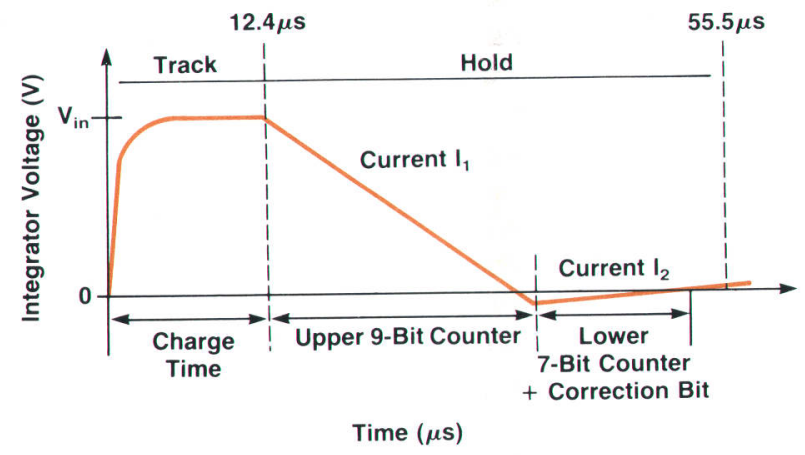

Fig. 6. Integrator voltage waveform for triple-slope integration. loaded into the output data register as the 16-bit conversion result.

One difference between the triple-slope integration technique and common dual-slope techniques is that the integrating capacitor is charged by a constant voltage instead of by a voltage dependent current during a fixed charge time. Therefore, no autozero step is necessary between readings to remove the charge remaining from the previous conversion. Thus the number of error-inducing high-speed switches is minimized and a typical overall noise level of \pm 0.5 LSB (least-significant bit $=76 \mu \mathrm{V}$ ) is achieved.

The other main difference solves the great disadvantage of the dual-slope principle, the relatively slow conversion rate, by using two bipolar discharging currents with a constant ratio of $I_{1}=-128 I_{2}$. The conversion rate is increased by a factor of about 64 . An internal correction bit makes it possible to eliminate various switching delay errors by providing additional time to drive the integrator voltage to the zero level.

The capacitor discharge times are measured by a single comparator circuit to eliminate offset voltages. The output digital data word is the result of multiplying the upper nine data bits by the weight of 128 and adding the correction bit and the lower seven data bits.

To achieve the full speed advantages of this technique, a pipelined signal flow prepares the next diode voltage to be switched to the integrator while the current conversion is running. At the same time the data word resulting from the previous cycle is kept in the output latches to be fetched by the front-end microprocessor. This pipelining concept

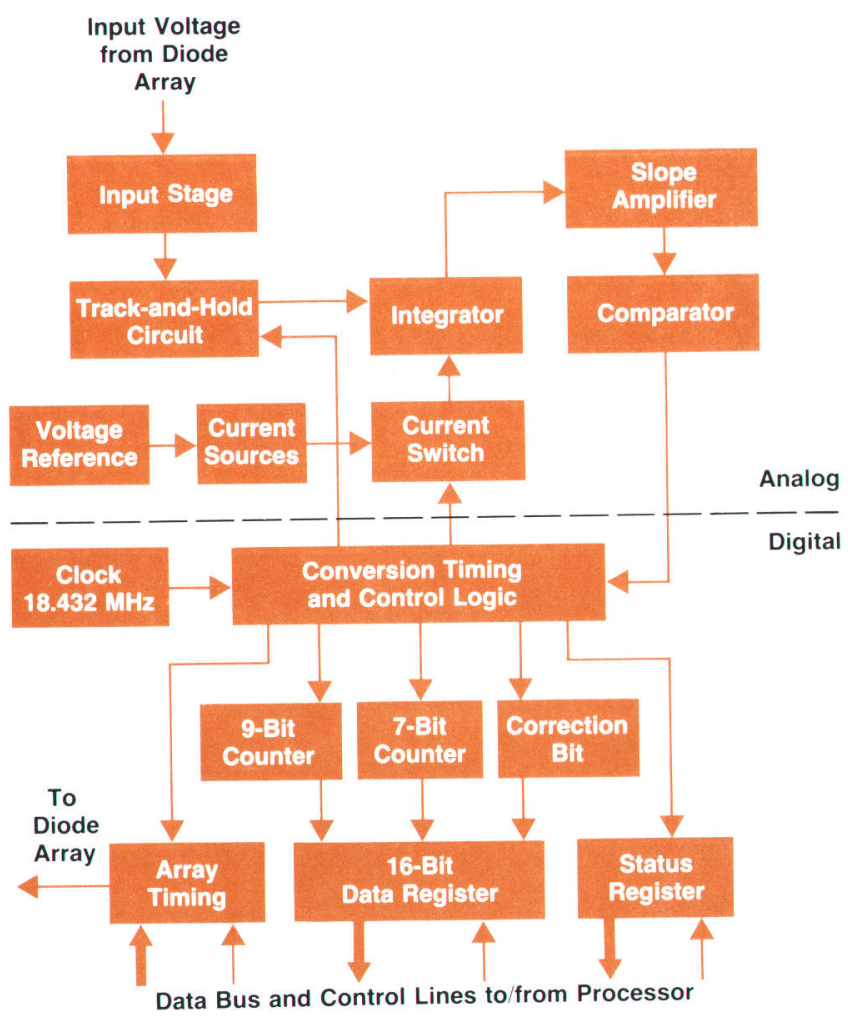

Fig. 7. Block diagram of the analog-to-digital converter (ADC) of the HP 79854A detector. 
reduces the timing requirements for the processor enormously by keeping the computed result stable for the time needed for a full conversion cycle.

A small on-board diagnostic circuit is provided to test the ADC with two constant internal voltages and an additional ramp voltage covering nearly the full input range, using only the digital output data as test results. Thus a good ADC test is possible even if there are malfunctions elsewhere in the electronics.

\section{Data Processing and Architecture}

Processing is split between a small preprocessor system and the main processor system (see Fig. 8). The preprocessor takes care of array data readout and control of the diode array. The main processor is responsible for data calculation, I/O, and user interface handling.

HP's first fast scanning detector, the multiwavelength HP 79880A, had a similar architecture, using a fast bit-slice front-end processor and a Z80A main processor. For the new HP 79854A, the costly and chip-consuming bit-slice preprocessor is replaced by a standard 6809 preprocessor. The main processor is a standard 68008. Performance is maintained, but the chip count is reduced by two thirds, reliability is increased, and the cost is reduced by one third.

The 6809 preprocessor system controls the array and gain switching hardware to set the correct gain for each diode, and it synchronizes the array cycle with the 12.5-ms data processing cycle. It also captures data coming from the ADC with a period of $55 \mu \mathrm{s}(18,000$ words/s), filters the values separately for each diode, and block-transfers 20 raw intensity-scan records per second to the main processor.

The 68008 main processor, running under a multitasking operating system, handles the local keyboard input, display output, and DAC control and does the complex calculations necessary to provide chromatographic signals and spectra at the analog outputs. Since the main-processor load is critical, the main processor is interrupted by the preprocessor only when a complete scan record is available for processing.

The main processor corrects the raw intensity data from the preprocessor for dark and electronic offsets. The corrected record represents the light intensity distribution over the wavelength range from 190 to $600 \mathrm{~nm}$. This record, called a raw intensity scan, is used to build chromatographic signals and spectra. Six raw signals are generated by bunching (adding) intensities in the wavelength domain to get the desired center wavelength and bandwidth. These raw signals are filtered in the time domain according to the required peak width, that is, the filter length and output data rate are set to produce the number of data samples needed to reconstruct the peak shape, height, and area correctly. To get the absorbance values, the filtered raw signals are log-converted and referenced to the background. Finally, the data rate is converted from the current sample rate to the DAC output rate to make the chromatographic absorbance signal available for analog output.

As shown in Fig. 8, the first step in spectrum processing is bunching the intensity data for each wavelength in the time domain up to the desired acquisition time, which also depends on the peak width. Next, the logarithm is calcu- lated and the background scan is subtracted. The result, the absorbance spectrum, is stored in spectrum memory, which holds up to ten spectra.

Calibration values, used to set the correct gain for each diode and to correct the raw intensity values, are measured during a special calibration cycle on request and the results are stored in control and correction tables for continuous use during normal measurements.

\section{Detector Functions}

The user can predefine three different sample wavelengths and three different reference wavelengths and change them in a time program. Unwanted impurities within a chromatogram can be subtracted from the sample signal by simply setting the reference to a wavelength where only an impurity spectral band exists. Arithmetic is possible not only with signals but also with spectra. Spectra are taken instantaneously on demand or can be programmed and stored. Subtraction of spectra from the same or other runs (baseline spectra) is done off-line.

For identification of unknown compounds, a quick means of creating a chromatogram with the highest signalto-noise ratio per peak is desirable. Diode array technology

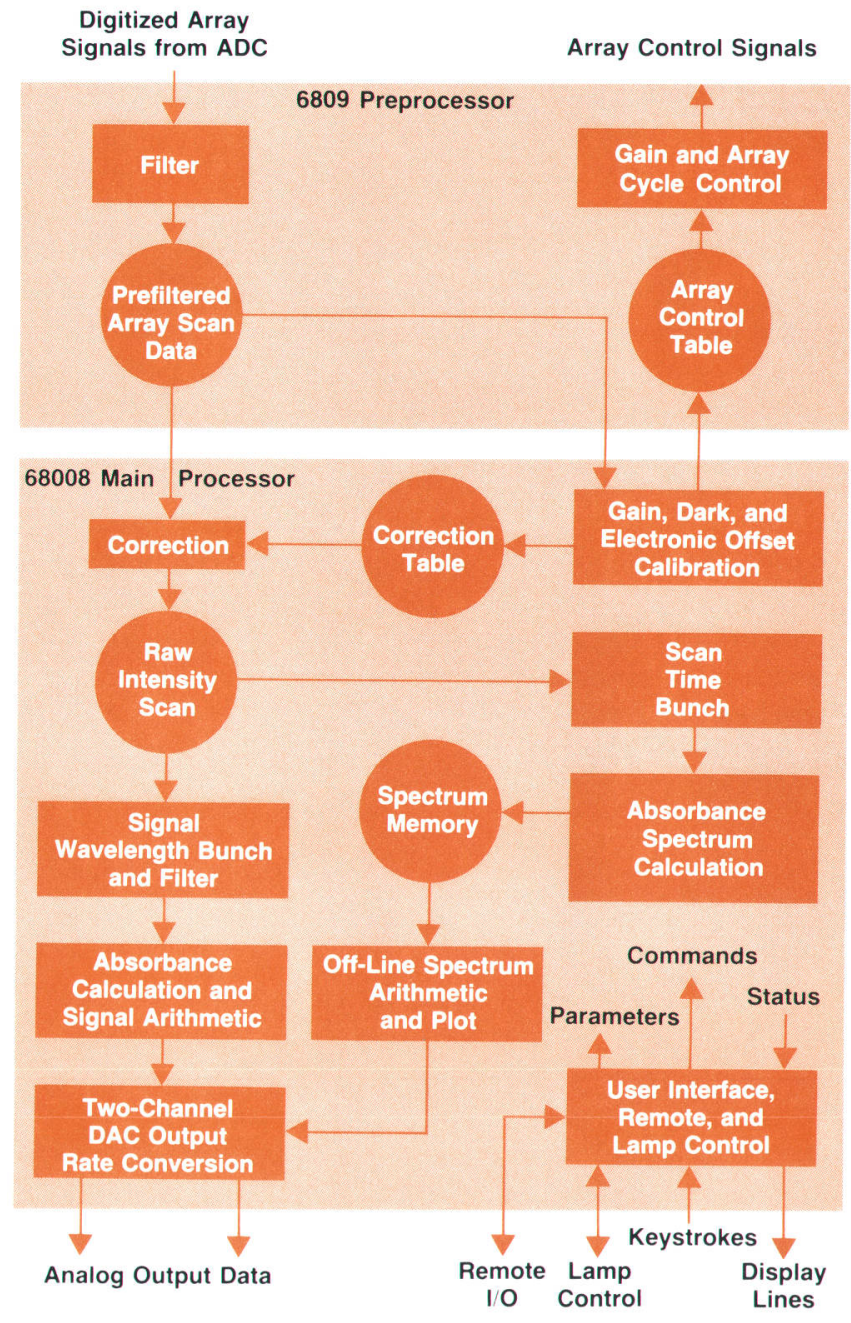

Fig. 8. Signal processing in the HP 79854A Detector 
offers on-line information about the wavelength with the highest absorbance in the cell for each peak. This helps in choosing the right wavelengths for a successful time program.

\section{Troubleshooting}

Unfortunately, the detector can't tell whether unnatural noise or signal behavior is caused by an LC system malfunction or a detector failure. For example, the noise might be too low or too high, the baseline might be randomly or regularly spiked, or microwander and jumps might occur. For the multiwavelength detector, troubleshooting starts by exchanging the flow cell with the test cell and cutting off the flow path from the detector. Then the detector selftest or various test functions can be invoked. Error messages, such as leak detected, error in other module, lamp ignition failed, low intensity, or wavelength calibration out of range, may be helpful in correcting a malfunction. There is a lamp intensity test to check the output of the light source. The holmium spectrum can be measured to monitor stray light in the UV range, and for an electronic noise test the lamp is disconnected and the signal path from the diode array to the electronics is checked. The printed circuit boards have their own specialized tests. The gain factors of the ADC can be measured, and the DAC can be tested using a built-in test pattern as input.

\section{Acknowledgments}

We would like to thank Bernhard Dehmer who designed the packaging system, Hubert Wilbs who contributed to the optical unit, Claus Lueth who designed the central processing board, and Karsten Kraiczek who helped with the heat exchanger design and test software development. Special thanks to Setsuo Muramoto and his team for the development of the scanning absorbance detector.

\section{Reference}

1. J. Leyrer, et al, "A High-Speed Spectrophotometric LC Detector,” Hewlett-Packard Journal, Vol. 35, no. 4, April 1984. 


\title{
Firmware Development for a Modular Liquid Chromatography System
}

\author{
More than half of the firmware for the HP 1050 Series High- \\ Performance Liquid Chromatography System is common \\ to all modules. It is customized for individual modules by \\ means of module-specific tables.
}

\section{by Christian Büttner, Fromut Fritze, and Gerhard Ple}

ACH HP 1050 SERIES MODULE is a stand-alone unit, performing one specific task required in a liquid chromatography system. There are three types of modules: solvent delivery system, automatic liquid sampler, and detector. Combined, the modules form a complete, working analysis system. Therefore, the scope of the firmware implementation is the combined functionality of a complete LC system, but the firmware physically resides in specific modules.

Based on our experience with earlier products and the large number of new functions, which had to be implemented by up to ten engineers working in parallel within a time frame of two years, we took the trouble to establish a robust firmware development process with some significant new approaches. Some key objectives were to reuse as much code as possible, to aim for easily maintainable code, and to insist on identical processor and user interface hardware for all modules.

Code Reuse. One of the most important goals was code reuse. We adapted design concepts from a previous product and emphasized strongly common solutions for all devices. For common functions, we aimed to reuse code unmodified from device to device. Even functions that only have common concepts got identical code and are tailored to the device-specific needs by tables.

Easy Maintainability. Both to remove defects and to enhance the product's functions, postrelease work may be necessary on the code. In addition, the control firmware for future instruments may be derived from an existing version. This motivation affected the structuring of the firmware and led to the decision to write code in Pascal, limiting the amount of assembly language code to the absolute minimum.

Identical Hardware. A prerequisite to making and handling a large amount of common code is the use of identical keyboards, displays, and microprocessor architecture in all modules. Fig. 1 shows the generic electronic hardware block diagram. The processor board, which is common to

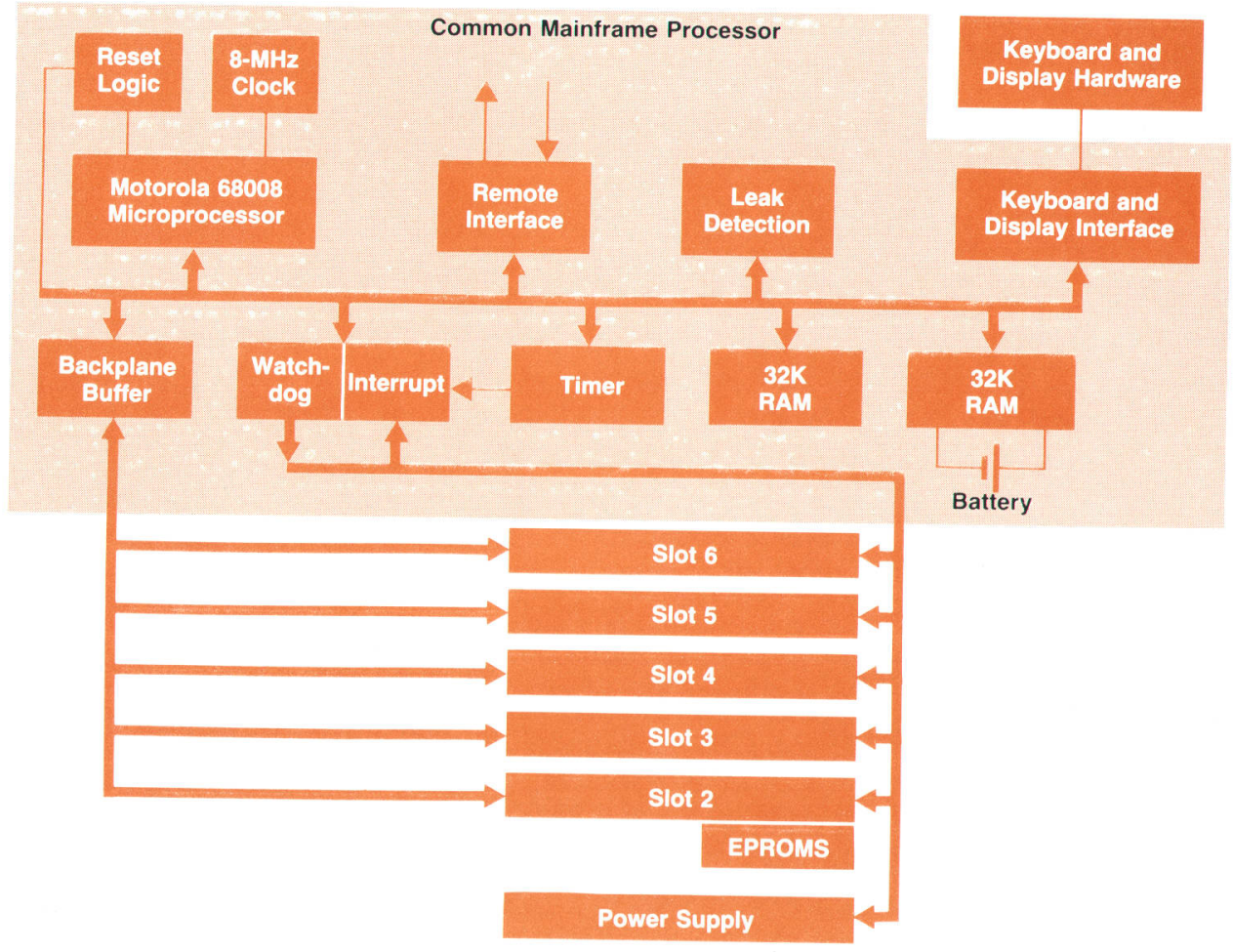

Fig. 1. Block diagram of the generic HP 1050 Series module electronics 
all modules, occupies one slot of the cardcage. It is based on a Motorola 68008 microprocessor, has $64 \mathrm{~K}$ bytes of RAM with $32 \mathrm{~K}$ battery powered, and can handle up to $512 \mathrm{~K}$ bytes of ROM. To keep the processor board absolutely device independent, the ROM is physically located on a device-specific board in another slot. Integrated on the processor board is the remote interface circuitry. The remote interface provides common communication lines between the HP 1050 modules. Additional electronics monitor a safety sensor to detect solvent leaks.

Fixed address ranges are allocated to the different printed circuit board slots (Fig. 2). They range in size from $128 \mathrm{~K}$ or $64 \mathrm{~K}$ for option slots to $16 \mathrm{~K}$ for device-specific slots. The internal bus system is designed to accept additional processor cards in all option slots. The bus system includes all the necessary signals for data, addresses, interrupts, resets, monitoring, and control.

\section{Development Environment}

The firmware can be classified as either hardware independent or tightly coupled to the hardware. For software development we had a number of UNIX workstations and terminals, while we used emulators for integration and tests on the target hardware. In addition, we used a central UNIX machine for file archiving. All UNIX machines were linked by local area networks, while the emulators were linked via a high-speed link. Fig. 3 shows the development system architecture.

Because only a limited number of prototypes and emulators were available, we made every effort to do as much development work on the UNIX workstations as possible. The Pascal compiler for the UNIX workstations and the cross compiler for our target processor had some incompatibilities - for example, different import/export declarations-so to achieve compatibility we built a software pre-

UNIX is a registered trademark of AT\&T in the U.S.A. and other countries

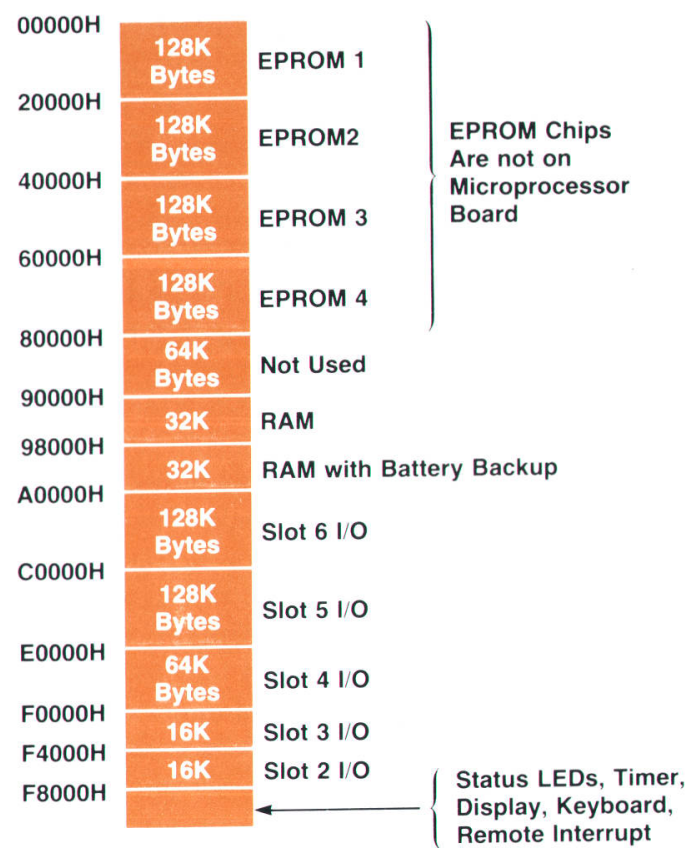

Fig. 2. Common memory map for the HP 1050 Series processor.

processor that handled the differences automatically. This gave us the confidence that a procedure developed on the UNIX workstations could be painlessly transferred to the target hardware without error-prone manual intervention. Since we didn't want to expend too much effort in building a simulation of the target hardware, software verification on the UNIX workstations was restricted to the lesshardware-dependent parts.

Beyond the common UNIX directory structure, each developer had the same user's directory structure to contain

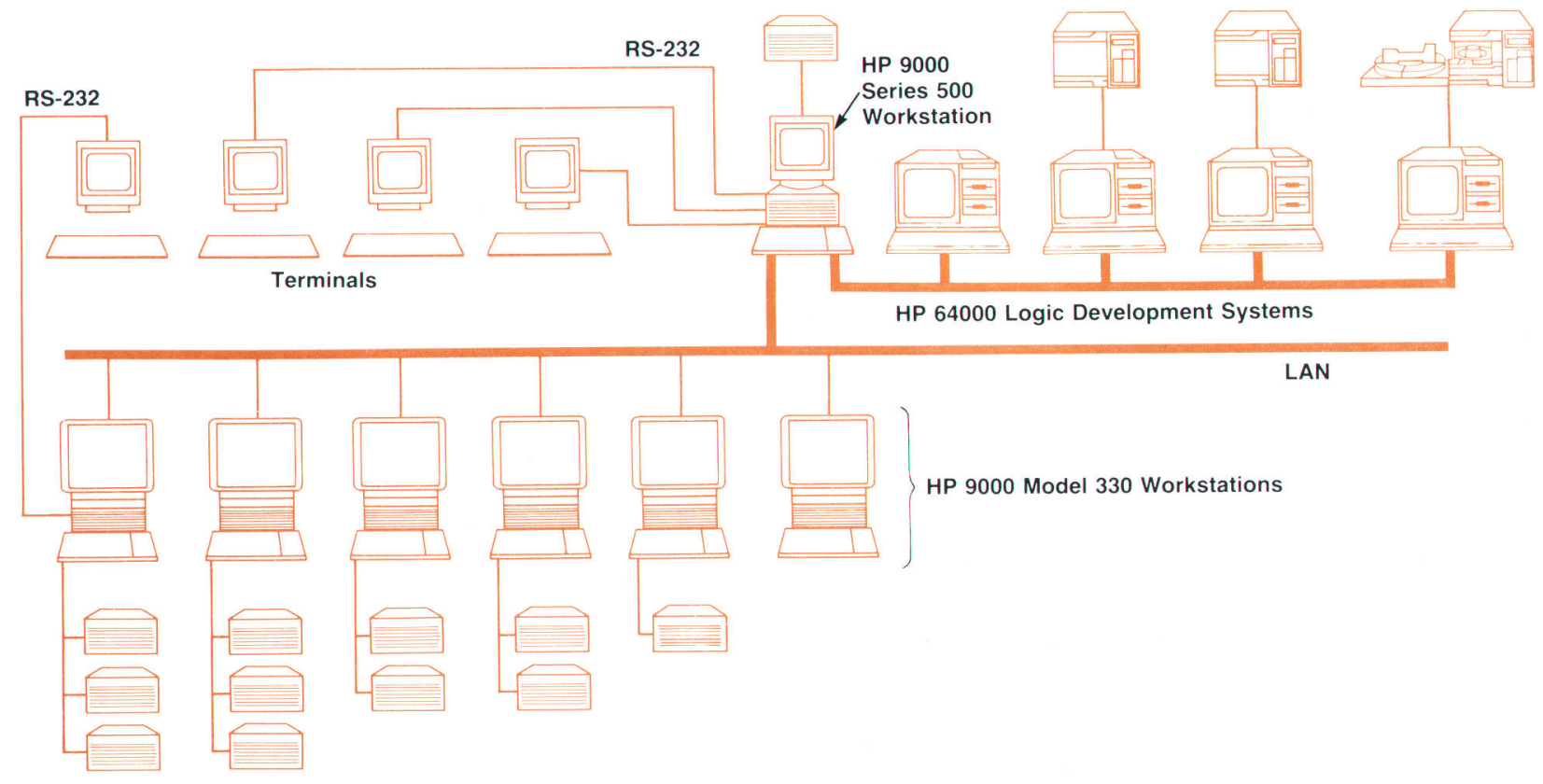

Fig. 3. Workstation and emulator network for development of the HP 1050 Series firmware. 
sources, documents, and tools. The source directories were set up to contain either common code used by all modules or module-specific sources. Fig. 4 shows the directory structure.

To support the Pascal preprocessor we added include and object directories in parallel with each source directory. The include directories contained constants, types, functions, and procedure declarations exported by a given source file. These were automatically generated by the preprocessor and helped to adjust for the different compilers' import/export concepts. The object directories contained either purely UNIX or target-processor relocatable code and made the distinction between the two more obvious.

The preprocessor concept and its import/export requirements had the advantage that we could trace all imports of a given file. Doing this recursively starting with the main program finds all needed sources. Thus, we could easily compile the complete system by just referring to the main program. Alternatively we could automatically check for changed sources and update the affected and dependent files. In the same way we were able to link all necessary object files into an executable file, without the burden of maintaining and updating make files. Again, we just re- ferred to the main program when calling the link utility, and it collected all required relocatables automatically. To add a new source file, we only had to add a new include statement to the calling source file and the tools took care of the compilation and linking.

Because we traditionally use a proprietary multitasking operating system within our firmware, we faced the difficulty of simulating that operating system on top of the UNIX operating system. This allowed us to use the UNIX workstations to verify entire tasks including their communications. Since Pascal doesn't support switching the stack pointer and the program counter (necessary to swap tasks), we used assembly language for our target machines. Unfortunately, no assembler was available for our HP 9000 Series $\mathbf{5 0 0}$ machine, so we had to look for another solution. Thus we used one fork-created process per task on the UNIX workstations. Control between the processes was transferred by UNIX signals while shared memory emulated the target processors' common RAM. Although this isn't as speedy as an assembly language solution for switching contexts, it worked fine for our simulation purposes.

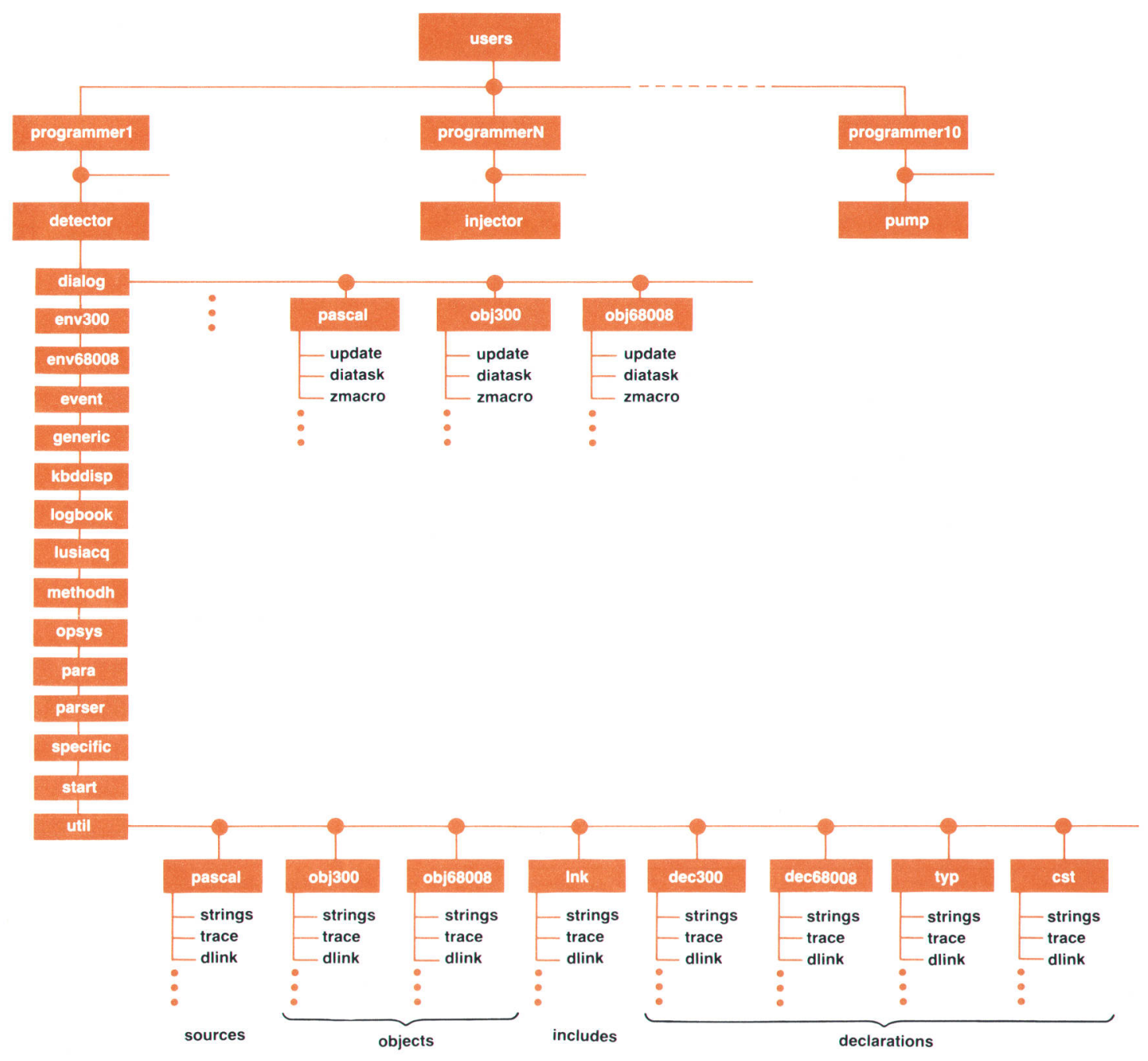

Fig. 4. Directory structure for HP 1050 Series firmware development. 


\section{Revision Control System}

The UNIX revision control system (RCS) was quite heavily used and very valuable, since we had different teams for the various modules working simultaneously. For all functionality we added scripts to access the RCS from any UNIX machine within the network. We also added procedures to check out all files for a given source directory at once. This gave us the ability to maintain software packages as a single source directory (e.g., operating system, dialog, parser, etc.). From time to time, for example when new functionality was added, we had new incompatible versions of a given package. To handle this we simply incremented the major number of that package's internal release and came up with rules like: "For parser version 4.X you need operating system version 5.X." A major benefit of the revision control system is its ability to update a string containing condensed status information for a file version. We put this string into the object code and the final executable code by placing it into a string constant within each file (e.g., c_rcsid = "\$Header: env,v 1.1 89/06/27 10:58:30 ffritze Exp \$”). Thus we had the ability to backtrace the files associated with a given release by simply scanning the executable code or EPROM for that string constant.

\section{Design Overview}

In the beginning of the design process we identified all common function blocks and tried to structure these so that we could easily tailor them to each specific instrument's needs.

Functions were segmented to minimize the interfaces between them. We assigned related functions to tasks handling a given set of data (e.g., parameter handler) or resources (e.g., remote control task). By assigning priorities for the tasks we made the real-time behavior of the module more responsive to hardware and user interaction.

To allow reuse of the common generic tasks and utilities, we had to find a way to adapt their behavior to each instrument's individual needs. To ensure a stable and maintainable platform, we voted against individual modifications of common code. Instead, we designed the tasks so that their behavior could be modified by supplying appropriate information within tables. Good performance is achieved by using mostly hash tables or indexes to address entries rather than interpreting through a whole table. The instruments' behavior was completed by adding specific tasks (e.g., execution tasks) and interrupt routines (e.g., hardware scanner).

\section{Task Structure and Interaction}

The system consists of several independent tasks, as shown in Fig. 5. These tasks and their interactions are managed by a proprietary operating system.

To explain the interaction of the different tasks, let us assume that a user has just pressed a key. This is sensed by an interrupt routine serving as keyboard scanner, which sends a key mail message to the dialog task. Depending on the contents of its tables, the dialog task changes its state, executes some macros, and builds up a new display by sending mail to the display task. The display task thus reflects the entry of the key to the user. In parallel, the update task is informed of a new state and stops its periodic update of the display. Once the user has completed the instruction it is not only echoed by the display, but also sent to the parser. Depending on the contents of its tables, the parser task checks for valid range and builds an internal instruction. This is mailed to the parameter handler, a central task dealing with all instrument parameters. The parameter handler checks the instruction for correctness with respect to other dependencies. To find incompatible settings before execution of a measurement, the instruction is sent to a verify task. Once this check is passed, the setpoint is stored within the parameter handler. An acknowledgment is sent to the parser and forwarded to the dialog task, the display, and the user. If the new setpoint belongs to the currently active parameters (called the active method) it is also transmitted to the execution task. Otherwise, the method handler gathers the settings for later execution.

Simultaneously, depending on the dialog state, the update task may query the parser for actual readings. These requests are forwarded directly to the responsible execution task. The answer is translated backwards by the parser and mailed to the update task, which displays it via the display task. Independently, the hardware is monitored by periodic routines such as the remote control scanner and the leak scanner. Any errors or triggers (e.g., sample injection) are sent to the event handler task, which performs system state transitions (e.g., waiting into run) according to its state table. Triggers may also be logged into the logbook task, while state transitions may be reflected, for

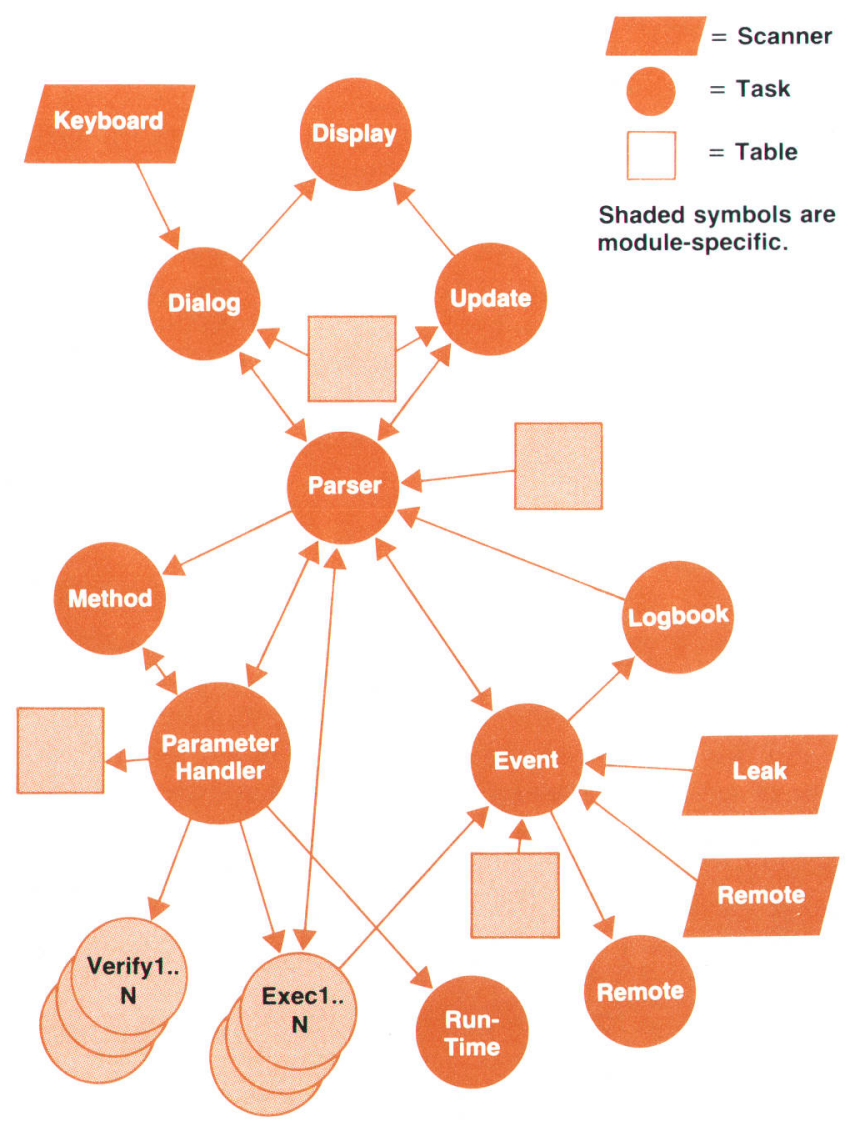

Fig. 5. HP 1050 Series task interactions. 
example, by LED changes or remote control line settings. The logbook task maintains a logbook file, which can be accessed by a user to get information on the history of the system.

\section{Task Configuration}

To provide a useful building block for several chromatographic modules, we had to consider the system integration process. It was obvious that our software development process had to support parallel work on several specific and common tasks. All intertask communication is accomplished by sending and receiving mail. To decouple system integration from the progress of an individual task or of other modules, we took a new approach to task configuration. In contrast to the more traditional close ties between corresponding tasks (i.e., by static tables), we deliberately tried to decouple the individual tasks as much as possible. Thus we came up with an automatic mail configuration process.

At start-up, each task registers with the operating system to reserve the required resources (e.g., RAM, stack) and get the needed priority. In this process, each task declares its needs with respect to mail. A task may contain several mail producers and consumers. These are announced to the operating system. As the mail manager is informed about the type of mail to be received or sent it performs two main services: it generates mail routes leading from each producer to all mail consumers interested in the same sort of message, and it assigns an appropriate number of buffers to each producer. This guarantees availability of the requested resources to each producer better than a common pool of buffers, which could be easily emptied by a single producer.

After initialization the multitasking starts and the tasks compete for CPU time. Our multitasking is not time-sliced, so a task continues to operate until a higher-priority task is scheduled or it suspends itself waiting for mail. A more important task is only scheduled once it receives mail, either from an interrupt-based scanner or from the currently executing task. The executing task thus gets interrupted and suspended while the higher-priority task is awakened with mail received. Thus we use a mail-driven multitasking system.

\section{Mail Management}

The smart mail management system handles all kind of situations: producers without consumers, consumers without any producers, several producers for a given type of mail, multiple consumers for some mail, consumers of several different types of mail, and producers of multiple mail types (see Fig. 6). This built-in flexibility allows easy addition and integration of a new task without modifying tables. If a consumer never receives mail, it is not bothered and never awakened. In contrast, a producer's mail that has no consumer is simply short-circuited back to the producer.

This flexibility made it possible to have early working prototypes by, for example, defining returned mail to be an acknowledgment although no one had ever received that mail. It also allowed easy adding of functionality. For example, assume we want to trace what keys are pressed. We simply write a task that registers for key mail as a consumer. It will automatically receive all key mail, and its only responsibility is to forward the mail after tracing. One can look at tasks simply as objects to be added to a system, while the system resolves all connectivity and resource issues.

\section{Table-Driven Applications}

As already explained, the objective of writing reusable code led to a firmware system composed largely of generic parts that are tailored to specific uses with the help of tables.

Several ways of building ROM tables were considered: - Pseudoinstructions. The tables are built by defining constants with the help of the compiler or by defining the memory contents with the help of assembler pseudoinstructructions such as

\section{DS.B VALUE \\ DS.W \#PROCESS_XY}

- Cross generation. The tables are generated on a remote computer with the help of tools on that computer (UNIX shell scripts, lex/yacc etc.).

- Generate tables with the help of Pascal. The tables are generated by a Pascal program that runs on the target processor. The program initializes a memory area for its own variables, which will later be used as the table.

In the past, we have used the first method, pseudoinstructions, for presetting memory locations (small tables, structured constants). The pseudoinstruction technique has the advantage that the assembler/linker places the defined bytes and words in the ROM area automatically, whereas with the other two alternatives this must be managed separately after the tables are built. The problem with this approach is that there is no automatic link between the assem-

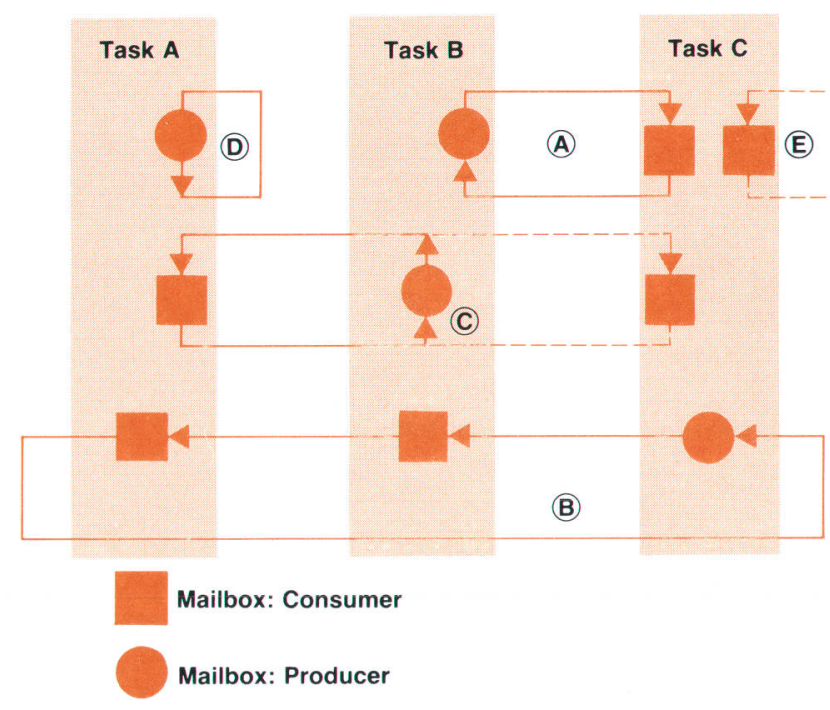

Fig. 6. Producer/consumer configurations for mail. A: one producer, one consumer. B: one producer, several consumers. C: one producer sending mail on different mail routes. $D$ : one producer, no consumer for this type of mail. E: consumer for mail that is never produced. 
bler statements and the data structure of the final table. There is no automatic way of detecting errors in those tables once they are written. Typically the errors show up later at run time. With this experience in mind we rejected this alternative.

The second alternative, cross generation, has the potential of a powerful computer with all of its tools, but still has the disadvantage of not having a link between the data structure of the cross-generated table and the data structure of the programs that later interpret that table.

The third alternative has all the disadvantages of a (typical) target environment, including reduced memory space, no or poor operating system, and no tools. On the other hand, there are some important advantages. First, the procedures building the table use the same data structure (Pascal type declaration) as the interpreting procedures. Second, the table generation process can use the features of the Pascal programming environment, including the compiler (type checks). Because of these advantages, this alternative was chosen.

\section{Table Generation}

Every generic Pascal procedure that has to be customized by a predefined table has to provide a set of Pascal procedures that can be used to build up the table. When generating the tables, the parameters passed to these procedures and the sequence of the procedure calls determine the contents of the table. This process of table generation has to be done once before calling the generic procedure, for example during the start-up phase in the emulator.

Typically the code for table generation is much bigger than the amount of memory used for the final table. Therefore, the table generation code is excluded from the final instrument firmware. This is achieved by burning the tables into ROM after a "protostart" program execution in which only the table generation code is executed (see Fig. 7). This burning into ROM is done together with the step of burning the whole firmware.

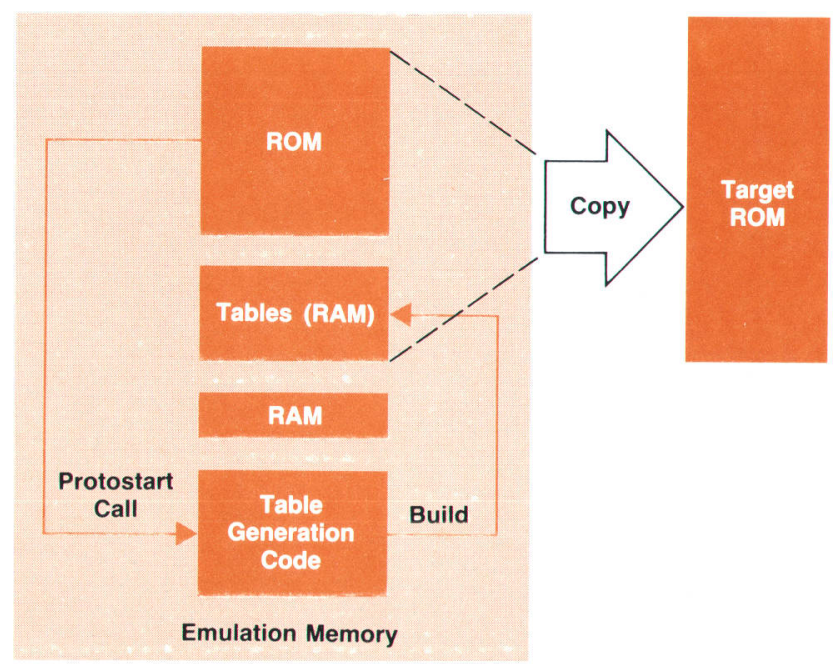

Fig. 7. Generic firmware modules are customized by instrument-specific tables. The tables are generated and burned into ROM along with the other firmware, but to save space, the table-generation code is not placed in ROM.
The following is an example of table generation from the parser table generation process:

PROCEDURE set_cmd_table;

BEGIN

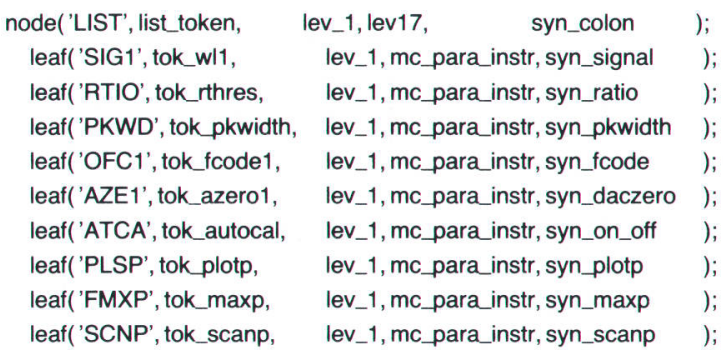

END $\{$ set_cmd_table $\}$;

Node and leaf are Pascal procedures that are called to build up the parser tables. The parameters passed to these procedures are instruction keywords, syntax descriptors and internal tokens for them, and the mail routes to the execution tasks.

\section{Results}

Fig. 8 shows the amount of code written for the HP 1050 modules in thousands of noncomment source statements (KNCSS). More than half of the code $(60 \%)$ is common to all three modules. This common code is tailored to the special needs of the modules by tables which are generated by about $15 \%$ of the code. The rest of the code $(25 \%)$ is module-specific.

Almost all of the firmware is written in Pascal. Assembly
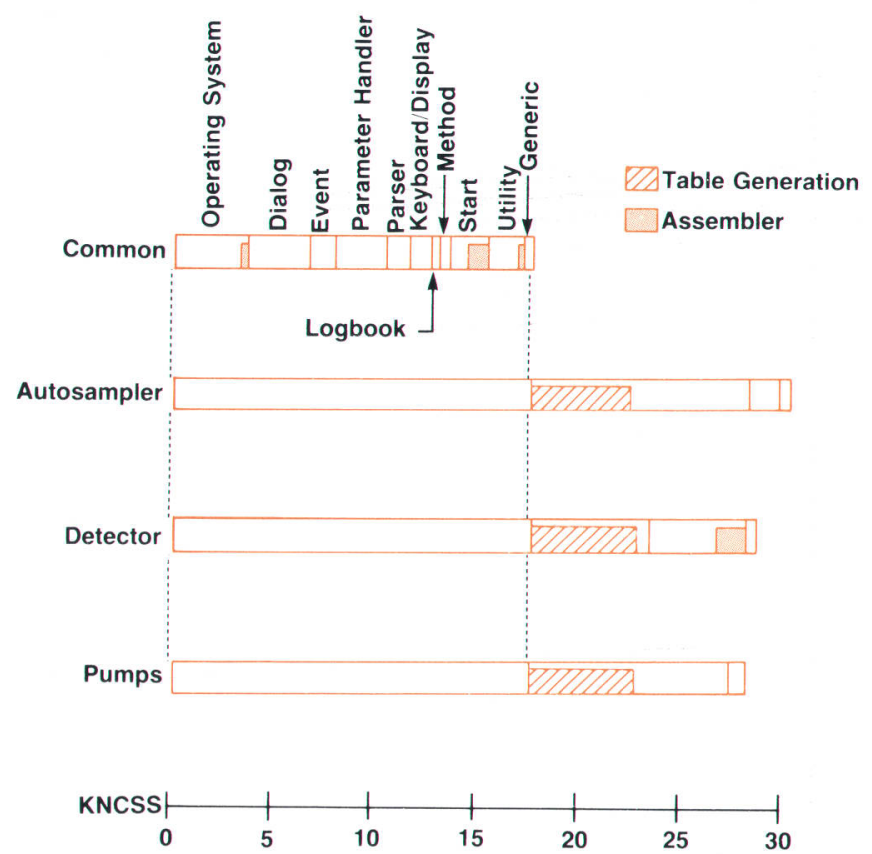

Fig. 8. Distribution of HP 1050 Series firmware in KNCSS (thousands of noncomment source statements). 
language is only used where necessary, for example in the operating system for stack pointer manipulations and interrupt handling or for performance reasons like the RAM test during the start-up phase and data acquisition in the detector.

Strict use of the revision control system (RCS) helped us manage the potential problems of incompatible versions of up to 20 tasks. Automatic task configuration and the automatic include and compile-if-necessary mechanisms helped the firmware engineers change or add tasks without knowing about the complete system in detail.The high level of common code gave us:

- Three times as much test time on the common firmware parts as on instrument-specific parts. Therefore, the common firmware parts turned out to be very robust.

- Common understanding and knowledge of the firmware in each instrument on the part of all firmware engineers working on that project. This put us in a position to balance manpower among the teams working on a specific instrument.

- Early prototypes for both hardware and user interface tests as a result of concentration of effort on the common firmware parts at the very beginning.

- The basis for future enhancements that are common to all instruments (e.g., communication interfaces).

- The basis for new instruments to be developed.

In the future, the extensive use of tables will allow us to enhance the functionality of the instruments incrementally, or to modify, for example, the user interface without altering the common firmware parts. Our experience has shown that modifying the tables is almost always bug-free the first time. Also, improvements of the common firmware parts in terms of performance or functionality always count three times.

\section{Acknowledgments}

The success of this exacting firmware project is the result of good teamwork by the programmers and the support of the hardware engineers. The authors want to thank in particular Herbert Anderer, Fritz Bek, Volker Brombacher, Guenter Hoeschele, Michael Leiber, Klaus Weber, and Klaus Witt for their valuable contributions in programming and in team discussions. Also, many thanks to Claus Lueth, who had to deal with many proposals and complaints while designing and implementing the common processing hardware. 


\title{
HP OpenView Network Management
}

\author{
HP OpenView is HP's first set of integrated hardware and \\ software products designed to address the needs of \\ managing open, standards-based, multivendor networks \\ in a consistent, user-friendly manner.
}

\section{by Anthony S. Ridolfo}

N

ETWORK OPERATORS RUNNING a large computer center are often confronted with questions like the following:

Why can't I connect to the system?

How come response is so slow?

What's wrong with the electronic mail system?

What computers can I connect to from my terminal?

Network managers are also confronted with questions dealing with network planning, inventory control, and accounting. Some typical network management questions include:

What is the overall load on the network?

Which systems are bottlenecks in my network?

How can I plan for new components in my network?

What equipment do I really have, and where?

As departments, sites, and whole companies (referred to as “enterprises") integrate and interconnect their data processing tasks, the job of managing these complex networks of computer and data communications equipment becomes increasingly more important to a company's bottom line. Monitoring the health of a network, diagnosing problems as quickly as possible, controlling the individual components, adjusting network-wide parameters and configurations, accounting for use of network resources, and planning for future expansion are the objectives of network management applications.

These tasks would be relatively simple if all the components in a network came from the same vendor. However, it is indeed rare that all links in a network such as modems, modem cables, multiplexers, pads, LAN connectors, LAN cards, LAN cable types, hubs, bridges, servers, gateways, and PBXs come from the same vendor. There are also different personal computers, workstations, minicomputers, and mainframes. And there are different wide area point-topoint networks, public and private X.25 packet switching networks, and a company's enterprise-wide networks that may even link to their suppliers' and distributors' networks.

The computer industry and international organizations such as ISO (International Organization for Standardization) recognize the need to standardize communication and network management protocols. It is HP's stated objective to be the leader in open, standards-based, multivendor networking. This includes the capability to manage and be managed by standards-compliant applications running on equipment supplied by any vendor.

However, having applications that can access and control a network is not enough to address the needs of network managers and network operators. Each type of equipment to be managed has its own particular command interface and management capabilities. Therefore, a unifying, consistent, user-friendly interface is needed for these applications.

\section{HP OpenView}

The HP OpenView network management family is designed to address these needs of managing open, standardsbased, multivendor networks in an open, consistent, userfriendly manner. The HP OpenView products provide a consistent user interface and an integrated environment for monitoring, diagnosing, controlling, and measuring the performance of network components. From a single display, a network operator can see a graphical representation of the network components and their interrelationships, make configuration changes, and run diagnostic and performance gathering applications.

The design philosophy for the HP OpenView products is to create for the end user an easy to learn, easy to remember paradigm for accessing sophisticated network management applications. HP OpenView Windows, which is the HP OpenView product that provides the user interface, runs on an HP Vectra ES/12 personal computer as a MicroSoft ${ }^{\circledR}$ Windows application. Each of the HP OpenView products, in turn, runs under HP OpenView Windows. The user first runs the HP OpenView Windows draw (OVDraw) program that allows the user to draw a map of the network, with specific icons representing network devices, such as an HP 3000 business computer or a bridge (see Fig 1). The user then saves this map, and runs the HP OpenView Windows run-time program (OVRun). The map becomes a dynamic, graphical shell for accessing the applications running under HP OpenView Windows. In addition, if there exists an application reporting the state of a device to the HP OpenView system, the current operational health of the device is indicated by the color of the specific icon (e.g., red for critical, yellow for warning, and green for $\mathrm{OK})$.

Suppose the network operator wishes to set some parameters on a specific bridge on the network. The operator would click the mouse on the symbol representing the specific bridge and select the menu item Set Parameters.... This selection causes HP OpenView Windows to pass program control to the HP OpenView BridgeManager software, which puts up a dialog box for this function. Suppose now the operator needs to set parameters on a specific HP 3000

Microsoft is a U.S. registered trademark of Microsoft Corp 
datacom and terminal controller (DTC). Again, the operator selects the specific icon and then the Set Parameters... menu item. Program control now passes to the HP OpenView DTC manager, which puts up the relevant dialog box for this function. At no time does the operator need to know which product to invoke, or how to invoke it. HP OpenView Windows and the graphical shell perform this function by controlling what menu items are enabled for which devices or icons. The user is relieved of the need to know what actions are legal or illegal for a particular type of device. The article on page 60 describes HP OpenView Windows in more detail.

This type of intelligent user interface is important in network management because network operators usually do tests as a response to an alert of some kind, such as an icon changing color on the display. They do not perform testing and diagnostic functions all the time, or even on the same equipment. It is therefore important to maximize the familiarity of the user interface as well as to minimize the number of ways the user invokes functions to do similar tasks. HP OpenView Windows and its supporting applications help with this task by providing a context sensitive, interactive system for guidance.

\section{Network Management Applications}

The HP OpenView articles in this issue describe the development efforts and the underlying architecture of the initial set of network management products in the HP OpenView family. These products include:

- HP OpenView Windows. This product provides the user interface to network management applications and a set of utilities that enable developers to create network management applications to run in the HP OpenView Windows environment. HP OpenView Windows is divided into two main parts: the end user run-time product and the HP OpenView Windows developer's kit. The end user run-time product includes the hardware and software required to use HP OpenView Windows. The developer's kit includes the end user run-time product and the necessary libraries, include files, sample source code, and documentation to facilitate the developer's task of creating a Microsoft Windows application that can be integrated into the HP OpenView end user run-time product. The HP OpenView developer's kit includes a style guide for helping developers ensure that the detailed look and feel of their dialog boxes are consistent with other, independently developed applications.

" HP OpenView Windows BridgeManager. This product provides centralized monitoring and control of HP 28648B 10-Mbit/s LAN Bridges and HP 28647B StarLAN Bridges (revision B bridges only). Using the network map feature of HP OpenView Windows, bridges can be easily labeled and identified. Menus allow quick access to a variety of bridge management features, including configuration, monitoring, control, performance management, and problem identification. The HP OpenView BridgeManager is described on page 66.

- HP OpenView Data Line Monitor. This product provides the ability to monitor 4-wire leased point-to-point analog data lines using an HP 4948A In-Service Transmission Impairment Measuring Set controlled from the HP OpenView workstation. It is a network monitoring system that can measure performance and aid fault isolation during troubleshooting while the network is being used. The HP OpenView Data Line Monitor is described on page 71.

- HP Open View DTC Manager. This product provides centralized and integrated network management for both terminal connectivity and X.25 networking. It configures, monitors, diagnoses, controls, and downloads software to DTCs (datacom and terminal controllers). From a single HP Vectra running HP OpenView Windows and the DTC Manager, an operator can manage local and

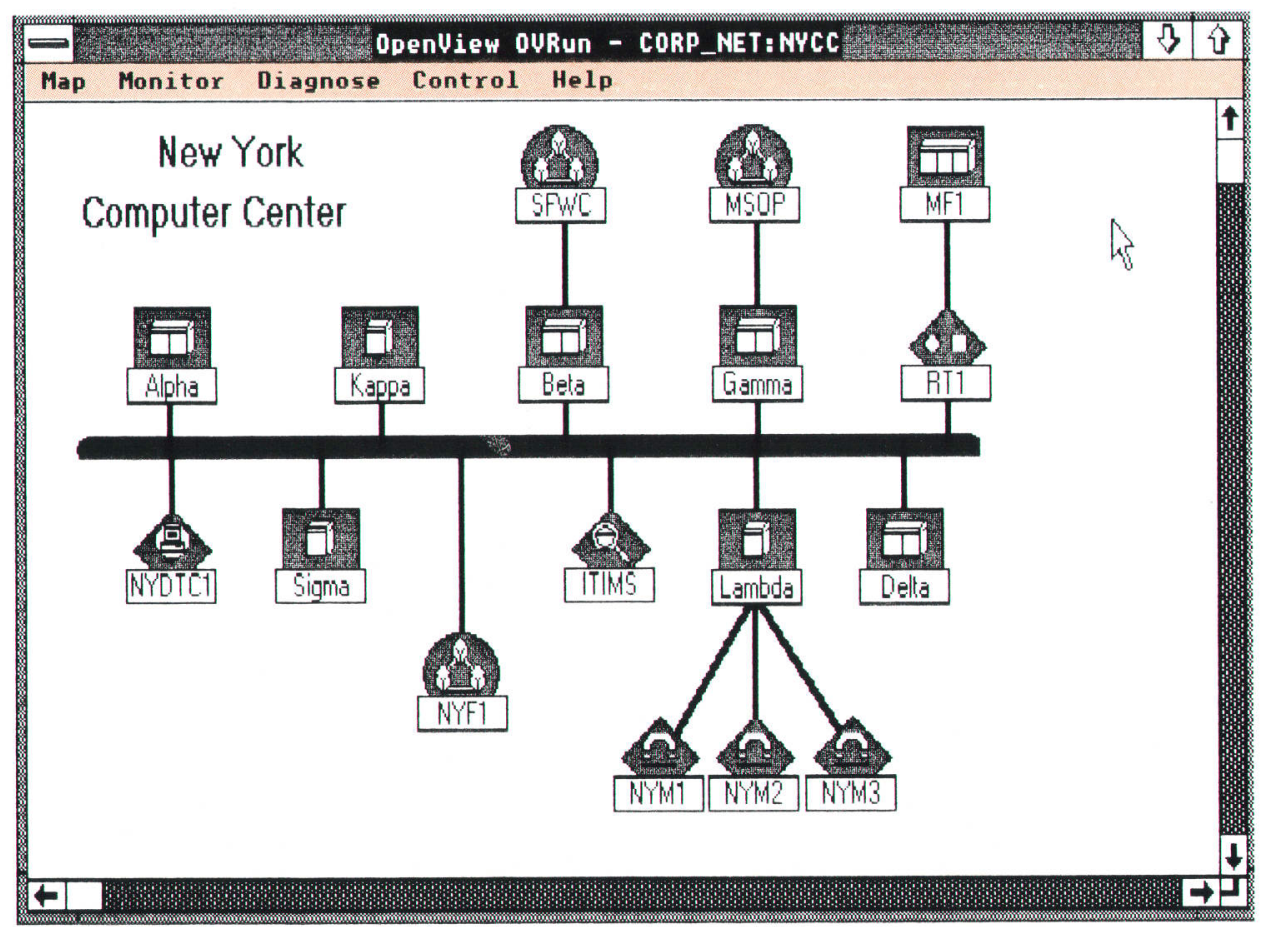

Fig. 1. An HP OpenView Windows map showing the network configuration for a computer center. 
remote terminal connections and remote system-to-system communications across multiple DTCs on a LAN The HP OpenView DTC Manager is described on page 76.

- HP OpenView NS Monitor. This is an internal HP tool developed to test and refine the HP OpenView architecture and the facilities provided by the HP OpenView Windows software. It is not available as a product. It provides network management services in the form of centralized or distributed network status, diagnostic, and performance monitoring for HP 3000 computers running the MPE V operating system. The user interface software runs on an HP Vectra personal computer under HP OpenView Windows, and the programs that perform the diagnostic and performance monitoring run on an HP 3000 computer that is designated as the management node. The systems being managed are called managed nodes. The user interface software on the Vectra and the programs on the management node communicate with each other via HP OfficeShare on the Vectra and NetIPC on the HP 3000. These products are described on page 85 .

\section{Conclusion}

A direct result of the HP OpenView design philosophy is that application developers have to learn to split the functionality of their products between the network management functions and the user interface code. This is in alignment with HP's NewWave philosophy and the vision of truly distributed applications. Although network management products address a very specific end user, the architecture and implementations used to develop the products mentioned above represent the future of computer applications. The article on page 54 describes the HP OpenView network management architecture that provides the models to guide the planning, analysis, and design of network management products developed to run in the HP OpenView Windows environment.

\section{Acknowledgments}

The development of the initial set of HP OpenView products was the combined efforts of teams at HP's Information Networks Division (IND), Roseville Networks Division (RND), Grenoble Networks Division (GND), Colorado Networks Division, and Queensferry Telecommunications Division (QTD). I thank all the engineers and managers at these organizations who contributed to the HP OpenView product family. Specific thanks go to the following individuals: Larry Goldman, CND section manager, Alan Pare, IND distributed applications lab manager, Catherine Smith, IND project manager for HP OpenView Windows, Atul Garg, IND project manager for the HP OpenView diagnostic and performance monitors, Shnider Youssef, IND project manager for the HP OpenView core services, Phill Magnuson, RND project manager for the HP OpenView BridgeManager, Mike Hurst, QTD project manager for the HP OpenView Data Line Monitor, Bernard Guidon, HP Information Networks Group marketing manager, and Jean-Jacques Ozill, GND project manager for the HP OpenView DTC manager. 


\title{
HP OpenView Network Management Architecture
}

This article highlights the principal objectives of the architecture and the reference models used to support

the HP OpenView product development.

\author{
by Keith S. Klemba, Mark L. Hoerth, Hui-Lin Lim, and Maureen C. Mellon
}

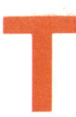

HE USE OF INFORMATION NETWORKS in commercial, government, and academic organizations has exploded in the 1980s. With wide and local area networks, computing power has migrated from a centralized to a distributed environment. This has reduced costs, enhanced competitiveness, and renewed organizational creativity. The explosion in the number of networks and network devices from a variety of vendors has caused a dramatic increase in network complexity and an acute need to manage these distributed resources more effectively.

The need for network management is apparent today throughout any organization deploying networks. The en- terprise network in use by many organizations includes both local and wide area networking technologies and an assortment of network devices from different vendors, and is managed by a team of professionals in different geographic areas. Fig. 1 shows the domain of a typical enterprise network.

Companies are now using information management to gain a competitive advantage by delivering goods and services faster and more efficiently. While the benefits of network management are most apparent during a crisis, they are no less important in day-to-day network operation to control network faults, configurations, performance, or se-

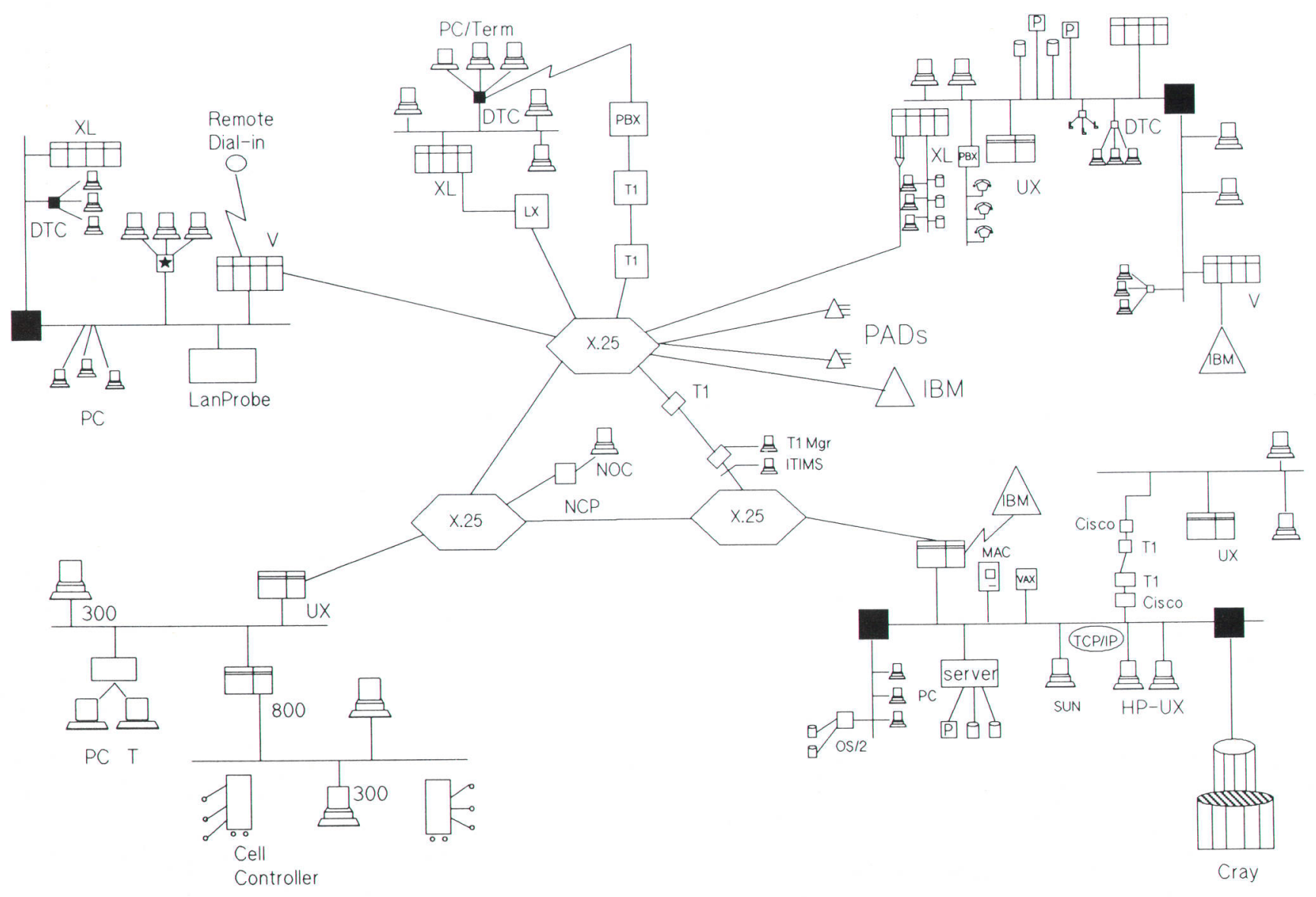

Fig. 1. Typical enterprise network includes both local and wide area netorking technologies and an assortment of network devices from different vendors. 
curity. An integrated network management system can save time and money by reducing downtime and performance problems.

\section{Problems of Stand-Alone Element Management}

During the last decade, most of the computer and networking industry focused on the rapid evolution of network technology to the neglect of network management products. As the need for network management became apparent, the first products made available were stand-alone element managers. These products manage a particular set of network elements, such as bridges, modems, or routers. As more devices are included in the network, the network manager must add more stand-alone element managers. With no correlation, aggregation, or prioritization of information across applications or consoles, it quickly becomes impossible for the network manager to use this large number of element managers effectively. Typically, each element manager uses a different user interface and style of operation, so that different training and expertise are required for each system.

In contrast, an integrated network management system like HP OpenView combines and consolidates the management of network elements from various vendors. It allows the customer to monitor, control, automate, and repair network segments and equipment from a single console and user interface.

\section{Dimensions of Integrated Network Management}

The problem of managing the enterprise network can be divided into three components: the users of network management, the objects to be managed, and the functional needs of network administrators. Each component influences network management architecture by imposing requirements for effective management.

Users. There are several users of network management. The corporate network manager works at the executive level and is concerned about network costs, uptime, and strategic planning. The main objectives are to control the network asset and obtain consistent, maximal performance. Telecommunications and MIS directors implement and operate large portions of corporate wide area networks. These managers are concerned about network growth, uptime, and planning. At the local area network level, data communications specialists, system operators, and site telecommunications managers oversee work-group networks in environments ranging from engineering research and development to manufacturing plants to business offices. Users of local area networks rely on their managers to maintain the connection into the local EDP environment, provide assistance with personal computer software management, and keep the network operating during critical periods.

Resources to Be Managed. These fall into four categories or layers, as shown in Fig. 2. The transmission layer, which corresponds to the first layer in the ISO OSI model, includes the physical media, such as T1 multiplexers, modems, broadband cable, fiber optic cable, and the like. The data network layer, which includes transports and services, covers LANs, X.25 and SNA networks, and OSI services. A growing number of customers are adding voice elements to this category. The computation network layer includes networked systems and networked data bases. The networked applications layer consists of distributed applications in areas such as X.400 electronic mail, electronic data interchange, and office automation.

Functional Requirements. HP defines several specific functional areas for network and system management. Fault management provides the ability to identify, diagnose, and resolve network problems quickly. It also includes status monitoring, alarms and alerts, and predictive expert system tools. Configuration management delivers the ability to track network and device configurations from a central control point. Performance management provides the ability to optimize network performance through the collection and analysis of device performance data. Accounting provides the network manager with network use information. Security protects the network and its components from unauthorized intrusion or surveillance. Inventory management addresses the need to track, monitor, and maintain assets over a wide geographic area.

The foundation for integrated network management is vendor support and service. Faced with budget constraints and a shortage of skilled staff, network managers need planning, implementation, and operation support. Consulting, training and support services can speed the implementation of integrated network management and ensure its effective use.

\section{A Standards-Based Architecture}

The HP OpenView network management architecture (NMA) is rooted in international and de facto industry standards. ${ }^{1,2,3}$ HP OpenView NMA is based on the Open Systems Interconnection (OSI) management framework and models developed by the International Organization for Standardization (ISO). Standards provide HP OpenView NMA with a solid foundation for providing interoperability in heterogenous, multivendor enterprise networks by defining network management protocols and mechanisms for sharing management information. As a result, products based on HP OpenView NMA can be used to manage any type of network that conforms to the OSI standards. HP OpenView NMA derives three essential elements from the OSI standards on which it is based: a network management framework, a well-defined mechanism for describing managed objects, and a set of services and protocols for communication.

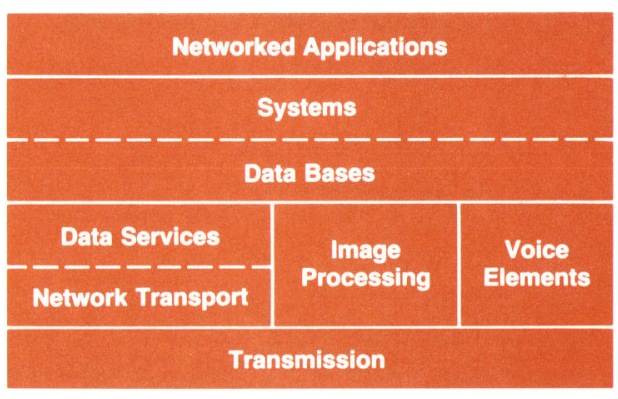

Fig. 2. Network resources to be managed fall into four layers 


\section{OSI System Management Model}

In 1988, the ISO specified the OSI System Management Model as a basic framework for network management. In the OSI model, a manager process is responsible for realizing the specific management functions requested by the user through interactions with agent processes. The agent processes represent the management services offered by managed objects. ${ }^{4}$ Possible management services include:

- Operations such as reading a counter

- Actions such as resetting a network device

- Notifications (known as events) such as an indication that a threshold has been exceeded.

Fig. 3 illustrates the OSI System Management Model. The manager process and the agent process use a Common Management Information Protocol (CMIP) to exchange management information. ${ }^{5}$ The interface labeled $\mathrm{A}$ is the only open integration point in the OSI System Management Model. The management services offered by managed objects at this interface are defined through the use of a standard specification scheme known as the OSI Structure of Management Information. ${ }^{6}$ The interface labeled B is an unspecified integration point that can be implemented using industry-standard or proprietary protocols.

\section{OSI Object Model}

The Structure of Management Information is a set of rules or guidelines for defining classes of managed objects. The rules are used when defining each class of managed object to ensure specification uniformity Classes of managed objects can be defined for any manageable network resource. For example, there can be a managed object class that describes LAN bridges, another that describes computer systems, and a third that describes network equipment in general.

Object classes are defined by specifying their attributes, operations, actions, and events. Once an object is defined, it is placed within a registration hierarchy and a unique class identifier is assigned by the registration authority. For example, a managed object describing a piece of network equipment might have:

- Attributes detailing its physical location, state, and percentage of utilization

- Actions to request its activation or deactivation

- Events such as alarm and change reporting.

The use of an object model is important because it brings with it the concept of inheritance. In the context of network management, inheritance allows refinement of existing classes while ensuring compatibility with existing software. The inheritance relationship that exists between object classes is important because it allows existing management applications to work with the new object class, and it provides a mechanism for software reuse.

The set of managed objects within a system constitutes that system's Management Information Base. ${ }^{2}$ Instances of managed objects exist within a containment hierarchy referred to as a containment tree. For example, a real open system (computer) would contain numerous managed objects such as a routing table, which would contain entries, or an n-layer protocol, which would contain n-layer connections. Some of the benefits associated with the containment relationship are:
- If a managed object is deleted, all managed objects contained within it are also deleted.

- Management requests can be directed to a group of objects related by containment using scoping.

\section{Beyond the OSI Standards}

HP OpenView NMA specifies a complete environment of services and facilities available to management applications distributed throughout the network. This vision of HP OpenView NMA requires the addition of architectural components beyond those described in the OSI System Management Model.

The HP OpenView NMA reference model, illustrated in Fig. 4, has nine components. It refines the OSI model with three major extensions, which are essential to the management of complex, multivendor enterprise networks. First, HP OpenView NMA creates a distributed network management communication infrastructure consisting of three components known as supervisor (S), postmaster (P), and communication profiles (C). Second, HP OpenView NMA adds an additional point of integration by dividing the manager process into a management application (A) and a user interface (U). Third, HP OpenView NMA extends the object-oriented paradigm in two ways. The need for managed object persistence is addressed with the specification of managed object data stores (D). The scheme used to define managed objects $(\mathrm{O})$ is also used to specify key application functionality in the manager process as a managed nbject, making the management services (M) it provides available for reuse. This provides another open point of integration. Finally, environmental services (E) are the services provided by the native operating system and these can be used by any of the other components.

\section{HP OpenView Object Model}

u S O Object-oriented concepts and technology A $\mathbf{P} \mathbf{M}$ architecture reduces the multidimensional E C D problem illustrated in Fig. 1 to a single dimension by using a common object model for describing all resources to be managed. HP OpenView NMA uses the concepts defined by the OSI standard for describing managed objects. HP OpenView NMA supports the structures of management information used by the OSI model and the Internet Engineering Task Force (IETF). HP OpenView NMA also provides support for managed objects defined in the ISO and IETF management information bases and is expandable to include objects defined by developers for special purposes.

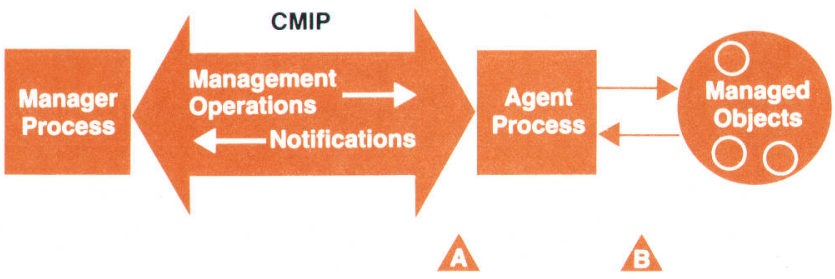

CMIP = Common Management Information Protocol

Fig. 3. OSI System Management Model. 
Managed objects are an abstraction of the real resources being managed. The management services (M) offered by a managed object consist of the software (programs) necessary to provide the services defined in the managed object specification. This software may contain several parts, such as the infrastructure interface, an object manager, and environmental services (E) necessary for communication with the real resource.

HP OpenView NMA extends the OSI object model by describing managed object data stores. These are identical to managed objects except that they are persistent. In the OSI model, managed objects are volatile. The managed object data stores model a mechanism for maintaining information about managed objects even when networks or systems are powered down.

\section{Distributed Communications Infrastructure}

\begin{tabular}{|c|c|c|}
\hline U & S & O \\
\hline A & P & M \\
\hline E & C & D \\
\hline
\end{tabular}

Network management is a distributed activity in that the user interfaces, management applications, and management services can be located in different systems throughout a network. The HP OpenView NMA communications infrastructure (Fig. 5) provides the facilities for establishing and maintaining communication between these components. The communications infrastructure consists of the postmaster (P), the supervisor (S), and communications profiles (C). These components draw heavily upon the environmental services (E) to carry out their functions.

The postmaster (P) provides basic message routing services between the network management components listed above. It operates as a table-based object-oriented message router. The routing table shown in Fig. 5 is the focal point of the postmaster's functionality and is itself a managed object. Given a message (perhaps from an application) addressed to a specific managed object, the postmaster looks up the managed object name in the routing table to find the information necessary to deliver the message to the managed object. The fields in the postmaster's routing table provide the managed object name, the communication profile number, and profile-specific data.

The information required to perform the name-to-address translation can be obtained from a directory service. The postmaster can make use of the directory service through the supervisor, which has the responsibility for creating and maintaining the routing table. However, the postmaster must be able to operate independently in case these supporting services fail. The routing table can be considered a cache of information derived from directory services and other sources.

The supervisor (S) administers the existence of and access to all the components associated with the communications infrastructure. It has the authority and the ability to initiate, cancel, lock and unlock, and control access to management services within its supervisory domain.

The HP OpenView NMA process of exchanging management information is adopted from the OSI framework. An application layer network management protocol supports transaction-oriented exchanges between the distributed processes. HP OpenView NMA supports formal and de facto industry-standard protocols with a common network management communications application program interface based on the OSI Common Management Information Services (CMIS). ${ }^{7}$ In an OSI environment, CMIP (as shown in Fig. 5) would be the network management protocol of choice. In an Internet TCP/IP environment, the most widely used protocols are the Simple Network Management Protocol (SNMP, specified in RFC 1098) and CMIP over TCP/IP (CMOT, specified in RFC 1095). HP OpenView NMA supports not only these network management protocols but also the addition of proprietary protocols under the common application program interface for complete integration in a multivendor, heterogenous network. Each protocol stack is modeled as a communications profile managed object; this allows extensibility of the communications infrastructure illustrated in Fig. 5.

The environmental services (E) represent the facilities provided by the environment in which the network management solution must operate (e.g., HP-UX, MPE, MSDOS). The capabilities provided by these environments can be used by any of the components of HP OpenView NMA. In this context, these services could include the file system, the X.500 naming system, or proprietary network management protocols. It is also possible that some of these services could become part of a managed object. For example, a managed object that is responsible for report generation and delivery service would collate the information to be presented in the report, create a file, and deliver it to any requesting application using a file transfer environmental service such as FTAM (File, Transfer, Access, and Management).

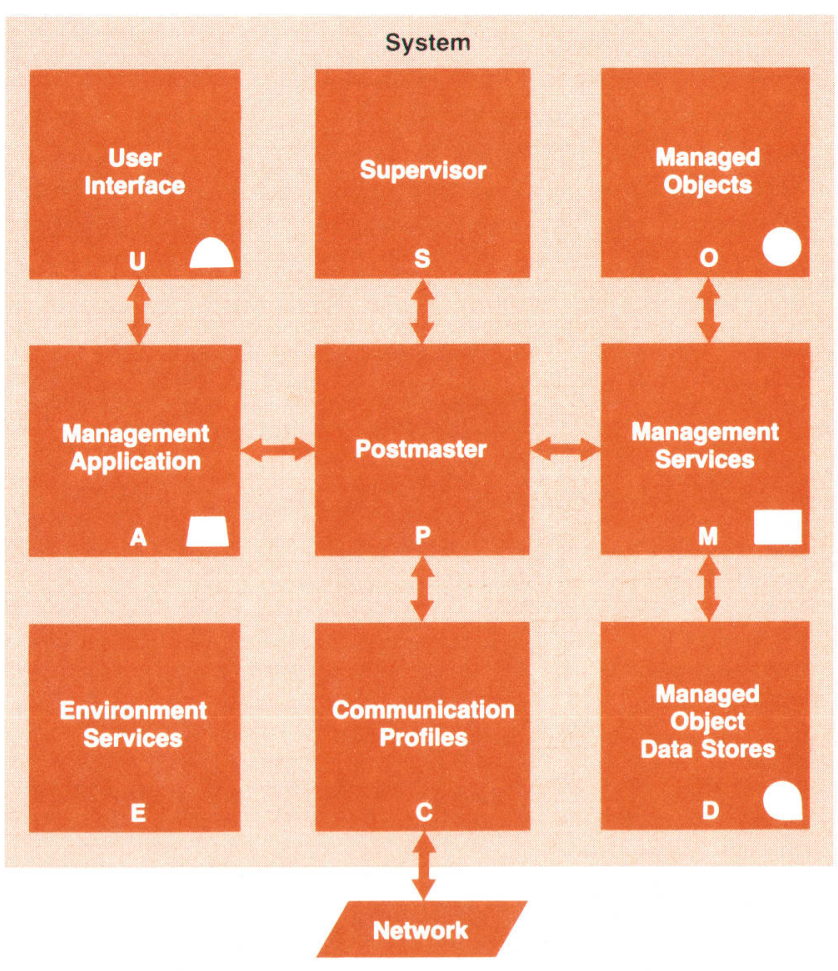

Fig. 4. Components of the HP OpenView network management architecture reference model. 


\section{Multiple User Interfaces}

- HP OpenView NMA specifies the user interU S O face as a separate component of the network A $\mathbf{P} \quad \mathbf{M}$ management solution. This addresses the E C D need to allow users to access network managewhich may include dumb terminals, workstations with graphic displays, voice, or any appropriate devices. It also addresses the need to be able to build solutions that meet individual users' requirements so that only relevant information is provided.

The separation of the user interface from the management application provides an additional point of integration so that several management applications can drive a single display or several displays can be driven by a single management application. User interfaces can be distributed throughout the network using environmental services (E) to access remote management applications (A).

\section{Extension of the Object-Oriented Paradigm}

$\mathbf{U}$ S 0 HP OpenView NMA extends the power of S $O$ the object-oriented paradigm as specified in A $\quad \mathbf{P} \quad \mathbf{M}$ the OSI model by treating portions of applicaE $\quad$ C $\quad$ D tion functionality as managed objects. These managed objects are defined using the same object specification techniques described previously. The result of this approach is that value-added application functionality that was previously only available within a given application now becomes available as a source of information or services to other management applications. This encourages the full integration of products into a hierarchy of management solutions while reducing duplication of functionality and many concerns about consistency. Thus, what was previously a monolithic application is decomposed into a much reduced management application with an accompanying managed object that offers management services identical to the value-added functionality that was previously locked within the application.

The additional point of integration provided by HP OpenView NMA between management applications and these managed objects also opens functionality for wider use by more applications. Consequently, HP OpenView NMA facilitates the development of applications upon a base of existing managed objects. For example, a traditional fault application would include event processing capabilities. When a configuration application is to be installed in the same system, it would not normally have access to event processing in the fault application, and would have to duplicate that functionality. HP OpenView NMA encourages the specification of event managers as managed objects, thereby making event management services available to other management applications.

These managed objects can provide services not only to management applications but also to other managed objects. HP OpenView NMA describes a scheme in which the most significant value-added portions of a network management solution are described as managed objects, so they can be linked into management chains, providing comprehensive services to management applications.

\section{Conclusion}

It is important to stress that HP OpenView NMA is intended as a reference model for developers of network management products. It is not intended to mandate the implementation of all the components described above. Neither are the components intended to define program or process boundaries. Products implementing HP OpenView NMA may range in complexity from bridges to complete systems. Bridges, for example, may only implement the management services defined for the bridge managed object class along with a specific protocol stack. The remaining components could be distributed on other systems as needed.

Initial HP OpenView products implement portions of HP OpenView NMA. For example, HP OpenView Windows (see article, page 60) provides a rich environment for developing graphic user interfaces customized for network management. Newer HP OpenView products will implement more features of the architecture.

In summary, HP OpenView NMA uses the OSI standards

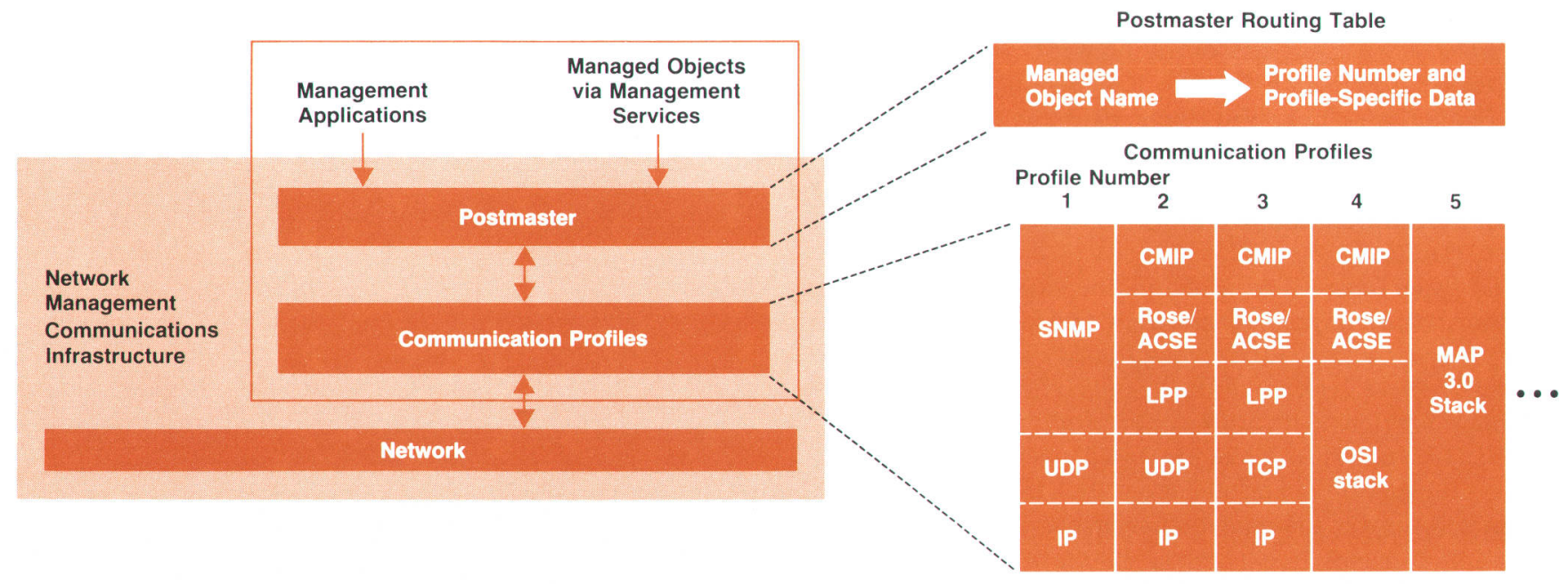

Fig. 5. Distributed communications infrastructure of the HP OpenView network management architecture 
to manage multivendor environments. It refines the standards to introduce additional points where integration of products can take place and by doing so reduces duplication of product functionality. It also allows for customization to support specific network management activities by building on an existing base of managed objects.

\section{References}

1. Information Processing Systems - OSI - Basic Reference Model - Part 4: Management Framework, ISO/IEC 7498-4, 1989.

2. New Recommendations of the "M" Series, AP IX-31-E, International Telegraph and Telephone Consultative Committee (CCITT), IXth Plenary Assembly, Document 31, Study Group IV, Report R26, April 1988.

3. Advanced Networked Systems Architecture (ANSA) Reference Manual, Rel.00.03, ANSA, June 1987

4. Information Processing Systems - OSI - Systems Management Overview, ISO/IEC DP 10040, January 1989.

5. Information Technology - OSI - Common Management Information Protocol Specification, ISO/IEC 9596, 1989.

6. Information Technology - OSI - Structure of Management Information, ISO/IEC DP 10065, 1989.

7. Information Technology - OSI Common Management Information Definition, ISO/IEC 9595, 1989. 


\title{
HP OpenView Windows: A User Interface for Network Management Solutions
}

\author{
HP Openview Windows provides a consistent graphics- \\ based user interface for users of network management \\ applications, and a set of utilities that enable developers \\ to create network management applications for the HP \\ OpenView Windows environment.
}

\section{by Catherine J. Smith, Arthur J. Kulakow, and Kathleen L. Gannon}

P OPENVIEW WINDOWS is a graphical user interface based on the Microsoft Windows environment that provides facilities for handling the user interface for network management applications. For application developers, HP OpenView Windows provides programs to carry out tasks such as drawing a network map or handling alarms. From the end user's perspective. HP OpenView Windows combines the functionality of many of the user's network management applications under one easy-to-use interface, simplifying the learning curve.

This article describes the features provided by HP OpenView Windows to developers and users. Some of the other HP OpenView articles in this issue describe the details of interfacing to HP OpenView Windows.

\section{Overview}

HP OpenView Windows consists of three programs: OVDraw, OVRun, and OVAdmin. These three programs provide the following functionality:

- OVDraw. This program allows users to create maps made up of one or more pictures that represent a data network.

- OVRun. This program provides the facilities that allow users to monitor, diagnose, and control their networks. OVRun uses the maps created with OVDraw.

= OVAdmin. This program is used to set operating characteristics for HP OpenView Windows and HP OpenView applications. These include functions such as assigning passwords and setting up network management parameters.

The graphical user interface consists of maps, pictures, and symbols used to represent a network. A map depicts the whole network and is made up of pictures, each of which shows a portion of the network. For example, a map may represent a network for a group of buildings, while each picture of the map shows the network for one of the buildings. Pictures are composed of symbols that portray

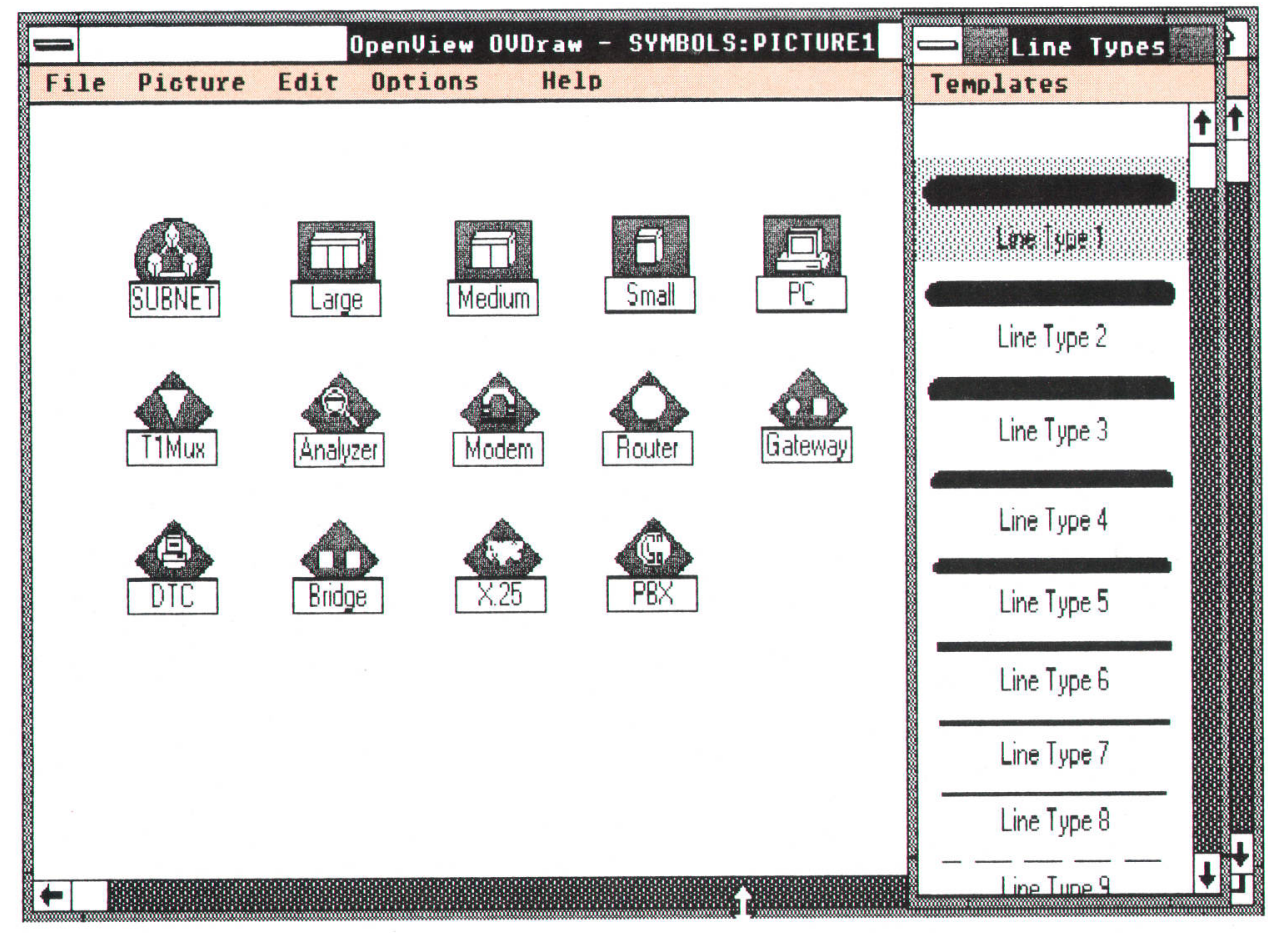

Fig. 1. Some of the symbols used by HP OpenView Windows to represent network components that are managed by network management applications. 
network components, such as computers, modems, bridges, X.25 switches, lines, or a portion of a network (subnet). The symbols provided by HP OpenView Windows are shown in Fig. 1. Subnet symbols are special symbols that are used to signify what is contained in another picture of the network and to help the user navigate around the network. As will be shown later, subnet symbols can be used to represent the network configurations in different locations (e.g., different buildings).

Symbols also provide configuration and status information. Network topology information is provided by the way in which symbols depict connections between components in a network. Status information is provided by the colors of the symbols, which represent the condition or state of each device. Network status is discussed later in the article.

The user gives instructions to HP OpenView Windows and HP OpenView applications via a combination of symbols, menus, and dialog boxes. Symbols represent the network components described above, and they are used to select the network component the user wishes to work on. Menus and menu items represent the operations the user can select to perform network management functions. Menus and menu items may be standard HP OpenView menus or menus added by an application. Dialog boxes allow the user to give instructions to an application. The overall structure and capabilities of dialog boxes are provided by Microsoft Windows. The content of a particular dialog box is provided by the application.

HP OpenView Windows provides a large part of the user interface for applications, but applications must provide part of the user interface. Applications can add to the HP OpenView Windows user interface by creating new menus and adding new menu items, and by adding dialog boxes. The articles on pages 66, 71, and 85 describe adding menus and dialog boxes to HP OpenView Windows.

The HP OpenView Windows product is divided into two categories: end user products and application developer products. The end user products contain the hardware and/ or software components required to use HP OpenView Windows. The software component includes the three programs OVRun, OVAdmin, OVDraw, and one or more HP OpenView applications. The recommended hardware configuration consists of the HP Vectra model ES/12 personal computer with a 40-megabyte hard disk and a 2M-byte expanded memory board. For developers the key product is the HP OpenView Windows Developer's Kit, which contains the HP OpenView Windows end user software and the pieces needed to develop HP OpenView Windows applications.

\section{Application Installation}

When the HP OpenView Windows software is installed on the Vectra, it creates two sections in the Microsoft Windows WIN.INI file. The two sections are called OpenView and OpenViewApps. The OpenView section contains information such as the default network map and the name of the file used for logging. The OpenViewApps section contains entries for HP OpenView applications, which are filled in when applications are installed. An entry in the OpenViewApps section is in the following format:
[OpenViewApps]

AppName $=$ AppRun.Exe,AppDraw.Exe,AppAdmin.Exe

AppName is the application name, AppRun.Exe is an executable file to be started when the end user runs OVRun, AppDraw.Exe is an executable file that is started when OVDraw is run, and AppAdmin.Exe is the executable file started when OVAdmin is run. Applications don't need to have executables for all three HP OpenView programs.

When an HP OpenView Windows application is installed, it is registered with HP OpenView Windows through the entry in the WIN.INI file. HP OpenView Windows applications also register for graphic symbols and menu items. Registration for symbols (or objects in Microsoft Windows terminology) and menus is accomplished by calls to HP OpenView Windows intrinsics.

When one of the HP OpenView Windows programs (OVRun, OVDraw, or OVAdmin) is started, HP OpenView Windows checks the WIN.INI file and invokes all the HP OpenView applications installed there. When an application is invoked, its WinMain loop is entered and the first HP OpenView Windows intrinsic called is OVInit(), which sets up communications between the application and HP OpenView Windows. The application then calls OVRegister( ) to inform HP OpenView Windows what object types (symbols) the application manages, and OVMenuAdd( ) and OVMenuAddltem( ) to inform HP OpenView Windows which menus and menu items are valid for the given object types. The application informs HP OpenView Windows that the initialization is done by calling OVInitComplete( ).

Four main facilities are provided by HP OpenView Windows: map handling, menu integration, status, and context sensitive help.

\section{Map Handling}

When we started considering the requirements for a user interface for network management applications, it was obvious there was a need for a graphics-based user interface. Typically the way network managers and operators use a network management application is to draw a picture of the network on paper, annotating the drawing with identification and other information. The data from the drawing is used to tell the network management application which network components to manage.

HP OpenView Windows simplifies this task by supplying the network map composed of graphics symbols that represent the network components. Associated with each symbol is a network management application that is invoked when the particular symbol and a menu item are selected. Thus, the network manager no longer needs to run an application and then identify the network component of interest. Also, when a network manager is monitoring a network and the state of some network component changes, it is much faster for the network operator to identify the node having problems by looking at a network topology rather than having to look up a node name or address.

In Fig. 2 the user wants to identify a computer system Delta (i.e., get details on the machine type, version number, etc.), and so selects the computer symbol labeled Delta and the Identify menu item under the monitor menu. After the user selects * the Identify menu item, HP OpenView Windows *Selection is accomplished by clicking the left mouse button on a symbol or menu item. 
passes the information to the application registered for that symbol type/menu item pair. The application then has control, and can use HP OpenView Windows routines to access the device or stored data to accomplish the actions associated with Identify. In the example in Fig. 2, the application registered for the Delta computer system is NS Monitor, which is listed at the top of the dialog box in Fig. 2b. If the user wanted to identify a bridge, a bridge symbol and then the Identify menu item would be selected. The article on page 66 describes how the HP OpenView BridgeManager implements the Identify function.
The map can be organized in any way the customer wants to view the network: physical, geographical or organizational. For example, suppose a company has four computer systems - two in building 1 and two in building 2, and the two sites are connected via a LAN bridge. The user can draw a hierarchical map (Fig. 3) or a network model map (Fig. 4). These examples also demonstrate that the user is not limited to placing a symbol in only one picture. In Fig. 4 the subnet symbols BLDG1B and BLDG2B and the bridge symbol BRIDGB show up in more than one display. BRIDGB represents the same physical bridge each time it appears.

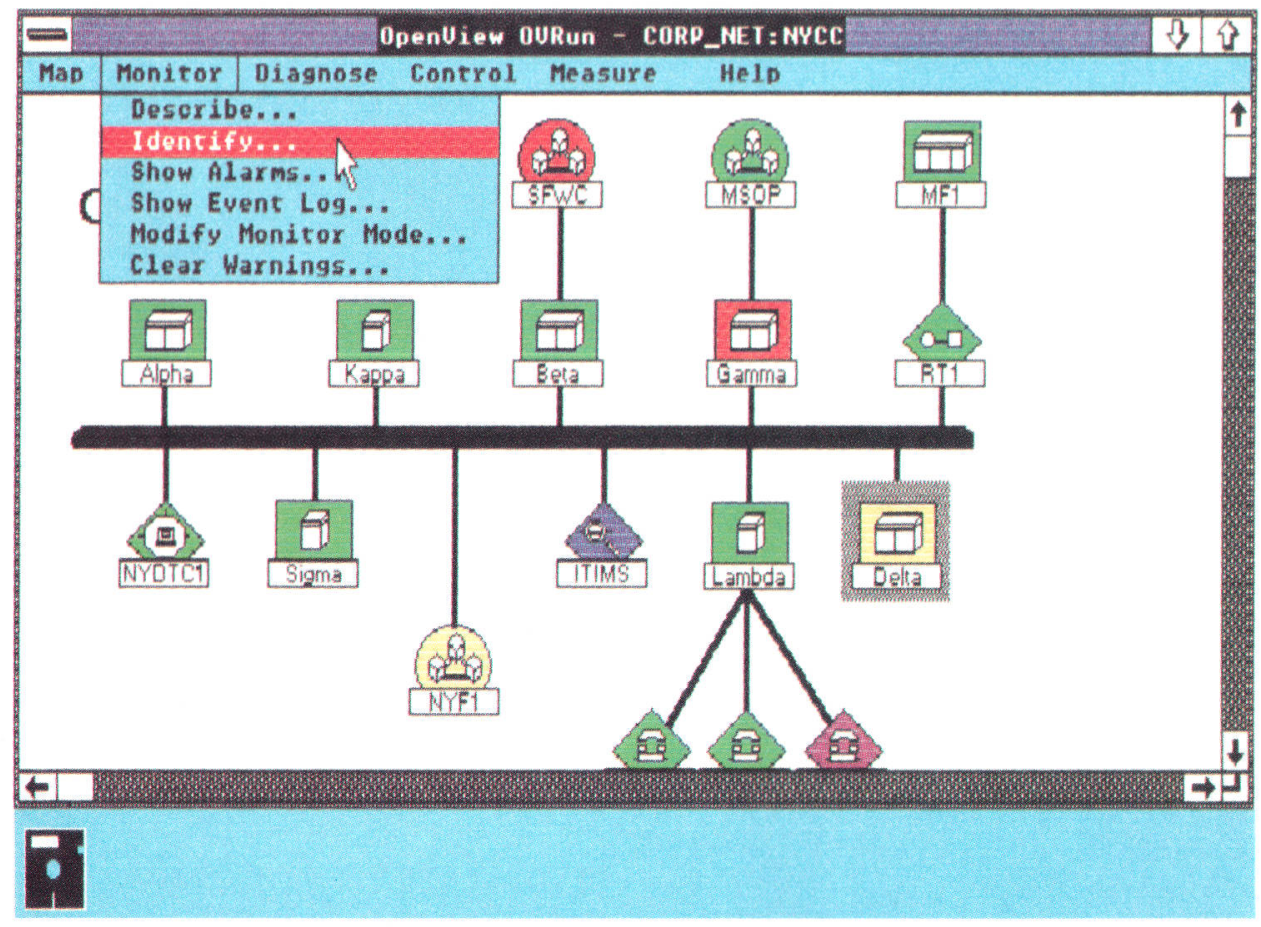

(a)

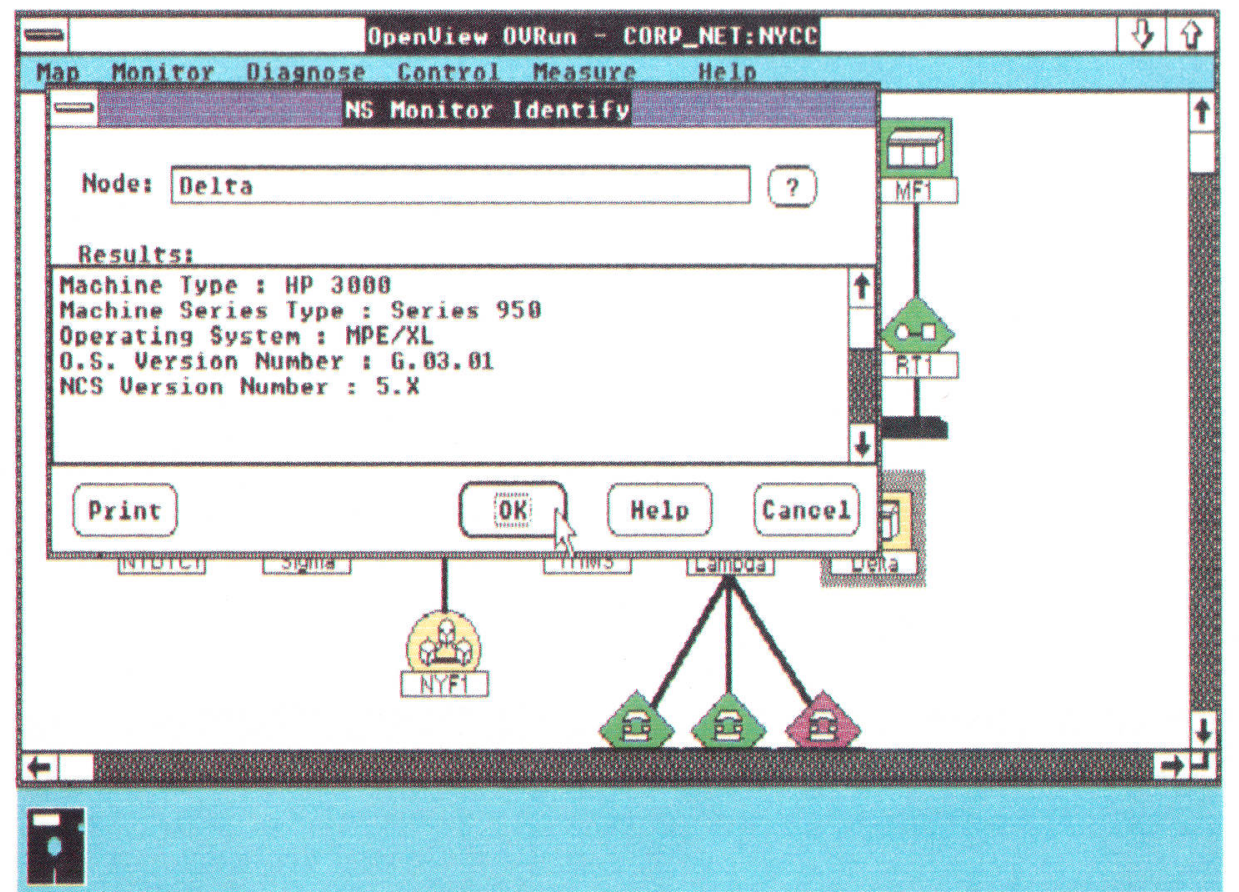

(b)
Fig. 2. (a) The user selects the computer symbol labeled Delta and the Identify menu item. (b) This symbol/menu item combination provides the user with some identification information about the computer system Delta. 
The map functionality in HP OpenView Windows allows the user to navigate through the map in many different ways. The user can double click on a subnet symbol to display the network that the subnet symbol represents, or the user can navigate through the map by using the following menu items contained in the map menu.

- Go To Top allows the user to view the top picture of any network currently being viewed.

- Go To Previous allows the user to view the previous picture displayed.

- Go To Picture allows the user to view a picture listed in a dialog box. By clicking on one of the items in the box and then clicking OK on one of the the pictures listed, the selected item is displayed.

- Go To allows the user to find pictures containing the symbol for a selected node or device. A dialog box will be displayed listing all the pictures containing the symbol if the symbol is in more than one picture.

Based on feedback from developers, two features were added to the map handling facility: treating lines as symbols and allowing multiple applications to register for the same symbol.
Lines. In the early versions of HP OpenView Windows, many different line types were defined, but they couldn't be managed like other network symbols. We discovered that many network management applications wanted to be able to manage lines. This was especially true of HP's telecommunications divisions. Where the systems divisions thought in terms of boxes, the telecommunications divisions thought in terms of lines.

Since we wanted the user interface to be useful to all network management applications, lines were made equal with other network component symbols. Lines can change color to reflect the status of the line. They can be named, registered for, selected, and managed. Lines can also be labeled and split into different internal types. For example, obj_line can be split into line types obj_line1, obj_line2, and so on. This feature allows multiple applications that register for lines to coexist together. Without this feature, two applications that want to manage lines and use some of the same menu items would not be able to run in the same HP OpenView Windows together. The article on page 71 provides an example of the use of HP OpenView Windows line types for network management.
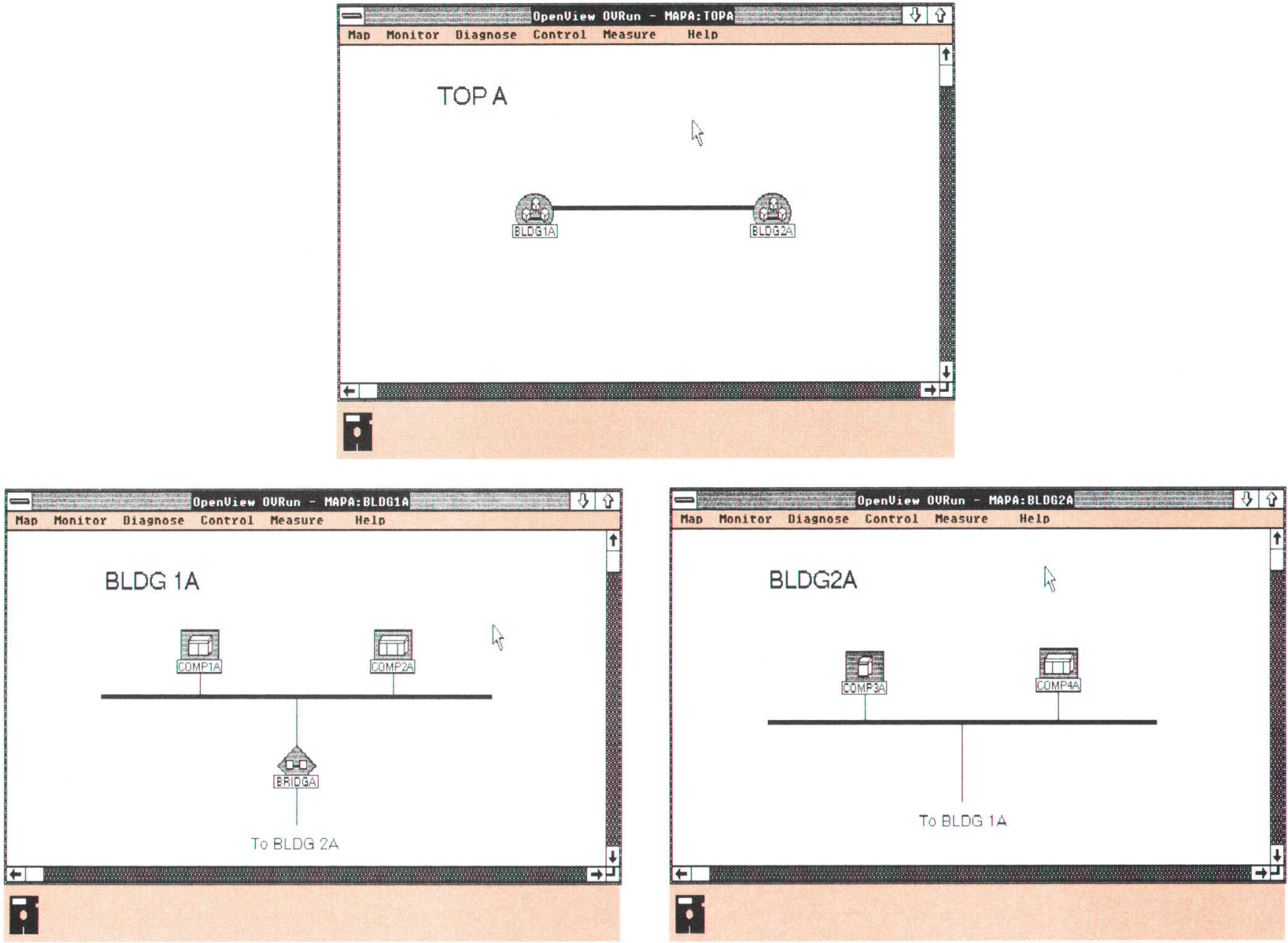

Fig. 3. Hierarchical network map. Note that the subnet symbol is used to depict the existence of network configurations in different buildings. 
Multiple Symbol Registration. Since more than one application can run at a time, more than one application may want to register for the same symbol. This is especially true for applications that manage system symbols like HP DeskManager, SNA, and TCP/IP. Therefore, we allow multiple applications to register for (and to manage) the same symbol with the limitation that they cannot register for the same menu items. This means that if application A is registered for the menu item/symbol combination Identify/DTC, application B cannot cannot register for this same combination, but it can register for another menu item/DTC combination. This limitation ensures that HP OpenView Windows knows to which application to send the selection message. Allowing multiple applications to register for one symbol also works well for integrating foreign applications that need very different functionality. For example, an application that manages a computer system can run together with a terminal emulator connected to that system. When the user selects the computer system, the menu items for both the system management application and the terminal emulator can be enabled.

\section{Menu Integration}

Customers buy network management solutions to gain increased use of network resources and lower the cost of maintaining networks. Lower maintenance costs come through minimizing the time required to train operators and managers. Making the user interface as easy to use as possible is one way of lowering the training costs. Another way of lowering training costs is through the use of a consistent user interface across all network management applications. This means that if a user is tracking down a network problem, there is no need to switch back and forth between different tools with different user interfaces to accomplish the job. There are a wide variety of devices in a network that need to be diagnosed or configured, and if a user has to learn many different user interfaces for each device and type of activity performed, training becomes a significant cost to the customer.

To help ensure a consistent user interface, HP OpenView Windows provides three types of menu items: standard HP OpenView Windows menu items, application dependent menu items, and application-added menu items. Standard
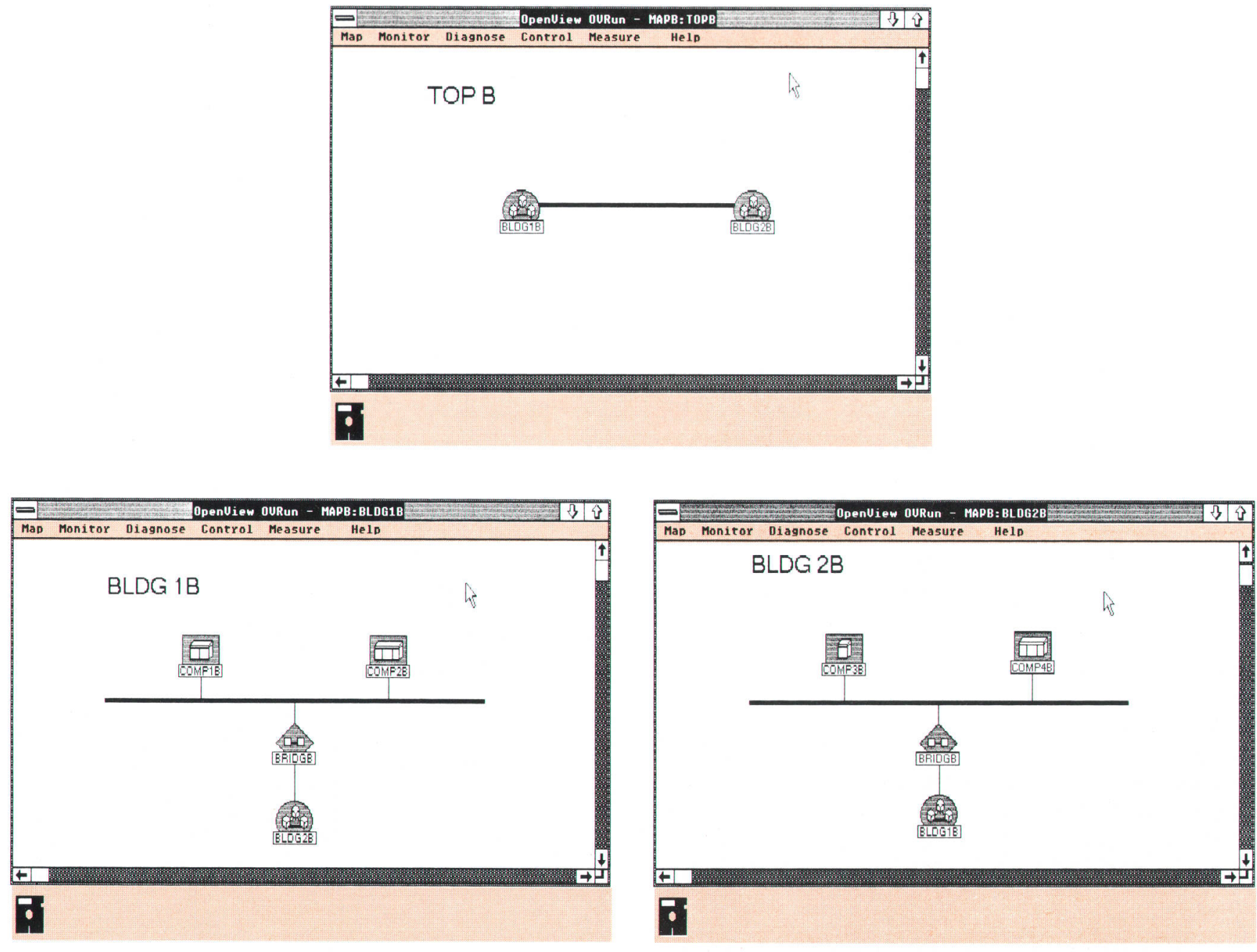

Fig. 4. Network model map. In this representation the subnet symbol for the buildings is shown in each picture. 
HP OpenView Windows menu items appear regardless of what applications are being used. The functionality of standard HP OpenView Windows menu items is always supplied by HP OpenView Windows, not by the application. The OVRun map menu items such as Go To Top and Go To Previous are examples of standard menu items.

Application dependent menu items are displayed by HP OpenView Windows but rely on applications to provide functionality. They appear on the menu regardless of what applications have been installed. The Indentify menu item described earlier is an example of an application dependent menu item. As shown in Fig. 2 the functionality for Identify in this example is provided by the application NS Monitor.

Application-added menu items are added by an application, and appear only when that application has been installed.

Menu items may be object-specific or nonobject-specific. Object-specific items remain disabled (greyed out) until the user selects a symbol in the map. If there is an application that provides the functionality for that type of symbol, it is enabled. An example of an object-specific menu item is the Identify menu item, which is enabled only if an object to be identified has been selected.

Nonobject-specific items are always enabled and can only be handled by one application. An example of a nonobject-specific menu item is the Show Alarms menu item. It is always enabled since the alarm list doesn't refer to any specific object.

\section{Status}

A graphical user interface allows the network operator to gain a large amount of information about a network from the components on the display. The use of color helps the operator absorb this information quickly. Color representing the state of network elements is a key part of HP OpenView Windows.

When HP OpenView Windows is initialized the status of all of the devices is unknown. This state is represented by the color blue. Once the applications start coming up and informing HP OpenView Windows of the states of the devices, the colors are changed to represent the true state of the devices. Red is used for critical, yellow for warning, green for $\mathrm{OK} /$ normal, and magenta for an informational state. The informational state might be used to indicate that the device is off-line or under test, or has messages queued that don't represent a warning condition. If a device changes to a critical or warning state, in addition to changing the color of the symbol, a warning message is displayed. Since lines are treated the same as symbols, their colors represent the same state information. The only difference is that the initial color for lines is black.

Since HP OpenView Windows allows the user to have many different pictures representing different parts of a network, it is possible that the user may not be viewing the picture that contains a device whose status has just changed because the device may be at another level of the network hierarchy or on another network. One of the features of the HP OpenView Windows map is status propagation. This means if a symbol in a picture changes state (color), all subnet symbols representing that picture will have their color changed to represent the highest severity contained in the picture. In the map examples shown in
Figs. 3 and 4, if COMP1A had a critical error, the symbol COMP1A in the BLDG1A picture would be red. The subnet symbol BLDG1A in picture TOPA would also turn red, indicating to the user that at least one symbol in picture BLDG1A had a critical severity. Because the user may have multiple levels of subnets, status information must be propagated up through multiple levels. If the user had drawn the network in a network model map like the one in Fig. 4, this would mean that every subnet would be red. If COMP1B were critical (red), BLDG1B would be red because it contains COMP1B, and BLDG2B would be red because it contains subnet symbol BLDG1B. In HP OpenView Windows, the user can configure the map to propagate status up one level or all levels.

\section{Help Facility}

The last area where HP OpenView Windows provides integration of applications is in the area of context sensitive help. The NewWave help facility ${ }^{1}$ is used to allow applications to format and integrate help text. The user is able to access the help facility by either pulling down the Help menu or by clicking on the Help button within a dialog box. The Help pull-down menu has menu items for HP OpenView Windows and for each HP OpenView Windows application installed. Selecting a menu item under the Help menu brings up the index for HP OpenView Windows or the HP OpenView Windows application. Clicking on the Help button within a dialog box brings up the help screen for that dialog box.

\section{Conclusion}

HP OpenView Windows is a tool for both network management application developers and end users. For developers, HP OpenView Windows simplifies the task of developing a graphical user interface for network management applications by providing functionality in the areas of map handling, menu integration, status, and help. End users also benefit from HP OpenView Windows, since the resulting applications have a consistent user interface that is easy to learn and use.

\section{References}

1. V. Spilman and E.J. Wong, "The HP NewWave Environment Help Facility," Hewlett-Packard Journal, Vol. 40, no. 4, August 1989, pp. 43-47. 


\title{
HP OpenView BridgeManager: Network Management for HP LAN Bridges
}

\author{
Since LAN bridges receive all the data packets transmitted \\ on the LAN segments they interconnect, they are an ideal \\ focal point for monitoring packet integrity and the number \\ of packets forwarded and filtered.
}

by Andrew S. Fraley and Tamra I. Perez

S LOCAL AREA NETWORKS (LANs) grow to the limits of their physical and electrical specifications, traffic levels can reach a point where throughput is significantly impaired. A LAN bridge logically separates segments of a very large LAN so that optimum throughput is maintained. A bridge allows intersegment communication only as required. For example, if two nodes on the same LAN segment are communicating, there is no need for their packets to be forwarded to other LAN segments (see Fig. 1). Thus, a bridge restricts traffic to only the necessary LAN segments.

To separate LAN segments logically, a bridge monitors traffic on the network and builds an internal address table. In essence, the bridge learns the MAC addresses and port locations of nodes communicating on the LAN, and only forwards traffic to other LAN segments if source and destination nodes are on different bridge ports.

Recently, an IEEE 802.1 committee defined a standard spanning tree algorithm ${ }^{1}$ that allows redundant bridging to increase LAN reliability (see Fig. 2). A spanning tree algorithm is typically used to determine the shortest path between any two LAN nodes. The spanning tree standard adds the capability for two bridges to interconnect the same LAN segments and protect LAN operation in the event of a bridge failure. The spanning tree standard places one bridge in active bridging mode for forwarding packets and the other bridge in backup state for monitoring traffic and

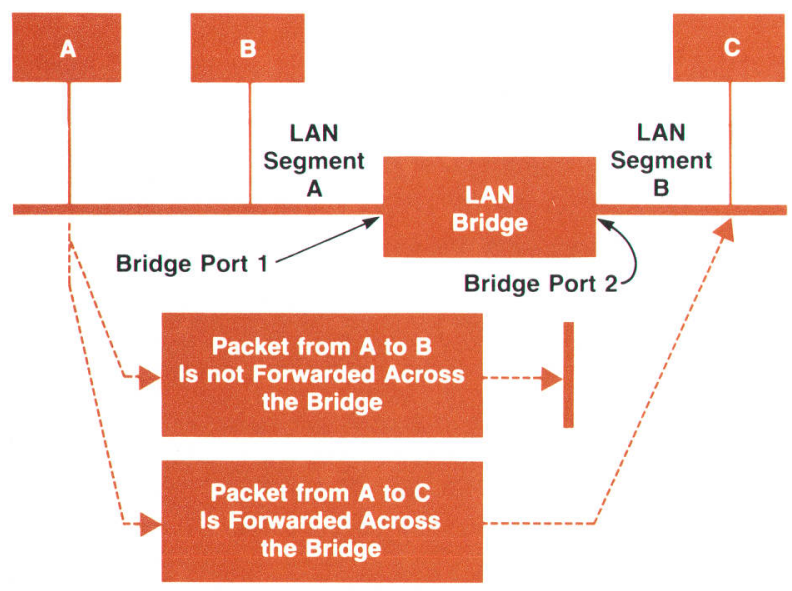

Fig. 1. How a $L A N$ bridge isolates traffic. maintaining its address table. It is important to note that connecting two bridges that do not support the spanning tree standard could result in an infinite cycle of packets between redundant bridges. This would create an increase in the traffic levels on adjacent LAN segments, bringing down both segments.

Hewlett-Packard builds a number of two-port LAN bridges. These bridges interconnect IEEE 802.3 10-Mbit/s networks, IEEE 802.3 1-Mbit/s networks, and IEEE 802.5 token ring networks. The HP products and the networks they support are given in Table I. The 10-to-10 and 10-to-1 bridges that bridge IEEE 802.3 10-Mbit/s and 1-Mbit/s networks support the spanning tree algorithm and network management.

\begin{tabular}{|c|c|c|}
\hline & $\begin{array}{c}\text { Table I } \\
\text { HP LAN Bridges }\end{array}$ & \\
\hline $\begin{array}{l}\text { HP LAN } \\
\text { Bridge }\end{array}$ & Network Connection & $\begin{array}{r}\text { Network } \\
\text { Management }\end{array}$ \\
\hline $\begin{array}{l}\text { HP 28647B Star- } \\
\text { LAN Bridge }\end{array}$ & $\begin{array}{l}802.310-\mathrm{Mbit} / \mathrm{s} \text { to } 802.3 \\
\text { 1-Mbit/s }\end{array}$ & Yes \\
\hline $\begin{array}{l}\text { HP 28648B LAN } \\
\text { Bridge }\end{array}$ & $\begin{array}{l}802.310-\mathrm{Mbit} / \mathrm{s} \text { to } 802.3 \\
10 \mathrm{Mbit} / \mathrm{s}\end{array}$ & Yes \\
\hline $\begin{array}{l}\text { HP 28649A Token } \\
\text { Ring Bridge }\end{array}$ & $\begin{array}{l}802.310-\mathrm{Mbit} / \mathrm{s} \text { to } 802.5 \\
\text { 4-Mbit/s }\end{array}$ & No \\
\hline
\end{tabular}

\section{Why Manage a Bridge?}

There are two reasons to manage a bridge: to monitor the network and to configure the bridge. Because bridges receive all the data packets on the segments they connect, they are an ideal focal point for monitoring packet integrity and the number of packets forwarded and filtered. This

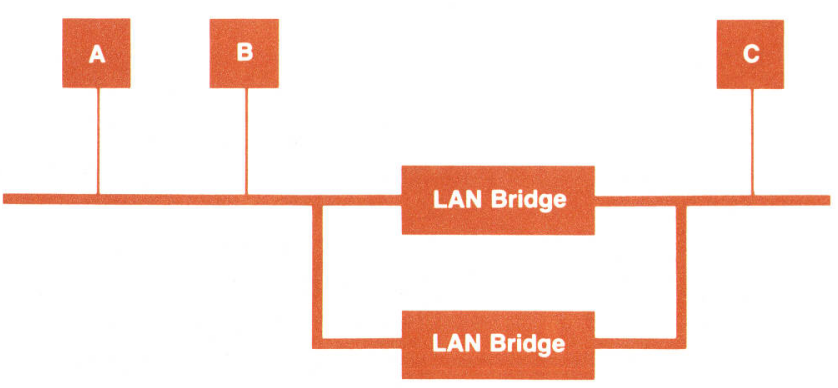

Fig. 2. Redundant bridge configuration. 
information can be used to tune the network and isolate problems. For example, if a bridge port sees sustained traffic levels over twenty-five percent, this indicates that the segment may have too many nodes. Any subset in which the nodes generate a lot of traffic communicating among themselves should be broken into a separate segments and isolated by a bridge. For another example, if a new node was added yesterday, and today the bridge port to which it is attached is reporting a high level of CRC errors, this indicates a problem with the transmitter on the new node.

Bridge management can also be used to customize the configuration of the bridge's operating parameters. For example, if there is a set of nodes containing sensitive data that should be accessed only by a handful of privileged users, these sensitive nodes can be placed on a private segment and isolated by a bridge. The bridge's address table can be configured to forward only packets from the sensitive nodes and from the handful of privileged nodes. Consider a LAN that has grown so large that the worst-case forwarding time between two bridges exceeds a second. In this case, it might be necessary to adjust the spanning tree algorithm time-outs upward for optimal spanning tree performance.

\section{HP OpenView BridgeManager}

The HP OpenView BridgeManager is an HP Vectra computer-based HP OpenView application that manages HP's 10-to-10 and 10-to-1 LAN bridges. The BridgeManager provides the ability to poll bridges, read parameters, set parameters, upload and download complete configurations, log on and log off, log counters, and monitor alarms. The BridgeManager also supports the HP NewWave help system, which has been integrated into the HP OpenView product.

The BridgeManager is divided into two parts: the user interface and the network interface. The user interface interacts with the HP OpenView system and Microsoft Windows. The network interface manages the communication with the LAN bridges.

\section{User Interface}

The BridgeManager user interface provides a graphical interface based on a network map consistent with other HP OpenView applications. About half of the BridgeManager user interface code provides links to Microsoft Windows, HP OpenView Windows, and the BridgeManager network interface (see Fig. 3). The other half of the user interface code implements a table-driven formatter that formats packets received from a bridge and parses user input into packets sent to a bridge.

The parsing and formatting functions were implemented in a central formatting mechanism for two main reasons: to reduce code size and to ensure flexibility. In Microsoft Windows, segments can be discarded or swapped from system memory and new segments loaded from disk. When Microsoft Windows runs low on system memory, this swapping or discarding begins to occur and performance degrades. Therefore, it is imperative to keep the size and number of code segments small. The formatting mechanism meets the code size goal by trading off code size for data size. Instead of hard-coding formatting statements for each

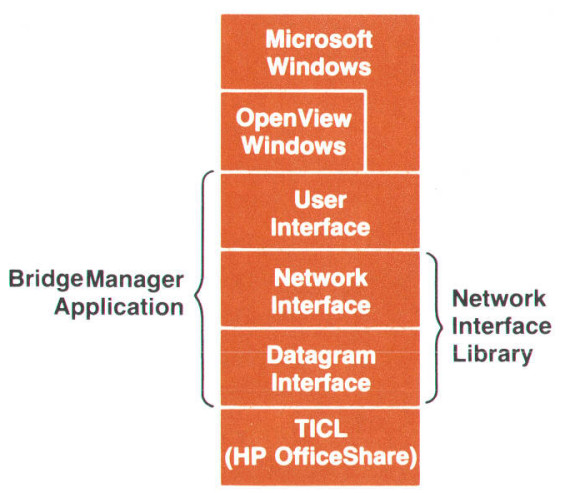

Fig. 3. Layers of software involved in the HP OpenView BridgeManager application.

packet type, the formatting directives are described in a data table and a shared formatter interprets the table.

The network management protocol used by the BridgeManager is loosely based on an HP proprietary network management protocol that was targeted for use in HP's network management products until standard network protocols became available.

Throughout the project, the BridgeManager protocol changed to add or delete functionality. The BridgeManager user interface was designed to minimize the time required to adapt to a changing protocol. The formatter achieves this flexibility goal because if the packet format is changed, no code is affected. Only the table must be modified to describe how to format and parse the modified packet.

The formatting table is an array of field descriptors. A field descriptor contains information describing the field type (word, string, MAC address, etc.), the default, minimum, and maximum values for the field, the offset of the field in the result string, a pointer to the location of the field in the incoming packet buffer, and a flag that is TRUE if the field is the last field in the string being created (each string may have multiple fields). The following fragment of a real formatting table describes the formatting of the two lines:

$\begin{array}{ll}\text { IEEE802 Link Address } & \text { 080009-0033C4 } \\ \text { Product Number } & \text { HP 28647B }\end{array}$

contained in the list box of the BridgeManager Identify dialog box shown in Fig. 4. 
struct

int field_type;

char *min_max_default;

int field_offset;

char ${ }^{*}$ packet_location;

int last_field;

\}

counters $=\{$

FORM_RESSTRING,$\quad \mu^{*}$ String from string table

NULL, $\quad /{ }^{\star}$ Nomin/max information

$0, \quad \quad I^{*}$ Field at beginning of line

(char*) STRING_IEEE_802_LINK_ADDRESS, /*ID of */

/*string table string */

FALSE,

${ }^{\star}$ Not the last string in line

*/

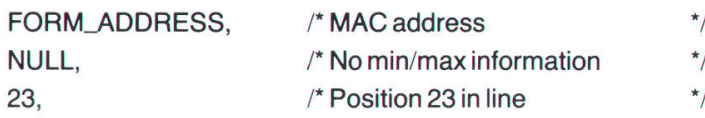

(char *)packet.ieee_802_source_addr, /* Data position */

/*in packet */

TRUE,

${ }^{*}$ Last field in line

FORM_RESSTRING,

NULL,

0 ,

$/{ }^{\star}$ String from string table

/*No min/max information

${ }^{*}$ Field at beginning of line

(char *)STRING_PRODUCT_NUMBER, / I ID of string */

$/$ *table string

FALSE,

${ }^{*}$ Not last field in line

FORM_STRING,

"20,"

23 ,

${ }^{\star}$ String from packet

${ }^{*}$ Max length of string is 20

${ }^{\star}$ Position 23 in line

(char *)packet.product_number, $\left.\right|^{*}$ Data position in packet */ TRUE ${ }^{*}$ Last field in line

\}

Consider how this table is used to interpret an identify packet. The first field is of type FORM_RESSTRING. Knowing the field is a resource string, the formatter interprets the packet_location pointer not as a pointer into a packet, but as a constant identifying which string is to be loaded from a Microsoft Windows resource string table. This string is loaded at offset 0 in the result string. Because the lastfield flag is FALSE, the formatter realizes that the resulting string is not yet complete. The next field is of type FORM_ADDRESS. The formatter interprets the packet location pointer as a pointer to a MAC address and formats the packet data into the string at offset 23 in the result string. Because the lastfield flag is TRUE, trailing spaces in the result string are trimmed and the string is output by the formatter to a list box or file (as directed by the code that invoked the formatter). The next field is another field of type FORM_RESSTRING. It is handled exactly like the first resource string field. The last field is of type FORM_STRING. The formatter treats the packet location pointer as a pointer to a string. This is the only field in the example whose min_max_default string pointer is not NULL. The "20" is scanned from the string and the formatter ensures that the string in the incoming packet is 20 characters or less. If longer than 20 characters, the string is truncated. The lastfield flag is TRUE so the second result string is trimmed and sent to a list box or file. The two result strings produce the two lines shown above.

When the user wants to change parameters on a bridge, the formatter also takes strings that have been modified through interaction with the user and parses them into outbound packets. The rest of the user interface deals with the network interface to send and receive packets, to interact with Microsoft Windows objects such as list boxes, edit fields, and pushbuttons, and to interface to the network map and the HP OpenView C-tree data base.

To illustrate how the BridgeManager user interface interacts with these three entities to implement a function, consider the Identify function. When the user wishes to read identity information from a bridge, the user selects the bridge from which to read in the network map and then selects the Identify menu item in the Monitor menu. When the HP OpenView system detects that a bridge is selected, it sends a message to the BridgeManager's message queue indicating that the Identify menu item was activated. The BridgeManager responds to this message by calling Microsoft Windows to create and display the Identify dialog box.

Each BridgeManager dialog box has an associated dialog box function that handles messages for that type of dialog box. When the Identify dialog box is displayed, Microsoft Windows sends an initialization message to the dialog box function. When the Identify dialog box function receives the initialization message, it loads the selected bridge's text label and MAC address into the window's Bridge Label and Bridge Address fields shown in Fig. 4. To do this, the BridgeManager uses HP OpenView function calls to get an object identifier specifying which bridge was selected. The BridgeManager then uses this identifier to look up the bridge's label and MAC address by calling an HP OpenView function. The dialog box function then retrieves an identify packet from the selected bridge using the network interface. When the packet is returned, the formatter formats the information into the list box for the user to view.

\section{Network Interface}

The most important function of the BridgeManager's network interface is to manage the communications exchange between bridges and the management node. A management node is the system from which a network manager uses the BridgeManager application to monitor a network. A large part of this functionality is devoted to the maintenance of incoming packets.

Before beginning a discussion of internal packet manage- 
ment, it is helpful to understand the architecture and general operation of the BridgeManager network communication process. As shown in Fig. 3, the BridgeManager network interface resides in several layers of code. HP OfficeShare is HP's network transport software. The transport interface compatibility layer (TICL) in the HP OfficeShare software provides access to the network hardware via interrupts. The datagram interface running on top of the HP OfficeShare software limits access to TICL as a protection mechanism. To send a packet across the network, the BridgeManager network interface makes a call to the datagram interface, which filters down to a hardware transmission request. When the network hardware receives a packet, it interrupts TICL. TICL then interrupts the upper layers of software until the packet reaches the destination application, which in this case is the BridgeManager.

Recall from the discussion of the user interface that the Microsoft Windows memory manager dynamically swaps code and data segments. Interrupts from the network hardware would fail if the network interface code and data segments were allowed to move dynamically in memory. Therefore, the network interface is implemented as a static Microsoft Windows library that is separate from the user interface. The user interface must poll the network interface to receive any packets that may have arrived asynchronously. When the network interface receives a poll request and has a packet available for the user interface, it copies the packet from its buffer structure into the pointer provided by the user interface. The network interface then frees the packet buffer to accept another packet.

Two types of packets are received at the BridgeManager network interface: a response to a previously posted request packet (hereafter known as a response packet) or an event notification packet. Event notification packets arrive asynchronously. They are sent when the bridge detects a change in the network state that might be of concern to the user. Event notification and response packets coexist in the network interface buffer structure. Therefore, an array is used to maintain four maximum-size IEEE 802.3 packets. Two of the array elements are used for response packets and the remainder for event notifications. The following is a code fragment containing the packet buffer declaration.

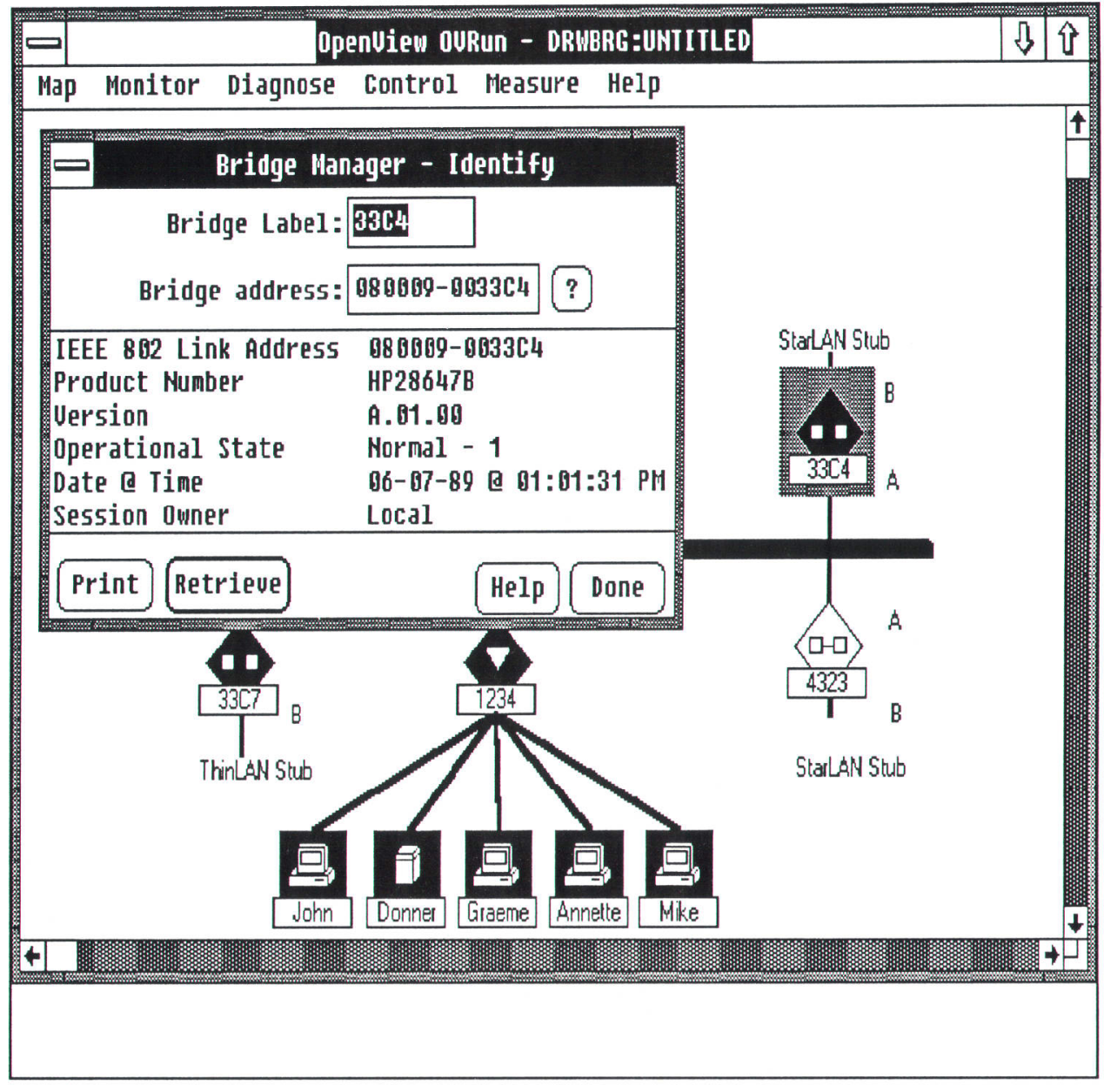

Fig. 4. BridgeManager Identify dialog box. 
struct \{

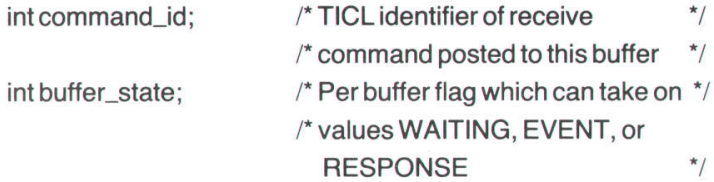

/* Receive packet buffer

$l^{\star}$ Allocate four receive buffer

$/ *$ structures (allowing applications */

$/{ }^{\star}$ to buffer up to four back-to-back */

${ }^{\star}$ packets)

The network interface uses the buffer_state and command_id elements to control the packet buffer structure. The command_id element is returned from TICL and is used in the event that the receive request must be canceled. The buffer state element is used to determine whether a buffer is waiting to receive a packet and also to maintain the balance of buffer use between event notification and response packets.

It is critical that the incoming packet buffers not be filled with only response packets or only event notification packets. If the network interface packet buffers were filled with response packets, event notification packets would be locked out of the management node, thus crippling the management feedback system. On the other hand, if the packet buffers were filled with event notification packets, the user would not receive responses to management commands issued on the network. To avoid this problem, the interrupt service routine responsible for validating and accepting incoming packets is designed to monitor the state of the packet buffer with every packet arrival. Currently, two packets of either response or event notification type are allowed to coexist in the buffer structure. If two packets of the same type exist in the buffer and a third arrives, it will be thrown out to maintain the buffer balance.

A major design goal of the BridgeManager was that it coexist with other management nodes and be a well-behaved application with respect to CPU and memory use. The network interface library resides in static nonswappable memory. Therefore, a major design goal was to keep the library as small as possible. The code size was not a problem, but the data structures, specifically the packet buffer, were our major concern. During development, we determined that two buffers per packet type were more than sufficient to ensure that the network interface did not become a a performance bottleneck. However, if this condition changes in the future, the number of buffers for each type of packet is easily altered. A recompilation is required to implement the change.

\section{Conclusion}

Bridges are important network components that segment local area networks into logical subnets, filter traffic between subnets and the network segments, and add reliability to the network through redundant paths. Since a bridge observes network traffic during operation, adding management to bridges provides valuable network information to the user.

\section{Acknowledgments}

Special thanks to project manager Phill Magnuson, teammates Ralph Bean and John Reilly, and the bridge hardware team.

\section{References}

1. Local Area Network Standard - MAC Bridges, ANSI/IEEE Standard P802.1D, Rev. 6, September 1988. 


\title{
HP OpenView Data Line Monitor
}

\author{
Monitoring large and complex network configurations is \\ crucial to maintaining the integrity and performance of data \\ communication lines. The HP OpenView Data Line Monitor \\ is a hardware and software solution for monitoring these \\ data communication lines.
}

\author{
by Michael S. Hurst
}

NFORMATION NETWORKS are becoming larger and more complex and efficient management of these networks is crucial as organizations become more dependent on them. At the heart of any network are the physical data communication links that connect the computers together. For wide area networks these links often consist of point-to-point leased analog lines and it is problems with these lines that are the most common cause of trouble in data communications. Network managers and datacom managers in places such as corporate data centers are therefore increasingly concerned with the performance and integrity of their data communication lines.

Point-to-point leased lines connect data equipment in separate locations. Normally four-wire lines with two circuit pairs are used, one line for each direction of transmission. Modems interface the data equipment to these lines. Imperfections that cause data errors on analog lines fall roughly into two groups: steady-state impairments (e.g., noise or amplitude modulation) and transient impairments (e.g., impulses or signal dropouts). In addition, telephone companies may condition lines to meet the attenuation distortion (frequency response) and delay distortion (envelope or group delay) characteristics required by modern high-speed modems.

The HP OpenView Data Line Monitor (OVDLM) is an analog leased-line monitoring system for multivendor networks, based on the HP 4948A in-service transmission impairment measuring set (ITIMS). ${ }^{1}$ The HP 4948 A permits the testing of lines while they are still in use. Conventional testing of analog lines requires the lines to be taken out of service while test signals, such as test tones, are applied. Alternatively, modem-based line monitoring and management systems are available from manufacturers of datacom equipment. However, these systems are usually proprietary and may require specialized and expensive smart modems. The HP 4948A works with ordinary modems in the range of 2.4 to $14.4 \mathrm{kbits} / \mathrm{s}$ that are compatible with AT\&T or CCITT standards. HP OpenView Data Line Monitor is compatible with the other HP OpenView network management applications and can run concurrently with them.

The HP OpenView Data Line Monitor software controls a single HP 4948A and one or more HP $3777 \mathrm{~A}$ channel selector access switches to monitor or troubleshoot analog datacom circuits at one location. Each HP 3777A switch can access up to 30 four-wire circuits and can be cascaded to achieve the required access capacity to a maximum of 31 switches. The functionality of OVDLM is provided en- tirely by the software running in the HP OpenView workstation. Unlike some of the other HP OpenView applications, OVDLM is not split into two parts, with one part running on a remote computer. Instead, the ITIMS and switches are controlled directly from the HP OpenView workstation via the HP-IB (IEEE 488, IEC 625). The HP-IB has the advantage that its high speed enables the ITIMS and up to 13 access switches to be controlled from just one interface card in the HP Vectra PC. (Because of the electrical limit of 15 devices on one HP-IB, two interface cards are required for up to 27 switches, and three for the maximum of 31 switches). In many installations it is possible that the HP OpenView workstation might be situated at some distance from the communications rack where the ITIMS and switches are located. The distance limit of 20 meters with HP-IB cable can be overcome by using HP-IB extenders. A pair of HP 37204A multipoint HP-IB extenders using fiber optic cable allows HP-IB extension up to 1250 meters with negligible loss in performance. For greater distances, such as when the HP OpenView workstation and the ITIMS are at different sites, a pair of HP 37201A HP-IB extenders can extend the HP-IB to unlimited distances over ordinary tele-

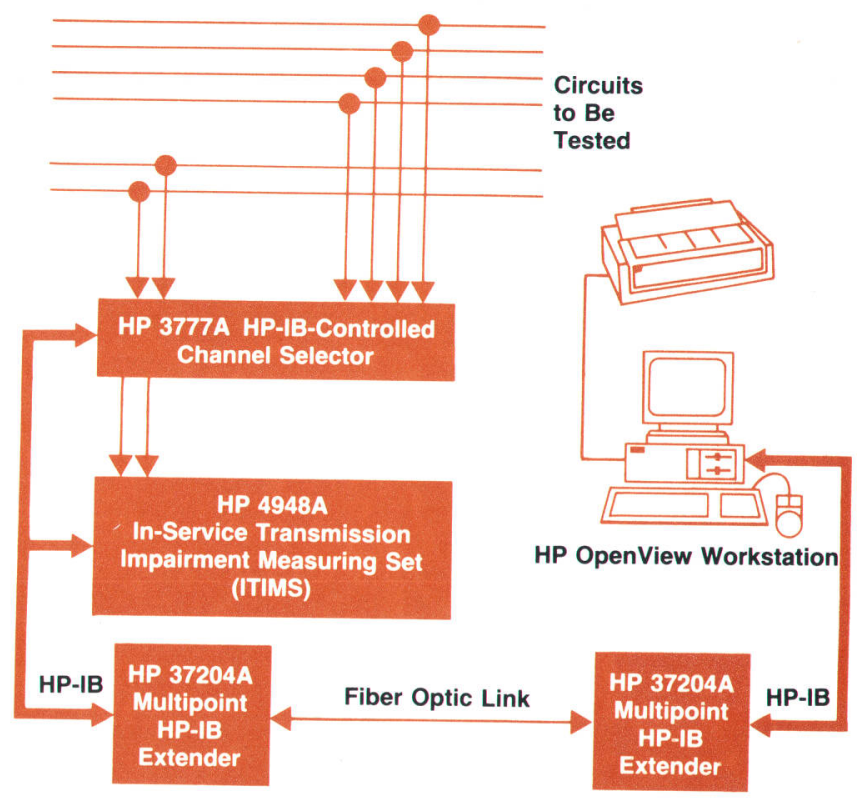

Fig. 1. A typical equipment configuration for using the HP OpenView Data Line Monitor (only one access switch, the HP 3777 A channel selector, is shown). 


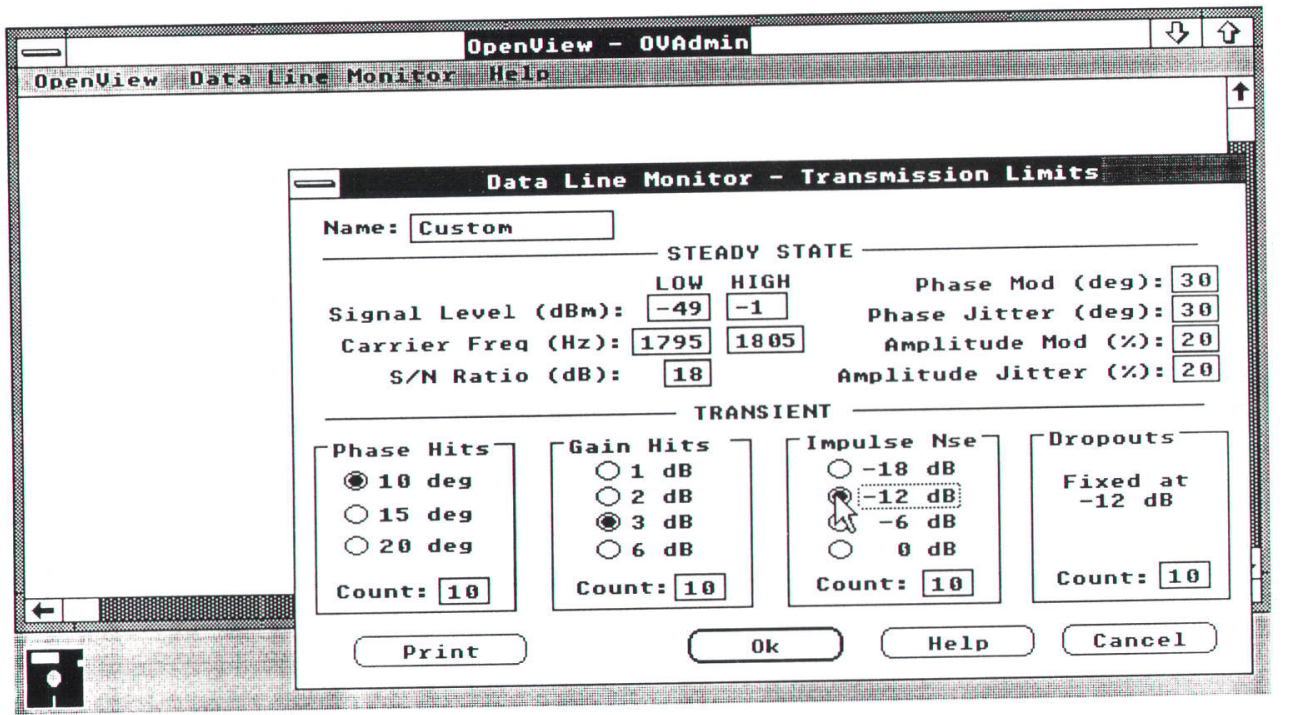

Fig. 2. Screen used to enter transmission limits. phone lines, albeit with a loss in performance. Fig. 1 shows a typical equipment configuration with one access switch.

For routine alarm monitoring, OVDLM can be set to test all lines automatically in sequence. For troubleshooting, a single line can be selected and monitored continuously. This is particularly powerful for trapping intermittent faults. A description of all lines to be monitored is stored in the HP OpenView Windows data base, including details of the modem type and transmission performance limits. When OVDLM detects a problem with a particular line, the color of that line is changed to red and a message is displayed in the HP OpenView alarm window. OVDLM can report line performance in two ways. First, routine day-to-day monitoring typically uses an alarm-only mode, which indicates when any of the key analog parameters for each line go outside their predefined limits. Secondly, when data is required for line performance benchmarks or trend analysis, OVDLM is able to store the maximum, minimum, and average values of all line characteristics during a selected measurement period. The results for each line are stored in a common log file which can be viewed at any time.

Like all HP OpenView applications, OVDLM is divided into three programs: OVAdmin, OVDraw, and OVRun. The article on page 60 describes these programs.

\section{OVDLM and OVAdmin}

In OVAdmin the user (a network or datacom manager) can define sets of transmission and conditioning limits. Transmission limits include specifications such as the minimum and maximum signal level, minimum signal-to-noise ratio, maximum number of dropouts allowed in a 15-minute interval, and so on. Conditioning limits define attenuation distortion and delay distortion masks. Typically these sets of limit values are based on AT\&T or CCITT specifications for analog leased lines. Each set of limits is given a name, which is then used to specify the limits for a particular line. Many lines can thus share common sets of limits. The sets of limits are stored in a special OVDLM data file rather than the HP OpenView Windows data base. Where the data is stored is not apparent to the user. Fig. 2 shows the screen used to enter the transmission limits and Fig. 3 shows the

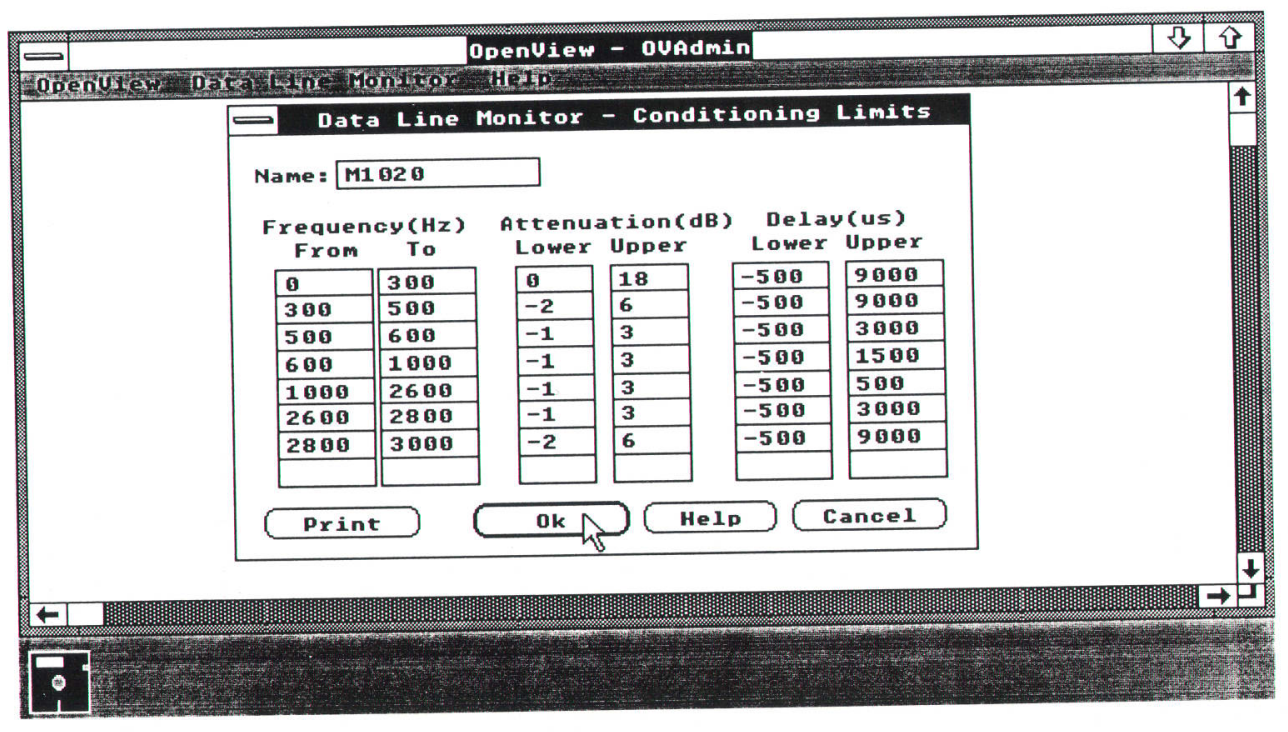

Fig. 3. Screen used to enter conditioning limits. 


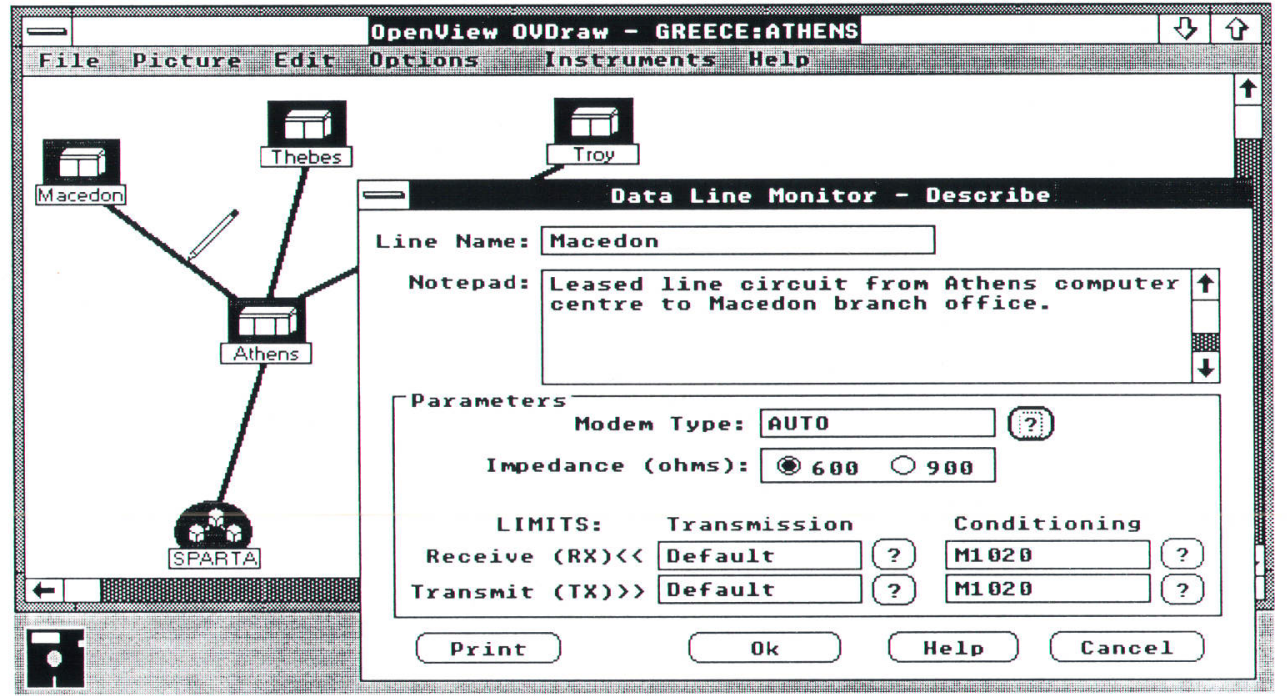

Fig. 4. The Describe dialog box is used to describe datacom line characteristics. The pencil is used to select the desired line. screen used to enter the conditioning limits. The data shown entered in Fig. 3 is the attenuation and group delay mask from CCITT recommendation M.1020 for international leased analog circuits.

\section{OVDLM and OVDraw}

In HP OpenView Windows, lines drawn on the network map can be managed in the same way as computer or component icons. OVDLM registers with HP OpenView Windows to manage these lines. In OVDraw the user draws the datacom lines on the network map, describes their details, and enters configuration information about the ITIMS and switches. Lines are described in exactly the same way as computer or component icons are described in other HP OpenView applications, that is, the user selects the Describe menu item from the Edit menu, moves the pencil cursor over the line and clicks the mouse button. This brings up the line Describe dialog box. OVDLM extends the default line description of HP OpenView Windows to include details of the line impedance, the modem type, and the names of the line transmission and conditioning limits (as set up in OVAdmin). Fig. 4 shows a sample network map with a line Describe dialog box. Note that the modem type is set to AUTO. When this line is monitored in OVRun, the ITIMS will automatically search for the correct modem type by examining the live line signal.

A difference between lines and most other managed objects is that lines are passive because they need separate tools (the instruments) to do the management. The ITIMS and switches therefore have to be described in OVDraw. To accomplish this an Instruments menu was added to the OVDraw menu bar containing the Data Line Monitor menu item. Selecting this menu item will bring up an ITIMS Instruments dialog box on which the HP-IB interface select code and the address of the ITIMS and first-level switch can be entered (see Fig. 5). From this dialog box a Connect Line dialog box can be accessed for connecting lines to the ports of the first-level switch. A Connect Switch dialog box can also be selected for connecting a second level of switches to the first-level switch. Subsequently lines can be connected to the second-level switches.

\section{OVDLM and OVRun}

The OVRun part of OVDLM controls the program's

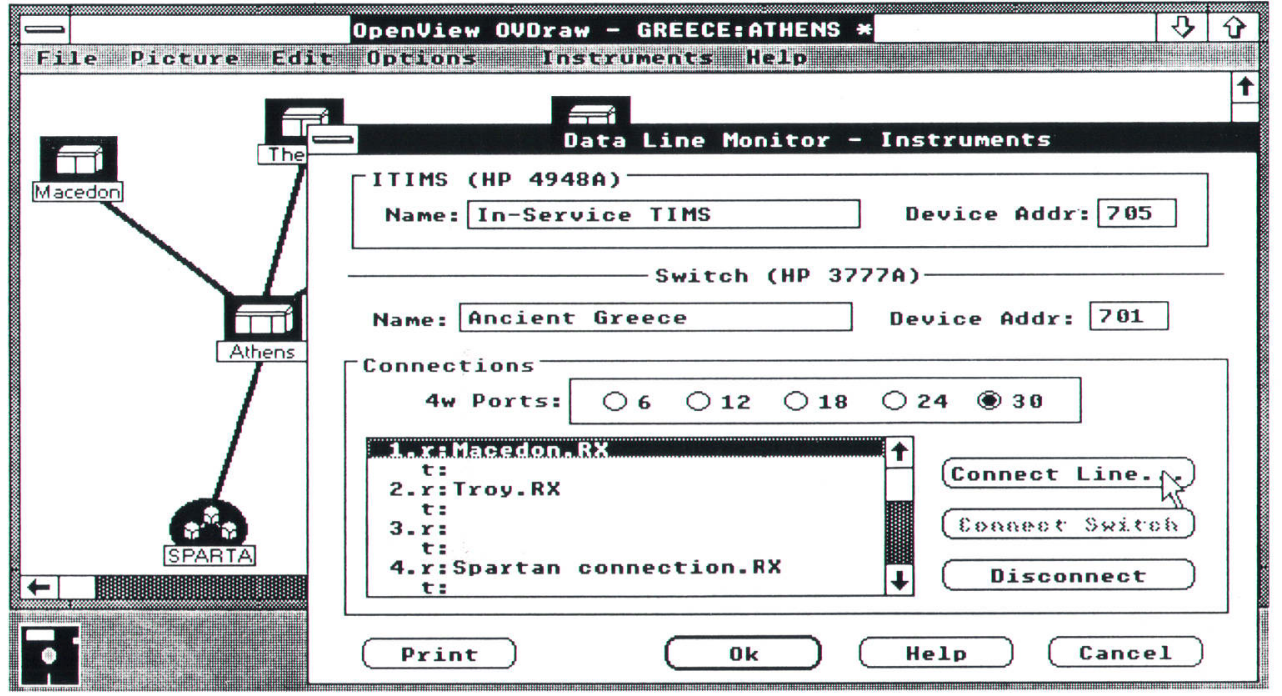

Fig. 5. Instruments dialog box used to describe the HP-IB interface codes and the various connections between the line and switch boxes. 


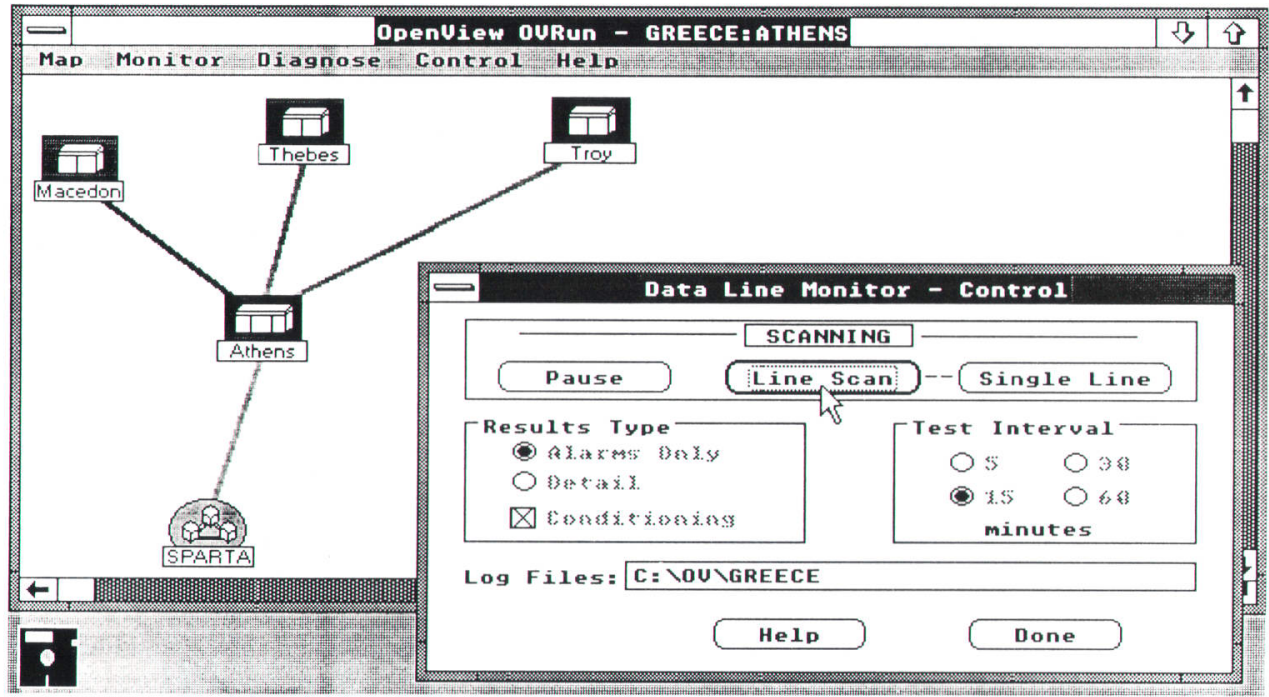

Fig. 6. Control dialog box with Line Scan menu item selected, Alarms Only results, and 15-minute test intervals. monitoring activities. The user sets up global monitoring parameters and starts and stops the monitoring via a Control dialog box accessed through a data line monitor menu item on the Control menu. The Set Parameters menu, which is a default menu item supplied by HP OpenView Windows, is not used for this function because it requires a network line name to be selected first. Entering a line name was found not to be intuitive for setting the global monitoring state because the global status information is not associated with any particular network line.

The Control dialog box allows the monitoring state to be set to line scan mode or single-line mode, along with the required results and a test interval. The name of a disk file to hold the results can also be entered. Fig. 6 shows a Control dialog box with the Line Scan menu item selected, Alarms Only results, and a 15-minute test interval selected. The Results Type and Test Interval controls are greyed (dimmed) because they can only be altered when monitoring is paused.

In line scan mode OVDLM will monitor each line in turn for a duration set by the test interval $(5,15,30$ or 60 min- utes). For example, if there are 30 4-wire circuits and a five-minute test interval is selected, a complete scan of all the circuits will take about six hours (assuming both the transmit and receive circuit pairs of each line are to be tested and allowing a minute per test for ITIMS training). If alarms-only results are selected, a summary of the impairment violations is written to the results log file at the end of the test interval (C:IOVGREECE in Fig. 6). If conditioning is selected as well as alarms only, the attenuation and delay results will be checked against the conditioning limits (see Fig. 3) and a pass/fail result will be written to the log file. If detail results are selected, the maximum, minimum, and average values of the steady-state line impairments, along with the number of transient events, are recorded at the end of the test interval. If conditioning and detail results are selected together, the complete attenuation and delay data is recorded as well.

In single-line mode OVDLM will continuously test only one line that has been selected on the network map. The Results Type selection works similarly to line scan mode, except that in Alarms Only, each individual violation is re-

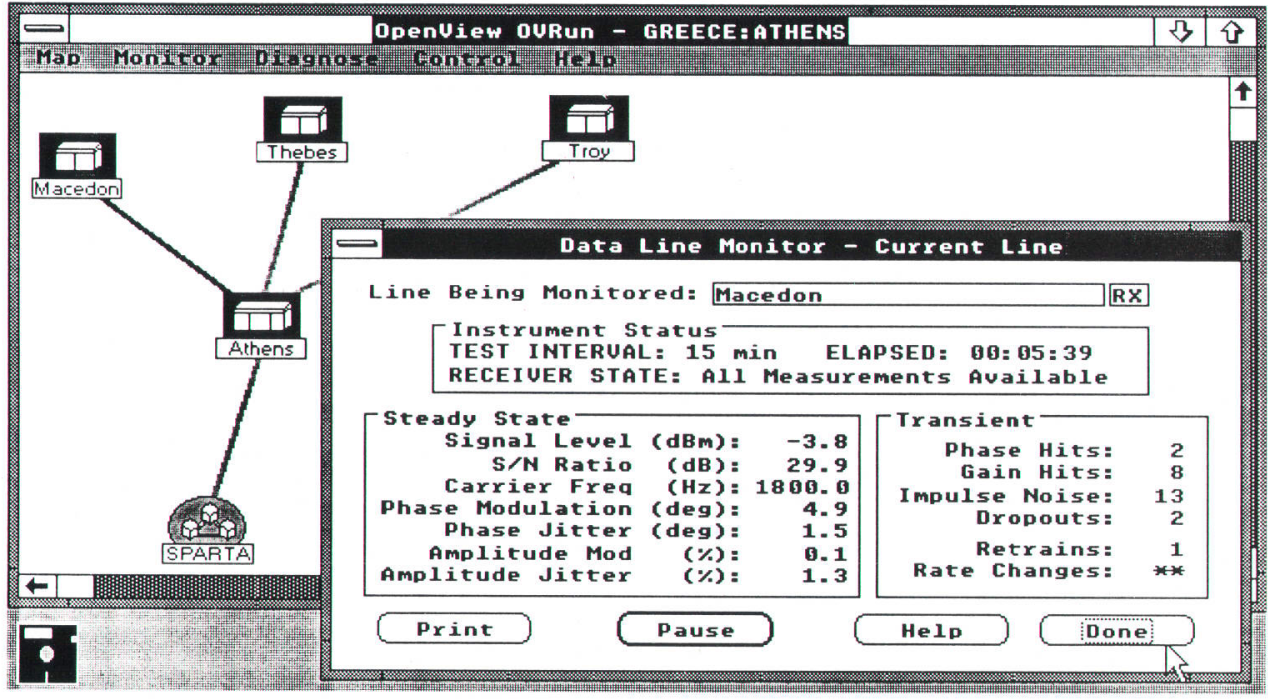

Fig. 7. An example of a current line window. 


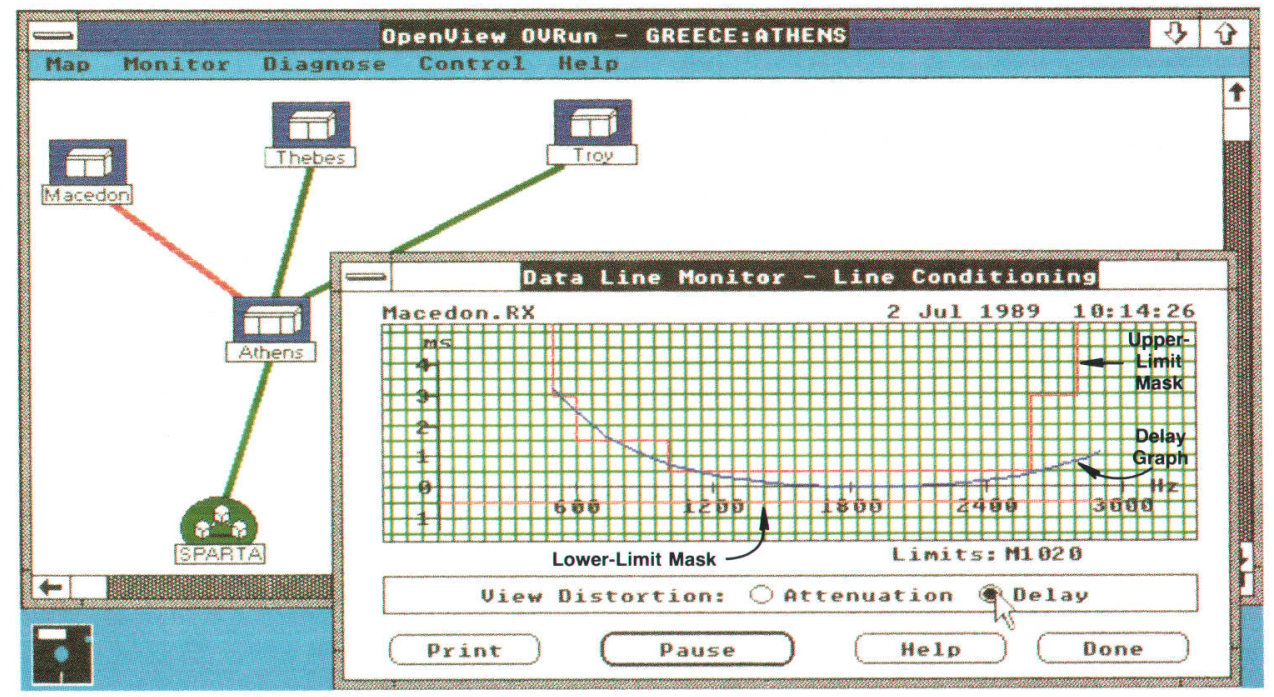

Fig. 8. An example of a line conditioning window. The data for the upper-limit mask comes from the delay upper column in Fig. 3 and the lower-limit mask comes from the delay lower column in Fig. 3 corded in the log file. This is useful for tracking down intermittents on a particular circuit.

Once started, monitoring runs in the background even if all the OVDLM dialog boxes have been closed and the user is working in another HP OpenView Windows application. Thus the user is not normally aware of the monitoring process. However, during HP-IB input/output, the Vectra can appear busy for a few seconds, especially when reading back the line conditioning data from the ITIMS. This is because asynchronous HP-IB input/output is not supported in the Microsoft Windows environment. To inform the user when HP-IB input/output is in progress, OVDLM changes the cursor icon to an HP-IB busy icon consisting of an hourglass and an HP-IB logo. At the end of the test interval the color of the line on the network map is changed to green if the measured performance is within limits and to red if it is outside one or more limits. In the latter case an alarm message is sent to the HP OpenView Windows alarm window.

During monitoring the user can choose to view either real-time results for the line currently being tested or historical results for a line taken from the log file. These results are accessed through Current Line and Line History menu items on the Diagnose menu.

Selecting the Current Line menu item will display a current line window and a line conditioning window. The current line window shows the steady-state and transient results and is refreshed from the ITIMS every twenty seconds. Any results that are outside the limits for the line are shown in red. The conditioning window displays a graph of attenuation versus frequency or delay versus frequency, using the ITIMS data-spectrum results and the conditioning mask limits for the line. This window is only refreshed every minute, the period with which the ITIMS remeasures the data spectrum. Fig. 7 shows an example of a current line window. Here the impulse noise and retrains counts are above limit and hence shown in red. As a result, the line on the map is also turned red. Fig. 8 shows a sample line conditioning window. Note that the delay graph moves outside of the mask below about $1000 \mathrm{~Hz}$.

The Line History menu item allows browsing through the
OVDLM log files for historical results of a particular line. Filters are available to select the types of results required. For example, there are filters that can be used to narrow the selection to alarms results or details results for a particular transmit or receive pair.

\section{Using Log Data}

It is important for datacom managers to track the longterm performance of their lines. The OVDLM log files are in ordinary ASCII text and organized so that they can be easily read by other application programs. For example, the data can be imported into a spreadsheet program, such as Microsoft Excel, and trend analysis done on the detail results for each line. This trend analysis allows lines with deteriorating performance to be spotted before they become critical. Demonstration Excel macros to do this are supplied with the OVDLM software.

\section{Acknowledgments}

Acknowledgments are due John Duff, who designed and coded the OVDLM software, and Mark Dykes, Garry Irvine, and Elaine Butterwith for advice on many aspects of the definition and implementation of OVDLM.

\section{Reference}

1. N. Carder, et al., "In-Service Transmission Impairment Testing of Voice-Frequency Data Circuits," Hewlett-Packard Journal, Vol. 38, no. 10, October 1987.

Microsoft is a U.S. registered trademark of Microsoft Corp. 


\title{
Network Management for the HP $\mathbf{3 0 0 0}$ Datacom and Terminal Controller
}

\author{
The HP OpenView DTC Manager software is responsible \\ for controlling, monitoring, and diagnosing the DTCS on a \\ local area network. Its functions can be exercised either \\ from a local workstation on the LAN or from an HP Response \\ Center or other remote workstation.
}

\section{by Serge Y. Amar and Michele A. Prieur}

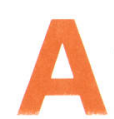

NEW GENERATION OF HP 3000 COMPUTERS was born in 1986. The distinctive features of this generation are HP Precision Architecture, ${ }^{1}$ the MPE XL operating system, ${ }^{2}$ and the input/output structure. ${ }^{3}$

One of the peculiarities of the input/output structure is the way terminals and printers are connected to a host HP 3000 computer. They are connected through a controller (originally called the distributed terminal controller or DTC), which is connected to a local area network. Originally, because the DTC code and configuration file were too big to fit in nonvolatile memory, the host was in charge of downloading them to the DTC at power-up. The host was also responsible for building the DTC configuration file and for managing the DTC (reset, upload, self-test, etc.).

For the first release of the MPE XL software, the LAN was used more as an I/O bus than as a network, in the sense that a terminal plugged into a DTC could establish a connection to one and only one host computer even if the LAN was shared by more than one host. Moreover, some important features were missing, such as wide area network access (X.25).

With the release of the MPE XL 2.0 operating system in October 1989, major new functionalities are implemented in the DTC: X.25 access, PAD (packet assembler/disassembler) support, terminal I/O switched connections, back-toback connections, and others. All of these services can be shared by multiple MPE XL systems connected to the LAN. In keeping with its expanded capabilities, the DTC has been renamed the datacom and terminal controller.

The DTC now offers LAN-accessible shared services, and is no longer tied to one host system. With the back-to-back feature, it can even work without any hosts on the LAN. For these reasons, a new way had to be found to manage the DTC and its services. This is the function of the HP OpenView DTC Manager workstation.

\section{The HP OpenView DTC Manager}

The HP OpenView DTC Manager is the network management software for the new services of the DTC. It is responsible for controlling, monitoring, and diagnosing the DTC.

The HP Openview DTC Manager software runs on an HP Vectra ES/12 personal computer with a VGA display, a 40-megabyte hard disk, an HP LAN access card, and a 2Mbyte expansion board. It runs under the Microsoft Windows environment and the HP OpenView Windows umbrella. It implements a graphical user interface, allowing the user

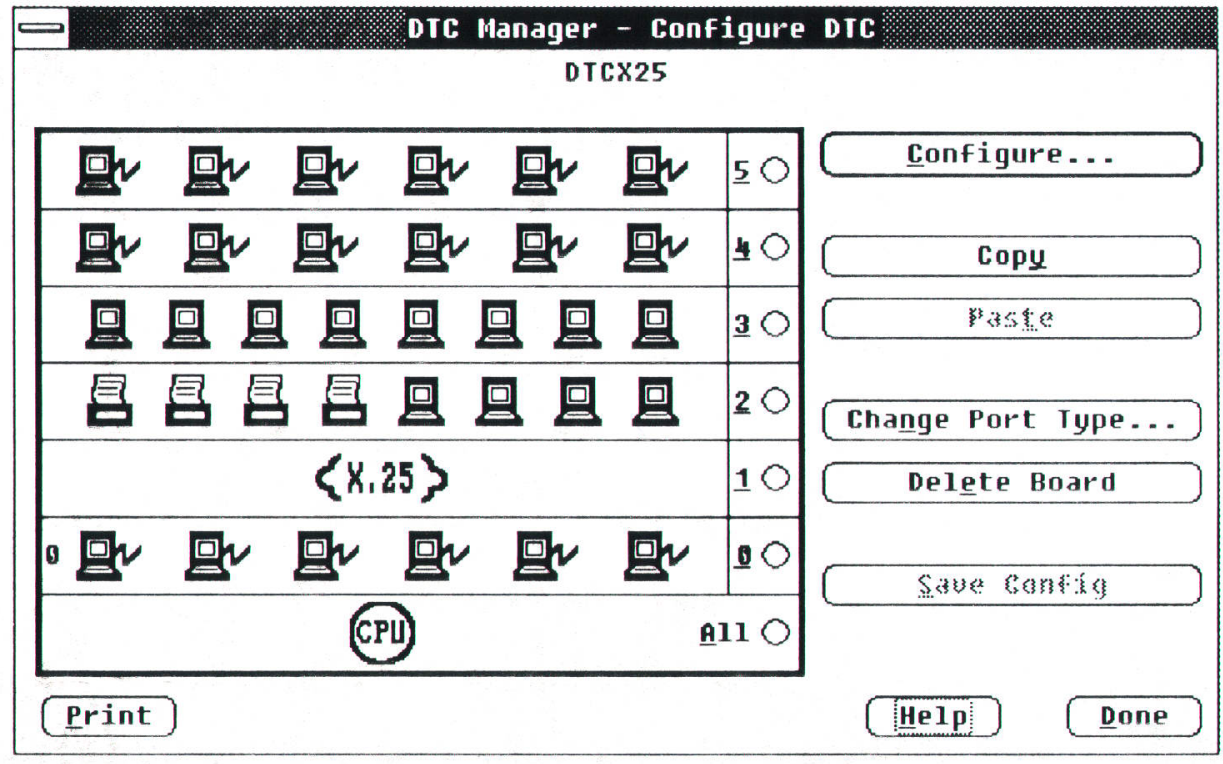

Fig. 1. Most HP OpenView DTC Manager functions begin with the display of the DTC rear panel, which shows the types of cards and devices that are plugged into the DTC. DTC stands for datacom and terminal controller. 
to trigger management functions on the local area network DTC components.

The contributions of the HP OpenView DTC Manager include:

- The use of HP OpenView Windows to allow the user to "touch" the network.

E Easy access to the DTC components through a graphic, logical view of the DTC rear panel.

- Remote access to all of the DTC management functions through a modem connection using the same graphic user interface.

- The ability to add a support tool at any time without modifing the HP OpenView DTC Manager code.

HP OpenView Windows is a network shell that mainly displays and manages graphic maps of networks. At initialization, it spawns the applications that have an entry in the OpenViewApps section of the WIN.INI file (see article, page 60).

It provides services such as map object management, menu and menu items addition, alarms, single-key data base access, and others.

Almost all of the HP OpenView DTC Manager's functions, triggered by the user from the HP OpenView network map, begin with the display of the DTC rear panel, which allows the user to see at a glance the types of cards and devices that are plugged into the DTC (Fig. 1). Using the mouse, the user can easily select the part of the DTC on which to execute the management function. The selectable components are: the CPU board, a card (X.25, direct connect card, modem connect card), a terminal, or a printer. Each of these is represented by a specific icon.

All features of the HP OpenView DTC Manager can be accessed remotely through a 1200-baud or 2400-baud modem. The user interface is completely separate from the input/output functions so that it can be run in a remote PC without the need to pass graphic data through the serial modem link. This structure was chosen to optimize the exchange of information in the case of remote access-only relevant binary data is transferred. A major problem of such a structure is that it is not possible to take advantage of some Microsoft Windows features. For example, a list box can contain more information than can be transferred all at once over the serial link to load the list box. Therefore, the HP OpenView DTC Manager handles the scroll bars of list boxes to navigate through the lists, and only list data to be displayed is transferred.

Hooks have been implemented in the HP OpenView DTC Manager so that a DTC support tool can be added at initialization time, using the DtcManagerApp section in the WIN.INI file. For example, if a dump formatter were implemented following the guidelines, it would be able to take full advantage of the remote access capability of the HP OpenView DTC Manager.

\section{Software Structure}

The HP OpenView DTC Manager consists of four Microsoft Windows components (Fig. 2):

n PCMPRMP. This module is responsible for interfacing with the HP OfficeShare driver and implementing the two management protocols MP and RMP. ${ }^{4,5}$

- DTCMGRIO. This module is responsible for maintaining the data base associated with DTC management and for translating requests coming from the user interface to requests that can be understood by the PCMPRMP module. It is also responsible for deciding whether a download request coming from a DTC must be serviced or rejected.

- DTCMGRUI. This module is responsible for handling the user interface-for example, interpreting requests coming from the user, requesting additional information when needed, and displaying the results of the requests.

n RLS/CA. This module has two parts. RL Switch is responsible for managing the local and remote modes and for switching messages between DTCMGRIO and DTCMGRUI either on the same PC or through a serial modem link. Data is never exchanged directly between DTCMGRUI and DTCMGRIO. CA stands for connect application. It is responsible for the logon and logoff process in both local and remote modes.

Two of these modules, DTCMGRUI and RLS/CA, are HP OpenView Windows applications. An HP OpenView Windows application is, first of all, a Microsoft Windows application, that is, it has a WinMain, which performs initialization and implements the GetMessage/DispatchMessage loop. However, during the initialization process, it must call some HP OpenView intrinsics, and it does not create its own main window. The application's main window is created by HP OpenView Windows and acts as a communication window between HP OpenView Windows and the application. The application must provide the window procedure for this communication window.

Because an HP OpenView Windows application has no

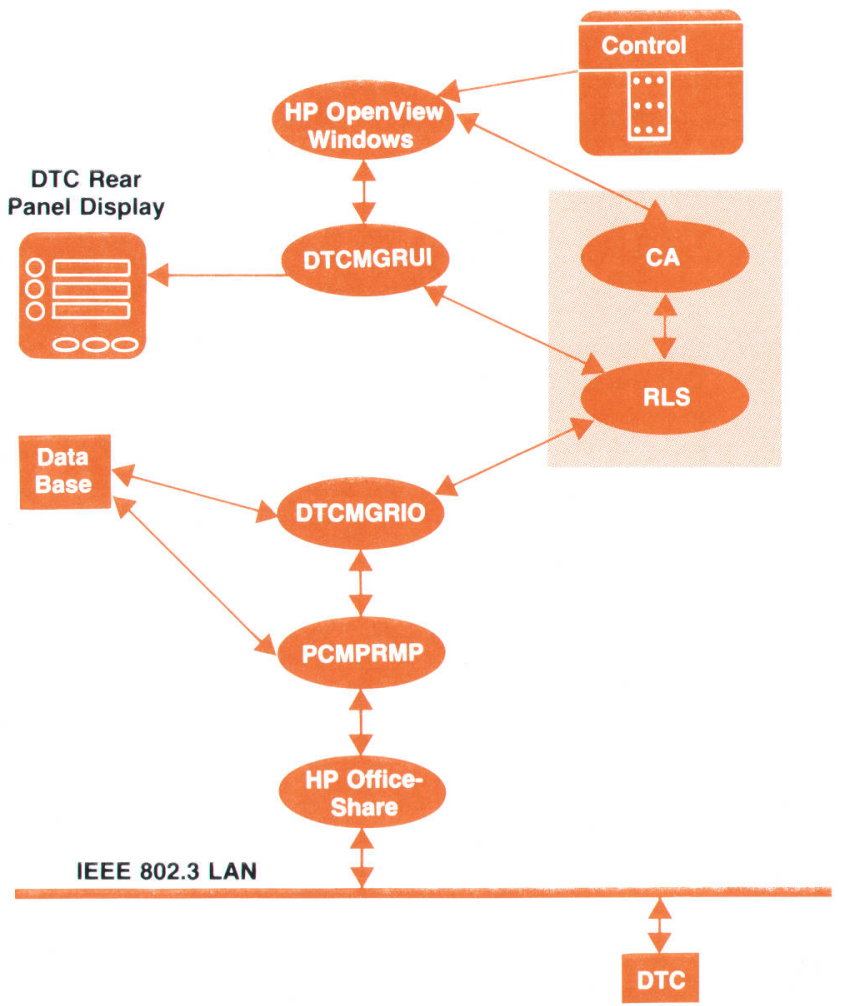

Fig. 2. The HP OpenView DTC Manager consists of four Microsoft Windows components: PCMPRMP (protocol controller), DTCMGRIO (data base manager and translator), DTCMGRUI (user interface), and RLS/CA (communication and logon). 
main window of its own, it has no menu bars. Instead, it requests HP OpenView Windows to add menu items to the main HP OpenView Windows menu bar. An HP OpenView application has to provide for the processing of all the menu items it has registered, and for the processing of some HP OpenView general messages.

\section{User Interface Structure}

The structure of the user interface module (DTCMGRUI) is highly modular (see Fig. 3). It is based on the property of Microsoft Windows that once created, a window is an independent entity able to receive and process its own messages transparently to the process that has created it.

DTCMGRUI consists of a main window and a series of child windows. The main window is the communication window created by HP OpenView Windows during initialization of the application. It is an invisible window and it is mainly dedicated to receiving messages coming from HP OpenView Windows. When the user selects a function managed by the application, HP OpenView Windows sends a message to the communication window.

The window procedure of the communication window is just a dispatcher that creates child windows of the requested class. These are invisible child windows, shown as level 1 windows in Fig. 3. Once it has created the right level 1 child window, the communication window procedure sends it a trigger. Then, it no longer worries about the requested function since it will be processed by the created child window. The communication window simply waits for the Done message sent back by the child window, and then destroys the child window, since this means that the requested function is completed. Every child window is a function of a certain type. For example, there are child windows of type Configure, Set Parameters, Upload DTC, and so on.
Level 1 child windows are only created and destroyed by the main level. Although a child window could destroy itself, for consistency this is never done in DTCMGRUI. The main level is always aware of what is active below it.

In a similar manner, level 1 child windows can create invisible level 2 child windows, which are independent entities for independent subfunctions. Level 1 child windows can also create visible level 2 child windows, which are dialog boxes. Dialog boxes can also be created by invisible level 2 child windows.

One of the main differences between an invisible child window and a dialog box is that an invisible child window is destroyed by its parent, whereas a dialog box destroys itself. Moreover, an invisible child window, when it has completed its function, sends a predefined message to its parent. A dialog box child window, when it is created, receives as a parameter the message it must send back to its parent once it has completed its function. Dialog boxes do not have predefined completion messages.

Each of the child windows, either invisible or visible, is able to communicate with DTCMGRIO through the RL Switch module without the help of the communication window by giving its own handle in any DTCMGRIO request.

\section{Remote Access Structure}

One of the objectives for the HP OpenView DTC Manager was remote access to network management applications. Two PCs with modems running the HP OpenView DTC Manager can communicate and the same DTC management capability is available at both (Fig. 4). For example, from an HP Response Center PC, an HP engineer can manage a customer's DTCs. In fact, only the user interface (DTCMGRUI) and RL Switch run on the Response Center PC. Commands are sent to the DTCMGRIO module of the customer's PC

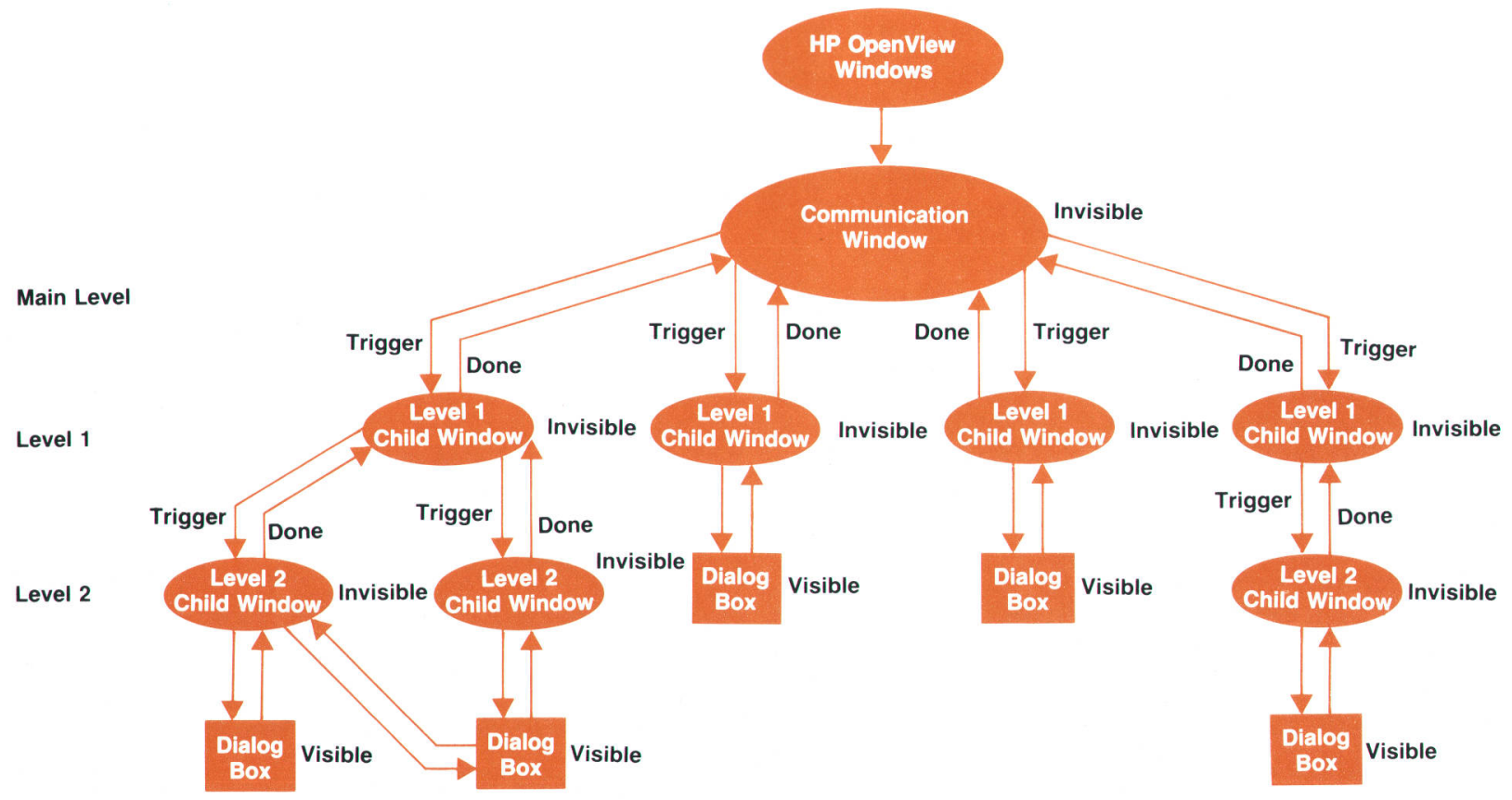

Fig. 3. Structure of the user interface module DTCMGRUI. 
through the serial link.

This functionality is implemented in RL Switch, which is able to switch from local to remote mode and to send orders and replies between DTCMGRUI and DTCMGRIO either locally within the same PC or remotely over the modem line (Fig. 5).

The user is able to log on locally or remotely using the connect application, CA. At initialization, this module adds four menu items to the control menu of HP OpenView Windows: Logon, Logoff, Remote Connect, and Remote Disconnect.

When Logon is activated, Remote Connect and Remote Disconnect become inactive. When Remote Connect is activated, both Logon and Logoff are inactive. Finally, when the user is neither locally logged on nor remotely connected, both Logon and Remote Connect are enabled.

When a user at the Response Center PC chooses the Remote Connect menu item, the DTCMGRUI module in the Response Center PC receives the following sequence of messages:

\section{RL_PREPAREFORCONNECT with param $=$ RESPONSE_CENTER_PC}

\section{RL_LOGONSTATUS}

On the first message, DTCMGRUI just stores the fact that it is no longer in local mode but not yet in remote mode. On the second message, DTCMGRUI stores the fact that remote mode is now active. All HP OpenView DTC Manager menu items are enabled and the inactivity timer is started.

The DTCMGRUI module located in the customer PC receives only the message RL_PREPAREFORCONNECT with param = CUSTOMER_PC. This message is ignored.

Thereafter, except for some very rare cases, whether the HP OpenView DTC Manager is working in remote or local mode is absolutely transparent to DTCMGRUI. There are only two exceptions. First, the display of the Function In Progress dialog box depends on the mode for some requests to DTCMGRIO. Second, in the dialog boxes of type OpenView DTC Manager List, when the items are read one after another and the HP OpenView DTC Manager is in local mode, the items are displayed only when the list box is full to avoid

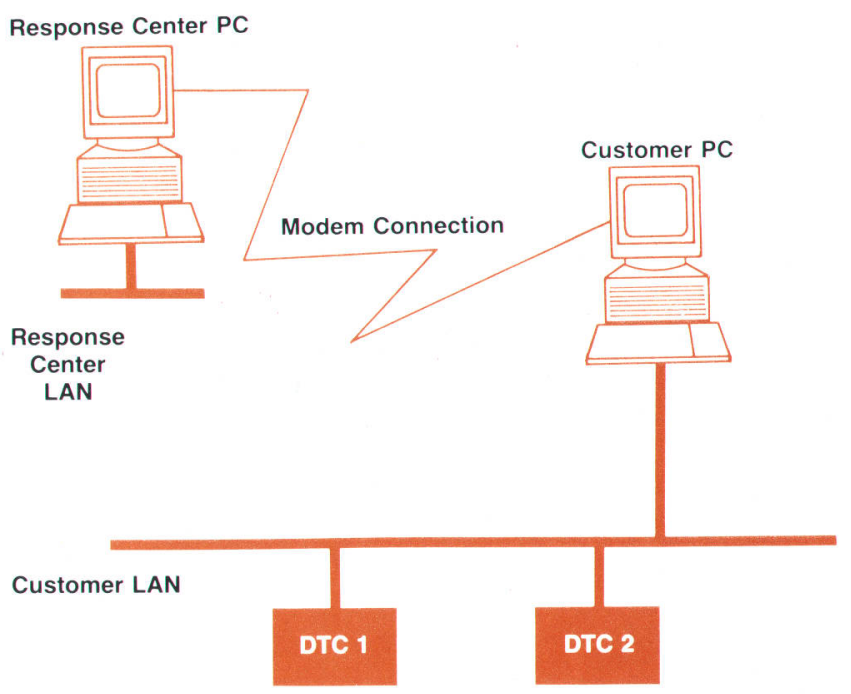

Fig. 4. The HP OpenView DTC manager allows remote access to all DTC functions. For example, from a PC in the HP Response Center an HP support engineer can access a customer's DTCs.

flickering effects. In remote mode they are displayed immediately.

A remote connection is closed if (1) the Response Center engineer completes the job and activates the Remote Disconnect function, (2) the customer requests that the connection be aborted, or (3) the connection between the two modems breaks. In case (1), DTCMGRUI receives RL_LOGOFFREQUEST from RL Switch, and then RL_REMOTECONNECTCLOSED. In cases (2) and (3), DTCMGRUI receives only RL_REMOTECONNECTCLOSED. On RL_LOGOFFREQUEST, DTCMGRUI is free to accept or reject the logoff. If no function is active, the logoff is accepted. If any function is active, the logoff is rejected and the remote disconnect procedure is interrupted. On RL_REMOTECONNECTCLOSED, any active function is aborted and DTCMGRUI assumes that its new status is local and logged off.

Figs. 6, 7, and 8 show the sequence of messages exchanged between CA, RL Switch, and DTCMGRUI in the cases of a remote logon, a remote logoff, and a customer-
Local Mode

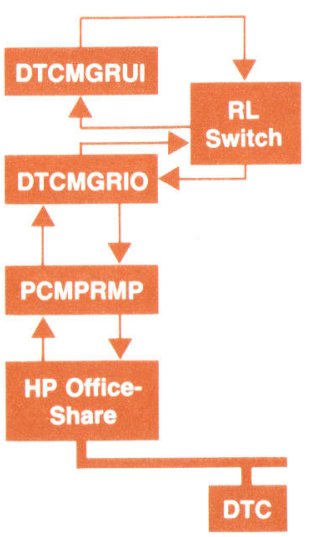

Remote Mode

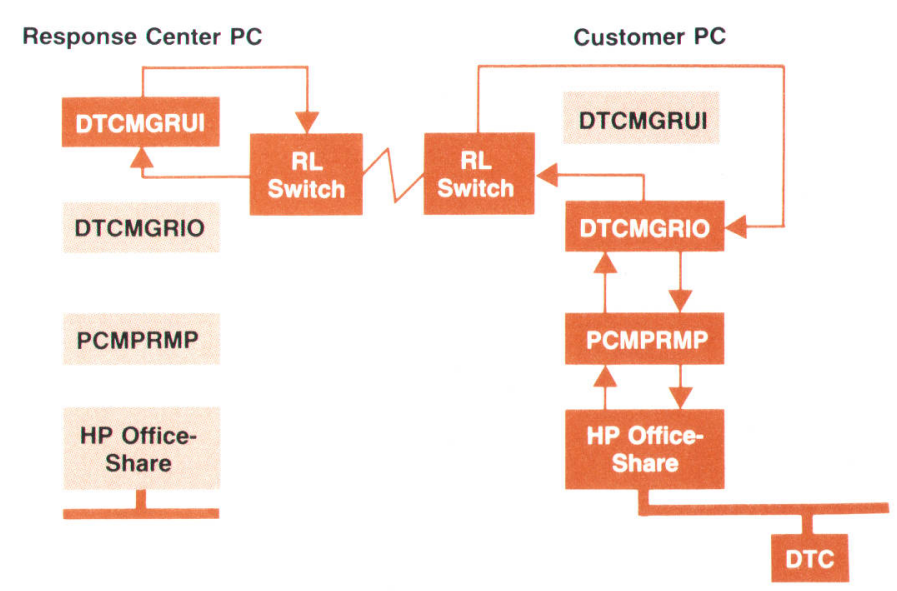

Fig. 5. The RL Switch module has the ability to switch between local and remote modes and route messages accordingly. 


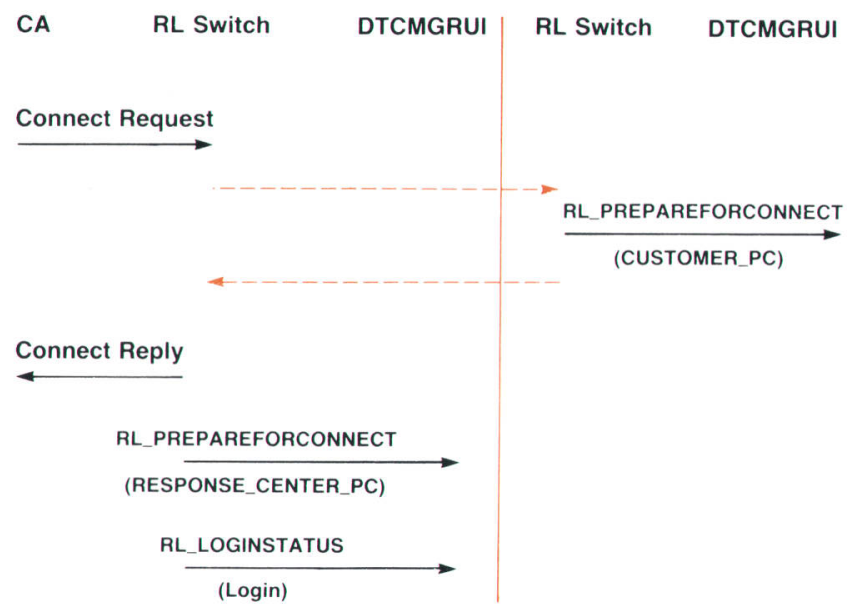

Fig. 6. Messages exchanged for a remote logon

forced abort or a modem line failure.

\section{Dialog Boxes}

Microsoft Windows provides two types of dialog boxes: modal and modeless. Modal dialog boxes do not allow the application (here DTCMGRUI) to do any processing except for handling the dialog box, while modeless dialog boxes allow the application to process both the dialog box and other application windows concurrently.

In compliance with the HP OpenView Application Style Guide, all dialog boxes in DTCMGRUI are modeless dialog boxes. Modeless dialog boxes consume less stack space than modal boxes. They behave as standard child windows and are more bug-free than modal boxes. However, the HP OpenView DTC Manager often needs modal behavior. For example, when a dialog box is displayed, the user is not

Response Center PC

Customer PC

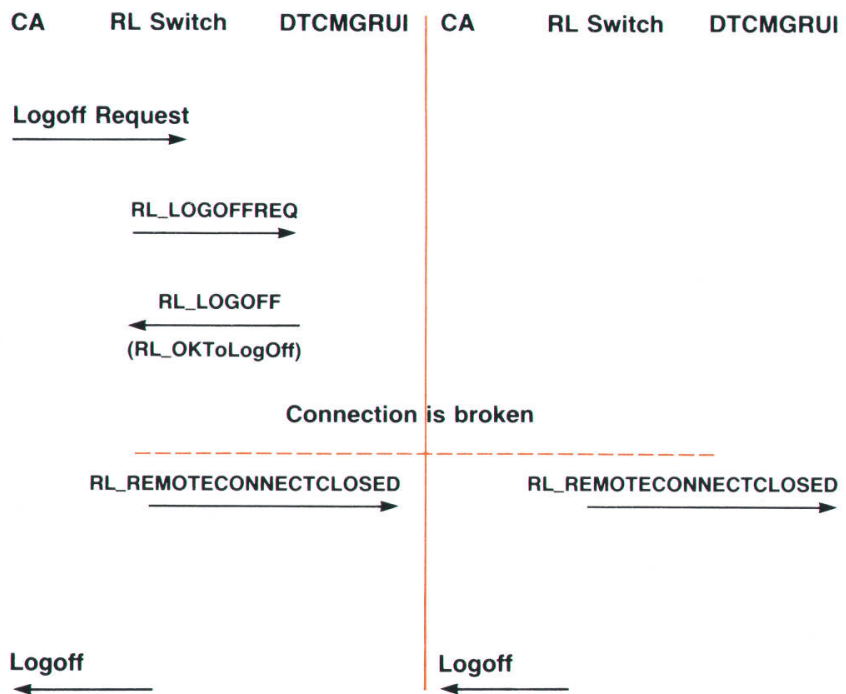

Fig. 7. Messages exchanged for a remote logoff. allowed to do anything except enter information in the box. To meet this need, modal behavior is simulated by creating a modeless dialog box and disabling the window that has created it.

The modeless dialog boxes inside DTCMGRUI can be classified into three categories: classical, HP OpenView DTC Manager lists, and backplane.

The classical dialog boxes are normal Microsoft Windows dialog boxes. They implement list boxes, radio and check buttons, edit fields, and so on. All of these controls are handled by Microsoft Windows after the dialog box is created and initialized. Most of the dialog boxes fall into this category.

HP OpenView DTC Manager lists are dialog boxes that contain list boxes handled by DTCMRGUI instead of Microsoft Windows. These list boxes are initialized with 13 items and are always reset and reinitialized with 13 items maximum. The scroll bar on the right side of the list box is not part of it, but is a scroll bar control handled by the dialog box as an independent scroll bar. The items in these lists are not stored in memory by DTCMGRUI but are requested one by one from DTCMGRIO through RL Switch. Every time the user wants to add, delete, or modify an item or scroll up or down the list, the whole list is reset and orders are sent to DTCMGRIO to reread the displayed part item by item (Fig. 9). This mechanism is used because each record in the list may be quite long and lists may have many records, so the amount of memory required to load all the items at once in memory and to have Microsoft Windows handle the list would be too large. Moreover, in remote mode, if the amount of information requested at one time is too large, the time between the start of a function and the function's actually becoming active would be too long. With this principle, in remote mode, each item is displayed as soon as it arrives, so it seems to be faster, and in any event, a maximum of 13 items will be transferred even if the list is 512 items long.

\section{Support Tool Integration}

Support engineers sometimes need to run special appli-

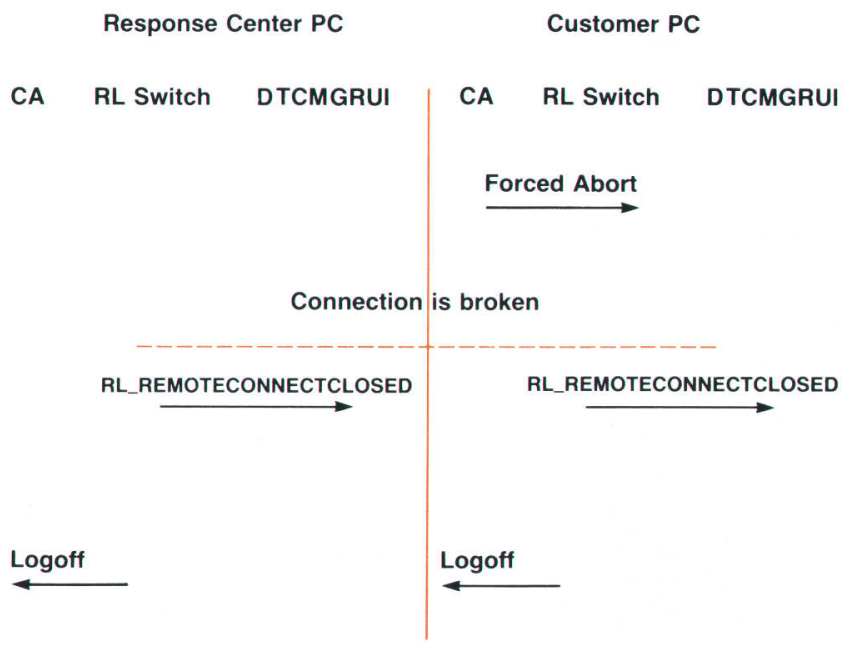

Fig. 8. Messages exchanged for a customer-forced abort or a modem line failure. 
cations-for example, a light formatter for the dump files coming from the DTC. These applications must be integrated into the HP OpenView DTC Manager, even though they were not defined at the time the HP OpenView DTC Manager code was written. To accommodate this need, hooks were implemented in DTCMGRUI and DTCMGRIO so that these applications can be added dynamically and integrated into the HP OpenView DTC Manager.

Except for some special procedures during initialization and shutdown, a DTC Manager application is a normal Microsoft Windows application that doesn't need to deal with HP OpenView Windows. Like the DTC Manager, DTC Manager applications are structured in two parts, one for the user interface and one for everything dealing with the files and the DTC (the second part is referred to here as the I/O part of the application). This allows them to be used in remote mode as well.

The structure of the solution is shown in Fig. 10. The DTC Manager application is accessed through a menu in the Tools subsection of the HP OpenView main menu. The Tools menu and its submenus are added by DTCMGRUI during the initialization phase when the DtcManagerApp section is found in the WIN.INI file.

When the DTC Manager tool (or application) needs to be used, the user selects the application from the Tools menu. DTCMGRUI receives the request from HP OpenView Windows and sends a message to DTCMGRIO requesting it to spawn the I/O part of the application. All HP OpenView DTC Manager menu items are grayed. DTCMGRIO spawns the I/O part of the application, passes the RL Switch window handle to it, and replies to DTCMGRUI that the spawning was successful. With the reply, it sends the handle of the I/O part of the application.

DTCMGRUI spawns the user interface part of the application and passes to it the handle of the $\mathrm{I} / \mathrm{O}$ part of the application and the handle of RL Switch. The modules of the application can now exchange messages directly through RL Switch using the RL_SENDDATA message.
When the application is no longer needed, the user selects a close function in the user interface part of the application, which notifies DTCMGRUI that it wants to quit. It does not quit yet. DTCMGRUI sends a request to DTCMGRIO to kill the I/O part of the application. DTCMGRIO kills the I/O part of the application and notifies DTCMGRUI. DTCMGRUI then kills the user interface part of the application and reenables the HP OpenView DTC Manager menu items.

The advantages of this solution are:

- The DTC Manager application modules are Microsoft Windows modules, not HP OpenView Windows modules. The DTC Manager application menu item will appear only if the WIN.INI file contains a DtcManagerApp section.

- The DTC Manager application code is loaded only when needed, so no memory is allocated to it while the HP OpenView DTC Manager is processing a management function.

- The DTC Manager applications don't have to deal with HP OpenView Windows, so they are independent of HP OpenView Windows and HP OpenView DTC Manager releases.

The DtcManagerApp section of the WIN.INI file has the following syntax:

\section{[DtcManagerApp] \\ App1 = Appli1 , APPUI.EXE, APPIO.EXE}

where App1 is the name of the DTC Manager application, Appli1 is the name of the menu item to be added to the Tools menu, and APPUI.EXE and APPIO.EXE are the names of the application's EXE modules. It is mandatory for these files to be in the subdirectory EXE of the DTC Manager data base. During the initialization process, DTCMGRUI looks in the WIN.INI file for the DtcManagerApp section. If it exists, the Tools menu is added to the HP OpenView menu bar. For each valid entry in this section, DTCMGRUI adds the

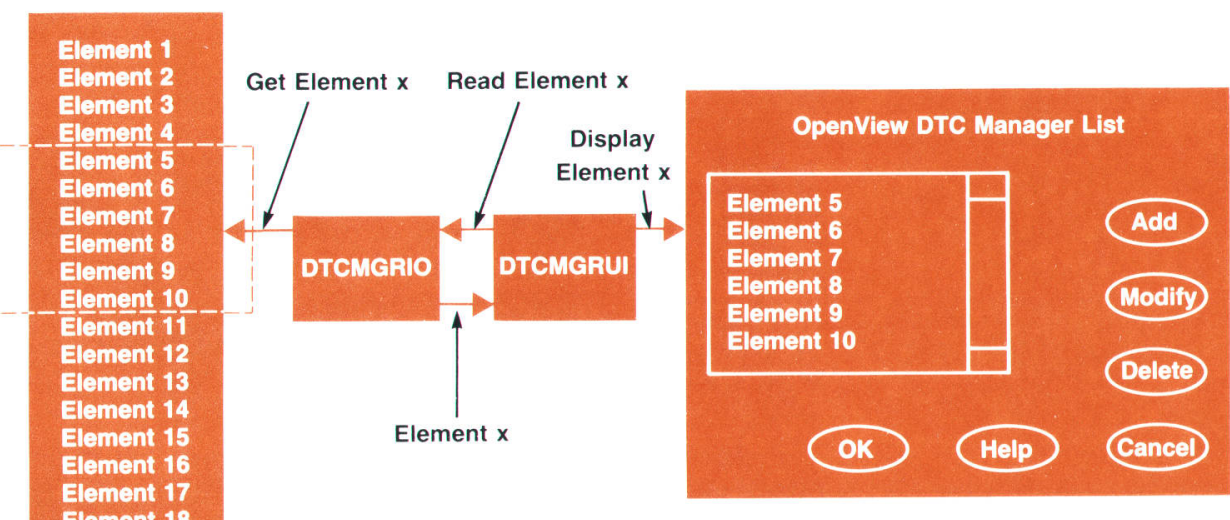

Fig. 9. HP OpenView DTC Manager lists are dialog boxes that contain list boxes that are managed by DTCMGRUI instead of Microsoft Windows. The items in the list are requested one by one from DTCMGRIO (through RL Switch). 
corresponding menu item in the Tools menu.

\section{HP OpenView DTC Manager Data Base}

The HP OpenView DTC Manager does not use the file access facility provided by HP OpenView Windows for three reasons. First of all, the file access facility provides single-key indexed file access and the HP OpenView DTC Manager would have needed multikey access. Second, the configuration data for a DTC can be very large and retrieving it via HP OpenView file access would have drastically slowed the initialization of a remote access if the user chooses to transfer the HP OpenView network topology map. Finally, not using HP OpenView file access means that the DTCMGRIO and PCMPRMP modules can be Microsoft Windows applications and not HP OpenView Windows applications, so that even if HP OpenView Windows is inactive, they are always active and ready to receive DTC events and service DTC download and upload requests. This allows the user to free memory by closing HP OpenView Windows to run another Microsoft Windows application while the PC continues to serve DTC-triggered management functions.

The HP OpenView DTC Manager data base takes advantage of the MS-DOS ${ }^{\circledR}$ directory hierarchical structure and stores data within a tree of subdirectories of the DTCMGR directory. The DTCMGR directory with its subdirectory tree is created at installation time in the directory specified by the user; the default is the root directory. The user may not specify more than one level of directory-for example, C:MYDIR. In this case the data base will be installed in C:MYOIRIDTCMGR.

The following list illustrates a typical HP OpenView DTC Manager data base containing a DTC named DTCNAME on the HP OpenView map, loaded with two serial interface cards in slots 0 and 1, one X.25 card in slot 4, and slots 2 , 3 , and 5 empty. The DTC code and configuration have been downloaded and X.25 protocol has been started on the $\mathrm{X} .25$ card but the PAD support protocol has not. In this list, lowercase is used for files and uppercase for directories:

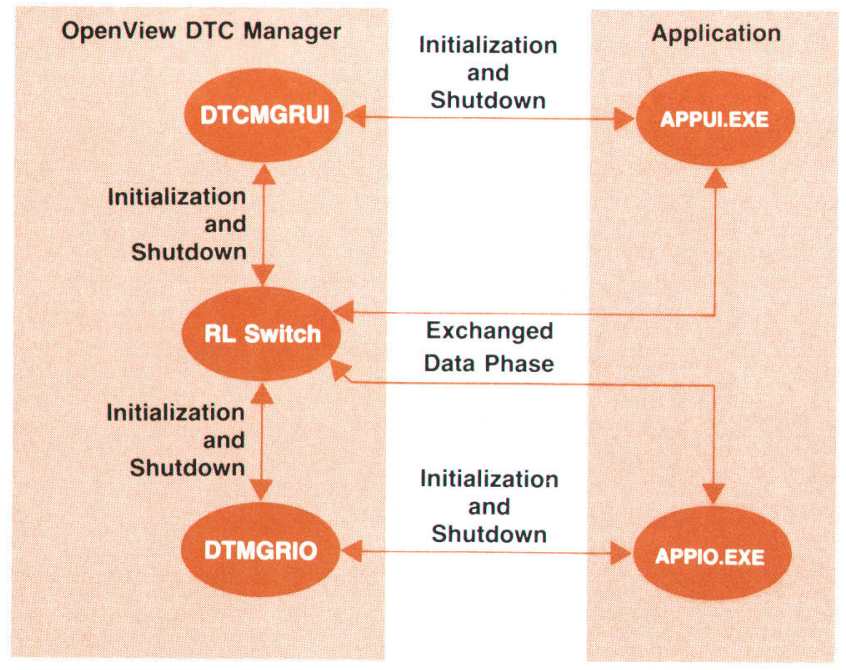

Fig. 10. Integration of applications such as support tools into the HP OpenView DTC Manager.

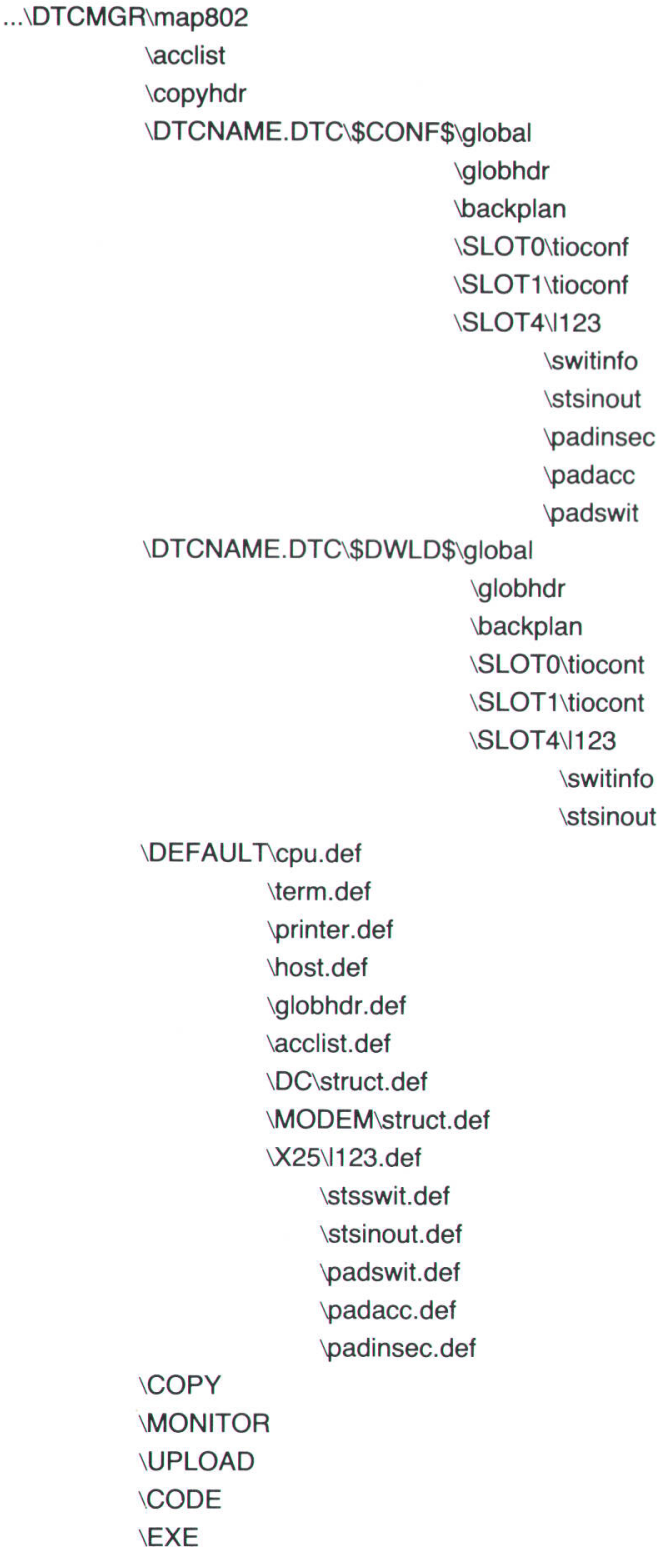

The file map802 contains one entry per configured DTC containing: the DTC name (e.g., DTCNAME), the DTC's current LAN (IEEE 802.3) address, the downloaded LAN address, and the DTC's LAN node name. It is updated and used by the DTCMGRIO module. Each time a DTC is created, its HP OpenView map name is written in this file with the two LAN addresses equal to 0 . When the user saves a configuration, DTCMGRIO gets the LAN address contained in the DTC CPU configuration and puts it in the current LAN address in the map802 file. When the DTC requests a download, DTCMGRIO copies the current LAN address into the downloaded LAN address.

The file acclist is the security list file, which is downloaded to all the configured DTCs.

The DTCNAME.DTC subdirectories contain the DTC configuration data. They describe a DTC and consist of up to three subdirectories: \$CONF\$, \$CONF\$.TP, and \$DWLD\$.

The \$CONF\$ subdirectory contains the DTC off-line con- 
figuration files. This configuration will be downloaded into the DTC on the next download operation. The contents of this directory and its associated subdirectories are modified when the user selects the Configure menu item. The \$CONF\$ subdirectory consists of three files and several subdirectories, one for each configured card of the DTC. The file backplan stores the information needed to display the backplane of a DTC. It contains 56 bytes:

- Byte 0: DTC Type. This is for future use, if we need to distinguish between multiple types of DTC.

ayte 1: Verify Flag. Indicates whether the configuration associated with this backplane has been verified.

- Six records of 9 bytes each, one record per card. Record 0 is for card 0, and so on. Each record contains the card type (empty, direct connect, modem connect, or X.25), and the port types (terminal, printer, or host) for ports 0 to 7 .

The file global contains the configuration of the CPU board (node name, IP address, logging classes, user prompt, welcome message). The file globhdr contains the LAN address of the DTC.

Each configured card has a SLOTx subdirectory, where $x$ is the card number (from 0 to 5 ). The files contained in each SLOTx subdirectory completely describe the card. The number of files and their contents depend on the card type. If the card is an 8-port direct connect card, its SLOTx subdirectory contains the file tioconf, which stores the configurations of ports 0 to 7 . If it is a 6-port modem connect card, its SLOTX subdirectory contains the file tioconf, which stores the configurations of ports 0 to 5 and two dummy configuration blocks. If the card is an X.25 card, its SLOTx subdirectory contains six files:

- 1123 stores level 1, level 2, and level 3 configuration data and the permanent virtual circuit list used by X.25.

- switinfo is a list that stores the host resolution table (system-to-system switching information) used by X.25.

- stsinout is a list that stores the LUG table (system-to-system local user group) used by X.25.

- padinsec is a list that stores the PAD security table (PAD incoming security) used by PAD support.

vadacc is a list that stores the PAD device table (PAD access) used by PAD support.

- padswit is a list that stores the PAD switching table (DTC PAD switching information) used by PAD support.

The \$CONF\$.TP subdirectory contains the temporary configuration files. This directory is used for temporary storage of the modifications when the user is in the off-line configuration function. The $\$ C O N F \$$.TP subdirectory is created by DTCMGRIO when the user starts modifying a DTC configuration. It has the same structure as the \$CONF\$ subdirectory.

The \$DWLD\$ subdirectory contains the downloaded configuration files. This is the image of the DTC configuration data. This set of files is updated during dynamic configuration functions. The \$DWLD\$ subdirectory is created by DTCMGRIO when the user creates a new DTC. Upon successful completion of a download operation DTCMGRIO will copy files from the \$CONF\$ directory into the \$DWLD\$ directory, which keeps an exact image of the configuration data downloaded to the DTC. When dynamic changes are done using the Set Parameters menu item, only the data con- tained in this directory is updated unless the user requests that the modifications be copied to the off-line configuration as well.

The COPY subdirectory is created by DTCMGRIO when the user selects the COPY function (in the configuration menu) for the first time. Thereafter, it is never removed, allowing the user to make several copies of the same item using the PASTE function. The file ..IDTCMGRicopyhdr contains the type of item copied. The type can be DTC (the entire DTC configuration), SIC-DC (direct connect card), SICModem (modem connect card), SSIC (X.25 configuration), Term (terminal configuration), Printer, or Host. This file is checked when the PASTE function is executed, since the COPY action and the PASTE action must be consistent. Then, according to the type of item, the subdirectory COPY is filled with all or part of a \$CONF\$ subdirectory.

The DEFAULT subdirectory contains all the default values for the configuration of a DTC. It has three subdirectoriesDC, MODEM, and X25 - and six default files, which contain the default configurations of each type of card in the DTC. The DC and MODEM subdirectories both have a file called struct.def ( 9 bytes), which contains the default structure for each card type and port type. The X25 subdirectory contains the default files to be used for an X.25 card.

The UPLOAD subdirectory receives the files containing upload data. These files are named depending on what kind of upload data they contain. DTCNAME and HOSTNAME are the names used by the user on the HP OpenView map.

The MONITOR subdirectory contains event logging files and trace files.

The CODE subdirectory contains the code files to be downloaded to the DTC.

The EXE subdirectory contains all the executable files of the HP OpenView DTC Manager and some support tools.

\section{Memory Organization}

The PC memory organization was one of the most difficult challenges of this project. The goal was to have the HP OpenView DTC Manager run on an HP Vectra ES/12 personal computer equipped with a $2 \mathrm{M}$-byte additional memory board (the HP 45944A Vectra ES expanded memory $\operatorname{card}^{6}$ ).

Microsoft Windows can run in two different modes: large frame or small frame. In small frame mode, the memory area from C8000 to EFFFF is used to map expanded memory. The data segments of Windows applications, Windows libraries, and applications' dynamic libraries are loaded into conventional memory, that is, below 640K. This optimizes the use of expanded memory, but it uses a large amount of the shareable memory and minimizes the parallelism of applications.

In large frame mode, Microsoft Windows sets what is called a bank line, specifying how much space will be used in the $256 \mathrm{~K}$-to-640K slot for expanded memory mapping. Once this line is chosen, everything below the line (from 0 to the line) is considered shareable memory (called nonbankable memory, below-the-line memory, or global memory). Everything above the line (from the line to $640 \mathrm{~K}$ ) is used for page banking (called bankable memory or abovethe-line memory). In this model the applications' data segments and dynamic libraries and the Windows libraries 
are banked, meaning that they are loaded in expanded memory.

The advantage of running in large frame mode is that it frees a lot of the conventional memory, thereby increasing the parallelism of applications. The disadvantage is that it uses a lot of expanded memory because Microsoft Windows duplicates some Windows library segments in every bank and the smallest application will take no less than $112 \mathrm{~K}$ bytes of expanded memory. Moreover, when an application needs more pages, Microsoft Windows will allocate pages to this application until it reaches the maximum number of pages that can be banked simultaneously. Thereafter, Microsoft Windows is not able to free these pages for other applications.

Code and resources (dialog box descriptions, text, etc.) are always banked in expanded memory whatever the mode.

The HP OpenView DTC Manager needs Microsoft Windows to start in large frame mode, because in small frame mode it runs out of global memory. Depending on the memory setting of the PC, Microsoft Windows/286 2.1 decides at start time in which mode it is going to run. Some of the criteria are:

- The amount of conventional memory left when Microsoft Windows is started. This depends on how many drivers are present and whether HIMEM is in use (the HIMEM driver saves $64 \mathrm{~K}$ bytes of conventional memory).

- The version of the expansion memory driver available. This should be EMS 4.0 to be in large frame mode.

- The type of expansion board used. It should support EMS 4:0 to be in large frame mode.

- The amount of expanded memory available.

- Whether backfilling is in use. It should be in use to be in large frame mode. Backfilling is the ability to replace the upper 256 or $512 \mathrm{~K}$ bytes of the CPU memory by an equivalent amount on the expansion memory board. This is mandatory to enable windows to set the bank line (also called the EMS line) below 640K bytes. In this situation, the memory from 0 to the EMS line is considered to be shared, and the memory from the EMS line

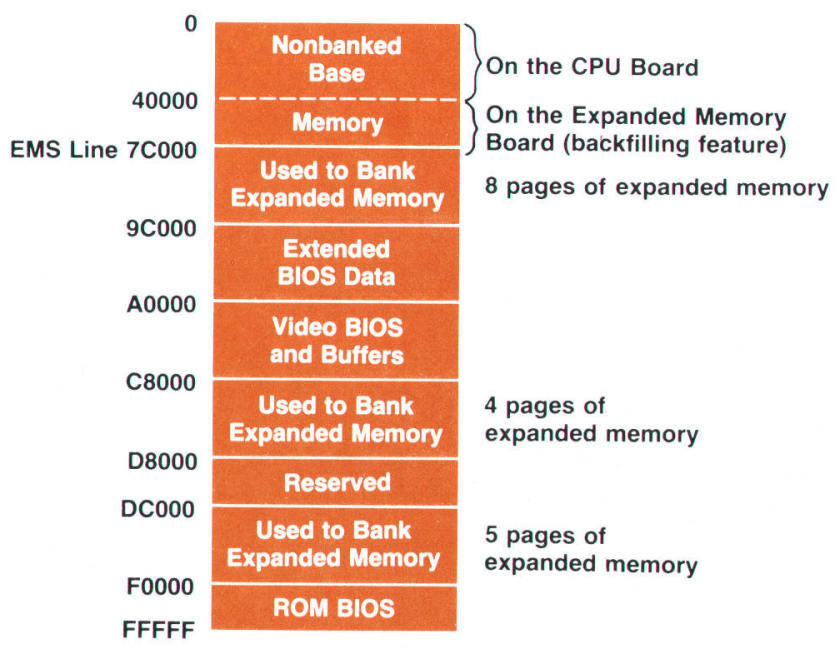

Fig. 11. HP Vectra ES personal computer memory map for the HP OpenView DTC Manager. to $640 \mathrm{~K}$ is used to bank expanded memory.

Taking all these parameters into account, the memory organization shown in Fig. 11 allows the HP OpenView DTC Manager to run correctly.

\section{Summary}

A new generation of HP 3000 computers needed a new generation of network management. The HP OpenView DTC Manager provides easy-to-use graphical interfaces, allowing the user to have comprehensive knowledge of the network and its components.

The important new shared services provided by the DTC also needed a management station that was independent of the host. The HP OpenView DTC Manager workstation provides this. However, a host may continue to manage a DTC when it is not necessary to share its services.

\section{Acknowledgments}

The HP OpenView DTC Manager was a joint effort of HP's Information Networks Division (HP OpenView Windows and RL Switch/CA), Business Networks Division (PCMPRMP), and Grenoble Networks Division (DTCMGRUI and DTCMGRIO). We would like to take this opportunity to thank all the teams involved for their contributions. It was an outstanding example of teamwork among all of the marketing, QA, production, and lab departments.

\section{References}

1. M.J. Mahon, et al, "Hewlett-Packard Precision Architecture: The Processor," Hewlett-Packard Journal, Vol. 37, no. 8, August 1986, pp. 4-21.

2. J.R. Busch, et al, “MPE XL: The Operating System for HP's Next Generation of Commercial Computer Systems," Hewlett-Packard Journal, Vol. 38, no. 11, December 1987, pp. 68-86.

3. D.V. James, et al, "HP Precision Architecture: The Input/Output System," Hewlett-Packard Journal, Vol. 37, no. 8, August 1986, pp. 23-30.

4. S. Youssef-Digaleh and A. Garg, Network Management Architecture and Protocol, Hewlett-Packard internal publication, Revision 2.2, September 1986.

5. C. Brunet, et al, DTC Network Management Internode Specifications, Hewlett-Packard, October 1988.

6. G.W. Lum, et al, "Expanded Memory for the HP Vectra ES Personal Computer," Hewlett-Packard Journal, Vol. 39, no. 6, December 1988, pp. 57-63.

Microsoft and MS-DOS are U.S. registered trademarks of Microsoft Corp. 


\title{
Developing a Distributed Network Management Application Using HP OpenView Windows
}

\author{
Using concepts from the HP OpenView architecture and \\ the facilities provided by HP Openview Windows, network \\ management services and distributed applications were \\ developed for user feedback and validation of the \\ architecture.
}

by Atul R. Garg and Lisa M. Cole

T HE HP OPENVIEW NETWORK SERVICES MONITOR (OV/NS Monitor) provides network management functions for distributed HP 3000 computers. OV/NS Monitor is divided into two parts: the main application and the user interface. The main application resides on an HP 3000 computer that is designated as a management node and the user interface resides on an HP Vectra personal computer. The main application performs network management functions via the software residing on the HP
3000 computers designated as managed nodes. OV/NS Monitor is for internal use only and is not available as a product.

This article describes the approach used to develop the OV/NS Monitor network management application using some of the concepts from the HP OpenView architecture and the facilities provided by the HP OpenView Windows software. Many of the ideas and concepts used in developing this application are being incorporated in the develop-

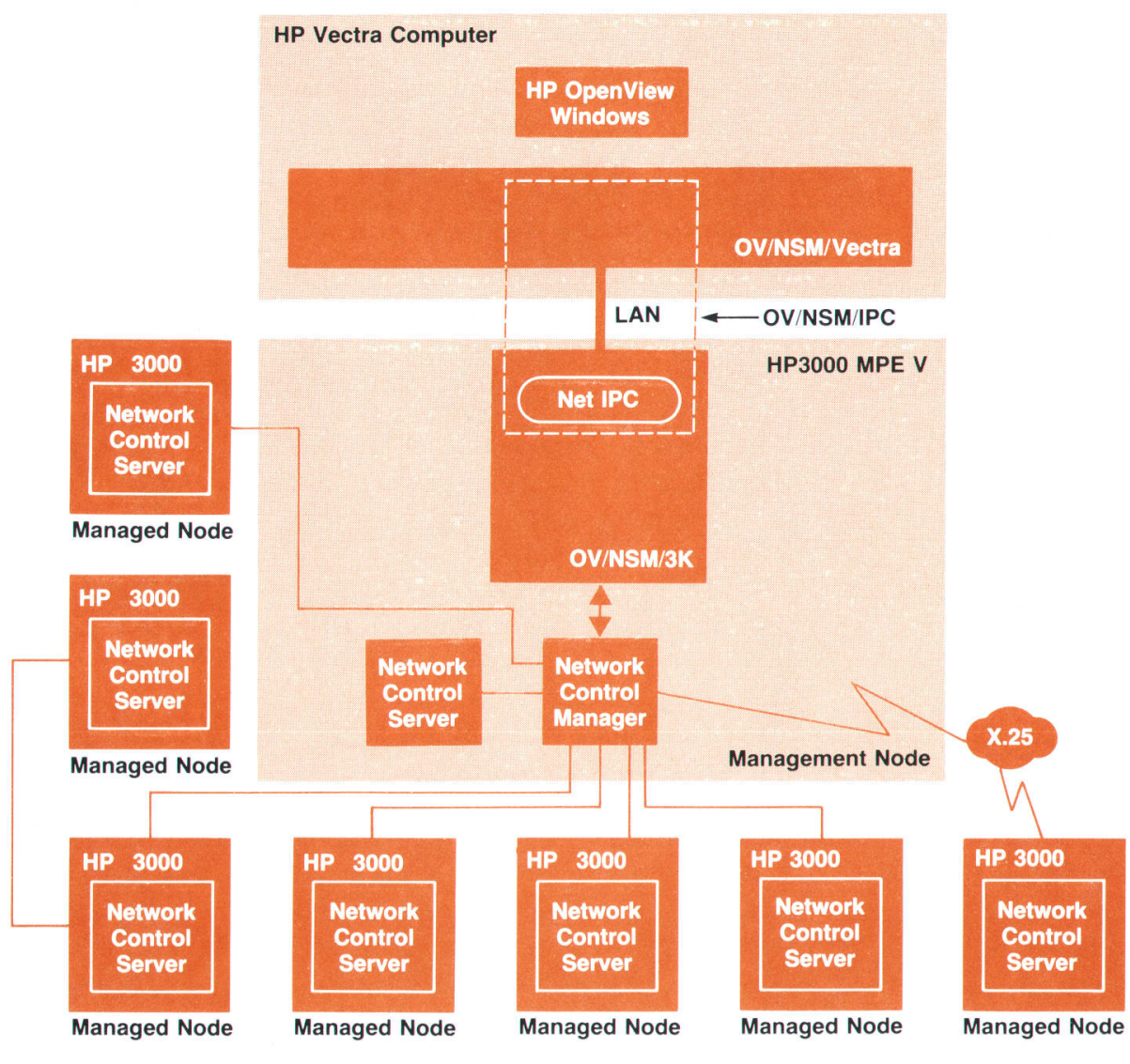

Fig. 1. The main components of the HP OpenView Network Services Monitor (OVINS Monitor). 
ment of other integrated and distributed network management products.

\section{System Overview}

A typical network consists of many nodes or computer systems. One (or more) of the nodes in the network is designated as the management node, and the other nodes in the network are referred to as managed nodes. The set of managed nodes can be connected to the network management node by a LAN, a set of point-to-point links, an X.25 link, or a gateway. Fig. 1 shows this configuration and some of the major OV/NS Monitor modules.

HP OpenView NS Monitor/Vectra (OV/NSM/Vectra). This portion of the OV/NS Monitor runs on the HP Vectra personal computer and uses the HP OpenView Windows utilities to interact with the user and IPC software to communicate with the portion of the OV/NS Monitor running on the HP 3000. OV/NSM/Vectra's function is to provide the user interface.

HP OpenView NS Monitor/3000 (OV/NSM/3K). This portion of the OV/NS Monitor runs on the HP 3000 computer that is designated as a management node. This portion of the OV/NS Monitor interfaces with OV/NSM/Vectra to service user requests, and it interacts with a module called the network control manager to retrieve network information from the managed nodes.

HP OpenView NS Monitor/IPC (OV/NSM/IPC). This software provides transparent interprocess communication between OV/NSM/Vectra and OV/NSM/3K. The IPC mechanism uses HP OfficeShare on the Vectra, and NetIPC ${ }^{1}$ on the HP 3000 to provide reliable communication between the HP Vectra personal computer and the HP 3000.

HP OpenView Windows. HP OpenView Windows runs on the Vectra and provides a set of utilities and functions to display application menus and interact with the user. It provides a common user interface for all network management functions.

Network Control Manager (NCM). The network control manager runs on the management node and handles communication with the network control server on the managed nodes.

Network Control Server. The network control server runs on the managed nodes and performs functions on the managed node in response to requests from the management node.

HP OfficeShare. This application provides communication services between HP Vectra personal computers and HP 3000 systems.

The architecture of the OV/NS Monitor permits multiple management nodes to control overlapping areas of the network-that is, two or more management nodes can receive data from the same node. The design also permits several Vectras to connect to the same management node and multiplex the communications in an orderly manner. Each Vectra can connect to a management node via a LAN, a direct connection, or a modem.

OV/NSM/Vectra requires a connection to the management node. If the user tries to perform a function that requires the use of the management node and the node is not already connected, a logon dialog box is displayed and the user is given an opportunity to connect to the manage- ment node. HP OpenView Windows does not provide any security of its own and expects each application to implement the required level of protection. In OV/NSM/Vectra, users are authenticated as they try to log on to the management node. As part of authenticating a user, each user is given a level of security clearance at the management node. Three capability levels are recognized: reader, writer, or super user. If a user does not have sufficient capability for a particular menu item it is not selectable. This is done by a Microsoft Windows concept called graying out (dimming) the menu item.

The following sections describe the overall design of OV/NSM/3K and OV/NSM/Vectra in more detail and provide some insight into the operation of these applications.

\section{HP OpenView NS Monitor/3000}

To provide an extensible architecture that enables new distributed management applications to integrate easily with HP OpenView NS Monitor, a modular approach was employed to design the OV/NSM/3K modules. For the highlevel design, HP Teamwork/SA was used to create data flow diagrams and to validate the consistency of these diagrams. For low-level design, finite state machines were used to design each individual module, and in some cases, state tables were used to settle several design issues. Since $\mathrm{OV} / \mathrm{NSM} / 3 \mathrm{~K}$ is modular, a message-based interprocess communication (IPC) mechanism was adopted because it proved to be more flexible and easier to use than other alternatives, such as procedural IPC. Pseudocode and text specifications were also used to help promote and explain the design and interfaces between the modules.

\section{Operation of OV/NSM/3K}

$\mathrm{OV} / \mathrm{NSM} / 3 \mathrm{~K}$ is started by running a stream job after the network control manager and network control server software have been successfully started. The stream job sets up some file equations and logging options, and then starts the Monitor process, which in turn starts other OV/NSM/3K processes. Once initialization is done, a message is printed on the operator console indicating success or failure of the start-up process.

OV/NSM/3K is stopped by executing a UDC (user-defined command) script. When this is done a HALT message is sent from the Monitor process to all of its child processes. Each process, after receiving the HALT message, is responsible for cleaning up before exiting.

Before any messages can flow between the Vectra and the management node, a connection must be established between OV/NSM/Vectra and OV/NSM/3K. A connection is initiated when the user logs on to OV/NSM/Vectra. OV/ NSM/Vectra formats the logon request and sends it to OV/ $\mathrm{NSM} / 3 \mathrm{~K}$. After validating the request, OV/NSM/3K sends a confirmation logon back to OV/NSM/Vectra that indicates the connection is established and more requests can be sent from the Vectra to the management node.

When the user invokes a network management service (e.g., Try Connection), the request is sent to $\mathrm{OV} / \mathrm{NSM} / 3 \mathrm{~K}$, which tries to establish a connection with the network control server on the targeted (managed) node. If the connection is successfully established, the request is sent to 
the network control server on the target node. After processing the request, the target node will send back a response which is forwarded to the user on the Vectra via OV/NSM/ $3 \mathrm{~K}$.

As shown in Fig. 2, OV/NSM/3K consists of five processes. The Monitor, Status, and Opt Logger processes are required to be running at all times. The Diag and Config processes are started when the user logs in and are terminated when the user logs off. All of these processes use a message-based IPC mechanism to communicate with each other.

\section{Monitor Process}

The Monitor process is the parent process of the other processes in OV/NSM/3K. It is started by the stream job described earlier. During initialization it starts the Status and the Opt Logger processes. The Monitor's primary function is to receive requests from any OV/NSM/Vectra trying to establish a dialogue with the Config or Diag processes. After verification of the user password, the Monitor process spawns the Config or Diag processes and passes the information to them so that they are able to communicate with OV/NSM/Vectra. Fig. 3 shows the data flows for setting up a connection to a Config process. If there is a status or configuration change made, the Monitor process will broadcast the change to all the active Diag processes. Regardless of the number of Vectras running OV/NSM/Vectra, there is only one Monitor process running at all times.

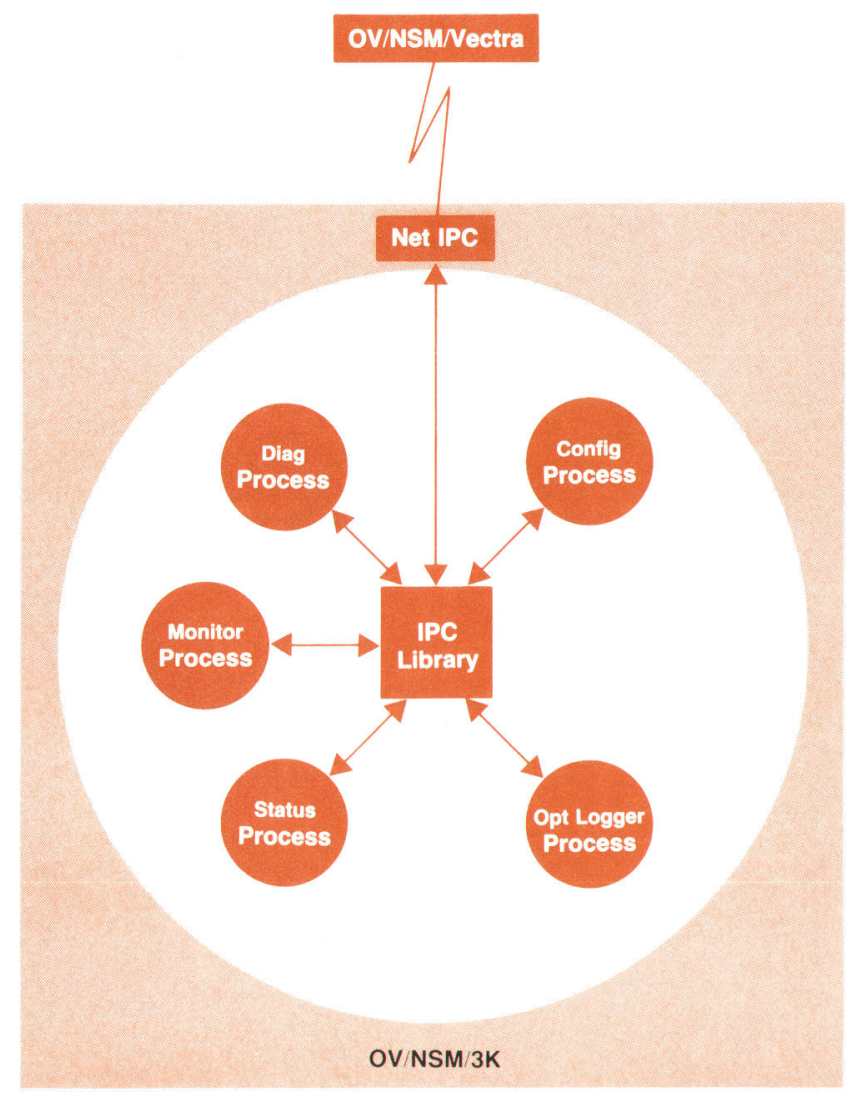

Fig. 2. The main components of the HP OpenView Network Services Monitor/3000 (OV/NSM/3K).

\section{Status Process}

The Status process has two principal tasks: maintaining the state of each object that can be displayed on the network topology map and acting as a centralized collector of remote messages. The Status process changes the state of an object based on events received from local or remote nodes.

Anytime a node changes state, the Status process sends a message to OV/NSM/Vectra (via the Monitor process) and writes a message to the event log. The Status process also conveys the state of all nodes to a Diag process on request.

The Status process's second task of acting as a centralized collector of remote logging messages involves logging only those messages that it considers critical enough to be of interest. The Status process writes these messages to log files, which are protected from being written to by any other process. Some messages that are sent to these files are generated by the Status process itself in response to time-outs or other local events.

\section{Opt Logger Process}

The Opt Logger is the process that collects network performance data. Its basic functions are to request the network control server on a target node to start a data collection run, stop a running data collection run, and collect the data produced by a data collection run. A data collection run is both the process of collecting network performance data and the storing of that data in a TurboImage data base.

\section{Diag Process}

The Diag process provides the diagnostic functions for troubleshooting a specific network problem. The Diag process is started by the Monitor process when a valid logon

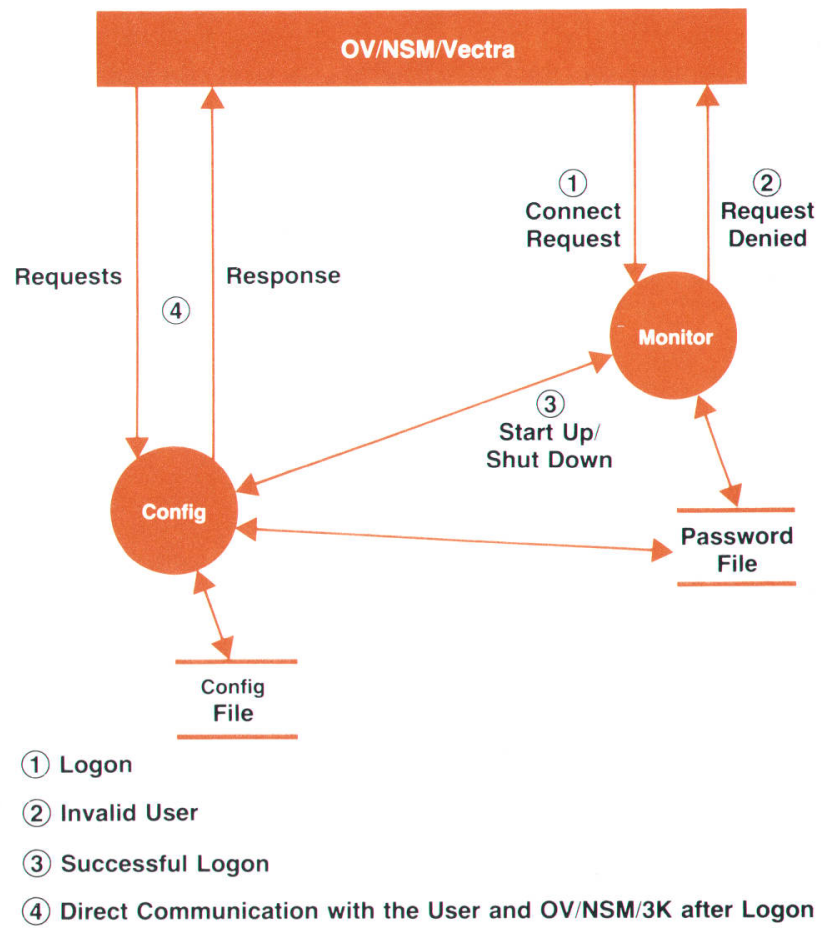

Fig. 3. OVINSM/3K Monitor process interactions with the Config process during initialization and shut down 
request is received from OV/NSM/Vectra. If the Diag process does not encounter an error, it will send a logon confirm message to OV/NSM/Vectra, thus establishing a connection. After this, all messages to or from OV/NSM/Vectra will go through the Diag process.

There can be multiple instances of the Diag process running at one time- one per Vectra connection. Since the Diag process is started by the Monitor process, it can also be brought down by the Monitor process via the HALT message. The Diag process can also terminate if it detects its connection with OV/NSM/Vectra is broken or it receives an EXIT message from OV/NSM/Vectra when the user logs off.

\section{Config Process}

The Config process implements OV/NS Monitor configuration functions that include user management and network topology file management.

The Config process is started up by the Monitor process when it receives a valid logon with a request to start the Config process. Only one Config process can be active on the management node at a time. The Config process can be shut down in the same manner as the Diag process.

\section{Interprocess Communication for OV/NSM/3K}

All OV/NSM/3K processes use a set of IPC library routines to send and receive messages to and from each other, to set up a timer that causes a process to be notified when the timer expires, and to interface to NetIPC for communication between QV/NSM/3K and OV/NSM/Vectra and the communication ports on the HP 3000 . The IPC library provides a uniform IPC interface and centralizes the IPC related code. Collecting all the IPC in one place enables the underlying IPC mechanisms to be changed without affecting the other processes and having the same IPC interface in all modules made it possible to integrate the OV/NSM $/ 3 \mathrm{~K} \bmod -$ ules with little difficulty. The IPC library also minimizes the processing overhead that takes place on the Vectra side of OV/NS Monitor. This objective is achieved by using a fixed-size header in every message. Thus, the address of where function dependent data begins in a message can be easily determined by adding the fixed number of bytes representing the size of the message header to the address of the first byte of a message.

\section{HP OpenView NS Monitor/Vectra}

The OV/NSM/Vectra software is an integral part of OV/NS Monitor, yet it is distinctly different from the OV/NSM/3K portion of OV/NS Monitor in a few key respects. First, OV/NSM/Vectra runs on a single-user personal computer running MS-DOS ${ }^{\circledR}$, Microsoft ${ }^{\circledR}$ Windows, and HP OpenView Windows. OV/NSM/3K operates and was developed on a multiuser HP 3000 business computer running the MPE V operating system. Second, OV/NSM/Vectra is the user interface portion of the OV/NS Monitor, while the $\mathrm{OV} / \mathrm{NSM} / 3 \mathrm{~K}$ software is used to monitor and control the nodes being managed by the user on the Vectra. Because of these differences, distinct requirements were developed for the OV/NSM/Vectra software.

The basic strategy used to provide for extensibility and

Microsoft and MS-DOS are U.S, registered trademarks of Microsoft Corporation. changes resulting from user feedback was to modularize the architecture based on the natural divisions present in OV/NSM/Vectra applications. While doing this, the constraints imposed by both Microsoft Windows and HP OpenView Windows had to be accommodated.

$\mathrm{OV} / \mathrm{NSM} / \mathrm{Vectra}$ is required to handle two main interfaces: the user interface and the interface to HP 3000 software. The interface between the Vectra software and the HP 3000 software is message-based and the interface between OV/NSM/Vectra and the user is arranged in terms of dialog boxes. Thus, the basic design clusters the parts for a particular activity into one module to create a specific interface to the user. This includes the dialog box and its associated resources, the dialog box procedure, and the message interface to the HP 3000. This arrangement is shown in Fig. 4. Modules are as independent as possible, and each module assumes very little of its environment. Global state data is reduced to a minimum and, where applicable, is manipulated by access procedures rather than directly by the various modules.

To help meet the goal of timeliness, code reuse was employed and implemented in two ways: linkable libraries and extensive use of code templates. Code that is commonly used by many modules was put into linkable libraries to reduce code space use. For modules that were similar in structure but differed slightly in specifics, templates were created. Dialog box procedures are the best examples of this. All the OV/NSM/Vectra dialog box procedures have the same basic structure, differing only where required to perform the unique task or service provided by that box. This increased code size but leveraged large amounts of design and coding effort.

Wherever possible, an attempt was made to exploit the features provided by Microsoft Windows. For example, Microsoft Windows provides natural and easy ways of creating object classes called subclasses. OV/NSM/Vectra used this feature extensively to leverage the amount of original coding that needed to be done for the user interface.
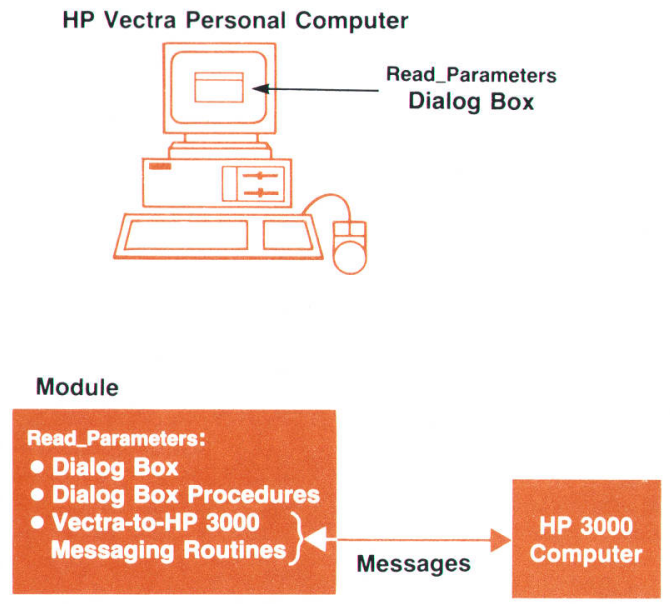

Fig. 4. The design strategy for modularization in OVINSMI Vectra for a particular user activity. The dialog box, the dialog box procedures, and the Vectra-to-HP 3000 routines are combined into one module. 


\section{OV/NSM/Vectra Structure}

Each of the OV/NSM/Vectra applications consists of three separate processes: OV/NSM/VectraRun, OV/NSM/VectraDraw, and OV/NSM/VectraAdmin. This set of processes, together with the OV/NSM/3K processes, provides the OV/NS Monitor network management facilities.

The division of functionality between the three processes is based on the guidelines recommended in the HP OpenView Windows Developer's Kit Writer's Style Guide. A common application structure for developing the three processes was created without regard for the functional differences between them. There are three areas of interaction in this common application structure with which OV/NSM/ Vectra is concerned: interacting with the user, interacting with the management node, and interacting with the network.

\section{Interacting with Users}

Interacting with the user requires OV/NSM/Vectra to interface with both Microsoft Windows and HP OpenView Windows, with the majority of the interaction taking place directly with Microsoft Windows.

Microsoft Windows Interface. Most of the OV/NSM/Vectra tasks are simple Microsoft Windows applications. They use the intrinsics provided by the Microsoft Windows 2.0 Software Developer's Kit, and the standard Microsoft Windows objects such as dialog boxes, menus, and icons.

The most common operation performed by OV/NSM/ Vectra is to solicit user input and display user output using dialog boxes. The dialog box is used as a unit of modularization in OV/NSM/Vectra. That is, a dialog box, along with its dialog box procedure, IPC routines, and printing routines, forms a dialog box module. Each dialog box module is responsible for handling the requests and responses between the Vectra and the management node. It does this using the services of the transaction manager module and the IPC interface, which are described later. Fig. 5 shows this structure.

Sometimes there was a need to modify the default behavior of Microsoft Windows as in the case of the predefined window classes called controls. An example of a control is a single-line edit field that allows an application to display information to the user and receive input of any type from the user. The properties of controls can be changed by a process known as subclassing, which allows the application to inherit the current set of properties associated with a control and modify those properties to create a new type of control. An example of a subclassed control is the password subclass, which is a single-line edit control that allows the user to input only alphanumeric characters, the first of which must be an alphabetic character. This subclass does not echo the keyboard input to the user.

OV/NSM/Vectra uses subclasses frequently. The benefits of subclassing to OV/NSM/Vectra are twofold. First, OV/ NSM/Vectra adds syntax checking to all of its new controls. Thus, syntax errors are detected as soon as an incorrect character is entered rather than resorting to primitive, forms-based methods of reporting errors. Second, the use of subclasses allows OV/NSM/Vectra to add or remove functionality without having to create new controls from scratch, reducing the amount of time required for design, coding, and testing of new code.

Some of the features of Microsoft Windows did present large design and implementation problems during the development of OV/NSM/Vectra. One of these problems is that Microsoft Windows provides no true communication capabilities of its own, nor does it provide an interface to any of the commonly accepted methods of communication for personal computers (e.g., NetIPC or NetBIOS). Integrating one of these communication mechanisms into a Microsoft Windows application proved to be quite a challenge and is described in more detail in the section on interacting with the network.

HP OpenView Windows Interface. To operate in the HP OpenView Windows environment with other applications, there are certain standard Microsoft Windows functions that applications ask HP OpenView Windows to perform on their behalf, such as adding menus to the menu bar. This gives HP OpenView Windows some measure of control to present a consistent look and feel to the HP OpenView environment. Applications use the intrinsics provided by the HP OpenView Windows developer's kit to access these normal Microsoft Windows features, as well as some administrative tasks required to operate as an HP OpenView Windows application.

HP OpenView Windows applications are unlike normal Microsoft applications in some respects. One difference is that the main window for an application (the window most applications create when they come alive) is invisible. This window has zero coordinates and is used for taking advantage of the messaging facilities of Microsoft Windows. It is known as a communication window and is created by making a call to HP OpenView Windows. The actual window displayed on the screen with the map and menus is wholly owned and operated by HP OpenView Windows. Since Microsoft Windows associates a message queue with an

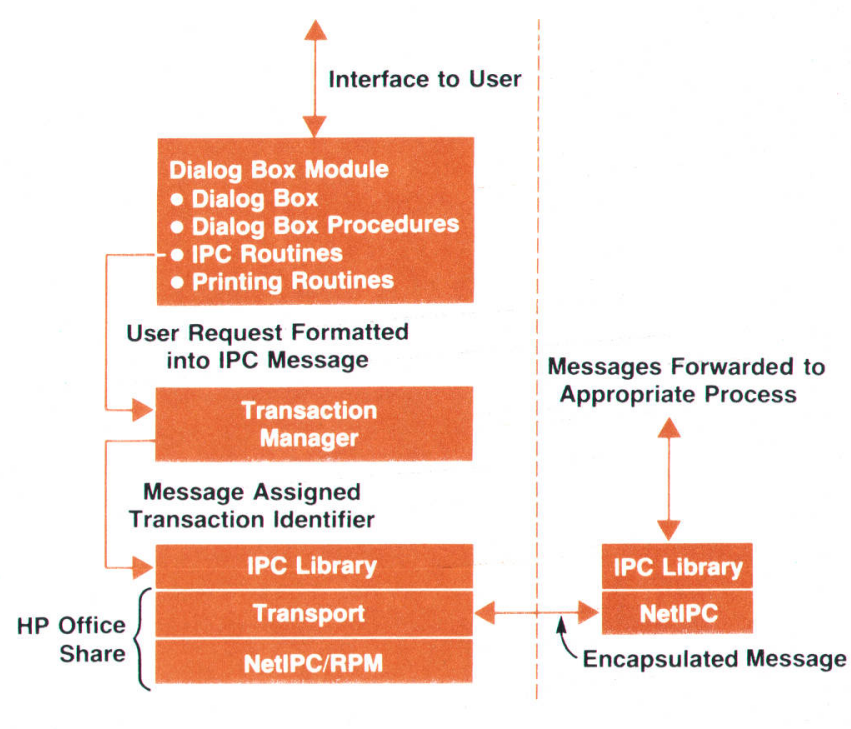

OV/NSM/Vectra Workstation Network Management Node

Fig. 5. The modules involved in providing interaction with the user and interaction with the network in the OVINSMI Vectra software. 
application's main window and posts all messages destined for an application to this queue, all messages resulting from user interaction with the map and menu items are received by HP OpenView Windows. HP OpenView Windows then forwards messages that arrive in its queue to the appropriate application's communication window for subsequent processing.

\section{Interacting with the Management Node}

The bulk of the OV/NS Monitor resides on the HP 3000 management node. The OV/NSM/Vectra applications establish communication with the management node using TCP/ IP via the NetIPC interface. All communications to and from the management node are in one of the predefined OV/NS Monitor message formats. The normal mode of operation is request/response oriented, with all requests being initiated from the OV/NSM/Vectra side. These requests are received and processed by $\mathrm{OV} / \mathrm{NSM} / 3 \mathrm{~K}$ and the results are formatted and returned to OV/NSM/Vectra.

This appears to be straightforward. However, a fundamental design feature of OV/NSM/Vectra is that multiple requests can be outstanding on the management node. OV/NSM/Vectra cannot, therefore, block on any individual reply. A method of pairing requests with responses and associating these request/response pairs with their originating dialog boxes had to be developed. To complicate matters further, there are a few asynchronous event messages that originate on the management node, such as the notification of the shutdown of the OV/NSM/3K software. These asynchronous messages have no associated dialog box, but need to be handled in as efficient a manner as any other response. Satisfying these requirements placed some additional constraints on the design of the network interface.

\section{Interacting with the Network}

OV/NSM/Vectra handles the receipt and sending of packets across the network by encapsulating network packets as Microsoft Windows messages to a dialog box procedure. This interface is defined and supported by two modules: the IPC library and the transaction manager shown in Fig. 5.

The IPC library is a thin layer of software that interacts with the HP OfficeShare communications software. HP OfficeShare is divided into two layers: the transport layer and the NetIPC/RPM layer. The transport layer supports either an HP ThinLan link or a serial link. Based on the physical connection between the Vectra and the management node, one of these transports must be loaded into memory by the user before running Microsoft Windows. Only one type of link can be loaded at a time, and it resides in Vectra EMS memory. ${ }^{2}$ OV/NSM/Vectra assumes nothing about the transport except that it expects it to be present. Since the transport software is sitting outside of Microsoft Windows, there has to be a way of requesting data to be sent on the link. This is provided by a dynamic library included in the NetIPC/RPM development package. This library provides a standard NetIPC interface for use with Microsoft Windows-based applications. This is the only communications interface OV/NSM/Vectra deals with.

Like the NetIPC interface library used by OV/NSM/3K, OV/NSM/Vectra's IPC library provides a set of procedures for accessing the NetIPC interface. These access procedures hide the complexities of connection establishment, termination, and other network operation from the rest of the application, thereby allowing them to make simple open, close, read, and write calls to the network.

The transaction manager is responsible for creating and destroying transactions associated with dialog boxes as well as distributing packets from the network along with a transaction identifier to the correct dialog box. Each dialog box procedure requests a transaction from the transaction manager when it has a request to send to the management node. The transaction manager associates this transaction, identified by a unique transaction identifier, with the dialog box handle. The packet is then sent to the management node. The response to this request is also identified by the transaction identifier. At some point the response packet is retrieved from the network by the IPC library module. It is given to the transaction manager module, which then looks at the transaction identifier and posts the message to the dialog box registered for this transaction. The dialog box procedure then processes the message as it processes all Microsoft Windows messages, completely unaware that the message actually came from the network.

\section{Combining the Interfaces}

When HP OpenView Windows is started from Microsoft Windows, the user is actually executing one of the HP OpenView Windows processes OVRun, OVDraw, or OVAdmin (see article on page 60). These processes will spawn all other Run, Draw, and Admin applications, respectively. For the OV/NS Monitor these include OV/NSM/VectraRun, OV/NSM/ VectraDraw, or OV/NSM/VectraAdmin. The list of applications that are spawned is kept in the Microsoft Windows initialization file WIN.INI along with the location of the user's default map, the NewWave help files ${ }^{3}$ used by all HP OpenView Windows applications, and the time and date localization information.

When actions performed by the user on the HP OpenView Windows map are of interest to OV/NSM/Vectra, HP OpenView Windows forwards a message to OV/NSM/Vectra's communication window. In the order received, these messages are appended to the queue and are processed, along with any other Microsoft Windows messages posted directly to the queue.

Once the user's selection has been passed to OV/NSM/ Vectra, HP OpenView Windows fades into the background and OV/NSM/Vectra makes calls directly to Microsoft Windows to display dialog boxes, solicit user input, and display results. OV/NSM/Vectra requests are packaged into IPC packets and sent to the management node. When the responses are returned from the management node, they are received by the HP OfficeShare transport. When the network polling mechanism in OV/NSM/Vectra discovers that a packet has been received from OV/NSM/3K, it copies the packet contents into its own data space. The transaction manager then reads the packet header to locate the transaction identifier to determine which dialog box should receive the packet. Once it knows where to send it, the transaction manager encapsulates the packet into a Microsoft Windows message and posts it to the dialog box that originated the request. Once this message is received by the procedure associated with the dialog box, the message is 
disassembled and its results are displayed in the dialog box display area.

If the transaction manager discovers, after reading the packet header, that the packet contains an asynchronous message rather than a response to an earlier dialog box request, the packet is encapsulated in a Microsoft Windows message and passed to the OV/NSM Vectra asynchronous message handler for further processing.

\section{Conclusion}

Developing the HP OpenView NS Monitor applications helped to identify the common functions that are required to provide a framework for the development of distributed and integrated network management functions. We found that it was essential to provide a common interprocess communication facility between the user interface, the management node, and the managed nodes. In addition, validation of users and their access rights, handling of events and status monitoring, and data storage and retrieval must be designed and developed uniformly to support the needs of all applications that are to be integrated.

\section{Acknowledgments}

We wish to acknowledge the technical accomplishments and contributions of the other members of the HP OpenView NS Monitor project team: Tin Phan, Chandramohan Thekkath, Kumar Vora, Richard Bogen, John Hardin, Wilson Ang, Lisa Gullicksen, and Jim Schnitter. Special recognition should also be given to Tony Ridolfo, the original project manager for the HP OpenView NS Monitor project. In addition, because of the integrated nature of the prototypes that were being developed, we would like to thank all the members of the HP OpenView Windows and HP OpenView core software development teams, as well as all the members of the network management product team for their valuable contributions, without which we could not have completed this project.

\section{References}

1. K.J. Faulkner, et al., "Network Services and Transport for the HP 3000 Computer," Hewlett-Packard Journal, Vol. 37, no. 10, October 1986, p. 14.

2. G.W. Lum, et al., "Expanded Memory for the HP Vectra ES Personal Computer," Hewlett-Packard Journal, Vol. 39, no. 6, December 1988, pp. 57-63.

3. V. Spilman and E.J. Wong, "The HP NewWave Environment Help Facility," Hewlett-Packard Journal, Vol. 40, no. 4, August 1989, pp. 43-47. 


\section{Authors}

\author{
April 1990
}

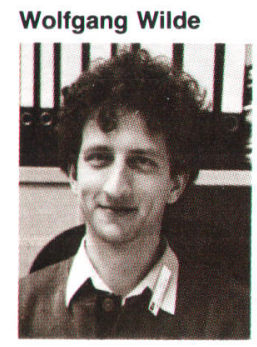

Wolfgang Wilde performed reliability engineering tests during development of the HP 1050 liquid chromatograph system. He is currently the quality engineering manager for HP's Waldbronn Analytical Divi sion. He earned a diploma (1986) in physics from the University of Mainz in West Germany, and joined $\mathrm{HP}$ in 1987 as a reliability engineer

\section{7 - LC Sample Injector/Autosampler -}

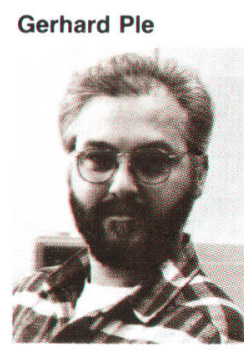

Gerhard Ple served as project leader for the autosampler for the HP 1050 liq uid chromatograph system and was responsible for $\mathrm{HP}$ 1050 firmware development. In a prior HP position, he developed electronic hardware and firmware for the HP 1090 autoinjector, autosampler, and pump configuration. Gerhard joined HP's Waldbronn Analytical Division in 1979 after receiving his electrical engineering diploma from the University of Karlsruhe that year. He was born in Neustadt, West Germany, and currently resides in Karlsruhe. ence. He also is a graduate of Fachhochschule Ulm (1974) with a degree in engineering. A member of Gesellschaft für Informatik, Herbert's professional interests are centered around control systems and applications for analytical instruments. Born in

Stuttgart, he resides in Spielberg with his wife and two children. He enjoys tennis, skiing, and traveling.

11 _.C. Quality Engineering:

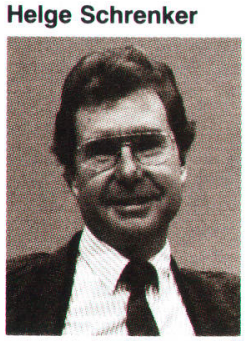

Helge Schrenker was HP's Waldbronn Analytical Division quality manager during development of the HP 1050 liquid chromatograph system. He joined HP in 1973 as a product marketing manager. In 1979, he became an R\&D group leader investigating flow control systems and doing systems integration for the HP 1090 high-performance liquid chromatograph. In 1983, he became a quality assurance manager. Helge earned an applied physics diploma (1967) from Kiel University. He has written several technical articles on $\mathrm{pH}$ measurement and control, concepts of fast-liquid chromatography, flow control in high-performance liquid chromatog raphy, and the effect of mobile phase preheating on liquid chromatography performance. His work has resulted in patents on a flow-controlled highpressure pump and a column thermostat for highperformance liquid chromatography. Before joining $\mathrm{HP}$, Helge worked in technical marketing support for Philips Electronic Industries, and served in the German Air Force. Born in Marbach, West Germany, he and his wife live in Karlsruhe. His hobbies and interests include photography, bicycling, environmental protection, and alternative traffic concepts.

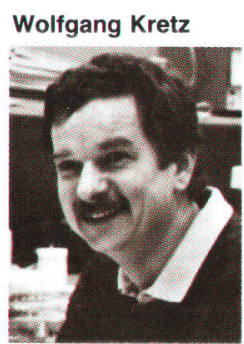

R\&D project engineer Wolfgang Kretz served as project leader for the mechanical design of the HP 1050 liquid chromatograph autosampler. He also was project leader for development of the autoinjector for the HP 1090 liquid chromatograph, de-

veloped improvements for the HP 79841A injector system, and served as a production engineer for the HP 1090. Wolfgang received his mechanical engineering degree (1972) from the Fachhochschule Konstanz, and an engineering diploma from the University of Stuttgart in 1978, the year he joined HP. Born in Buchheim, West Germany, Wolfgang now lives in Waldbronn with his wife and two children. His hobbies include photography, reading, hiking, and astronomy

\section{4 _ LC Solvent Delivery System -}

Klaus Witt

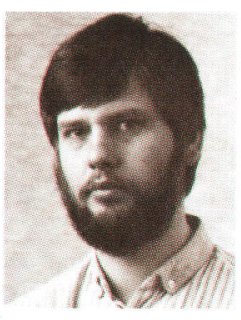

Klaus Witt contributed to the design of the pump driver and control system for the HP 1050 liquid chromatograph system. He is now a group manager within R\&D. He joined HP in 1979 as an R\&D engineer in the Waldbronn Analytical Division, shortly after

graduating from the Fachhochschule Osnabrueck with an electronics diploma. At HP, he helped design the digital servo chip for the high-performance liquid chromatograph. He is named an inventor in a patent application for a variable-stroke pump Born in Nedlin, Germany (now Poland), Klaus served 15 months in the West German Army, and now resides in Keltern, Germany, with his wife and two children. His hobbies include raising horses and horseback riding.

\section{Fred Strohmeier}

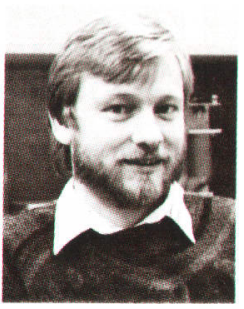
Mechanical engineering and hydrodynamics are the professional interests of Fred Strohmeier, section manager with HP's Waldbronn Analytical Division. He helped design the HP 1050 and HP 1090 liquid chromatograph systems, and is currently the project leader for the HP 1050 pumping system. Fred, who joined HP in 1979, is named an inventor in patent applications for a pumping apparatus to deliver liquid at high pressure and for a sample in jector for liquid chromatography. He received his engineering diploma in 1979 from the Fachhochschule Karlsruhe. Born in Baden-Baden, Fred re sides in Rheinmuenster, West Germany, with his wife and child. He enjoys jogging, canoeing, and reading

36 - LC Absorbance Detectors

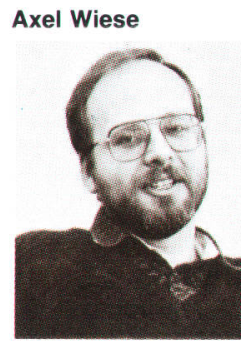

A year after earning his $\mathrm{PhD}$ degree in physics and physical chemistry in 1977 Axel Wiese joined HP's Waldbronn Analytical Divi sion. He developed the HP 79854A multiwavelength detector for the HP 1050 liquid chromatograph system. Before that, he de-

signed the HP 79881A filter photometric detecto and the HP 1046A fluorescence detector. Now a program manager, Axel has authored several tech nical papers in the field of microwave instrumentation. He resides in Karlsruhe, West Germany, and his hobbies include traveling and archeology.

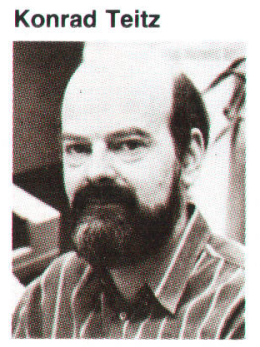
Project manager Konrad Teitz helped develop the HP 79853A variable wavelength detector, which is part of the HP 1050 liquid chromatograph system. In the past, he has developed other detection systems for the liquid chromatograph instruments produced at the Waldbronn Analytical Division. He joined HP in 1973 in Böblingen, West Germany, and is named an inventor in a patent concerning 
a new type of ultraviolet absorbance detector. His professional interests include electronic circuit simulation and EMC. Konrad, who received an engineering diploma in 1973 from the University of Karlsruhe, West Germany, was born near Lippstadt, and now resides in Karlsbad with his wife and son. He enjoys backpacking, photography, and amateur radio.

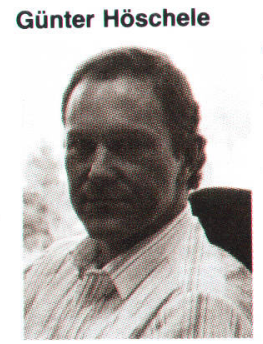

Günter Höschele developed data processing firmware for the HP 1050 liquid chromatograph system. He also helped develop the HP 1040A detector and HP 79881A detec tor data acquisition hardware and firmware Günter joined HP in 1979, two years after graduating from Stuttgart University with an electronics diploma. An R\&D engineer with HP's Waldbronn Analytical Division, his professional interests are fast data acquisition and processing. Born in Stuttgart, Günter currently resides in Langensteinbach, West Germany. He enjoys reading, high-fidelity music, and bicycle riding.

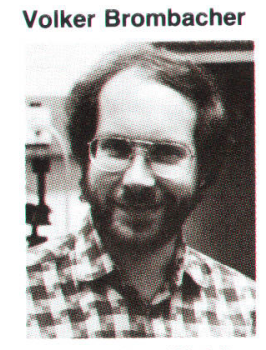

Volker Brombacher contributed to the development of the analog-to-digital converter for the multiwavelength detector and the $\mathrm{I} / \mathrm{O}$ and diagnostic firmware for the HP 1050 liquid chromatograph system. He is currently an $R \& D$ project . earned an engineering diploma in electronics from the Technical University of Karlsruhe in 1985, joining HP shortly after graduation. He was born in Pforzheim, West Germany, and resides in Pfinztal with his wife and two children. Volker's hobbies include photography, an aquarium, motorbiking, meditation, and yoga.

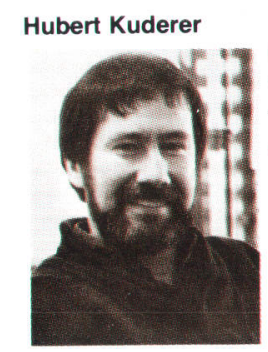

Hubert Kuderer developed the multiwavelength detector for the HP 1050 liquid chromatograph system. He has also designed frontend electronics for the HP 1040A detector, and worked in quality assurance and manufacturing engineering. After receiving an engineering diploma from the Fachhochschule Offenburg, West Germany, in 1978, he joined HP. He has authored technical journal articles on spectograph sensitivity, photodiode array spectrometers, and photodiode architecture, and he is named an inventor in two patents in PDA read- out technology. Born in Offenburg, Hubert resides in Waldbronn with his wife and two children. He enjoys tennis, skiing, and woodworking.

44 - LC Firmware Development =

\section{Christian Büttner}

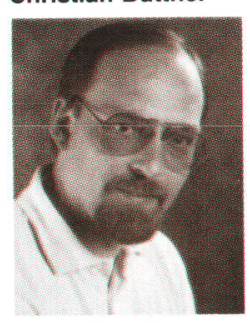

Soon after he received his engineering diploma in electronics (1981) from Fachhochschule Esslingen, Christian Büttner joined HP's Waldbronn Analytical Division in 1981. He designed and implemented firmware for the HP 1050 liquid chromatograph system. He has also designed firmware for the HP 1090 local user interface, and for the HP 1040 detector. As a project manager, he currently is working on HP 1050 communications and firmware enhancements. Christian was born in Stuttgart, West Germany, and now lives with his wife and two children in Waldbronn. He enjoys table tennis, bicycling, and amateur theater.

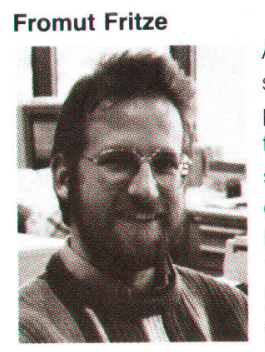

After studies at the University of Karlsruhe in computer science, and graduation from the Fachhochschule Karlsruhe (1982) in electronic engineering Fromut Fritze joined HP in 1982. He designed operating system software and tools for the HP 1050 liquid chromatograph system. Before that, he designed hardware and software for the HP 1090 liquid chromatograph system, a remote control standard, and Pascal ChemStation software. Now a project leader, he is working on a revision control system and software quality. His professional interests center around operating systems and objectoriented techniques. Born in Heidelberg, West Germany, he and his wife live in Karlsruhe. His hobbies include photography, traveling, and desktop publishing

\section{Gerhard Ple}

Author's biography appears elsewhere in this section

51 - HP OpenView Network Management =

Anthony S. Ridolfo

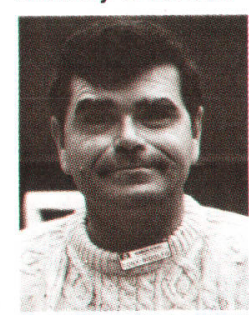

Born in Great Falls, Montana, Tony Ridolfo received his mathematics education at Wabash College (BA degree in 1966), Ohio University (MS in 1968), and lowa State University $(\mathrm{PhD}$ in 1975). After teaching mathematics and computer science as a profes- sor at Murray State University in Kentucky, he joined HP in 1976 and developed firmware for a number of HP's calculators. He also served as a project manager for the HP 75C computer's operating system. Tony coauthored an HP Journal article on the HP 75C in 1983. As a project manager on the HP OpenView project, he started development of HP OpenView Windows and later led the development of the OpenView NS performance monitor. He is a member of the Society for Industrial and Applied Mathematics, and the American Mathematical Society. Tony lives in Saratoga, California, with his wife and three teenage children, is active in the Boy Scouts and as a referee in youth soccer. His leisure activities include golf, skiing, and bridge

\section{$54=$ HP OpenView Architecture}

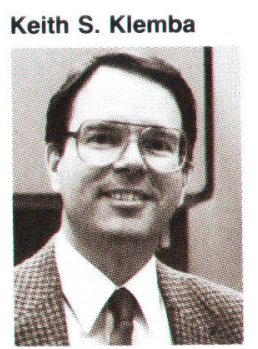

Information networks are the primary professional interest of Keith Klemba, a member of the Information Networks Group technical staff who helped develop the HP OpenView network management architecture. His other professional interests include broadcast and space information networks. He joined HP's Network Architecture Lab in 1986. Before joining $\mathrm{HP}$, he was a product manager with Vitalink Communications Corporation in Fremont, California, and a systems engineer and technical director with SRI International in Menlo Park, California. He received an AA degree (1970) in computer science from DeAnza College. Keith authored a technical paper on HP OpenView architecture, published in the 1989 proceedings of the IFIP. In 1967, he served a tour of duty in Vietnam with the U.S. Army. $\mathrm{He}$ is a member of the IEEE, and a commissioner for the police athletic league's girls' softball for the city of Santa Clara. Married and the father of two children, Keith was born in Chicago, Illinois, and now lives in Santa Clara, California. His hobbies include raising $4-\mathrm{H}$ guide dogs for the blind

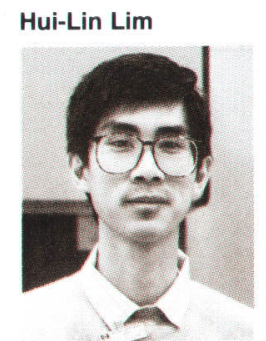

As a member of the technical staff in the Network Architecture Laboratory in HP's Information Network Group, Hui-Lin Lim helped to develop and refine the HP OpenView network management architecture. He joined HP's Singapore Network Operations group in 1988. Before that, he developed office systems and wrote programs for the National Computer Board in Singapore. His professional interests include the UNIX operating system and personal computers. Hui-Lin earned a BSc degree (1983) in computer science from Queen Mary College at the University of London in the United Kingdom. Born in the Republic of Singapore, he lives in Santa Clara, California, with his wife and child. He enjoys photography and science fiction. 


\section{Maureen C. Mellon}

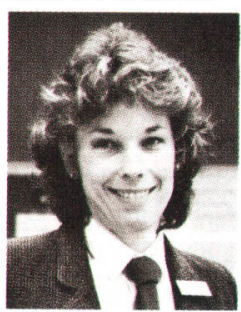

Maureen Mellon is the project manager of a group responsible for developing HP OpenView network management architecture. Maureen joined HP in 1989 and managed four network architecture specialists on this project. She received a BSEE degree (1975) from Villanova University in Villanova, Pennsylvania, an MSEE degree (1976) from Drexel University in Philadelphia, Pennsylvania, and performed additional graduate work towards a PhD (1989) at the University of California at Los Angeles. Before joining HP, Maureen worked on ISDN and broadband ISDN for AT\&T Bell Laboratories in Holmdel, New Jersey. An editor of CCITT recommendation Q.714 on signaling system No. 7, and coauthor of a book on economic characterizations of large telephony networks, Maureen is a member of the IEEE, and Eta Kappa Nu and Tau Beta Pi societies. She was born in Darby, Pennsylvania, and lives in Cupertino, California, with her husband. She enjoys skiing, tennis, running, and hiking.

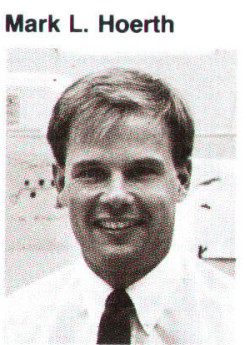

Mark Hoerth is the product manager for the HP OpenView network manager server on the HP-UX operating system. After joining HP in 1987, he served as the product manager for instrument support at HP's Product Support Division. He received a $B S$ degree (1985) in electrical engineering from the University of Nebraska, and an MBA degree (1987) from Stanford University. Mark was born in Rapid City, South Dakota, and now resides with his wife in Stanford, California. His hobbies include photography, swimming, racquetball, and running.

60 - HP OpenView Windows

\section{Arthur J. Kulakow}

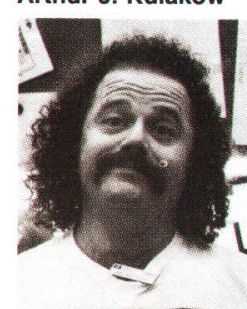

Arthur Kulakow developed the graphics library and the map routines used in HP OpenView Windows. He is now researching methods that will enable software develpers to use $\mathrm{C}++$ in the development of network management platforms. Before joining HP in 1985, Art was a software engineer with Bell Laboratories in Naperville, Illinois, and at Digital Research Company in Monterey, California. He earned a BS degree (1980) and MS degree (1982) in computer science from the University of Wiscon$\sin$ in Milwaukee. He is a member of ACM and his professional interests include user interface design, object-oriented programming, and computer graphics. Born in Milwaukee, Wisconsin, Art lives in San Jose, California. He enjoys scuba diving, hiking, camping, and aerobics.

\section{Kathleen L. Gannon}

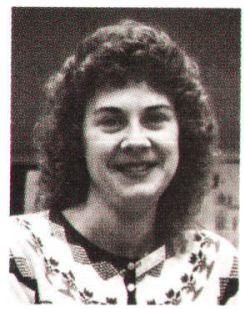

Kathleen Gannon worked as an R\&D software engineer in the development of the HP OpenView Windows/MS-DOS system. Before that, Kathy was a systems engineer at HP Labs. She began her HP career as a product marketing engineer for cooperative support products in 1980. She earned a BS degree (1980) in industrial engineering from Northwestern University in Evanston, Illinois, and an MS degree (1985) in electrical engineering from Stanford Un versity. Born in Pittsburgh, Pennsylvania, Kathy is married and lives in Santa Clara, California. She has been an active member of the board of directors of the Santa Clara Valley Science and Engineering Fair for the past five years. Her hobbies and interests include needlework and science fiction.

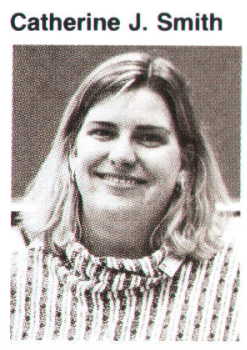

Catherine Smith is the R\&D project manager for the HP OpenView Windows/MS DOS product. Prior to that she served as project manager for the HPMPE XL terminal subsystem. Catherine joined HP in 1978, soon after she graduated from the University of California at Berkeley with a BS degree in electrical engineering and computer science. Born in Yakima, Washington, she resides in San Jose, California. Catherine is married and has one son in college. Her hobbies include downhill skiing and hiking.

66 - HP OpenView BridgeManager

Tamra I. Perez

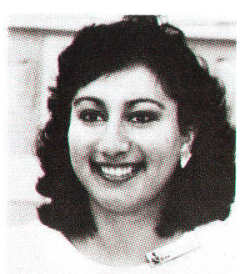

Developing firmware and network software are Tamra Perez' major professional interests. She developed the network inter face for the HP Bridgemanager project, and is now designing a user interface to manage HP's new highspeed and remote bridges After joining HP in 1986 in the Roseville Networks Division, she codeveloped a wire test tool for HP StarLAN-1 products. Before that, Tamra worked in IBM's disk technology division as a summer intern for two years. She earned her BS degree (1986) in computer engineering from San Jose State University, and will receive her MS degree in computer engineering from UC Davis in June 1990. Tamra was born in San Jose, California, and lives in Roseville. She is a member of the Sweet Adelines women's singing quartet, a flute player, and a member of the Tau Beta Pi Engineering Honor Society

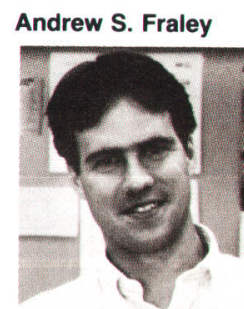

After designing and implementing LAN hubs, PC LAN cards, and drivers, Andrew Fraley worked on the design and development of the HP BridgeManager's user interface. Andy joined HP's Roseville Networks Division as a design engineer in 1986, soon after earning

a BS degree (1986) in computer science from the Massachusetts Institute of Technology. Born in Columbus, Ohio, he and his wife reside in Citrus Heights, California. Andy enjoys soccer, skiing, tennis, and guitar.

\section{1- HP OpenView Data Line Monitor}

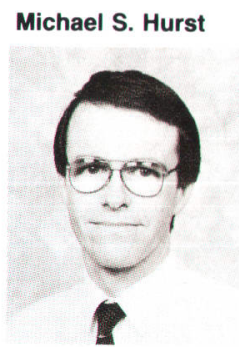

Michael Hurst joined HP in the Queensferry Telecom Division in Scotland shortly after he received his Bachelor of Science degree with honors (1978) in computer science from Edinburgh University in Scotland. Mike was the project manager for the HP OpenView data line monitor software. In the past. he designed microprocessor hardware and firmware for the HP 3779A/B and HP 3776A/B primary multiplex test sets, and HP 1000 application software for the HP 37100 S remote access and test system. He is currently directing new developments for the HP 37100 S system. Mike is a member of the ACM and the British Computer Society where he serves on the Edinburgh Branch Committee. Born in Wiltshire, England, he resides in Edinburgh, Scotland, where he enjoys skiing and running. One of Mike's ambitions is to spend two weeks skiing in Colorado.

76 _ HP OpenView DTC Manager,

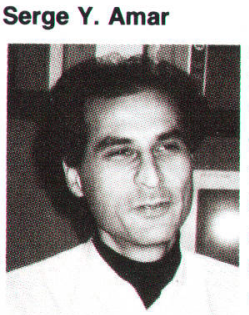

s the technical leader in the development of the HP OpenView Datacommunications and Terminal Controller (DTC) manager, Serge Amar was responsible for its overall design and implementation. He is now a project manager for the OpenView DTC man-

ager. Before that, Serge was a foreign service employee with HP's Information Networks Division in Cupertino, California. He developed the DTC management module for the first release of MPE XL. Prior to joining HP in 1983, he designed electronic credit cards for home banking for Honeywell Bull Co. Serge received his diploma in electronics (1979) from Orsay University south of Paris. Born in Colomb-Bechar, Algeria, he now lives in Grenoble, France, with his wife and two children. His professional interests include personal computers and network management, and his leisure time activities include skiing and diving. 
Michele A. Prieur

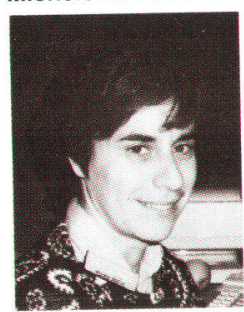

Michele Prieur helped

develop the HP OpenView

Datacommunications and

Terminal Controller (DTC)

manager. Joining HP's

Grenoble Terminal Division

in Grenoble, France, in

1980, she worked as a de-

velopment engineer for the

HP 2392A terminal and as

a technical leader for HP AdvanceLink. She is now a project manager in the development of network management systems. She received an engineering diploma (1980) from the Ecole Supérieure d' Electricité. Born in Lyon, France, Michele and her husband and child now reside in Grenoble. She enjoys horseback riding, reading, and sports
85 - Network Management Application

\section{Lisa M. Cole}

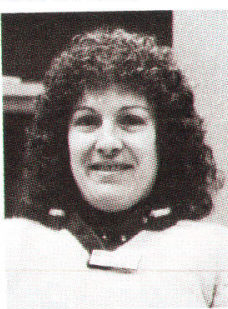

A graduate of Merrimack College in North Andover. Maine, Lisa Cole earned a BS degree (1983) in computer science. After joining $\mathrm{HP}$ in 1987 as a design engineer, she worked on the Vectra portion of the HP OpenView NS Monitor, and $L$ is now developing security software for the communications portion of OpenView. Previously, she worked on network management with Digital Equipment Corporation, on communications applications and SNA with Wang Laboratories, Inc., and on compilers with Honeywell Information Systems. Lisa's professional interests include network management and standards-based communications. A native of Salem, Massachusetts, she lives in Scotts Valley, California. She and her husband are expecting their first child in June. Lisa enjoys skiing, travel, and handicrafts.
Atul R. Garg

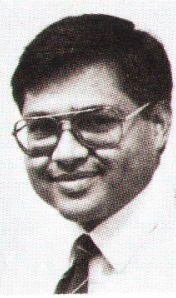

A project manager for the HP OpenView distributed network management services, Atul Garg has worked in the area of network protocols and architecture since he joined HP in 1981. He worked on HP AdvanceNet and on HP's design of the internet architecture and IEEE Standard 802.3. He also contributed to the development of the distributed NS monitor applications under OpenView. Atul coauthored an article on HP AdvanceNet in the HP Journal in October, 1986, and published another article on LAN internet protocols for a Midcon conference in 1982. He earned a bachelor's degree (1979) in electrical engineering from the Indian Institute of Technology in Kanpur, India, and an MS degree (1981) in electrical engineering from the University of Hawaii in Honolulu. Born in New Delhi, India, Atul lives in Santa Clara, California, with his wife and daughter. His hobbies include badminton and bridge. 
Hewlett-Packard Company, 3200 Hillview Avenue, Palo Alto, California 94304

\section{ADDRESS CORRECTION REQUESTED}

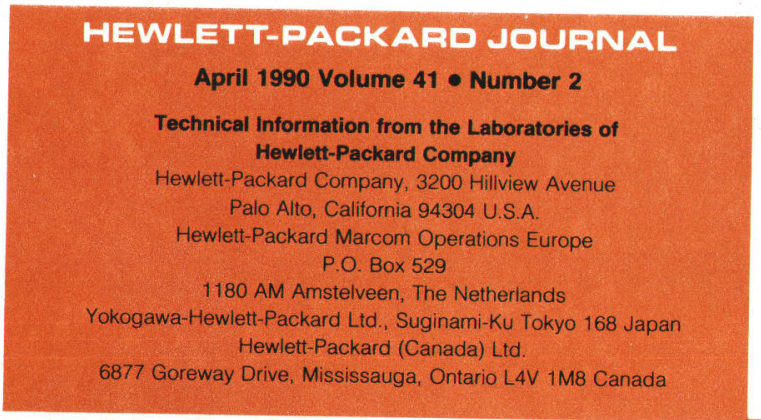

Bulk Rate

U.S. Postage

Paid

Hewlett-Packard Company

CHANGE OF ADDRESS:

00199127

MR. GEORGE PONTIS

SUITE 409

1742 SAND HILL RD

PALO ALTO CA 94304 


\section{HP Archive}

This vintage Hewlett-Packard document was preserved and distributed by www.hparchive.com

Please visit us on the web!

The HP Archive thanks George Pontis for his contribution of this material.

On-line curator: John Miles, KE5FX jmiles@pop.net 
